\title{
Computational Study of the Formation of C8, C5 and C4 Guanine:Lysine Adducts via Oxidation of Guanine by Sulfate Radical Anion
}

\author{
Bishnu Thapa ${ }^{\dagger \text {, Sebastien P. Hebert }}{ }^{\dagger}$, Barbara H. Munk $k^{\dagger \#}$, Cynthia J. Burrows ${ }^{\ddagger}$, \\ H. Bernhard Schlegel ${ }^{*} *$
}

'Department of Chemistry, Wayne State University, Detroit, Michigan 48202

Department of Chemistry, University of Utah, 315 S. 1400 East, Salt Lake City, Utah 84112

§Current address: Department of Chemistry, Indiana University, Bloomington, IN 47405

${ }^{\#}$ Current address: School of Molecular Sciences, Arizona State University, Tempe, AZ 85287

*Corresponding author: Tel: (313) 577-2562. Fax: (313) 577-8822 Email: hbs@chem.wayne.edu

\section{Supporting Information}

\section{C4 addition of methylamine}

C4 lysine:guanine adducts were not isolated in any of the reported experimental studies, indicating that the barriers for key reactions are higher than for $\mathrm{C} 8$ and $\mathrm{C} 5$ addition. Possible pathways for methylamine addition to C4 are shown in Scheme S1 and Figure S1. Water-assisted methylamine addition to guanine radical cation (Gradcat) has a barrier of $24.4 \mathrm{kcal} / \mathrm{mol}$ to form $\mathbf{7 1}$ and is endothermic by $10.5 \mathrm{kcal} / \mathrm{mol}$. Deprotonation of 71 yields two neutral radical tautomers $72\left(\mathrm{p} K_{\mathrm{a}}=7.33\right)$ and $\mathbf{7 3}\left(\mathrm{p} K_{\mathrm{a}}=7.05\right)$ of comparable energy. 72 and 73 can undergo proton-coupled oxidation reactions to form a common zwitterionic intermediate, $76\left(\mathrm{E}^{\mathrm{o}}=0.70 \mathrm{~V}\right.$ and $0.71 \mathrm{~V}$, resp.). It is also possible to form a neutral diradical rather than a zwitterion, but the singlet diradical is higher in energy than the zwitterionic species. Addition of methylamine to the guanine radical (Grad) has a barrier of $30.6 \mathrm{kcal} / \mathrm{mol}$ to form $\mathbf{7 3}$. Addition to the oxidized guanine cation, $\mathbf{G}^{\mathbf{0 x}} \mathbf{c a t}$, is barrierless and forms $74(\Delta \mathrm{H}=-11.1 \mathrm{kcal} / \mathrm{mol})$ which deprotonates $\left(\mathrm{p} K_{\mathrm{a}}=-0.38\right)$ to give the zwitterionic species, 76. 76 also be formed by methylamine addition across C4-N3 
bond of $\mathbf{G}^{\mathbf{0 x}}(8.2 \mathrm{kcal} / \mathrm{mol})$ followed by tautomerization. $\mathbf{7 6}$ can tautomerize and undergo acyl migration to form the 4-methylamine substituted spirocyclic intermediate, 78. In a second nucleophilic addition step, methylamine or water can add across the C8-N7 double bond. The ring rearrangement reaction can also occur after the addition of second nucleophile but the barriers are calculated to be $4-8 \mathrm{kcal} / \mathrm{mol}$ higher. The methylamine addition (barrier height of $17.1 \mathrm{kcal} / \mathrm{mol}$ ) is calculated to be more favored than water addition (barrier height of $21.4 \mathrm{kcal} / \mathrm{mol}$ ). Both the methylamine adduct (79) and the water adduct (83) can undergo subsequent two proton-coupled oxidation reactions to produce the final products, 4,8-diNR-Sp (82) and 4-NR,8-oxo-Sp (87), respectively.

Scheme S1. Possible pathways for the formation of guanine:methylamine adducts via sequential one-electron oxidations of guanine and nucleophilic attack of methylamine $\left(\mathrm{R}=\mathrm{CH}_{3}\right)$ at $\mathrm{C} 4$. Numbers next to the arrow correspond to $\mathrm{p} K_{\mathrm{a}}$ 's (pink, italics) and standard redox potentials $\left(\mathrm{E}^{\circ}\right)$ (blue, regular). The structures shown in red represent the thermodynamically favored pathway. 


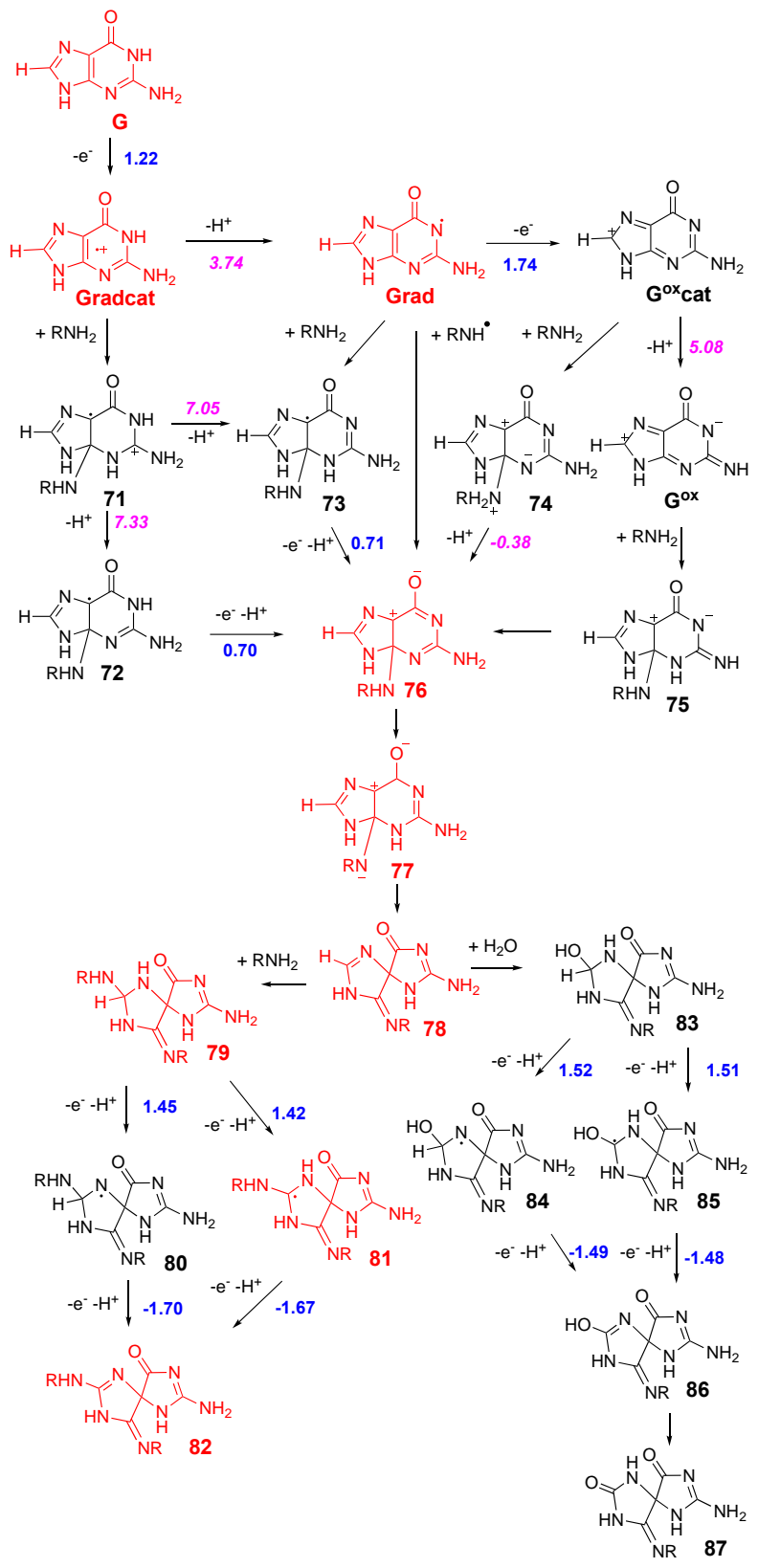




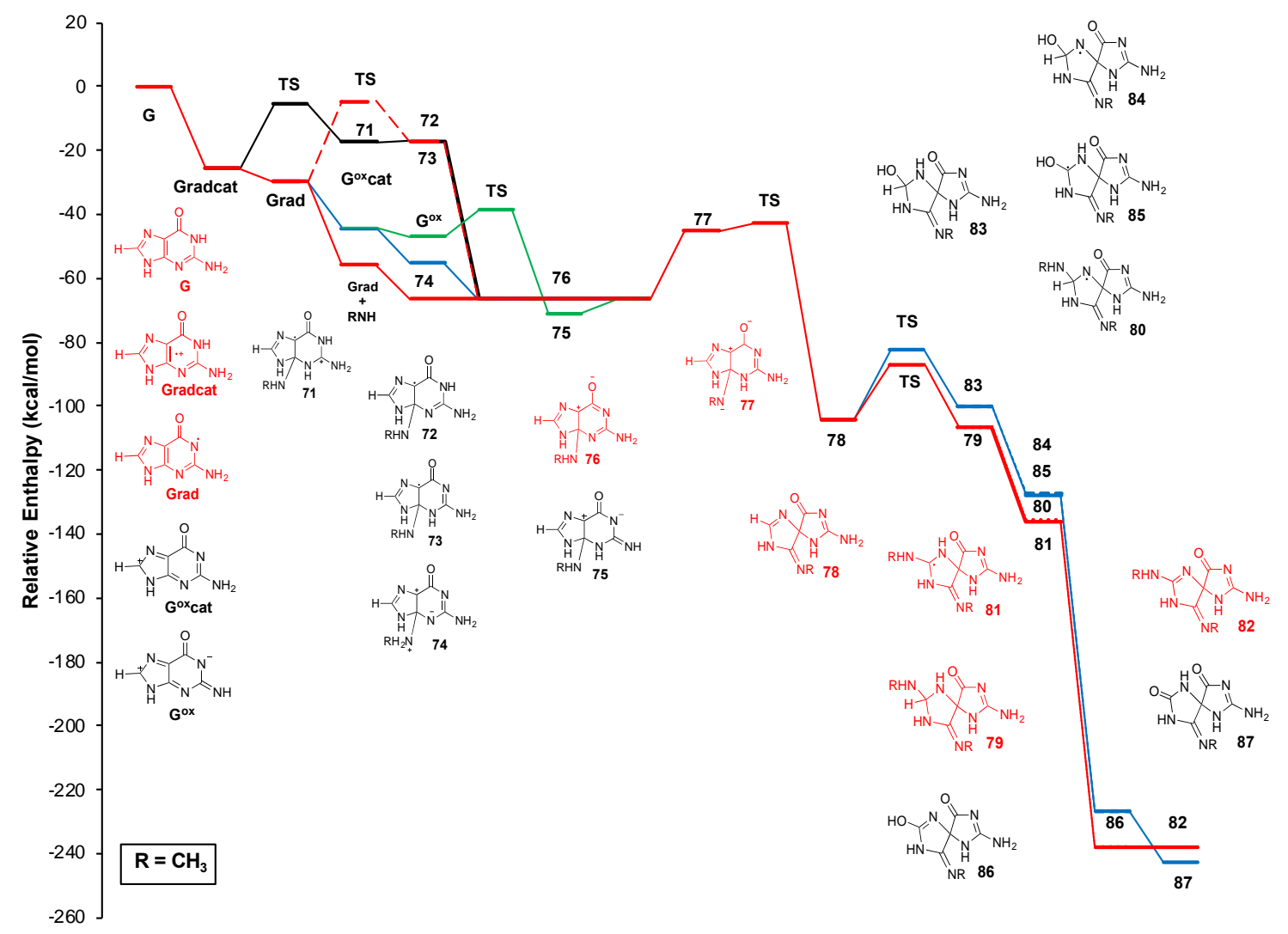

Figure S1. Comparison of the relative enthalpies $(\mathrm{kcal} / \mathrm{mol})$ of adducts resulting from the addition of methylamine at the $\mathrm{C} 4$ position of guanine radical cation (Gradcat, red), guanine radical (Grad, black), oxidized guanine cation ( $\mathbf{G}^{\mathbf{o x}} \mathbf{c a t}$, green) and neutral oxidized guanine ( $\mathbf{G}^{\mathbf{o x}}$, blue) calculated at the SMD/B3LYP/aug-cc-pVTZ//SMD/B3LYP/6-31+G(d,p) level of theory. On the right side of the figure, the red line corresponds to the addition of a second methylamine and the blue line corresponds to the addition of water. The solid red line represents the most favored pathway.

\section{C4 addition of water}

Scheme S2 shows the various possible pathways for water addition to an oxidized guanine intermediate at the $\mathrm{C} 4$ position and Figure $\mathbf{S 2}$ summarizes the thermodynamics. Water addition to $\mathrm{C} 4$ follows almost the same pattern as for methylamine addition. In comparison to the water addition to the guanine radical cation (Gradcat), guanine radical (Grad), and oxidized guanine neutral ( $\mathbf{G}^{\mathbf{o x}}$ ) (barriers of 33.4, 30.1, and $33.7 \mathrm{kcal} / \mathrm{mol}$, resp.), addition of water to the oxidized guanine cation $\left(\mathbf{G}^{\mathbf{0 x}} \mathbf{c a t}\right)$ has smaller barriers (8.5 $\mathrm{kcal} / \mathrm{mol})$. Loss of a proton from $88\left(\mathrm{pK}_{\mathrm{a}}=4.10\right.$ for $\mathrm{N} 1$ and $\mathrm{pK}_{\mathrm{a}}=5.53$ for $\left.\mathrm{N} 3\right)$ produces neutral radicals, 89 and $\mathbf{9 0}$, respectively. Proton-coupled oxidation of $\mathbf{8 9}\left(\mathrm{E}^{\mathrm{o}}=0.86, \mathrm{PCET}\right)$ 
or $90\left(\mathrm{E}^{\mathrm{o}}=0.76\right.$, PCET $)$ forms a zwitterionic species, 92.92 can then tautomerize to form 93. 93 can also be formed by deprotonation of $91\left(\mathrm{p} K_{\mathrm{a}}=2.33\right)$ or tautomerization of 93'. 93 can undergo acyl migration to form the spirocyclic intermediate 94. Addition of methylamine across the C8-N7 double bond of 94 has a barrier of $13.8 \mathrm{kcal} / \mathrm{mol}$ while water addition has a barrier of $24.0 \mathrm{kcal} / \mathrm{mol}$ and forms 95 . Two sequential proton coupled electron transfer reactions form the final products, 4-oxo,8-NHR-Sp, 98 and 4,8-dioxoSp, 103, respectively.

Scheme S2. Possible pathways for the formation of guanine:water adduct at the $\mathrm{C} 4$ position of guanine via sequential one-electron oxidation of guanine and nucleophilic attack of water at $\mathrm{C} 4$. Numbers next to the arrow correspond to $\mathrm{p} K$ a $s$ (pink, italics) and standard redox potentials $\left(\mathrm{E}^{\circ}\right)$ (blue, regular). The structures shown in red represent the thermodynamically favored pathway.

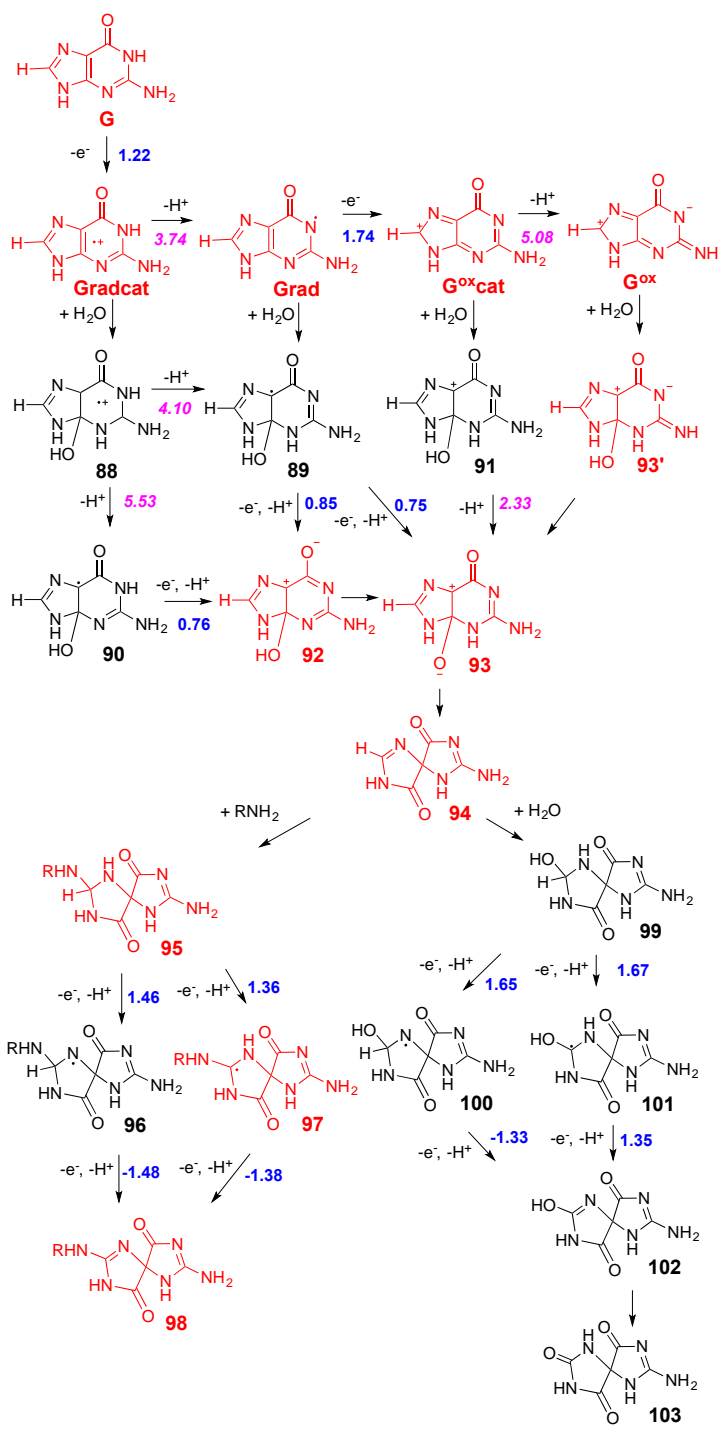




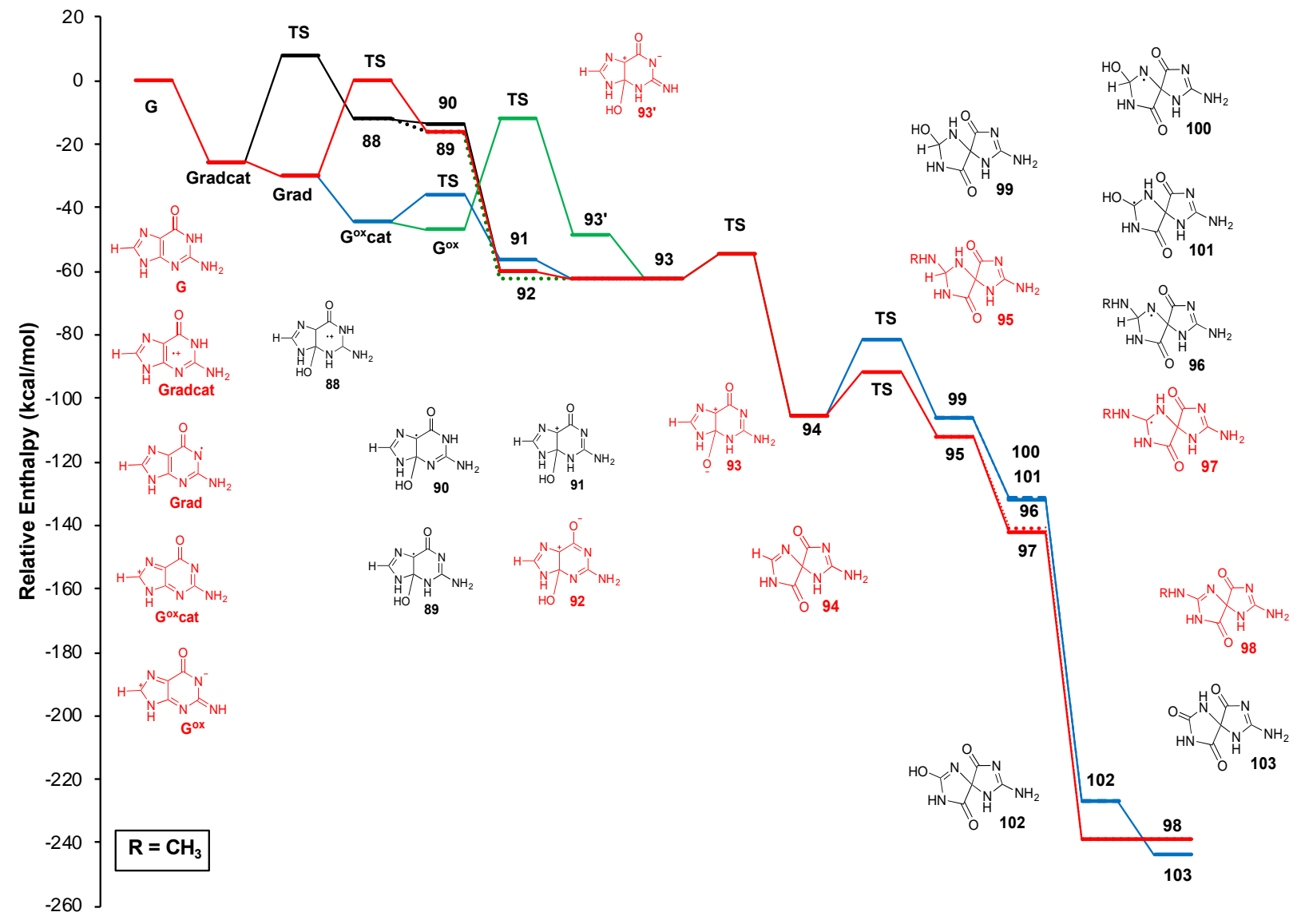

Figure S2. Comparison of the relative enthalpies $(\mathrm{kcal} / \mathrm{mol})$ of adducts resulting from the addition of water at the $\mathrm{C} 4$ position of guanine radical cation (Gradcat, black), guanine radical (Grad, red), oxidized guanine

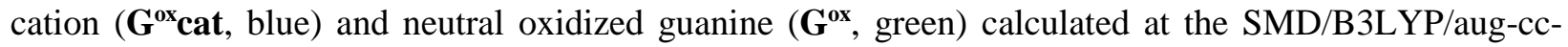
pVTZ//SMD/B3LYP/6-31+G(d,p) level of theory. On the right side of the figure, the red line corresponds to the addition of a second methylamine and the blue line corresponds to the addition of water. 


\section{Geometry Coordinates (SMD/B3LYP/6-31+G(d,p) optimized in water)}

\begin{tabular}{lrrr}
\hline \multicolumn{4}{c}{ Sulfate radical anion } \\
\hline SCF Done: & E(UB3LYP) $=$ & -699.161202188 a.u. \\
-12 & & & \\
S & 0.00000000 & 0.00000000 & 0.07978600 \\
$\mathrm{O}$ & 0.00000000 & 1.24114200 & 0.88832500 \\
$\mathrm{O}$ & 0.00000000 & -1.24114200 & 0.88832500 \\
$\mathrm{O}$ & 1.13082200 & 0.00000000 & -0.96811100 \\
$\mathrm{O}$ & -1.13082200 & 0.00000000 & -0.96811100
\end{tabular}

\begin{tabular}{lrrr}
\hline \multicolumn{4}{c}{ Sulfate dianion (with alpha $=\mathbf{0 . 9 0}$ ) } \\
\hline SCF Done: & $\mathrm{E}(\mathrm{RB} 3 \mathrm{LYP})=$ & -699.394742999 & a.u. \\
-2 & & & \\
$\mathrm{~S}$ & 0.00005700 & -0.00011400 & 0.00020000 \\
$\mathrm{O}$ & 1.31957900 & -0.21302700 & 0.72497400 \\
$\mathrm{O}$ & -1.01414300 & 0.60511200 & 0.95735700 \\
$\mathrm{O}$ & -0.51931300 & -1.33484300 & -0.51010100 \\
$\mathrm{O}$ & 0.21376400 & 0.94298500 & -1.17263000
\end{tabular}

Sulfate radical anion (8 explicit waters)

SCF Done E(RB3LYP $)=:-1310.78071329$ a.u.

$-12$

$\begin{array}{llcc}\mathrm{O} & -1.801529 & 0.208494 & 1.052681 \\ \mathrm{~S} & -0.635967 & -0.221409 & 1.965837 \\ \mathrm{O} & -0.533198 & 0.644366 & 3.163446 \\ \mathrm{O} & 0.630977 & -0.365061 & 1.211925 \\ \mathrm{O} & -1.256163 & -1.592723 & 2.304644 \\ \mathrm{O} & 2.339263 & 1.973824 & 0.687084 \\ \mathrm{H} & 3.246763 & 1.786986 & 0.969781 \\ \mathrm{H} & 1.858002 & 1.135905 & 0.818063 \\ \mathrm{O} & 0.943033 & 3.289868 & 2.778208 \\ \mathrm{H} & 0.378302 & 2.544873 & 3.042002 \\ \mathrm{H} & 1.483489 & 2.919379 & 2.047669 \\ \mathrm{O} & 1.775548 & 0.510440 & 4.991502 \\ \mathrm{H} & 2.138878 & 1.405436 & 5.065165 \\ \mathrm{H} & 0.970914 & 0.612692 & 4.450634 \\ \mathrm{O} & 3.133503 & -0.856017 & 2.905540 \\ \mathrm{H} & 2.436281 & -0.784830 & 2.233017 \\ \mathrm{H} & 2.751500 & -0.391526 & 3.680954 \\ \mathrm{O} & -1.749197 & -3.719025 & 0.218590 \\ \mathrm{H} & -2.530547 & -4.219517 & 0.497050 \\ \mathrm{H} & -1.524638 & -3.160041 & 0.982387 \\ \mathrm{O} & -2.458301 & -1.512524 & -1.423219 \\ \mathrm{H} & -2.249289 & -2.331330 & -0.924690 \\ \mathrm{H} & -2.257911 & -0.803037 & -0.792241 \\ \mathrm{O} & -3.995439 & -1.615712 & 3.811031 \\ \mathrm{H} & -3.086520 & -1.795875 & 3.524419 \\ \mathrm{H} & -4.249650 & -0.820594 & 3.294666 \\ \mathrm{O} & -4.514973 & 0.694084 & 2.248869 \\ \mathrm{H} & -3.668401 & 0.664287 & 1.770115 \\ & & & \\ & & & \end{array}$

$\mathrm{H} \quad-5.189372 \quad 0.517872 \quad 1.576247$

Sulfate dianion (8 explicit waters)

SCF Done E(RB3LYP) =: $\quad-1311.02927194$ a.u. $-21$

$\begin{array}{llll}\mathrm{O} & -1.649051 & 0.413316 & 1.110619\end{array}$

S $\quad-0.593771 \quad-0.205536 \quad 2.012943$

$\begin{array}{llll}\mathrm{O} & -0.421384 & 0.667389 & 3.245231\end{array}$

$\begin{array}{llll}\mathrm{O} & 0.716568 & -0.303692 & 1.248578\end{array}$

$\begin{array}{llll}\mathrm{O} & -1.022496 & -1.597714 & 2.446477\end{array}$

$\begin{array}{llll}\text { O } & 2.089054 & 2.006014 & 0.492878\end{array}$

$\begin{array}{llll}\mathrm{H} & 3.015725 & 1.825915 & 0.708027\end{array}$

$\begin{array}{llll}\mathrm{H} & 1.607822 & 1.180244 & 0.731729\end{array}$

$\begin{array}{llll}\text { O } & 0.836984 & 3.188043 & 2.777023\end{array}$

$\begin{array}{llll}\mathrm{H} & 0.352065 & 2.364530 & 2.992672\end{array}$

$\mathrm{H} \quad 1.348658 \quad 2.936986 \quad 1.980115$

$\begin{array}{llll}\mathrm{O} & 1.663028 & 0.255028 & 5.049149\end{array}$

$\begin{array}{llll}\mathrm{H} & 2.018630 & 1.140306 & 5.214261\end{array}$

$\begin{array}{llll}\mathrm{H} & 0.907741 & 0.395732 & 4.432628\end{array}$

$\begin{array}{llll}\mathrm{O} & 3.042087 & -0.863685 & 2.809350\end{array}$

$\begin{array}{llll}\mathrm{H} & 2.276697 & -0.724133 & 2.214046\end{array}$

$\begin{array}{llll}\mathrm{H} & 2.721567 & -0.506868 & 3.663612\end{array}$

$\begin{array}{llll}\mathrm{O} & -1.952651 & -3.466880 & 0.594845\end{array}$

$\begin{array}{llll}\mathrm{H} & -2.771348 & -3.808635 & 0.982523\end{array}$

$\mathrm{H} \quad-1.610326-2.821136 \quad 1.255135$

$\begin{array}{llll}\text { O } & -2.548178 & -1.212414 & -1.054764\end{array}$

$\begin{array}{llll}\mathrm{H} & -2.421335 & -2.084244 & -0.626324\end{array}$

$\mathrm{H} \quad-2.231406-0.586852 \quad-0.370411$

$\begin{array}{llll}\mathrm{O} & -3.663830 & -1.763154 & 3.516721\end{array}$

$\mathrm{H} \quad-2.730522 \quad-1.753432 \quad 3.218742$

$\mathrm{H} \quad-4.063194 \quad-1.029138 \quad 3.005821$

$\begin{array}{llll}\mathrm{O} & -4.337965 & 0.462489 & 1.847419\end{array}$

$\begin{array}{llll}\mathrm{H} & -3.387322 & 0.469391 & 1.589067\end{array}$

$\mathrm{H} \quad \begin{array}{llll}-4.806490 & 0.141416 & 1.063267\end{array}$
$\mathrm{O}_{2}$ (4 explicit waters)

SCF Done: $E($ RB3LYP $)=-456.131708532$ A.U.

03

$\begin{array}{llll}\mathrm{O} & -0.800942 & 0.511823 & -0.232404 \\ \mathrm{O} & -0.019553 & 0.738064 & -1.133793 \\ \mathrm{O} & -3.036074 & 3.150615 & -0.140315\end{array}$ 


$\begin{array}{llcc}\mathrm{H} & -2.603309 & 3.171909 & -1.021873 \\ \mathrm{H} & -2.646443 & 2.385728 & 0.306536 \\ \mathrm{O} & 0.599209 & -2.550854 & 0.627944 \\ \mathrm{H} & 1.092299 & -2.572100 & 1.461709 \\ \mathrm{H} & -0.032976 & -1.823561 & 0.732686 \\ \mathrm{O} & -1.799598 & 3.280671 & -2.664565 \\ \mathrm{H} & -1.172661 & 2.541929 & -2.692656 \\ \mathrm{H} & -2.453886 & 3.075691 & -3.349177 \\ \mathrm{O} & 2.284542 & -1.775671 & -1.487741 \\ \mathrm{H} & 2.001726 & -0.879633 & -1.719717 \\ \mathrm{H} & 1.703575 & -2.033224 & -0.738652\end{array}$

$\mathrm{O}_{2}{ }^{*}$ (4 explicit waters)

SCF Done: $\mathrm{E}(\mathrm{RB} 3 \mathrm{LYP})=-456.298582075$ A.U.

$-12$

$\begin{array}{llcc}\mathrm{O} & -0.805643 & 0.329723 & -0.300646 \\ \mathrm{O} & -0.177825 & 0.701872 & -1.421092 \\ \mathrm{O} & -2.725468 & 2.335004 & 0.004265 \\ \mathrm{H} & -2.475214 & 2.808953 & -0.811174 \\ \mathrm{H} & -2.095177 & 1.576813 & 0.002397 \\ \mathrm{O} & 0.320175 & -1.982434 & 0.712658 \\ \mathrm{H} & 0.817819 & -1.739065 & 1.506427 \\ \mathrm{H} & -0.091769 & -1.139570 & 0.394147 \\ \mathrm{O} & -1.303578 & 3.014126 & -2.434287 \\ \mathrm{H} & -0.891623 & 2.171259 & -2.115798 \\ \mathrm{H} & -1.801144 & 2.770783 & -3.228113 \\ \mathrm{O} & 1.741947 & -1.303471 & -1.726047 \\ \mathrm{H} & 1.111698 & -0.545247 & -1.724189 \\ \mathrm{H} & 1.491709 & -1.777358 & -0.910567\end{array}$

$\frac{\mathrm{O}_{2}{ }^{2-} \text { (4 explicit waters) }}{\overline{S C F} \text { Done: }}$

SCF Done: $\mathrm{E}(\mathrm{RB} 3 \mathrm{LYP})=-456.434814942$ A.U. $-21$

$\begin{array}{llcc}\mathrm{O} & -1.418453 & -0.351725 & -0.869754 \\ \mathrm{O} & -0.659094 & 0.191273 & -2.050972 \\ \mathrm{O} & -2.209312 & 1.838446 & 0.259408 \\ \mathrm{H} & -1.856836 & 2.471542 & -0.392387 \\ \mathrm{H} & -1.916656 & 0.942828 & -0.149986 \\ \mathrm{O} & 0.191514 & -1.344933 & 0.819282 \\ \mathrm{H} & 0.408604 & -0.643579 & 1.448935 \\ \mathrm{H} & -0.455971 & -0.909063 & 0.117465 \\ \mathrm{O} & -0.791109 & 2.717683 & -2.125098 \\ \mathrm{H} & -0.762654 & 1.667658 & -2.090397 \\ \mathrm{H} & -1.418477 & 2.942947 & -2.825798 \\ \mathrm{O} & 1.689156 & -0.794467 & -1.628217 \\ \mathrm{H} & 0.771306 & -0.383557 & -1.841616 \\ \mathrm{H} & 1.543892 & -1.123665 & -0.722885\end{array}$

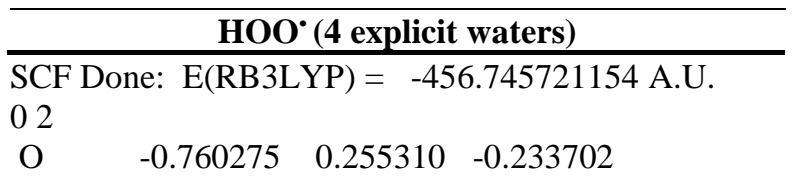

$\begin{array}{llll}\mathrm{O} & -0.133795 & 0.635651 & -1.340746 \\ \mathrm{O} & -2.756459 & 2.415186 & -0.130497 \\ \mathrm{H} & -2.435192 & 2.813309 & -0.963867 \\ \mathrm{H} & -2.168827 & 1.644709 & -0.018904 \\ \mathrm{O} & 0.545371 & -2.246650 & 0.585776 \\ \mathrm{H} & 1.074554 & -2.180983 & 1.394363 \\ \mathrm{H} & 0.021189 & -1.423082 & 0.549719 \\ \mathrm{O} & -1.356855 & 3.100386 & -2.550079 \\ \mathrm{H} & -0.870428 & 2.280476 & -2.353810 \\ \mathrm{H} & -1.867313 & 2.904541 & -3.349576 \\ \mathrm{O} & 1.694644 & -1.106096 & -1.615071 \\ \mathrm{H} & 0.621528 & -0.070183 & -1.510253 \\ \mathrm{H} & 1.442140 & -1.710627 & -0.874790 \\ \mathrm{H} & 1.605538 & -1.619143 & -2.432908\end{array}$

HOO- $^{-}$(4 explicit waters)

\begin{tabular}{|c|c|c|c|}
\hline \multicolumn{4}{|c|}{$\begin{array}{l}\text { SCF Done: } \mathrm{E}(\mathrm{RB} 3 \mathrm{LYP})=-456.92300268 \\
-11\end{array}$} \\
\hline $\mathrm{O}$ & -0.255588 & 0.834337 & 0.369962 \\
\hline $\mathrm{O}$ & 0.512118 & 1.307008 & -0.811410 \\
\hline $\mathrm{O}$ & -2.716918 & 1.141159 & -0.549784 \\
\hline $\mathrm{H}$ & -2.544801 & 1.754378 & -1.285147 \\
\hline $\mathrm{H}$ & -1.795432 & 0.995832 & -0.164739 \\
\hline $\mathrm{O}$ & 0.202114 & -1.707127 & 0.555332 \\
\hline $\mathrm{H}$ & 0.954538 & -1.852065 & 1.146391 \\
\hline $\mathrm{H}$ & 0.043227 & -0.696510 & 0.540905 \\
\hline $\mathrm{O}$ & -1.159572 & 2.862428 & -2.387208 \\
\hline $\mathrm{H}$ & -0.529612 & 2.304850 & -1.868340 \\
\hline $\mathrm{H}$ & -1.081602 & 2.551415 & -3.300218 \\
\hline $\mathrm{O}$ & 1.100395 & -1.212429 & -1.995348 \\
\hline $\mathrm{H}$ & 0.6989 & 0.474544 & -1.304 \\
\hline $\mathrm{H}$ & 0.787680 & -1.590226 & -1.137535 \\
\hline $\mathrm{H}$ & 0.440372 & -1.474790 & -2.653075 \\
\hline
\end{tabular}

HOOH (4 explicit waters)

\begin{tabular}{llcc}
\hline \multicolumn{4}{l}{ SCF Done: $\mathrm{E}(\mathrm{RB} 3 \mathrm{LYP})=-457.395079057$} \\
$\mathrm{O} 1$ & & & \\
$\mathrm{O}$ & 0.000176 & 0.942397 & 0.283690 \\
$\mathrm{O}$ & 0.719363 & 1.254314 & -0.944613 \\
$\mathrm{O}$ & -2.532773 & 1.540726 & -0.479118 \\
$\mathrm{H}$ & -2.239227 & 2.099323 & -1.235362 \\
$\mathrm{H}$ & -0.947198 & 1.143141 & 0.036168 \\
$\mathrm{O}$ & 0.209832 & -1.867392 & 0.593162 \\
$\mathrm{H}$ & 0.938840 & -2.104616 & 1.184759 \\
$\mathrm{H}$ & 0.138578 & -0.889663 & 0.643753 \\
$\mathrm{O}$ & -1.097789 & 2.833020 & -2.462557 \\
$\mathrm{H}$ & -0.371058 & 2.302572 & -2.070737 \\
$\mathrm{H}$ & -1.242450 & 2.461558 & -3.345126 \\
$\mathrm{O}$ & 1.087309 & -1.235443 & -1.960636 \\
$\mathrm{H}$ & 0.833793 & 0.353893 & -1.362308 \\
$\mathrm{H}$ & 0.784027 & -1.685084 & -1.138681 \\
$\mathrm{H}$ & 0.436766 & -1.463197 & -2.641051 \\
$\mathrm{H}$ & -2.945124 & 2.148925 & 0.151977
\end{tabular}




\begin{tabular}{lrrr}
\hline \multicolumn{4}{c}{ Imidazole } \\
\hline SCF Done: & E(RB3LYP) $=$ & -226.250742195 A.U. \\
$0 ~ 1$ & & & \\
$\mathrm{C}$ & 0.58186900 & 1.00111700 & 0.00000100 \\
$\mathrm{C}$ & 1.15110400 & -0.24822900 & 0.00000000 \\
$\mathrm{C}$ & -0.97795900 & -0.56347700 & 0.00000000 \\
$\mathrm{~N}$ & -0.77915100 & 0.78086700 & 0.00000000 \\
$\mathrm{H}$ & -1.50247800 & 1.48966800 & 0.00000600 \\
$\mathrm{H}$ & 1.00186600 & 1.99529800 & -0.00000900 \\
$\mathrm{H}$ & 2.20203000 & -0.49963600 & 0.00000000 \\
$\mathrm{H}$ & -1.96525800 & -1.00151700 & -0.00000400 \\
$\mathrm{~N}$ & 0.16968700 & -1.22662100 & 0.00000000
\end{tabular}

Imidazole Cation

SCF Done: $\mathrm{E}(\mathrm{RB} 3 \mathrm{LYP})=-226.708382892$ A.U.

11

$\begin{array}{lrcc}\mathrm{C} & -0.68136700 & 0.97742000 & 0.00000000 \\ \mathrm{C} & 0.68185200 & 0.97705300 & 0.00000000 \\ \mathrm{C} & -0.00029300 & -1.13937400 & -0.00000100 \\ \mathrm{~N} & -1.07515700 & -0.34813100 & 0.00000100 \\ \mathrm{H} & -2.03354000 & -0.68315400 & -0.00000200 \\ \mathrm{H} & -1.39361200 & 1.78661300 & 0.00000100 \\ \mathrm{H} & 1.39460800 & 1.78579700 & -0.00000200 \\ \mathrm{H} & -0.00065500 & -2.21803800 & 0.00000100 \\ \mathrm{~N} & 1.07498500 & -0.34868800 & 0.00000100 \\ \mathrm{H} & 2.03325000 & -0.68407200 & 0.00000000\end{array}$

\begin{tabular}{lrrr}
\hline \multicolumn{4}{c}{ Methylamine } \\
\hline SCF Done: & E(RB3LYP) $=$ & -95.8787924904 A.U. \\
$0 ~ 1$ & & & \\
C & 0.05417800 & 0.70777000 & 0.00000000 \\
H & 0.58702800 & 1.07242200 & 0.88249200 \\
H & 0.58702800 & 1.07242200 & -0.88249200 \\
H & -0.94856400 & 1.15759500 & 0.00000000 \\
N & 0.05417800 & -0.76525800 & 0.00000000 \\
H & -0.46490200 & -1.09612300 & 0.81122000 \\
H & -0.46490200 & -1.09612300 & -0.81122000
\end{tabular}

\begin{tabular}{llcc}
$\mathrm{H}$ & -0.65567000 & 1.05145500 & -0.15873500 \\
$\mathrm{H}$ & -0.92441100 & -0.07781500 & -1.48148000 \\
$\mathrm{C}$ & -2.19774900 & -0.40329600 & 0.23825600 \\
$\mathrm{H}$ & -2.40346100 & -1.46289100 & 0.02867600 \\
$\mathrm{H}$ & -2.13702500 & -0.30381000 & 1.33149700 \\
$\mathrm{C}$ & -3.37062000 & 0.44008400 & -0.27266500 \\
$\mathrm{H}$ & -3.17287400 & 1.49996500 & -0.07463000 \\
$\mathrm{H}$ & -3.44940600 & 0.33479100 & -1.36089000 \\
$\mathrm{~N}$ & -4.68394000 & 0.11034500 & 0.30900900 \\
$\mathrm{H}$ & -4.62656700 & 0.20561600 & 1.32243700 \\
$\mathrm{H}$ & -4.87943500 & -0.87564100 & 0.13821800 \\
$\mathrm{O}$ & 3.07514500 & 0.74320000 & 0.97359400 \\
$\mathrm{H}$ & 3.25823400 & -0.23020000 & 1.08343200 \\
$\mathrm{H}$ & 2.22406100 & -2.47402100 & 0.0236940 \\
\hline \multicolumn{4}{c}{ Guanine (G) } \\
\hline SCF
\end{tabular}

SCF Done: $\mathrm{E}(\mathrm{RB} 3 \mathrm{LYP})=-542.635312440$ A.U.

01

$\begin{array}{lllll}\text { C } & 0.84037200 & 0.50174100 & -0.00108800\end{array}$

$\begin{array}{llll}\text { C } & 0.53428300 & -0.86081900 & -0.00033800\end{array}$

$\begin{array}{llll}\text { C } & -1.67911500 & -0.57244700 & -0.00152500\end{array}$

$\begin{array}{lllll}\text { C } & & -0.23166300 & 1.43701200 & -0.00001700\end{array}$

$\begin{array}{llll}\mathrm{C} & 2.71457000 & -0.51704900 & 0.00145400\end{array}$

$\begin{array}{llll}\mathrm{H} & -2.29150700 & 1.40185700 & -0.00721000\end{array}$

$\mathrm{H} \quad 3.76579100 \quad-0.76603700 \quad 0.00224900$

$\begin{array}{llll}\mathrm{H} & 1.90601200 & -2.49717200 & 0.00142100\end{array}$

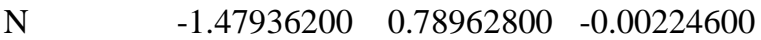

$\begin{array}{llll}\mathrm{N} & -0.68458000 & -1.44658000 & 0.00262400\end{array}$

$\begin{array}{llll}\mathrm{O} & -0.18191800 & 2.68443800 & 0.00305300\end{array}$

$\begin{array}{llll}\mathrm{N} & 2.21470300 & 0.69896200 & 0.00031400\end{array}$

$\mathrm{N} \quad \begin{array}{llll}\mathrm{N} & 1.74649000 & -1.49602000 & 0.00137900\end{array}$

$\mathrm{N} \quad \begin{array}{llll}\mathrm{N} & -2.96350300 & -1.00278200 & -0.05224700\end{array}$

$\mathrm{H} \quad \begin{array}{llll}\mathrm{H} & -3.71104600 & -0.36536300 & 0.19091700\end{array}$

$\mathrm{H} \quad-3.12082700 \quad-1.98187400 \quad 0.14852100$

\section{Guanine Radical Cation (Gradcat)}

\begin{tabular}{lrrr}
\hline SCF Done: & $\mathrm{E}(\mathrm{UB} 3 \mathrm{LYP})=$ & $-542.430288212 \mathrm{~A} . \mathrm{U}$. \\
12 & & & \\
$\mathrm{C}$ & 0.83864300 & 0.51782300 & -0.00000600 \\
$\mathrm{C}$ & 0.54038100 & -0.88447400 & -0.00000600 \\
$\mathrm{C}$ & -1.67640700 & -0.56630300 & 0.00001200 \\
$\mathrm{C}$ & -0.27049400 & 1.45586100 & 0.00000300 \\
$\mathrm{C}$ & 2.69815600 & -0.49842500 & -0.00002100 \\
$\mathrm{H}$ & -2.32930000 & 1.39054000 & 0.00001900 \\
$\mathrm{H}$ & 3.75874000 & -0.70616300 & -0.00002900 \\
$\mathrm{H}$ & 1.96739700 & -2.48744200 & -0.00002000 \\
$\mathrm{~N}$ & -1.50408300 & 0.79116600 & 0.00000900 \\
$\mathrm{~N}$ & -0.64200500 & -1.45462400 & 0.00000200 \\
$\mathrm{O}$ & -0.20390700 & 2.68053300 & 0.00000600 \\
$\mathrm{~N}$ & 2.16140200 & 0.73625100 & -0.00001500 \\
$\mathrm{~N}$ & 1.77377000 & -1.48901700 & -0.00001800 \\
$\mathrm{~N}$ & -2.90253500 & -1.05273100 & 0.00002500 \\
$\mathrm{H}$ & -3.71756600 & -0.44667500 & 0.00003700 \\
$\mathrm{H}$ & -3.03553700 & -2.05873400 & 0.00002800
\end{tabular}




\begin{tabular}{lrrr}
\hline \multicolumn{4}{c}{ Guanine Radical (Grad) } \\
\hline SCF Done: & E(UB3LYP) $=$ & -541.980639737 A.U. \\
02 & & & \\
$\mathrm{C}$ & 0.79835600 & 0.50991400 & -0.00000100 \\
$\mathrm{C}$ & 0.50498900 & -0.88773200 & 0.00000100 \\
$\mathrm{C}$ & -1.69332500 & -0.46944700 & 0.00000300 \\
$\mathrm{C}$ & -0.33195300 & 1.43722100 & 0.00000000 \\
$\mathrm{C}$ & 2.66600200 & -0.50735000 & -0.00000200 \\
$\mathrm{H}$ & 3.72628300 & -0.71649500 & -0.00000200 \\
$\mathrm{H}$ & 1.93570900 & -2.49679500 & -0.00000100 \\
$\mathrm{~N}$ & -1.57072200 & 0.86220800 & 0.00000100 \\
$\mathrm{~N}$ & -0.68447500 & -1.43440500 & 0.00000600 \\
$\mathrm{O}$ & -0.17131700 & 2.67956600 & -0.00000100 \\
$\mathrm{~N}$ & 2.13117100 & 0.72397400 & -0.00000200 \\
$\mathrm{~N}$ & 1.74172900 & -1.50014900 & -0.00000200 \\
$\mathrm{~N}$ & -2.92691000 & -0.98780500 & 0.00001900 \\
$\mathrm{H}$ & -3.73208800 & -0.37500200 & -0.00007500 \\
$\mathrm{H}$ & -3.05933000 & -1.99063800 & -0.00008800
\end{tabular}

8- $\mathrm{NH}_{2} \mathrm{CH}_{3}$ Guanine Radical Cation (1)

SCF Done: E(UB3LYP) $=-638.334177484$ a.u.

12

$\begin{array}{lrcc}\mathrm{C} & 0.13758700 & 0.75444100 & -0.17122900 \\ \mathrm{C} & 0.11714500 & -0.67209500 & -0.20754200 \\ \mathrm{C} & 2.33035400 & -0.88191900 & 0.08789600 \\ \mathrm{C} & 1.39938500 & 1.40019200 & 0.03844100 \\ \mathrm{H} & -1.47990700 & -1.94598000 & -0.70758200 \\ \mathrm{~N} & 2.45468200 & 0.47970600 & 0.16270500 \\ \mathrm{~N} & 1.15170600 & -1.49891200 & -0.08934200 \\ \mathrm{O} & 1.62865600 & 2.62249000 & 0.11052400 \\ \mathrm{~N} & -1.17862000 & -1.03432600 & -0.38234900\end{array}$

\begin{tabular}{lrcc}
\hline \multicolumn{4}{c}{ 8-NHCH3-N7H Guanine Radical Cation (2) } \\
\hline SCF Done: & E(UB3LYP) $=$ & -638.335763215 & a.u. \\
$1 \mathrm{~N}$ & & & \\
$\mathrm{C}$ & -4.21621000 & 0.19731500 & 0.40941500 \\
$\mathrm{H}$ & -4.91867900 & -0.04192800 & 1.20954700 \\
$\mathrm{H}$ & -4.69842400 & -0.01989500 & -0.54735800 \\
$\mathrm{~N}$ & -3.02408700 & -0.65467000 & 0.55678500 \\
$\mathrm{H}$ & -2.59712500 & -0.51546800 & 1.47194000 \\
$\mathrm{C}$ & 0.03945400 & 0.57950700 & -0.20900000 \\
$\mathrm{C}$ & 0.24265500 & -0.83626100 & -0.21975300 \\
$\mathrm{C}$ & 2.45688800 & -0.62510900 & 0.11772000 \\
$\mathrm{C}$ & 1.15411600 & 1.45673200 & -0.02273000 \\
$\mathrm{H}$ & 3.18870500 & 1.30377200 & 0.26630000 \\
$\mathrm{H}$ & -1.11739100 & -2.41366500 & -0.42741900 \\
$\mathrm{~N}$ & 2.35055200 & 0.74351300 & 0.12354400 \\
$\mathrm{~N}$ & 1.41122800 & -1.44915200 & -0.06261000 \\
$\mathrm{O}$ & 1.13046000 & 2.69573400 & 0.01025700 \\
$\mathrm{~N}$ & -0.95213000 & -1.41401500 & -0.40382400 \\
$\mathrm{~N}$ & 3.66673500 & -1.14833500 & 0.30204100 \\
$\mathrm{H}$ & 4.48167000 & -0.56635800 & 0.45213400
\end{tabular}

\begin{tabular}{lrrr}
\hline \multicolumn{4}{c}{ Oxidized Guanine Cation (Gox $\mathbf{c a t})$} \\
\hline SCF Done: & E(RB3LYP) & -541.758118396 A.U. \\
11 & & & \\
$\mathrm{C}$ & 0.79677300 & 0.54523000 & 0.00037100 \\
$\mathrm{C}$ & 0.51637100 & -0.89742100 & 0.00035500 \\
$\mathrm{C}$ & -1.69417300 & -0.47385000 & -0.00011500 \\
$\mathrm{C}$ & -0.38193000 & 1.46021300 & -0.00000100 \\
$\mathrm{C}$ & 2.65908300 & -0.49607800 & 0.00002000 \\
$\mathrm{H}$ & 3.73045900 & -0.65315800 & 0.00018200 \\
$\mathrm{H}$ & 2.01679400 & -2.48129000 & 0.00012700 \\
$\mathrm{~N}$ & -1.59705200 & 0.84611100 & -0.00008100 \\
$\mathrm{~N}$ & -0.62981000 & -1.44908000 & -0.00051500 \\
$\mathrm{O}$ & -0.22687900 & 2.68464900 & -0.00008800 \\
$\mathrm{~N}$ & 2.07515900 & 0.77748400 & -0.00013500 \\
$\mathrm{~N}$ & 1.79318700 & -1.48427800 & -0.00003300 \\
$\mathrm{~N}$ & -2.87541400 & -1.06143400 & 0.00018900 \\
$\mathrm{H}$ & -3.72178900 & -0.49946600 & 0.00072700 \\
$\mathrm{H}$ & -2.94965400 & -2.07346500 & -0.00008500
\end{tabular}

$\begin{array}{lrrr}\mathrm{N} & 3.44022600 & -1.61797500 & 0.20169200 \\ \mathrm{H} & 4.34905400 & -1.19147000 & 0.32573500 \\ \mathrm{H} & 3.36968600 & -2.62487300 & 0.14271200 \\ \mathrm{C} & -1.96416300 & 0.17324700 & -0.53948500 \\ \mathrm{H} & -2.49773800 & 0.20323600 & -1.49407500 \\ \mathrm{~N} & -1.07674600 & 1.29851900 & -0.36271900 \\ \mathrm{H} & -3.44221000 & 1.19367000 & 0.48558800 \\ \mathrm{H} & 3.37826700 & 0.88434800 & 0.29878100 \\ \mathrm{~N} & -3.05886400 & 0.24191300 & 0.52098600 \\ \mathrm{C} & -4.17198800 & -0.74219800 & 0.34692500 \\ \mathrm{H} & -3.77293700 & -1.75206800 & 0.43111600 \\ \mathrm{H} & -4.90242600 & -0.56198700 & 1.13491700 \\ \mathrm{H} & -4.62214900 & -0.58413800 & -0.63287700 \\ \mathrm{H} & -2.62549400 & 0.12685400 & 1.44464600 \\ \mathrm{H} & 3.77252900 & -2.15458400 & 0.31072900 \\ \mathrm{H} & -3.99834500 & 1.27278500 & 0.45617800 \\ \mathrm{C} & -2.04526200 & -0.44221100 & -0.46732500 \\ \mathrm{H} & -2.54929600 & -0.47754600 & -1.43758100 \\ \mathrm{~N} & -1.26571900 & 0.82627400 & -0.38964000 \\ \mathrm{H} & -1.70322100 & 1.74187300 & -0.37057500\end{array}$

8-NHCH3-N7H Guanine Radical (3)

\begin{tabular}{lrcr}
\hline SCF Done: & E(UB3LYP) $=$ & -637.876679870 a.u. \\
02 & & & \\
$\mathrm{C}$ & -4.14475500 & 0.20511400 & 0.45774600 \\
$\mathrm{H}$ & -4.82657600 & -0.01351700 & 1.28175400 \\
$\mathrm{H}$ & -4.66310600 & -0.01149900 & -0.48044200 \\
$\mathrm{~N}$ & -2.96310000 & -0.66506300 & 0.57353900 \\
$\mathrm{H}$ & -2.50329700 & -0.51368400 & 1.47110500 \\
$\mathrm{C}$ & 0.07494400 & 0.56814000 & -0.22304600 \\
$\mathrm{C}$ & 0.28073400 & -0.84181000 & -0.23277900 \\
$\mathrm{C}$ & 2.47094500 & -0.52677900 & 0.13693100 \\
$\mathrm{C}$ & 1.20529700 & 1.43960600 & -0.01746300 \\
$\mathrm{H}$ & -1.08748600 & -2.42793700 & -0.41926200 \\
$\mathrm{~N}$ & 2.40710000 & 0.81788600 & 0.15886800
\end{tabular}




$\begin{array}{lrrr}\mathrm{N} & 1.45185900 & -1.42588100 & -0.05802500 \\ \mathrm{O} & 1.08339100 & 2.69975400 & -0.00275500 \\ \mathrm{~N} & -0.91766000 & -1.43082400 & -0.44889400 \\ \mathrm{~N} & 3.68657000 & -1.08167000 & 0.32499500 \\ \mathrm{H} & 4.48662800 & -0.49669500 & 0.51726100 \\ \mathrm{H} & 3.78414300 & -2.08588600 & 0.36263400 \\ \mathrm{H} & -3.90960100 & 1.27802300 & 0.48620600 \\ \mathrm{C} & -2.00926400 & -0.46028300 & -0.48556400 \\ \mathrm{H} & -2.55123200 & -0.50493800 & -1.43489000 \\ \mathrm{~N} & -1.23403000 & 0.80806000 & -0.43642300 \\ \mathrm{H} & -1.67918200 & 1.71661800 & -0.37569200\end{array}$

\begin{tabular}{lrrr}
\hline \multicolumn{4}{c}{ 8-NHCH3-N1H Guanine Radical (4) } \\
\hline SCF Done: & E(UB3LYP) $=$ & -637.876771635 a.u. \\
0 2 & & & \\
$\mathrm{C}$ & -4.24630200 & 0.20938900 & 0.34153500 \\
$\mathrm{H}$ & -4.95699200 & 0.01190500 & 1.14731700 \\
$\mathrm{H}$ & -4.71965300 & -0.07162200 & -0.60395900 \\
$\mathrm{~N}$ & -3.04765500 & -0.62036100 & 0.55192200 \\
$\mathrm{H}$ & -2.64782700 & -0.39482000 & 1.46290900 \\
$\mathrm{C}$ & -0.01071500 & 0.58852500 & -0.20061200 \\
$\mathrm{C}$ & 0.22705900 & -0.82294300 & -0.20389600 \\
$\mathrm{C}$ & 2.44387300 & -0.62000400 & 0.10775900 \\
$\mathrm{C}$ & 1.11420100 & 1.45098000 & -0.03159100 \\
$\mathrm{H}$ & 3.15767100 & 1.31157400 & 0.22974000 \\
$\mathrm{H}$ & -1.13476800 & -2.40713500 & -0.38649300 \\
$\mathrm{~N}$ & 2.32409300 & 0.74296000 & 0.10308800 \\
$\mathrm{~N}$ & 1.40389000 & -1.44482100 & -0.05220000 \\
$\mathrm{O}$ & 1.12770000 & 2.70287200 & 0.00407900 \\
$\mathrm{~N}$ & -0.96582700 & -1.40963800 & -0.37283500 \\
$\mathrm{~N}$ & 3.67550300 & -1.13388700 & 0.26182800 \\
$\mathrm{H}$ & 4.46679200 & -0.54390200 & 0.48195400 \\
$\mathrm{H}$ & 3.77446300 & -2.13520300 & 0.35724300 \\
$\mathrm{H}$ & -4.04479800 & 1.28824000 & 0.31989700 \\
$\mathrm{C}$ & -2.02090900 & -0.38434800 & -0.45236700 \\
$\mathrm{H}$ & -2.51702200 & -0.43615900 & -1.42906800 \\
$\mathrm{~N}$ & -1.31039200 & 0.89782700 & -0.34567900 \\
& & &
\end{tabular}

8- $\mathrm{NH}_{2} \mathrm{CH}_{3}$ oxidized Guanine Cation (5)

SCF Done: $\mathrm{E}(\mathrm{RB} 3 \mathrm{LYP})=-637.719150058$ a.u.

11

C

C

$\mathrm{C}$

$\mathrm{C}$

$\mathrm{H}$

$\mathrm{H}$

$\mathrm{N}$

$\mathrm{N}$

$\mathrm{O}$

$\mathrm{N}$

$\mathrm{N}$

$\begin{array}{lrrr}\mathrm{H} & -4.35690900 & -1.13915200 & -0.28815200 \\ \mathrm{H} & -3.36039400 & -2.57076300 & -0.14428100 \\ \mathrm{~N} & 3.03899200 & 0.28249400 & -0.47626600 \\ \mathrm{H} & 3.42519100 & 1.23056800 & -0.37930700 \\ \mathrm{H} & 2.66455300 & 0.20505700 & -1.43106900 \\ \mathrm{C} & 4.14970300 & -0.71037500 & -0.28485900 \\ \mathrm{H} & 4.52955100 & -0.60660600 & 0.73079900 \\ \mathrm{H} & 3.76318400 & -1.71312900 & -0.45957700 \\ \mathrm{H} & 4.92453100 & -0.47215400 & -1.01193200\end{array}$

\begin{tabular}{lrrc}
\hline \multicolumn{4}{c}{ 8-NHCH3-N7H oxidized Guanine Cation (6) } \\
\hline SCF Done: & E(RB3LYP) & -637.699239779 & a.u. \\
11 & & & \\
$\mathrm{C}$ & 0.18495300 & 0.70126700 & -0.12440300 \\
$\mathrm{C}$ & 0.13560400 & -0.77039100 & -0.16385000 \\
$\mathrm{C}$ & 2.37275400 & -0.79386000 & 0.07769800 \\
$\mathrm{C}$ & 1.50796400 & 1.38259000 & 0.03500600 \\
$\mathrm{C}$ & -2.02265800 & 0.09677300 & -0.41174600 \\
$\mathrm{H}$ & -2.41436100 & 0.16597300 & -1.43213800 \\
$\mathrm{H}$ & -1.51525300 & -2.03624000 & -0.39730300 \\
$\mathrm{~N}$ & 2.55568500 & 0.54902500 & 0.12737300 \\
$\mathrm{~N}$ & 1.19746200 & -1.51675900 & -0.06500100 \\
$\mathrm{O}$ & 1.55464800 & 2.62300000 & 0.06931900 \\
$\mathrm{~N}$ & -1.00233200 & 1.16725800 & -0.24999600 \\
$\mathrm{~N}$ & -1.14868400 & -1.09261800 & -0.31212600 \\
$\mathrm{~N}$ & 3.46284200 & -1.54115600 & 0.17410800 \\
$\mathrm{H}$ & 4.37015600 & -1.10384300 & 0.28066800 \\
$\mathrm{H}$ & 3.39319400 & -2.55106000 & 0.14151300 \\
$\mathrm{~N}$ & -3.08116400 & 0.24208900 & 0.51714000 \\
$\mathrm{H}$ & -2.74497800 & 0.06951100 & 1.46385800 \\
$\mathrm{C}$ & -4.25467100 & -0.60439700 & 0.22605200 \\
$\mathrm{H}$ & -4.64272100 & -0.35556300 & -0.76441600 \\
$\mathrm{H}$ & -4.03368800 & -1.67837300 & 0.26346300 \\
$\mathrm{H}$ & -5.02518800 & -0.38088600 & 0.96519100 \\
$\mathrm{H}$ & -1.25467900 & 2.15971500 & -0.24842000
\end{tabular}

8-NHCH 3 oxidized Guanine neutral (7)

SCF Done: $\mathrm{E}(\mathrm{RB} 3 \mathrm{LYP})=-637.273431877$ a.u.

01

$\begin{array}{lrcc}\mathrm{C} & -4.22987900 & 0.25566800 & 0.16468700 \\ \mathrm{H} & -5.02749800 & 0.04076000 & 0.87896100 \\ \mathrm{H} & -4.58943200 & -0.00257300 & -0.83515100 \\ \mathrm{~N} & -3.06961100 & -0.58588000 & 0.50000000 \\ \mathrm{H} & -2.76334100 & -0.37611100 & 1.44983600 \\ \mathrm{C} & 0.01763000 & 0.56455400 & -0.14015400 \\ \mathrm{C} & 0.26890500 & -0.88845100 & -0.10436400 \\ \mathrm{C} & 2.47046000 & -0.51632300 & 0.07767900 \\ \mathrm{C} & 1.20588500 & 1.45734000 & 0.00142400 \\ \mathrm{H} & -1.11393900 & -2.44901500 & -0.26460300 \\ \mathrm{~N} & 2.40167600 & 0.83456600 & 0.09408900 \\ \mathrm{~N} & 1.45083900 & -1.44403300 & -0.00383300 \\ \mathrm{O} & 1.07883700 & 2.69898400 & 0.02570200 \\ \mathrm{~N} & -0.93162700 & -1.45414300 & -0.19358100\end{array}$




$\begin{array}{lrrr}\mathrm{N} & 3.69353600 & -1.04351100 & 0.15350600 \\ \mathrm{H} & 4.50050000 & -0.43768300 & 0.21901700 \\ \mathrm{H} & 3.81895000 & -2.04682200 & 0.14706800 \\ \mathrm{H} & -4.02080500 & 1.33208500 & 0.19338800 \\ \mathrm{C} & -1.95940500 & -0.42412400 & -0.39651600 \\ \mathrm{H} & -2.33363000 & -0.47985800 & -1.42481600 \\ \mathrm{~N} & -1.22239500 & 0.85519700 & -0.29101800\end{array}$

\begin{tabular}{lrrc}
\hline \multicolumn{4}{c}{ 8-NH $\mathbf{N H}_{\mathbf{2}} \mathbf{C H}_{\mathbf{3}}$ oxidized Guanine neutral (7') } \\
\hline SCF Done: & E(RB3LYP) & -637.241528765 a.u. \\
$0 \mathrm{~N}$ & & & \\
$\mathrm{C}$ & 0.09730700 & 0.53929300 & -0.14382800 \\
$\mathrm{C}$ & 0.36343200 & -0.90585900 & -0.08835800 \\
$\mathrm{C}$ & 2.60537500 & -0.52624300 & 0.10269100 \\
$\mathrm{C}$ & 1.27041300 & 1.44567500 & 0.00310600 \\
$\mathrm{C}$ & -1.83139700 & -0.46158800 & -0.47632200 \\
$\mathrm{H}$ & -2.25709700 & -0.55936000 & -1.47836300 \\
$\mathrm{H}$ & -0.99750800 & -2.44792600 & -0.50234300 \\
$\mathrm{~N}$ & 2.45886500 & 0.85336000 & 0.10564600 \\
$\mathrm{~N}$ & 1.52361300 & -1.45225900 & 0.02605500 \\
$\mathrm{O}$ & 1.09815300 & 2.69898400 & 0.01371800 \\
$\mathrm{~N}$ & -1.14474700 & 0.81785100 & -0.33837400 \\
$\mathrm{~N}$ & -0.86536500 & -1.49468200 & -0.18083100 \\
$\mathrm{~N}$ & 3.80925000 & -1.00594700 & 0.18160600 \\
$\mathrm{H}$ & 3.74816800 & -2.02551200 & 0.16922800 \\
$\mathrm{~N}$ & -2.99538400 & -0.53192100 & 0.48142400 \\
$\mathrm{H}$ & -3.36657200 & -1.48900800 & 0.44154900 \\
$\mathrm{C}$ & -4.11290000 & 0.42499000 & 0.18860200 \\
$\mathrm{H}$ & -4.91543200 & 0.21638900 & 0.89479100 \\
$\mathrm{H}$ & -3.74999900 & 1.44218700 & 0.31536600 \\
$\mathrm{H}$ & -4.45147500 & 0.25518300 & -0.83296500 \\
$\mathrm{H}$ & -2.65231300 & -0.38625400 & 1.43896300
\end{tabular}

8-NHCH $\mathrm{NH}_{3}$ oxidized Guanine neutral (8)

\begin{tabular}{lrrr}
\hline SCF Done: & E(RB3LYP $)=$ & -637.317515832 a.u. \\
01 & & & \\
$\mathrm{C}$ & 4.14533600 & -0.82149600 & 0.28004200 \\
$\mathrm{H}$ & 5.19626700 & -0.55424300 & 0.16618500 \\
$\mathrm{H}$ & 3.96623900 & -1.72101300 & -0.31448100 \\
$\mathrm{~N}$ & 3.33539100 & 0.29543000 & -0.21913100 \\
$\mathrm{H}$ & 3.69387600 & 1.20717300 & 0.04343900 \\
$\mathrm{C}$ & -0.10927900 & 0.76646500 & -0.04750400 \\
$\mathrm{C}$ & -0.06559400 & -0.62528500 & -0.07343300 \\
$\mathrm{C}$ & -2.27814100 & -0.90922900 & 0.00862300 \\
$\mathrm{C}$ & -1.37570700 & 1.39480200 & 0.03517800 \\
$\mathrm{C}$ & 1.96976700 & 0.25201800 & -0.11247500 \\
$\mathrm{H}$ & -3.36109900 & 0.84723200 & 0.10650600 \\
$\mathrm{H}$ & 1.66191600 & -1.87761900 & -0.14063200 \\
$\mathrm{~N}$ & -2.42442300 & 0.45527000 & 0.05272300 \\
$\mathrm{~N}$ & -1.09423500 & -1.50034800 & -0.04756600 \\
$\mathrm{O}$ & -1.64352400 & 2.61855200 & 0.09397100 \\
$\mathrm{~N}$ & 1.17397600 & 1.30735800 & -0.06818700 \\
$\mathrm{~N}$ & 1.27046400 & -0.94361000 & -0.11679400 \\
$\mathrm{~N}$ & -3.41726100 & -1.65363500 & -0.02536100
\end{tabular}

$\begin{array}{lrrr}\mathrm{H} & -4.27734800 & -1.23176400 & 0.30300400 \\ \mathrm{H} & -3.30727600 & -2.63942300 & 0.17551300 \\ \mathrm{H} & 3.94993200 & -1.04566800 & 1.33632000\end{array}$

\begin{tabular}{lrrr}
\hline \multicolumn{4}{c}{ 8-NHCH3 Oxidized Guanine Radical Neutral(9) } \\
\hline SCF Done: & E(UB3LYP) & -636.682639940 a.u. \\
02 & & & \\
$\mathrm{C}$ & -0.00908800 & 0.54542700 & 0.00326600 \\
$\mathrm{C}$ & -0.27906300 & -0.85687300 & 0.00430600 \\
$\mathrm{C}$ & -2.47851800 & -0.49386000 & -0.00142500 \\
$\mathrm{C}$ & -1.15259000 & 1.44420800 & 0.00135100 \\
$\mathrm{C}$ & 1.88917100 & -0.43580800 & 0.00215100 \\
$\mathrm{H}$ & 1.15846200 & -2.44886400 & 0.00310300 \\
$\mathrm{~N}$ & -2.37983600 & 0.84525500 & -0.00151900 \\
$\mathrm{~N}$ & -1.46050000 & -1.42794400 & 0.00258900 \\
$\mathrm{O}$ & -1.02339400 & 2.69776300 & 0.00165000 \\
$\mathrm{~N}$ & 1.31749400 & 0.79396500 & 0.00265900 \\
$\mathrm{~N}$ & 0.95764400 & -1.45435600 & 0.00571800 \\
$\mathrm{~N}$ & -3.71647700 & -1.01960200 & -0.00555100 \\
$\mathrm{H}$ & -4.52243900 & -0.41037100 & -0.01156000 \\
$\mathrm{H}$ & -3.84426200 & -2.02180000 & -0.00825700 \\
$\mathrm{~N}$ & 3.19561900 & -0.66796400 & -0.00571900 \\
$\mathrm{H}$ & 3.50111200 & -1.63471000 & 0.01130700 \\
$\mathrm{C}$ & 4.20930800 & 0.38451600 & -0.00517900 \\
$\mathrm{H}$ & 4.05522600 & 1.06690400 & -0.84369500 \\
$\mathrm{H}$ & 4.18452200 & 0.95080800 & 0.92968600 \\
$\mathrm{H}$ & 5.18159400 & -0.09521100 & -0.10784600
\end{tabular}

8-NCH3 Oxidized Guanine Neutral (10)

SCF Done: $\mathrm{E}(\mathrm{RB} 3 \mathrm{LYP})=-636.054805514$ a.u.

01

$\begin{array}{llll}\text { C } & 0.05562400 & 0.53390600 & -0.00408200\end{array}$

$\begin{array}{llll}\text { C } & -0.23268000 & -0.90946300 & -0.00173800\end{array}$

$\begin{array}{llll}\text { C } & -2.42918200 & -0.47977500 & 0.00121300\end{array}$

$\begin{array}{llll}\text { C } & -1.11120200 & 1.45824100 & -0.00022700\end{array}$

$\begin{array}{llll}\text { C } & 1.96389300 & -0.48555800 & -0.00337100\end{array}$

$\begin{array}{llll}\mathrm{H} & 1.17275300 & -2.49097900 & -0.00046600\end{array}$

$\begin{array}{llll}\mathrm{N} & -2.32727600 & 0.86439000 & 0.00220100\end{array}$

$\mathrm{N} \quad \begin{array}{llll}\mathrm{N} & -1.42114600 & -1.43874700 & -0.00028400\end{array}$

$\begin{array}{llll}\mathrm{O} & -0.94873500 & 2.69370600 & 0.00161900\end{array}$

$\begin{array}{llll}\mathrm{N} & 1.32196300 & 0.78568800 & -0.00799800\end{array}$

$\mathrm{N} \quad \begin{array}{llll}0.97736600 & -1.49538400 & -0.00206100\end{array}$

$\begin{array}{llll}\mathrm{N} & -3.65861600 & -0.98905100 & 0.00236000\end{array}$

$\begin{array}{llll}\mathrm{H} & -4.46046300 & -0.37168100 & 0.00408400\end{array}$

$\mathrm{H} \quad-3.79634300 \quad-1.99152700 \quad 0.00185200$

$\begin{array}{llll}\mathrm{N} & 3.21070800 & -0.74797700 & 0.00405800\end{array}$

$\begin{array}{llll}\mathrm{C} & 4.18013600 & 0.33175700 & 0.00366100\end{array}$

$\begin{array}{llll}\mathrm{H} & 3.74753000 & 1.33166300 & -0.06279000\end{array}$

$\begin{array}{llll}\mathrm{H} & 4.77898600 & 0.25330900 & 0.91690900\end{array}$

$\begin{array}{lllll}\mathrm{H} & 4.86688300 & 0.17247900 & -0.83320200\end{array}$

8- $\mathrm{NCH}_{3}-5-\mathrm{NHCH}_{3}$ Oxidized Guanine Neutral (11)

SCF Done: $\mathrm{E}(\mathrm{RB} 3 \mathrm{LYP})=-731.949850307$ a.u. 
01

$\mathrm{C}$

C

C

C

$\mathrm{H}$

$\mathrm{N}$

$\mathrm{N}$

$\mathrm{O}$

$\mathrm{N}$

$\mathrm{N}$

$\mathrm{N}$

$\mathrm{H}$

$\mathrm{H}$

$\mathrm{N}$

C

$\mathrm{H}$

$\mathrm{H}$

$\mathrm{H}$

$\mathrm{H}$

$\mathrm{N}$

C

$\mathrm{H}$

$\mathrm{H}$

$\mathrm{H}$

$\mathrm{H}$ $\begin{array}{llll}0.13813800 & 0.56959800 & -0.00871200\end{array}$

$\begin{array}{lll}-0.05315800 & -0.91710300 & -0.20525400\end{array}$

$\begin{array}{llll}2.15699700 & -1.24962100 & 0.00527600\end{array}$

$\begin{array}{lll}1.38894000 & 0.76677900 & 0.86153500\end{array}$

$\begin{array}{lll}1.2 .09114500 & -0.01665000 & 0.24487900\end{array}$

$\begin{array}{lll}-1.78770300 & -2.08119200 & -0.27836500\end{array}$

$\begin{array}{lll}2.40947300 & -0.10622500 & 0.65864100\end{array}$

$\begin{array}{llll}0.90654800 & -1.78082600 & -0.30758400\end{array}$

$\begin{array}{llll}1.47789200 & 1.74389800 & 1.62409100\end{array}$

$\begin{array}{lll}-1.12817300 & 0.92643800 & 0.60581300\end{array}$

$\begin{array}{lll}-1.37665100 & -1.15633800 & -0.20275700\end{array}$

$3.18654900-2.03283000 \quad-0.31124300$

$\begin{array}{llll}4.12788800 & -1.75224300 & -0.06950900\end{array}$

$\begin{array}{llll}3.02988500 & -2.93380900 & -0.74255600\end{array}$

$\begin{array}{lll}-3.35238300 & 0.13217800 & 0.36259700\end{array}$

$\begin{array}{lll}-4.21502300 & -0.99006100 & -0.00530500\end{array}$

$\begin{array}{lll}-4.17758900 & -1.78575800 & 0.74912100\end{array}$

$\begin{array}{lll}-5.24686400 & -0.63955000 & -0.06023200\end{array}$

$\begin{array}{llll}-3.95880600 & -1.42784300 & -0.97796800\end{array}$

$\begin{array}{lll}-1.41455800 & 1.89822800 & 0.56189900\end{array}$

$\begin{array}{llll}0.30596000 & 1.19635900 & -1.34366600\end{array}$

$\begin{array}{llll}0.63734600 & 2.63270800 & -1.31913000\end{array}$

$\begin{array}{llll}1.59852800 & 2.86496000 & -0.84352400\end{array}$

$\begin{array}{llll}0.67483900 & 2.98092300 & -2.35398500\end{array}$

$\begin{array}{llll}-0.15085300 & 3.19331400 & -0.81032900\end{array}$

$\begin{array}{llll}1.05027000 & 0.70658800 & -1.83962100\end{array}$

\begin{tabular}{lrrr}
\hline \multicolumn{5}{c}{ 5,8-NCH3 Oxidized Guanine Anion (11AN) } \\
SCF Done: & E(RB3LYP) & -731.452350959 & a.u. \\
$-1 ~ 1$ & & & \\
$\mathrm{C}$ & -0.14802700 & 0.59956800 & 0.06663200 \\
$\mathrm{C}$ & 0.05129900 & -0.88527700 & 0.21770600 \\
$\mathrm{C}$ & -2.15109500 & -1.26902400 & 0.00749100 \\
$\mathrm{C}$ & -1.41010800 & 0.79279100 & -0.76507500 \\
$\mathrm{C}$ & 2.08191000 & -0.00032900 & -0.27475600 \\
$\mathrm{~N}$ & -2.42868200 & -0.10327200 & -0.58988300 \\
$\mathrm{~N}$ & -0.89385600 & -1.77380100 & 0.31257500 \\
$\mathrm{O}$ & -1.57669900 & 1.82236800 & -1.46212800 \\
$\mathrm{~N}$ & 1.37433100 & -1.13062300 & 0.19672400 \\
$\mathrm{~N}$ & -3.16638800 & -2.09516400 & 0.28400000 \\
$\mathrm{H}$ & -4.10990500 & -1.83774200 & 0.02543000 \\
$\mathrm{H}$ & -2.98343000 & -3.02126500 & 0.64772700 \\
$\mathrm{~N}$ & 1.13275300 & 0.94944100 & -0.63599500 \\
$\mathrm{H}$ & 1.78410400 & -2.05831300 & 0.25419600 \\
$\mathrm{C}$ & -0.57992200 & 2.63778200 & 1.28168900 \\
$\mathrm{H}$ & -0.59065700 & 3.03832100 & 2.30221700 \\
$\mathrm{H}$ & -1.54031300 & 2.94354100 & 0.82378800 \\
$\mathrm{~N}$ & -0.35257600 & 1.19550600 & 1.36036900 \\
$\mathrm{C}$ & 4.17001400 & -1.02458300 & 0.01986000 \\
$\mathrm{H}$ & 3.96778800 & -1.91876900 & -0.58398500 \\
$\mathrm{H}$ & 4.01183200 & -1.28339600 & 1.07418500 \\
$\mathrm{~N}$ & 3.35179300 & 0.11803000 & -0.39856000
\end{tabular}

\begin{tabular}{|c|c|c|c|}
\hline $\mathrm{H}$ & 5.22425000 & -0.77235100 & -0.10746200 \\
\hline & 0.20714400 & 3.19474800 & 0.73780700 \\
\hline & 1.43673900 & 1.90989500 & -0.50277300 \\
\hline
\end{tabular}

\section{8-NCH3-5-NHCH3 Oxidized Guanine Anion (11'AN)}

SCF Done: $\mathrm{E}(\mathrm{RB} 3 \mathrm{LYP})=-731.478253296$ a.u.

$-11$

$\begin{array}{llll}\text { C } & -0.09415800 & 0.60542400 & 0.02404000\end{array}$

$\begin{array}{llll}\text { C } & 0.12476600 & -0.87135100 & 0.26551400\end{array}$

$\begin{array}{llll}\text { C } & -2.05261100 & -1.30557200 & -0.02999900\end{array}$

$\begin{array}{llll}\text { C } & -1.30460600 & 0.71214600 & -0.91730600\end{array}$

$\begin{array}{llll}\text { C } & 2.08902100 & 0.14979300 & -0.20061200\end{array}$

$\begin{array}{llll}\mathrm{H} & 1.89106200 & -1.96189200 & 0.36999500\end{array}$

N $\quad-2.29913300 \quad-0.20047800 \quad-0.76327300$

$\begin{array}{llll}\mathrm{N} & -0.81750000 & -1.77295500 & 0.37994800\end{array}$

$\begin{array}{lllll}\mathrm{O} & & -1.41320000 & 1.66049400 & -1.72566500\end{array}$

$\begin{array}{llll}\mathrm{N} & 1.17185700 & 1.10146200 & -0.45668400\end{array}$

$\begin{array}{llll}\mathrm{N} & 1.44091700 & -1.05636000 & 0.28529100\end{array}$

$\begin{array}{llll}\mathrm{N} & -3.08723300 & -2.09753600 & 0.25848300\end{array}$

$\mathrm{H} \quad-4.01497500 \quad-1.85547900 \quad-0.06499400$

$\begin{array}{llll}\mathrm{H} & -2.93941500 & -2.97316800 & 0.74368900\end{array}$

$\begin{array}{llll}\mathrm{N} & 3.38152300 & 0.23527000 & -0.36905000\end{array}$

$\begin{array}{llll}\mathrm{C} & 4.18011700 & -0.94752400 & -0.04735600\end{array}$

$\mathrm{H} \quad 3.95717200 \quad-1.80927500 \quad-0.69473000$

$\mathrm{H} \quad 5.23796300 \quad-0.71154000 \quad-0.18891700$

$\begin{array}{llll}\mathrm{H} & 4.06263600 & -1.28149600 & 0.99414200\end{array}$

$\begin{array}{llll}\mathrm{N} & -0.46738900 & 1.21058000 & 1.35598700\end{array}$

$\begin{array}{llll}\text { C } & -0.91516600 & 2.61425000 & 1.27984200\end{array}$

$\begin{array}{llll}\mathrm{H} & -1.84168000 & 2.75362500 & 0.70743000\end{array}$

$\begin{array}{llll}\mathrm{H} & -1.08795600 & 2.97087500 & 2.29897100\end{array}$

$\begin{array}{llll}\mathrm{H} & -0.13400600 & 3.23410700 & 0.83565600\end{array}$

$\begin{array}{llll}\mathrm{H} & -1.25066900 & 0.66741200 & 1.72442500\end{array}$

\begin{tabular}{lrrr}
\hline \multicolumn{4}{c}{$\mathbf{5 , 8 - d i N C H 3}$ Sp Anion (12AN) } \\
SCF Done: & E(RB3LYP) $=$ & -731.511346788 a.u. \\
-11 & & & \\
$\mathrm{C}$ & 3.47918400 & -2.01350600 & -0.32203300 \\
$\mathrm{H}$ & 4.54401500 & -2.20332700 & -0.17342000 \\
$\mathrm{H}$ & 3.21325800 & -2.37314300 & -1.32515500 \\
$\mathrm{~N}$ & 3.22170100 & -0.58009000 & -0.16536600 \\
$\mathrm{C}$ & 0.19995300 & 1.22582500 & -0.13206800 \\
$\mathrm{C}$ & 1.98763600 & -0.22199600 & -0.23214200 \\
$\mathrm{~N}$ & 0.85149700 & -0.98006200 & -0.45085100 \\
$\mathrm{H}$ & 0.87048600 & -1.93971300 & -0.11888300 \\
$\mathrm{~N}$ & 1.57043700 & 1.10231300 & -0.12163000 \\
$\mathrm{C}$ & -0.36390800 & -0.19994900 & -0.18708700 \\
$\mathrm{~N}$ & -1.42783000 & -0.39877400 & -1.15151500 \\
$\mathrm{C}$ & -1.02315000 & -0.58891700 & 1.17306700 \\
$\mathrm{O}$ & -0.40205900 & -0.59440100 & 2.26630900 \\
$\mathrm{~N}$ & -2.30236900 & -0.90629600 & 0.95940600 \\
$\mathrm{C}$ & -2.46262600 & -0.76347700 & -0.41727200 \\
$\mathrm{~N}$ & -3.69927700 & -0.96990100 & -0.93672300 \\
$\mathrm{H}$ & -4.36165800 & -1.48751200 & -0.37351900 \\
$\mathrm{H}$ & -3.77674300 & -1.07793700 & -1.94027900
\end{tabular}




$\begin{array}{lrrr}\mathrm{C} & 0.23796300 & 3.55517400 & -0.02008900 \\ \mathrm{H} & -0.46297200 & 4.39172800 & -0.02456800 \\ \mathrm{H} & 0.91694900 & 3.67638600 & -0.87470400 \\ \mathrm{~N} & -0.50567900 & 2.29410500 & -0.08717600 \\ \mathrm{H} & 0.83907300 & 3.62210300 & 0.89640400 \\ \mathrm{H} & 2.92664000 & -2.62209800 & 0.40676100 \\ \mathrm{H} & 2.21775800 & 1.88073300 & -0.10037200\end{array}$

\section{5-NCH3-8-NCH3 Oxidized Guanine Anion (12AN')}

SCF Done: $\mathrm{E}(\mathrm{RB} 3 \mathrm{LYP})=-731.519959370$ a.u.

$-11$

$\begin{array}{llll}\text { C } & 3.64891700 & -1.73211000 & -0.31526500\end{array}$

$\mathrm{H} \quad 4.72761100 \quad-1.84650400 \quad-0.18856200$

$\begin{array}{llll}\mathrm{H} & 3.38558900 & -2.13571100 & -1.30277000\end{array}$

$\begin{array}{lllll}\mathrm{N} & 3.29207300 & -0.31970400 & -0.18234700\end{array}$

$\begin{array}{lllll}\text { C } & 0.23031700 & 1.17122700 & -0.13000700\end{array}$

$\begin{array}{llll}\mathrm{C} & 2.03190800 & -0.04547600 & -0.25587700\end{array}$

$\begin{array}{llll}\mathrm{N} & 0.98299000 & -0.94162700 & -0.48002300\end{array}$

$\begin{array}{llll}\mathrm{H} & 1.09486300 & -1.88234400 & -0.11264900\end{array}$

$\begin{array}{lllll}\mathrm{N} & 1.54190500 & 1.26310800 & -0.15394000\end{array}$

$\begin{array}{llll}\mathrm{C} & -0.28025700 & -0.26867800 & -0.19829200\end{array}$

$\begin{array}{llll}\mathrm{N} & -1.34691200 & -0.49589600 & -1.15773500\end{array}$

$\begin{array}{llll}\text { C } & -0.91127800 & -0.70667200 & 1.16154400\end{array}$

$\begin{array}{llll}\mathrm{O} & -0.28029800 & -0.70668400 & 2.24845600\end{array}$

$\begin{array}{llll}\mathrm{N} & -2.18073300 & -1.06553200 & 0.95351800\end{array}$

$\begin{array}{llll}\mathrm{C} & -2.36058400 & -0.90754800 & -0.41807200\end{array}$

$\begin{array}{llll}\mathrm{N} & -3.59530000 & -1.15107800 & -0.92841200\end{array}$

$\mathrm{H} \quad-4.22462200-1.71049800 \quad-0.36682200$

$\begin{array}{llll}\mathrm{H} & -3.67361500 & -1.25929300 & -1.93210400\end{array}$

$\begin{array}{llll}\text { C } & -0.18496900 & 3.59022500 & 0.03658400\end{array}$

$\begin{array}{llll}\mathrm{H} & -1.07397400 & 4.20907700 & 0.15844200\end{array}$

$\begin{array}{llll}\mathrm{H} & 0.33809800 & 3.89365300 & -0.87619700\end{array}$

$\begin{array}{llll}\mathrm{N} & -0.61031500 & 2.19775100 & -0.04220000\end{array}$

$\begin{array}{llll}\mathrm{H} & 0.48083300 & 3.74564100 & 0.89067300\end{array}$

$\begin{array}{llll}\mathrm{H} & 3.15849600 & -2.36340500 & 0.43954800\end{array}$

$\begin{array}{llll}\mathrm{H} & -1.60118000 & 1.98790600 & -0.04292600\end{array}$ $\begin{array}{lll}2.17694400 & 1.90799100 & -0.26075200\end{array}$

$\begin{array}{lll}-0.50786800 & 2.21591600 & -0.14895900\end{array}$

$\begin{array}{lll}-1.51445500 & 2.08036400 & -0.11700200\end{array}$

$\begin{array}{llll}0.01156700 & 3.58024500 & -0.28073900\end{array}$

$\begin{array}{llll}0.53171700 & 3.69682800 & -1.23530500\end{array}$

$\begin{array}{lll}-0.83307900 & 4.26539000 & -0.24319200\end{array}$

$\begin{array}{lll}0.69586200 & 3.80470500 & 0.54122800\end{array}$

\section{5,8-diNCH 3 -Sp Tautomer (13)}

SCF Done: $\mathrm{E}(\mathrm{RB} 3 \mathrm{LYP})=-731.969319702$ a.u.

01

$\begin{array}{llll}\text { C } & 3.66622600 & -1.78333800 & -0.36428900\end{array}$

$\mathrm{H} \quad 4.75089100 \quad-1.88167000 \quad-0.29539100$

$\mathrm{H} \quad 3.36056300 \quad-2.17148700 \quad-1.34472600$

N $\quad 3.29964300 \quad-0.37906300 \quad-0.18392100$

$\begin{array}{llll}\mathrm{C} & 0.16277400 & 1.21093900 & -0.08345100\end{array}$

$\begin{array}{llll}\mathrm{C} & 2.04953800 & -0.10754100 & -0.23163200\end{array}$

$\begin{array}{llll}\mathrm{N} & 0.95952400 & -0.94383400 & -0.45102600\end{array}$

$\begin{array}{llll}\mathrm{H} & 1.04489800 & -1.90833000 & -0.14798000\end{array}$

$\begin{array}{llll}\mathrm{N} & 1.53854500 & 1.18231000 & -0.09123000\end{array}$

$\begin{array}{llll}\text { C } & -0.28472400 & -0.25514200 & -0.16055600\end{array}$

$\mathrm{N} \quad \begin{array}{llll}\mathrm{N} & -1.36161200 & -0.51439600 & -1.09363600\end{array}$

$\begin{array}{lllll}\mathrm{H} & & -1.43163100 & -0.00916400 & -1.97141800\end{array}$

$\begin{array}{llll}\text { C } & -0.96684100 & -0.72326600 & 1.16857300\end{array}$

$\begin{array}{llll}\mathrm{O} & -0.35245200 & -0.79168200 & 2.23980400\end{array}$

$\begin{array}{llll}\mathrm{N} & -2.26730700 & -1.02944100 & 0.94475500\end{array}$

$\begin{array}{llll}\text { C } & -2.47403900 & -0.83148200 & -0.37286200\end{array}$

$\mathrm{N} \quad-3.66423400 \quad-0.96689300 \quad-0.94006000$

$\mathrm{H} \quad-4.46340900 \quad-1.20587900 \quad-0.36794900$

$\mathrm{H} \quad \begin{array}{llll}-3.79185100 & -0.81868300 & -1.93302000\end{array}$

$\begin{array}{llll}\text { C } & -0.04668300 & 3.53168800 & 0.08299000\end{array}$

$\begin{array}{llll}\mathrm{H} & -0.83917500 & 4.28088400 & 0.11264500\end{array}$

$\begin{array}{llll}\mathrm{H} & 0.59871300 & 3.75516900 & -0.77665200\end{array}$

$\begin{array}{llll}\mathrm{N} & -0.64286200 & 2.19937600 & -0.01058600\end{array}$

$\begin{array}{llll}\mathrm{H} & 0.55723600 & 3.64064400 & 0.99329000\end{array}$

$\begin{array}{llll}\mathrm{H} & 3.22748500 & -2.43298400 & 0.40511500\end{array}$

$\begin{array}{llll}\mathrm{H} & 2.13649900 & 1.99740300 & -0.04506300\end{array}$

\footnotetext{
5-NCH3, $8-\mathrm{NHCH}_{3}$ Sp Neutral (12)

\begin{tabular}{lrrr}
\hline SCF Done: & $E(R B 3 L Y P)=$ & -731.960852955 \\
$0 ~ 1$ & & & \\
$\mathrm{C}$ & 3.65538200 & -1.90495000 & -0.22014700 \\
$\mathrm{H}$ & 4.74061200 & -2.00070500 & -0.27419500 \\
$\mathrm{H}$ & 3.22836300 & -2.46496900 & -1.06234300 \\
$\mathrm{~N}$ & 3.29334400 & -0.48858300 & -0.26863600 \\
$\mathrm{C}$ & 0.23272300 & 1.14060400 & -0.11360100 \\
$\mathrm{C}$ & 2.04954900 & -0.22706200 & -0.14368700 \\
$\mathrm{~N}$ & 0.95003300 & -1.02491900 & 0.06505200 \\
$\mathrm{H}$ & 0.96061400 & -1.98776100 & -0.24961300 \\
$\mathrm{~N}$ & 1.56148200 & 1.10517200 & -0.17024600 \\
$\mathrm{C}$ & -0.30783800 & -0.28000400 & -0.01633900 \\
$\mathrm{~N}$ & -1.17235100 & -0.63732000 & -1.11593800 \\
$\mathrm{C}$ & -1.21051600 & -0.47333500 & 1.26144600 \\
$\mathrm{O}$ & -0.80442700 & -0.26593600 & 2.42501400 \\
$\mathrm{~N}$ & -2.41801800 & -0.87554200 & 0.85775800 \\
$\mathrm{C}$ & -2.32058400 & -0.94032800 & -0.52494500 \\
$\mathrm{~N}$ & -3.42258700 & -1.28693800 & -1.22555400 \\
$\mathrm{H}$ & -4.19620700 & -1.70328200 & -0.72673700 \\
$\mathrm{H}$ & -3.32341800 & -1.51153800 & -2.20611300 \\
$\mathrm{H}$ & 3.31852300 & -2.38504800 & 0.70765500
\end{tabular}
}

\section{5,8-diNHCH3-Sp (14)}

SCF Done: $\mathrm{E}(\mathrm{RB} 3 \mathrm{LYP})=-731.977115787$ a.u.

01

$\mathrm{C}$

$\mathrm{H}$

$\mathrm{H}$

$\mathrm{N}$

$\mathrm{H}$

C

C

$$
\mathrm{N}
$$

$\mathrm{N}$

$\mathrm{H}$

C

$\mathrm{N}$

$\mathrm{H}$

C

$\mathrm{O}$

$\mathrm{N}$

C 


$\begin{array}{cccc}\mathrm{H} & -4.72188500 & -0.27275400 & -0.37922100 \\ \mathrm{H} & -3.98307300 & 0.05410200 & -1.92599000 \\ \mathrm{C} & 0.54963900 & 3.27982700 & 0.32024400 \\ \mathrm{H} & -0.09419900 & 4.14047800 & 0.51393700 \\ \mathrm{H} & 1.13901600 & 3.49447600 & -0.58008900 \\ \mathrm{~N} & -0.27800600 & 2.08604000 & 0.16053400 \\ \mathrm{H} & 1.25235400 & 3.18398500 & 1.15725800 \\ \mathrm{H} & 4.47545900 & 0.13521800 & -1.03948200\end{array}$

\begin{tabular}{|c|c|c|c|}
\hline \multicolumn{4}{|c|}{ 5-NHCH $3-8-\mathrm{NCH}_{3} \mathrm{Sp}$ (A14') } \\
\hline \multicolumn{4}{|c|}{$\begin{array}{l}\text { SCF Done: } E(R B 3 L Y P)=-731.974058335 \text { a.u. } \\
01\end{array}$} \\
\hline $\mathrm{C}$ & 4.23293500 & -0.64763500 & -0.20774900 \\
\hline $\mathrm{H}$ & 5.06433600 & -1.35585100 & -0.18515000 \\
\hline $\mathrm{H}$ & 4.25786400 & -0.07564000 & 0.72738400 \\
\hline $\mathrm{N}$ & 2.98491000 & -1.38784400 & -0.38991900 \\
\hline $\mathrm{C}$ & 0.44149000 & 0.88231800 & -0.06004800 \\
\hline $\mathrm{C}$ & 1.89300600 & -0.71596600 & -0.34973700 \\
\hline $\mathrm{N}$ & 0.63818800 & -1.33005700 & -0.55122200 \\
\hline $\mathrm{H}$ & 0.54397500 & -2.27802200 & -0.20204300 \\
\hline $\mathrm{N}$ & 1.73043900 & 0.65978000 & -0.14746600 \\
\hline $\mathrm{C}$ & -0.39768900 & -0.39599600 & -0.19217900 \\
\hline $\mathrm{N}$ & -1.51656400 & -0.30963100 & -1.11503100 \\
\hline $\mathrm{H}$ & -1.46317000 & 0.20097200 & -1.99060700 \\
\hline $\mathrm{C}$ & -1.17447100 & -0.75152200 & 1.12177200 \\
\hline $\mathrm{O}$ & -0.59251600 & -1.02192000 & 2.17910800 \\
\hline $\mathrm{N}$ & -2.51106500 & -0.72628200 & 0.90101900 \\
\hline $\mathrm{C}$ & -2.66971000 & -0.40360800 & -0.39982300 \\
\hline $\mathrm{N}$ & -3.85956400 & -0.21485200 & -0.95363200 \\
\hline $\mathrm{H}$ & -4.69358900 & -0.30911600 & -0.38947200 \\
\hline $\mathrm{H}$ & -3.95063800 & 0.00947700 & -1.93622300 \\
\hline $\mathrm{C}$ & 0.64213800 & 3.30235600 & 0.29321800 \\
\hline $\mathrm{H}$ & -0.05833200 & 4.11326500 & 0.49035200 \\
\hline $\mathrm{H}$ & 1.20883100 & 3.53100600 & -0.61400000 \\
\hline $\mathrm{N}$ & -0.12403500 & 2.07010400 & 0.13203500 \\
\hline $\mathrm{H}$ & 1.33884400 & 3.21717300 & 1.13184900 \\
\hline $\mathrm{H}$ & 4.42231300 & 0.05260500 & -1.03081200 \\
\hline $\mathrm{H}$ & -1.13266200 & 2.12128100 & 0.20264900 \\
\hline
\end{tabular}

$\begin{array}{lrrr}\mathrm{H} & 3.97190200 & -1.55648800 & -1.06385100 \\ \mathrm{H} & 5.17992900 & -0.60925900 & -0.19120000 \\ \mathrm{H} & 1.41127300 & 2.11198300 & 0.05987900 \\ \mathrm{H} & -1.04649900 & 0.77780900 & 2.09345300 \\ \mathrm{O} & -0.18584500 & 1.02184400 & 1.71198200\end{array}$

\section{8-NCH3-5-OH Oxidized Guanine Neutral (15)} SCF Done: $\mathrm{E}(\mathrm{R} 3 \mathrm{LYP})=-712.508992331$ a.u.

01

$\begin{array}{llll}\text { C } & -0.14999000 & 0.73010800 & 0.30475400\end{array}$

$\begin{array}{lllll}\text { C } & -0.02361200 & -0.76678400 & 0.08591700\end{array}$

$\begin{array}{lllll}\mathrm{C} & -2.25095700 & -0.93863100 & -0.13194900\end{array}$

$\begin{array}{lllll}\text { C } & -1.44399400 & 1.23326500 & -0.34989700\end{array}$

$\begin{array}{llll}\mathrm{C} & 2.03619300 & 0.15427600 & -0.15349100\end{array}$

$\mathrm{H} \quad 1.67828800 \quad-1.96644600 \quad-0.14907900$

$\begin{array}{llll}\mathrm{N} & -2.47543200 & 0.35909900 & -0.40170800\end{array}$

$\mathrm{N} \quad \quad-1.02113200 \quad-1.58439500 \quad-0.01858800$

$\begin{array}{lllll}\mathrm{O} & -1.52878900 & 2.41514800 & -0.72279600\end{array}$

$\begin{array}{lllll}\mathrm{N} & 1.09758500 & 1.19081800 & -0.22956500\end{array}$

$\mathrm{N} \quad \begin{array}{llll}\mathrm{N} & 1.29026900 & -1.04055800 & -0.00128700\end{array}$

$\mathrm{N} \quad-3.30348800 \quad-1.74653300 \quad-0.04626400$

$\mathrm{H} \quad-4.23813000 \quad-1.37353900 \quad-0.15065700$

$\mathrm{H} \quad-3.17363200 \quad-2.73646500 \quad 0.11729700$

$\begin{array}{lllll}\mathrm{N} & 3.29775000 & 0.29570400 & -0.26050900\end{array}$

$\begin{array}{llll}\mathrm{C} & 4.13065400 & -0.90659500 & -0.19454300\end{array}$

$\mathrm{H} \quad 3.94531000 \quad-1.49629800 \quad 0.71138300$
}

\begin{tabular}{lrrr}
\hline \multicolumn{4}{c}{ 8-NCH3-5-O Oxidized Guanine Anion (15AN) } \\
\cline { 2 - 2 } SCF Done: & E(R3LYP) $=$ & -712.054851148 & a.u. \\
-11 & & & \\
$\mathrm{C}$ & 0.16028100 & 0.74706900 & 0.37153100 \\
$\mathrm{C}$ & 0.02128200 & -0.73813800 & 0.09102300 \\
$\mathrm{C}$ & 2.24504100 & -0.93694800 & -0.11691800 \\
$\mathrm{C}$ & 1.44201000 & 1.23773600 & -0.30023200 \\
$\mathrm{C}$ & -2.03242000 & 0.18467600 & -0.15243600 \\
$\mathrm{H}$ & -1.68430000 & -1.93623600 & -0.16411900 \\
$\mathrm{~N}$ & 2.48058800 & 0.36522500 & -0.36405200 \\
$\mathrm{~N}$ & 1.01287300 & -1.57299500 & -0.01745800 \\
$\mathrm{O}$ & 1.56195000 & 2.43128200 & -0.65881800 \\
$\mathrm{~N}$ & -1.10855600 & 1.22727600 & -0.22000800 \\
$\mathrm{~N}$ & -1.28979700 & -1.01157600 & -0.02039400 \\
$\mathrm{~N}$ & 3.29651100 & -1.75229300 & -0.04009300 \\
$\mathrm{H}$ & 4.23332000 & -1.38380400 & -0.14715100 \\
$\mathrm{H}$ & 3.16152200 & -2.74669300 & 0.09081500 \\
$\mathrm{~N}$ & -3.30271400 & 0.30590800 & -0.24477000 \\
$\mathrm{C}$ & -4.10571900 & -0.91867800 & -0.17542100 \\
$\mathrm{H}$ & -5.16266900 & -0.64719700 & -0.16830500 \\
$\mathrm{H}$ & -3.93384300 & -1.56823000 & -1.04294200 \\
$\mathrm{H}$ & -3.90099700 & -1.50220800 & 0.73067700 \\
$\mathrm{O}$ & 0.27829900 & 0.98021000 & 1.73091000 \\
$\mathrm{H}$ & -1.44020200 & 2.10733300 & 0.16643900 \\
& & &
\end{tabular}

8-NCH3 Sp Anion (16AN) 
SCF Done: $\mathrm{E}(\mathrm{R} 3 \mathrm{LYP})=-712.087371678$ a.u.

$-11$

$\begin{array}{lrrr}\mathrm{C} & -0.16370400 & 1.50907100 & -0.09374800 \\ \mathrm{C} & 0.32947000 & 0.05428300 & -0.18716500 \\ \mathrm{C} & 2.42189900 & -0.51324600 & -0.43077900 \\ \mathrm{C} & 0.98465900 & -0.37075800 & 1.16738100 \\ \mathrm{C} & -2.01205700 & 0.16358100 & -0.24011700 \\ \mathrm{~N} & 2.26139400 & -0.68668400 & 0.94133700 \\ \mathrm{~N} & 1.38460000 & -0.13433700 & -1.15815700 \\ \mathrm{O} & 0.36212500 & -0.39584500 & 2.25734300 \\ \mathrm{~N} & -0.92051900 & -0.65325100 & -0.46256500 \\ \mathrm{~N} & 3.65403700 & -0.71178400 & -0.95729400 \\ \mathrm{H} & 4.32662200 & -1.22745700 & -0.40481900 \\ \mathrm{H} & 3.73465700 & -0.78572500 & -1.96355000 \\ \mathrm{~N} & -1.51641900 & 1.47089800 & -0.11347600 \\ \mathrm{H} & -0.99859200 & -1.61884800 & -0.15698900 \\ \mathrm{~N} & -3.26197600 & -0.11337900 & -0.17517400 \\ \mathrm{O} & 0.54432700 & 2.51640700 & -0.00924600 \\ \mathrm{C} & -3.60661200 & -1.52728100 & -0.34210300 \\ \mathrm{H} & -3.08382500 & -2.17179400 & 0.37719500 \\ \mathrm{H} & -4.67940000 & -1.65560300 & -0.18817800 \\ \mathrm{H} & -3.36580500 & -1.88876000 & -1.35033900 \\ \mathrm{H} & -2.11502600 & 2.28955100 & -0.06160000\end{array}$

\begin{tabular}{|c|c|c|c|}
\hline \multicolumn{4}{|c|}{ 8- $\mathrm{NCH}_{3}, 5-\mathrm{OH}$ Sp Anion (16AN') } \\
\hline \multicolumn{4}{|c|}{$\begin{array}{l}\text { SCF Done: } E(R 3 L Y P)=-712.067594694 \text { a.u. } \\
-11\end{array}$} \\
\hline $\mathrm{C}$ & -0.24685400 & 1.43808900 & -0.08267500 \\
\hline $\mathrm{C}$ & 0.29623800 & 0.02574600 & -0.18598400 \\
\hline $\mathrm{C}$ & 2.39119800 & -0.53788600 & -0.43970900 \\
\hline $\mathrm{C}$ & 0.95531900 & -0.41905300 & 1.16259400 \\
\hline $\mathrm{C}$ & -2.01940900 & 0.21263400 & -0.24804200 \\
\hline $\mathrm{N}$ & 2.23077900 & -0.73493400 & 0.92926400 \\
\hline $\mathrm{N}$ & 1.35531400 & -0.14673900 & -1.16103900 \\
\hline $\mathrm{O}$ & 0.33563000 & -0.45564000 & 2.25351200 \\
\hline $\mathrm{N}$ & -0.95880000 & -0.66025200 & -0.47103700 \\
\hline $\mathrm{N}$ & 3.62534400 & -0.72472800 & -0.96885000 \\
\hline $\mathrm{H}$ & 4.29318700 & -1.25896800 & -0.42810300 \\
\hline $\mathrm{H}$ & 3.70265700 & -0.78601800 & -1.97635300 \\
\hline $\mathrm{N}$ & -1.53255900 & 1.53658500 & -0.11484700 \\
\hline $\mathrm{H}$ & -1.06036600 & -1.61903100 & -0.15127600 \\
\hline $\mathrm{N}$ & -3.27570100 & -0.05566400 & -0.19004600 \\
\hline $\mathrm{O}$ & 0.61114200 & 2.44128900 & 0.03746100 \\
\hline $\mathrm{C}$ & -3.63336700 & -1.46588900 & -0.34826500 \\
\hline $\mathrm{H}$ & -3.14208200 & -2.10720500 & 0.39648300 \\
\hline $\mathrm{H}$ & -4.71211600 & -1.58172100 & -0.22811500 \\
\hline $\mathrm{H}$ & -3.36366100 & -1.84757300 & -1.34219800 \\
\hline $\mathrm{H}$ & 0.13881300 & 3.29360100 & 0.09014800 \\
\hline
\end{tabular}

\section{8-NCH 3 Sp Tautomer (16)}

$\begin{array}{lrrr}\text { SCF Done: } & \mathrm{E}(\mathrm{R} 3 \mathrm{LYP})= & -712.495731564 \text { a.u. } \\ 0 \mathrm{1} & & & \\ \mathrm{C} & 3.67782200 & -1.49338600 & -0.36265800 \\ \mathrm{H} & 4.76454500 & -1.55928100 & -0.30264400 \\ \mathrm{H} & 3.36855800 & -1.87264300 & -1.34474200 \\ \mathrm{~N} & 3.27006300 & -0.10258400 & -0.16451300 \\ \mathrm{C} & 0.19201500 & 1.44210000 & -0.08662400 \\ \mathrm{O} & -0.50613000 & 2.52684700 & 0.01265300 \\ \mathrm{C} & 2.01879900 & 0.12851200 & -0.23499500 \\ \mathrm{~N} & 0.92436900 & -0.66804400 & -0.48828000\end{array}$

$\begin{array}{rrc}0.98475300 & -1.65451200 & -0.25855100 \\ 1.49939000 & 1.45022100 & -0.07598800 \\ -0.33119900 & 0.03257600 & -0.20486000 \\ -1.39391800 & -0.11586400 & -1.15725500 \\ -0.98209900 & -0.42456600 & 1.17918300 \\ -0.34351200 & -0.44945900 & 2.24844600 \\ -2.24542000 & -0.76642200 & 0.93222900 \\ -2.41724500 & -0.55038500 & -0.42711100 \\ -3.61785200 & -0.82604900 & -0.96755400 \\ -4.39876200 & -1.03442900 & -0.36260700 \\ -3.80392400 & -0.57784500 & -1.92890100 \\ 3.25601800 & -2.15575200 & 0.40378900 \\ 2.08887800 & 2.27624400 & 0.01448600 \\ -1.46792200 & 2.35119300 & -0.03768900\end{array}$

\section{8-NCH3 Sp (17)}

SCF Done: E(R3LYP) $=-712.543583366$ a.u.

01

C $\quad 3.73284400 \quad-1.35337900 \quad-0.52248800$

$\mathrm{H} \quad 4.82197800 \quad-1.40625600 \quad-0.49194100$

$\mathrm{H} \quad 3.41176000 \quad-1.64167800 \quad-1.53148400$

$\begin{array}{llll}\mathrm{N} & 3.30965000 & 0.00392600 & -0.17640800\end{array}$

$\begin{array}{lllll}\text { C } & 0.14558400 & 1.47709600 & 0.10293500\end{array}$

$\begin{array}{llll}\mathrm{O} & -0.61855100 & 2.42194500 & 0.27457400\end{array}$

$\begin{array}{llll}\mathrm{C} & 2.05241900 & 0.22708900 & -0.19020800\end{array}$

$\mathrm{N} \quad 0.98581300 \quad-0.61033400 \quad-0.49796800$

$\mathrm{H} \quad \begin{array}{llll}1.09755000 & -1.60034100 & -0.30260800\end{array}$

$\begin{array}{llll}\mathrm{N} & 1.49840100 & 1.48693500 & 0.09747500\end{array}$

$\begin{array}{lllll}\mathrm{C} & -0.27285300 & 0.01113000 & -0.14784700\end{array}$

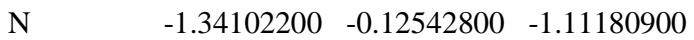

$\mathrm{H} \quad-1.38972500 \quad 0.47155200 \quad-1.93224700$

$\begin{array}{llll}\mathrm{C} & -0.97594000 & -0.58757700 & 1.12164500\end{array}$

O $\quad-0.36815100-0.80067400 \quad 2.17521800$

$\begin{array}{lllll}\mathrm{N} & -2.28369800 & -0.81540700 & 0.85774700\end{array}$

C $\quad-2.47340200 \quad-0.47008700 \quad-0.43077900$

$\mathrm{N} \quad \quad-3.66215900 \quad-0.49134100 \quad-1.01312000$

$\mathrm{H} \quad-4.47662000 \quad-0.74546200 \quad-0.46958500$

$\mathrm{H} \quad \begin{array}{llll}\mathrm{H} & -3.77683300 & -0.23048700 & -1.98434700\end{array}$

$\mathrm{H} \quad 3.34374700 \quad-2.10050900 \quad 0.18165500$

$\begin{array}{llll}\mathrm{H} & 2.06094200 & 2.31890900 & 0.24125700\end{array}$

\begin{tabular}{lrrr}
\hline \multicolumn{4}{c}{ 8-NHCH3 Sp (18) } \\
\hline SCF Done: & E(R3LYP) $=$ & -712.555158014 a.u. \\
01 & & & \\
C & -4.32700500 & 0.00564100 & -0.11406300 \\
$\mathrm{H}$ & -5.13675900 & 0.58271700 & -0.55898700 \\
$\mathrm{H}$ & -4.31663100 & 0.17990900 & 0.96427200 \\
$\mathrm{~N}$ & -3.07699000 & 0.45457700 & -0.72660100 \\
$\mathrm{H}$ & -3.12024500 & 1.17959000 & -1.43316300 \\
$\mathrm{C}$ & -0.32616200 & -1.07546800 & 0.65576400 \\
$\mathrm{O}$ & 0.30021100 & -1.88073600 & 1.35422500 \\
$\mathrm{C}$ & -1.87933300 & 0.00673600 & -0.38447100 \\
$\mathrm{~N}$ & -0.74124500 & 0.49951300 & -0.98211100 \\
$\mathrm{H}$ & -0.73808600 & 1.46537000 & -1.29888600 \\
$\mathrm{~N}$ & -1.67089300 & -0.96628000 & 0.52299500 \\
$\mathrm{C}$ & 0.38103900 & -0.00209300 & -0.22513600 \\
$\mathrm{~N}$ & 1.50423500 & -0.50198300 & -0.98291500 \\
$\mathrm{H}$ & 1.50238300 & -1.45065100 & -1.34676700 \\
$\mathrm{C}$ & 1.09262700 & 1.07047600 & 0.65458600
\end{tabular}




$\begin{array}{llll}\mathrm{O} & 0.46947500 & 1.86475600 & 1.36827500 \\ \mathrm{~N} & 2.43594700 & 0.97519900 & 0.49973800 \\ \mathrm{C} & 2.63766700 & 0.00529400 & -0.41240400 \\ \mathrm{~N} & 3.84297100 & -0.41586000 & -0.76678900 \\ \mathrm{H} & 4.66117800 & -0.01815600 & -0.32476300 \\ \mathrm{H} & 3.95890200 & -1.13510500 & -1.46936700 \\ \mathrm{H} & -4.49940700 & -1.05550700 & -0.30821200\end{array}$

\begin{tabular}{lrrr}
\hline \multicolumn{4}{c}{ 8-OH Guanine Radical Cation (19) } \\
\hline SCF Done: & E(UB3LYP) $=$ & -618.892521252 a.u. \\
12 & & & \\
$\mathrm{C}$ & 0.34760600 & 0.62362900 & -0.13487900 \\
$\mathrm{C}$ & 0.21313900 & -0.80151800 & -0.16249900 \\
$\mathrm{C}$ & -2.02319000 & -0.69467500 & 0.05473500 \\
$\mathrm{C}$ & -0.81687300 & 1.44761800 & 0.00113600 \\
$\mathrm{C}$ & 2.47380800 & -0.28027000 & -0.33206500 \\
$\mathrm{H}$ & -2.85488600 & 1.19401200 & 0.17840500 \\
$\mathrm{~N}$ & -1.98298400 & 0.67659300 & 0.08399000 \\
$\mathrm{~N}$ & -0.92846300 & -1.46771300 & -0.06945200 \\
$\mathrm{O}$ & -0.85017900 & 2.68398200 & 0.04335200 \\
$\mathrm{~N}$ & 1.44457700 & -1.31647000 & -0.30388600 \\
$\mathrm{~N}$ & -3.21165100 & -1.27979400 & 0.15610100 \\
$\mathrm{H}$ & -4.06474500 & -0.74316400 & 0.25905600 \\
$\mathrm{H}$ & -3.26363100 & -2.29046100 & 0.14246500 \\
$\mathrm{~N}$ & 1.64728700 & 0.93238300 & -0.25567000 \\
$\mathrm{H}$ & 1.66889300 & -2.30384600 & -0.35967300 \\
$\mathrm{O}$ & 3.41498400 & -0.38419000 & 0.69755700 \\
$\mathrm{H}$ & 2.96021400 & -0.40364000 & 1.55829500 \\
$\mathrm{H}$ & 3.04567500 & -0.30227200 & -1.26157200 \\
$\mathrm{H}$ & 2.04174100 & 1.86732700 & -0.28040500
\end{tabular}

8-OH Guanine Radical (21)

SCF Done: E(UB3LYP $)=-618.435447844$ a.u.

02

$\begin{array}{lllll}\text { C } & 0.30690600 & 0.61323900 & -0.14253100\end{array}$

$\begin{array}{llll}\text { C } & 0.17604500 & -0.80661100 & -0.17184900\end{array}$

$\begin{array}{llll}\text { C } & -2.04564500 & -0.60396500 & 0.06227300\end{array}$

$\begin{array}{llll}\text { C } & -0.87685000 & 1.42578500 & 0.00491800\end{array}$

$\begin{array}{llll}\mathrm{C} & 2.43971900 & -0.28834800 & -0.33135400\end{array}$

$\begin{array}{lllll}\mathrm{N} & -2.05224500 & 0.74069400 & 0.10134100\end{array}$

$\begin{array}{lllll}\mathrm{N} & & -0.96910000 & -1.44992900 & -0.07379600\end{array}$

$\begin{array}{llll}\mathrm{O} & -0.81851300 & 2.68770100 & 0.04273100\end{array}$

$\mathrm{N} \quad \begin{array}{llll}\mathrm{N} & 1.41873700 & -1.32557700 & -0.32350500\end{array}$

$\mathrm{N} \quad \quad-3.23800900 \quad-1.22283600 \quad 0.16243000$

$\begin{array}{llll}\mathrm{H} & -4.07796600 & -0.68120100 & 0.30695900\end{array}$

$\mathrm{H} \quad \begin{array}{llll}-3.28526100 & -2.23142400 & 0.17357400\end{array}$

$\begin{array}{llll}\mathrm{N} & 1.61303600 & 0.92077300 & -0.27350600\end{array}$

$\mathrm{H} \quad 1.64950600 \quad-2.31042200 \quad-0.36430000$

$\begin{array}{llll}\mathrm{O} & 3.36430800 & -0.38687400 & 0.72441600\end{array}$

$\begin{array}{llll}\mathrm{H} & 2.88534500 & -0.40739700 & 1.57144300\end{array}$

$\mathrm{H} \quad 3.04343100 \quad-0.31155700 \quad-1.24079700$

$\begin{array}{llll}\mathrm{H} & 2.01060300 & 1.85290600 & -0.26354200\end{array}$

\begin{tabular}{lrrr}
\hline \multicolumn{4}{c}{ 8-OH Guanine Radical (22) } \\
\hline SCF Done: & E(UB3LYP) & -618.438556671 a.u. \\
0 2 & & & \\
C & 0.39837500 & 0.63221200 & -0.12428500 \\
$\mathrm{C}$ & 0.23080200 & -0.78941200 & -0.12344100 \\
$\mathrm{C}$ & -2.00673700 & -0.69014600 & 0.05304600 \\
$\mathrm{C}$ & -0.77392800 & 1.44274500 & -0.00437800 \\
$\mathrm{C}$ & 2.44596700 & -0.23039600 & -0.34695000 \\
$\mathrm{~N}$ & -1.95181400 & 0.67732800 & 0.07643000 \\
$\mathrm{~N}$ & -0.91886100 & -1.46481700 & -0.03970600 \\
$\mathrm{O}$ & -0.84491600 & 2.68979500 & 0.02584300 \\
$\mathrm{~N}$ & 1.46393900 & -1.31919300 & -0.20800200 \\
$\mathrm{~N}$ & -3.21651000 & -1.26355800 & 0.11685700 \\
$\mathrm{H}$ & -4.05449600 & -0.71517500 & 0.25971700 \\
$\mathrm{H}$ & -3.27592800 & -2.27109100 & 0.16985000 \\
$\mathrm{~N}$ & 1.68349000 & 1.00512600 & -0.23448500 \\
$\mathrm{H}$ & 1.66799800 & -2.29230100 & -0.40092400 \\
$\mathrm{O}$ & 3.49679300 & -0.32504900 & 0.59354600 \\
$\mathrm{H}$ & 3.13286900 & -0.18268100 & 1.48384900 \\
$\mathrm{H}$ & 2.94508200 & -0.29445000 & -1.31903100 \\
$\mathrm{H}$ & -2.81912500 & 1.20350300 & 0.14982200
\end{tabular}




$\begin{array}{lrrr}\mathrm{N} & -1.92676300 & 0.67091900 & 0.00050700 \\ \mathrm{~N} & -0.90224700 & -1.46695700 & 0.00090100 \\ \mathrm{O} & -0.80393200 & 2.68721900 & 0.00360600 \\ \mathrm{~N} & 1.51619900 & -1.30620800 & 0.00166500 \\ \mathrm{~N} & -3.21485700 & -1.26550600 & -0.05083800 \\ \mathrm{H} & -4.02754900 & -0.70774600 & 0.18032000 \\ \mathrm{H} & -3.26956800 & -2.25519000 & 0.15092900 \\ \mathrm{O} & 3.70345400 & -0.44283300 & 0.00314300 \\ \mathrm{~N} & 1.76446100 & 0.88219700 & -0.00168300 \\ \mathrm{H} & 2.19929500 & 1.79595700 & -0.00362600 \\ \mathrm{H} & 1.75477700 & -2.29099800 & 0.00275100\end{array}$

8-OH oxidized Guanine (24)

SCF Done: $\mathrm{E}(\mathrm{RB} 3 \mathrm{LYP})=-617.871619027$ a.u.

01

C

$\begin{array}{lll}0.39824700 & 0.62128500 & -0.00275900\end{array}$

$\begin{array}{llll}\mathrm{C} & 0.22859900 & -0.76233500 & -0.00103300\end{array}$

$\begin{array}{llll}\text { C } & -2.00031600 & -0.70125100 & -0.00167500\end{array}$

$\begin{array}{lllll}\text { C } & & -0.75780500 & 1.44072800 & -0.00010700\end{array}$

$\begin{array}{llll}\mathrm{C} & 2.35493400 & -0.20468900 & 0.00078300\end{array}$

$\begin{array}{lllll}\mathrm{N} & & -1.93715700 & 0.67068900 & -0.00012600\end{array}$

$\begin{array}{lllll}\mathrm{N} & & -0.92016400 & -1.46905700 & 0.00193300\end{array}$

$\begin{array}{llll}\mathrm{O} & -0.83681800 & 2.68939200 & 0.00316300\end{array}$

$\mathrm{N} \quad \begin{array}{llll}\mathrm{N} & 1.50201900 & -1.28107600 & 0.00141500\end{array}$

$\mathrm{N} \quad \begin{array}{llll}\mathrm{N} & -3.23523600 & -1.26194900 & -0.05704300\end{array}$

$\mathrm{H} \quad-4.03664900 \quad-0.70119600 \quad 0.20507800$

$\mathrm{H} \quad-3.28987100 \quad-2.24764600 \quad 0.16445900$

$\begin{array}{llll}\mathrm{N} & 1.75517400 & 0.95764800 & -0.00131200\end{array}$

$\mathrm{H} \quad \begin{array}{llll}\mathrm{H} & 1.77210100 & -2.25832300 & 0.00254300\end{array}$

$\begin{array}{llll}\mathrm{O} & 3.67538800 & -0.44752200 & 0.00223700\end{array}$

$\begin{array}{llll}\mathrm{H} & 4.15763600 & 0.39675100 & 0.00209800\end{array}$

$\begin{array}{lllll}\mathrm{H} & -2.80618900 & 1.19923900 & -0.00269800\end{array}$

8- $\mathrm{H}_{2} \mathrm{O}$ oxidized Guanine Cation (25)

SCF Done: $\mathrm{E}(\mathrm{RB} 3 \mathrm{LYP})=-618.226895332$ a.u.

11

C

C

C

C

$\mathrm{C}$

$\mathrm{H}$

$\mathrm{H}$

$\mathrm{N}$

$\mathrm{N}$

$\mathrm{O}$

$\mathrm{N}$

$\mathrm{N}$

$\mathrm{N}$

$\mathrm{H}$

$\mathrm{H}$

$\mathrm{O}$

$\mathrm{H}$

$\mathrm{H}$ $\begin{array}{llll}-0.31660100 & 0.63019500 & -0.13693000\end{array}$

$\begin{array}{lll}-0.16417300 & -0.84003400 & -0.11906800\end{array}$

$\begin{array}{lll}2.05798500 & -0.60857800 & 0.06765700\end{array}$

$\begin{array}{lll}0.93174100 & 1.44363800 & 0.00200100\end{array}$

$\begin{array}{lll}-2.30730000 & -0.21904700 & -0.42064100\end{array}$

$\begin{array}{lll}-2.87297100 & -0.25100300 & -1.35069200\end{array}$

$\begin{array}{lll}-1.66065600 & -2.28087600 & -0.47730100\end{array}$

$\begin{array}{lll}2.08158000 & 0.73582900 & 0.08532100\end{array}$

$\begin{array}{lll}0.95902100 & -1.47060400 & -0.02321300\end{array}$

$\begin{array}{lll}0.88010100 & 2.68428100 & 0.03045600\end{array}$

$\begin{array}{lll}-1.53540900 & 1.00441000 & -0.28189700\end{array}$

$\begin{array}{lll}-1.42071900 & -1.32392800 & -0.23477900\end{array}$

$\begin{array}{lll}3.22036200 & -1.24231900 & 0.15379500\end{array}$

$\begin{array}{lll}4.08124400 & -0.71619800 & 0.23921700\end{array}$

$\begin{array}{lll}3.25033500 & -2.25401400 & 0.14844500\end{array}$

$\begin{array}{lll}-3.38142100 & -0.16016200 & 0.61481700\end{array}$

$\begin{array}{lll}-4.07597300 & -0.84350300 & 0.48286900\end{array}$

$\begin{array}{lll}-3.05517900 & -0.20812000 & 1.54257200\end{array}$
8-OH N7H oxidized Guanine Cation (26)

\begin{tabular}{|c|c|c|c|}
\hline \multicolumn{4}{|c|}{$\begin{array}{l}\text { SCF Done: } E(\text { RB3LYP })=-618.252065906 \text { a.u. } \\
11\end{array}$} \\
\hline $\mathrm{C}$ & 0.30692500 & 0.60506900 & 0.11071400 \\
\hline $\mathrm{C}$ & 0.17693200 & -0.86390800 & 0.09660000 \\
\hline $\mathrm{C}$ & -2.05375200 & -0.60439500 & -0.05502500 \\
\hline $\mathrm{C}$ & -0.92655500 & 1.44607600 & -0.01494700 \\
\hline $\mathrm{C}$ & 2.41754400 & -0.30493500 & 0.33116300 \\
\hline $\mathrm{H}$ & 2.87725100 & -0.30279800 & 1.32381300 \\
\hline $\mathrm{H}$ & 1.66977500 & -2.33316700 & 0.25580900 \\
\hline $\mathrm{N}$ & -2.07132800 & 0.74754400 & -0.08197600 \\
\hline $\mathrm{N}$ & -0.96554100 & -1.47126900 & 0.01723200 \\
\hline $\mathrm{O}$ & -0.82127400 & 2.68113300 & -0.04913300 \\
\hline $\mathrm{N}$ & 1.54296100 & 0.91326400 & 0.23561400 \\
\hline $\mathrm{N}$ & 1.41994400 & -1.35006400 & 0.19375500 \\
\hline $\mathrm{N}$ & -3.22567700 & -1.21651300 & 43300 \\
\hline $\mathrm{H}$ & 909100 & -0.67351300 & -0.16633500 \\
\hline $\mathrm{H}$ & -3.27608400 & -2.22804200 & -0.08692600 \\
\hline $\mathrm{H}$ & 1.92092300 & 1.86475000 & 0.27267200 \\
\hline $\mathrm{O}$ & 3.35185100 & -0.34377800 & -0.68615900 \\
\hline $\mathrm{H}$ & 4.21353400 & -0.05423300 & -0.34207500 \\
\hline
\end{tabular}

\begin{tabular}{lrrr}
\hline \multicolumn{4}{c}{ 8-OH oxidized Guanine (27) } \\
\hline SCF Done: & E(RB3LYP) $=$ & -617.829850696 a.u. \\
$0 ~ 1$ & & & \\
C & 0.36785700 & 0.62475300 & -0.12360800 \\
C & 0.20774200 & -0.84519700 & -0.11593800 \\
C & -2.01518300 & -0.60710200 & 0.05460500 \\
C & -0.87737300 & 1.44130600 & 0.00124500 \\
C & 2.40074500 & -0.22528000 & -0.34462100 \\
$\mathrm{~N}$ & -2.03235600 & 0.74374100 & 0.07626300 \\
N & -0.93555400 & -1.47036500 & -0.03384800 \\
O & -0.82529800 & 2.68630800 & 0.03162900 \\
N & 1.44496400 & -1.33202200 & -0.20597400 \\
N & -3.19711800 & -1.21655200 & 0.13226700 \\
H & -4.04406300 & -0.66998600 & 0.21627800 \\
H & -3.25108500 & -2.22636200 & 0.12671600 \\
N & 1.58937900 & 0.99806200 & -0.22943300 \\
H & 1.70125000 & -2.30989400 & -0.28991900 \\
O & 3.45589500 & -0.27613600 & 0.56898100 \\
H & 3.10749100 & -0.22324600 & 1.47573500 \\
H & 2.85370300 & -0.24281300 & -1.33871600
\end{tabular}




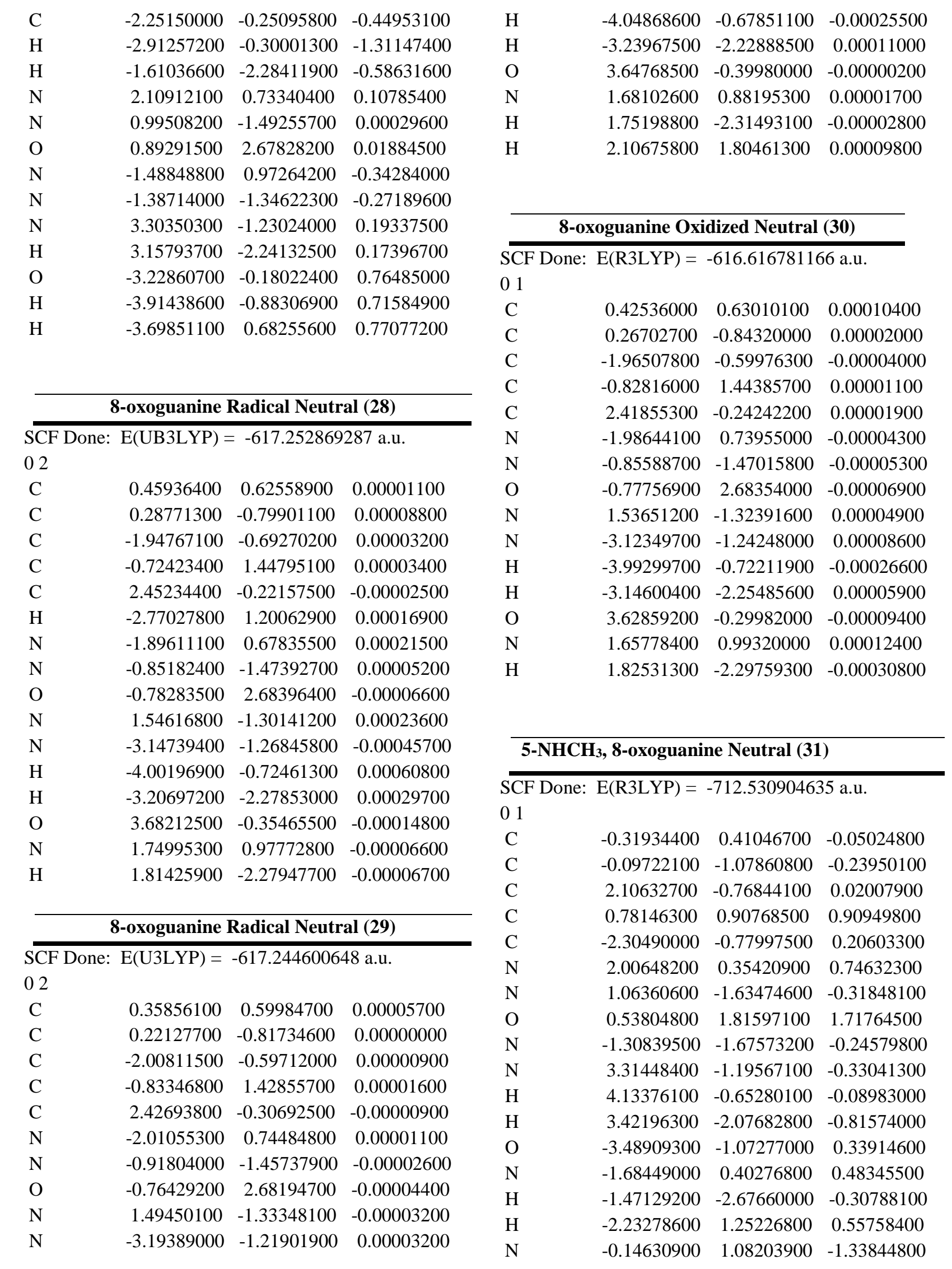




$\begin{array}{lrrr}\mathrm{H} & -0.82236900 & 0.67711400 & -1.98572200 \\ \mathrm{C} & -0.33635200 & 2.54484800 & -1.31290400 \\ \mathrm{H} & 0.49865700 & 3.02830600 & -0.80143400 \\ \mathrm{H} & -0.34357400 & 2.89286800 & -2.34779500 \\ \mathrm{H} & -1.27349200 & 2.86413200 & -0.83773000\end{array}$

$\frac{\text { 5-NHCH3, 8-oxoguanine Anion (31 AN) }}{\text { SCF Done: } \mathrm{E}(\mathrm{R} 3 \mathrm{LYP})=-712.034039288 \text { a.u. }}$

$\begin{array}{lrrr}\mathrm{O} & 3.50488400 & -1.06058200 & -0.39084900 \\ \mathrm{~N} & 1.76233800 & 0.49571200 & -0.42302400 \\ \mathrm{H} & 1.52483900 & -2.63828700 & 0.36775000 \\ \mathrm{~N} & 0.22908400 & 1.10544000 & 1.35393800 \\ \mathrm{C} & 0.26469800 & 2.57784500 & 1.28082700 \\ \mathrm{H} & -0.54403700 & 3.01353100 & 0.67951900 \\ \mathrm{H} & 0.17674100 & 2.96230000 & 2.30056900 \\ \mathrm{H} & 1.22149000 & 2.91113400 & 0.87447700 \\ \mathrm{H} & -0.68231800 & 0.84085600 & 1.72953800\end{array}$

$-11$

$\begin{array}{lrrr}\mathrm{C} & 0.33778200 & 0.42395000 & 0.10304500 \\ \mathrm{C} & 0.08018000 & -1.05258800 & 0.26845100 \\ \mathrm{C} & -2.12762800 & -0.75154700 & -0.01039200 \\ \mathrm{C} & -0.77168300 & 0.96247500 & -0.79491200 \\ \mathrm{C} & 2.27768200 & -0.82995100 & -0.22976800 \\ \mathrm{~N} & -2.01829300 & 0.42102600 & -0.64759900 \\ \mathrm{~N} & -1.08766200 & -1.61077200 & 0.33697200 \\ \mathrm{O} & -0.58436100 & 1.96627500 & -1.51975200 \\ \mathrm{~N} & 1.27412100 & -1.68668100 & 0.26714400 \\ \mathrm{~N} & -3.34808500 & -1.22080400 & 0.25796700 \\ \mathrm{H} & -4.16578200 & -0.69029100 & -0.01461600 \\ \mathrm{H} & -3.45998800 & -2.14069300 & 0.66443800 \\ \mathrm{O} & 3.46093300 & -1.16647500 & -0.35987500 \\ \mathrm{~N} & 1.69349400 & 0.35388800 & -0.55064700 \\ \mathrm{H} & 1.39931500 & -2.69457600 & 0.31007300 \\ \mathrm{H} & 2.28110500 & 1.18210900 & -0.54109500 \\ \mathrm{~N} & 0.29741800 & 1.08439400 & 1.37438500 \\ \mathrm{C} & 0.47841700 & 2.53143500 & 1.26026000 \\ \mathrm{H} & -0.36372500 & 3.07982000 & 0.79699400 \\ \mathrm{H} & 0.58681400 & 2.94068100 & 2.27152600 \\ \mathrm{H} & 1.38423400 & 2.83455300 & 0.70204600\end{array}$

\begin{tabular}{crrr}
\hline \multicolumn{4}{c}{ 5-NHCH3, 8-oxoguanine Anion (31AN') } \\
\cline { 2 - 2 } Done: & E(R3LYP) $=$ & -712.071236811 a.u. \\
-11 & & & \\
$\mathrm{C}$ & 0.39111500 & 0.40795700 & 0.04164600 \\
$\mathrm{C}$ & 0.14662000 & -1.06590600 & 0.28786000 \\
$\mathrm{C}$ & -2.05559600 & -0.79945900 & -0.03306800 \\
$\mathrm{C}$ & -0.70865600 & 0.87504900 & -0.92513500 \\
$\mathrm{C}$ & 2.31605600 & -0.68291800 & -0.20268300 \\
$\mathrm{~N}$ & -1.93996700 & 0.32104000 & -0.77641800 \\
$\mathrm{~N}$ & -1.02867200 & -1.62625900 & 0.39358800 \\
$\mathrm{O}$ & -0.49555100 & 1.79861900 & -1.73858800 \\
$\mathrm{~N}$ & 1.34768200 & -1.63966800 & 0.30793900 \\
$\mathrm{~N}$ & -3.28388200 & -1.23417000 & 0.24684700 \\
$\mathrm{H}$ & -4.09166600 & -0.71772400 & -0.07768200 \\
$\mathrm{H}$ & -3.41121600 & -2.10617100 & 0.74456000
\end{tabular}

\begin{tabular}{lrrr}
\hline \multicolumn{4}{c}{ 5-NHCH3-Sp Anion (32AN) } \\
\cline { 2 - 2 } SCone: & E(R3LYP) $=$ & -712.093130102 & a.u. \\
$-1 ~ 1$ & & & \\
$\mathrm{C}$ & 0.87877900 & 0.77414500 & -0.09330200 \\
$\mathrm{C}$ & 1.88667200 & -1.26420200 & -0.40841800 \\
$\mathrm{~N}$ & 0.55041600 & -1.46979000 & -0.56176400 \\
$\mathrm{H}$ & 0.17264800 & -2.39950800 & -0.41163900 \\
$\mathrm{~N}$ & 2.07931600 & 0.08335100 & -0.16324300 \\
$\mathrm{C}$ & -0.23153400 & -0.28041500 & -0.20906700 \\
$\mathrm{~N}$ & -1.28816900 & 0.03958800 & -1.14409900 \\
$\mathrm{C}$ & -0.98133300 & -0.47361700 & 1.14918700 \\
$\mathrm{O}$ & -0.40293900 & -0.78811200 & 2.21882200 \\
$\mathrm{~N}$ & -2.28446100 & -0.26014200 & 0.95338000 \\
$\mathrm{C}$ & -2.38308300 & 0.03489000 & -0.40426500 \\
$\mathrm{~N}$ & -3.60657200 & 0.34452600 & -0.90082000 \\
$\mathrm{H}$ & -4.41355500 & 0.06898200 & -0.35587900 \\
$\mathrm{H}$ & -3.71963200 & 0.34034400 & -1.90691000 \\
$\mathrm{C}$ & 1.88227000 & 2.87457700 & 0.12992600 \\
$\mathrm{H}$ & 1.58023700 & 3.92269900 & 0.15804500 \\
$\mathrm{H}$ & 2.54521300 & 2.73231300 & -0.73307500 \\
$\mathrm{O}$ & 2.78982500 & -2.11127400 & -0.49593200 \\
$\mathrm{~N}$ & 0.68770700 & 2.02953500 & 0.04442400 \\
$\mathrm{H}$ & 2.46121700 & 2.66200500 & 1.03727600 \\
$\mathrm{H}$ & 3.00049600 & 0.50653100 & -0.15044600
\end{tabular}

\begin{tabular}{lrrr}
\hline \multicolumn{4}{c}{ 5-NHCH3-Sp Anion (32AN') } \\
\hline SCF Done: & E(R3LYP) $=$ & -712.102588649 & a.u. \\
-11 & & & \\
$\mathrm{C}$ & 0.89989800 & 0.71678800 & -0.08777000 \\
$\mathrm{C}$ & 2.02126200 & -1.10413300 & -0.43232500 \\
$\mathrm{~N}$ & 0.70851100 & -1.46612700 & -0.65091900 \\
$\mathrm{H}$ & 0.43862800 & -2.42174300 & -0.43913000 \\
$\mathrm{~N}$ & 2.13968200 & 0.25606500 & -0.17457100 \\
$\mathrm{C}$ & -0.16328000 & -0.37182700 & -0.24699900 \\
$\mathrm{~N}$ & -1.24021700 & -0.04003100 & -1.15625700 \\
$\mathrm{C}$ & -0.89272700 & -0.65424000 & 1.10845500 \\
$\mathrm{O}$ & -0.29677400 & -1.02532000 & 2.14864900 \\
$\mathrm{~N}$ & -2.20002900 & -0.43964700 & 0.94097800
\end{tabular}




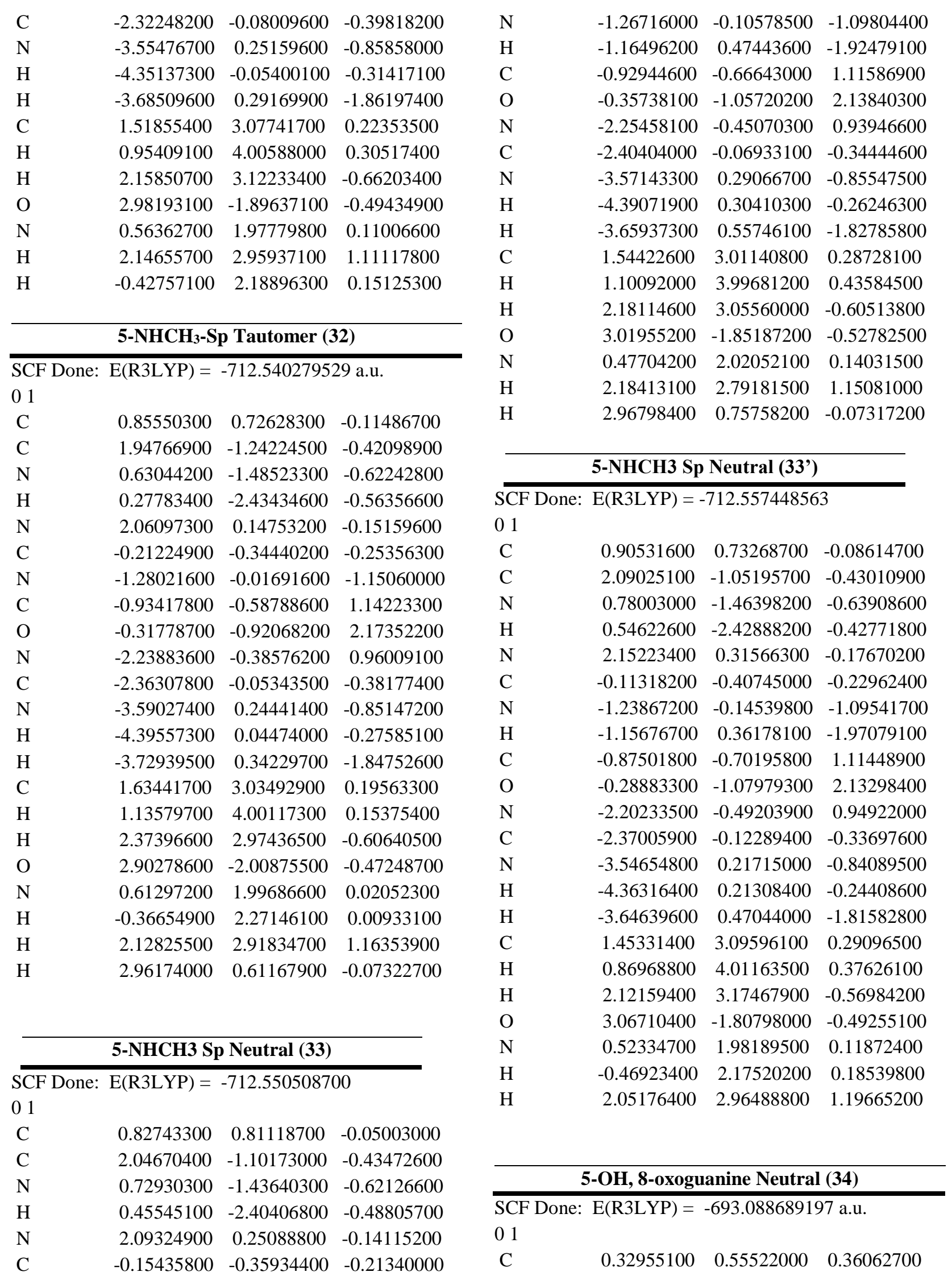




\begin{tabular}{|c|c|c|c|c|c|c|c|}
\hline $\mathrm{C}$ & 0.16664700 & -0.91941500 & 0.01844100 & $\mathrm{~N}$ & 1.94170000 & 0.69783900 & -0.44248400 \\
\hline $\mathrm{C}$ & -2.04866100 & -0.60855100 & -0.14798300 & $\mathrm{~N}$ & 0.95659000 & -1.51347800 & -0.05018100 \\
\hline $\mathrm{C}$ & -0.79988200 & 1.33434900 & -0.33929700 & $\mathrm{O}$ & 0.57208800 & 2.49733000 & -0.73208200 \\
\hline $\mathrm{C}$ & 2.36305300 & -0.39829100 & -0.21171000 & $\mathrm{~N}$ & -1.42117000 & -1.42681600 & -0.07780200 \\
\hline $\mathrm{N}$ & -1.99594900 & 0.70678500 & -0.40797300 & $\mathrm{~N}$ & 3.22141600 & -1.16376700 & -0.04459000 \\
\hline $\mathrm{N}$ & -0.97241900 & -1.50375400 & -0.12341500 & $\mathrm{H}$ & 4.05044000 & -0.59147900 & -0.14490800 \\
\hline $\mathrm{O}$ & -0.60177400 & 2.49763400 & -0.71740400 & $\mathrm{H}$ & 3.31789800 & -2.15862500 & 0.11559600 \\
\hline $\mathrm{N}$ & 1.40530400 & -1.43493900 & -0.13731400 & $\mathrm{O}$ & -3.55464300 & -0.55853700 & -0.47215300 \\
\hline $\mathrm{N}$ & -3.23717200 & -1.17974300 & 0.00212900 & $\mathrm{~N}$ & -1.74886400 & 0.84955200 & -0.01124500 \\
\hline $\mathrm{H}$ & -4.08016300 & -0.62048700 & -0.03288600 & $\mathrm{H}$ & -1.64059100 & -2.37364500 & -0.37297600 \\
\hline $\mathrm{H}$ & -3.30844900 & -2.17950400 & 0.14103200 & $\mathrm{O}$ & -0.14689200 & 0.71700400 & 1.76979800 \\
\hline $\mathrm{O}$ & 3.55926300 & -0.56944500 & -0.40548900 & $\mathrm{H}$ & -0.22594700 & 1.66534100 & 1.97479300 \\
\hline $\mathrm{N}$ & 1.68633300 & 0.78375000 & -0.06910600 & & & & \\
\hline $\mathrm{H}$ & 1.62075800 & -2.39031800 & -0.40776500 & & & & \\
\hline $\mathrm{O}$ & 0.11831000 & 0.81048000 & 1.74157900 & \multicolumn{4}{|c|}{ Spiroiminodihydantion Anion (35AN) } \\
\hline $\mathrm{H}$ & 0.71209600 & 0.25413100 & 2.27540900 & \multirow{2}{*}{\multicolumn{4}{|c|}{$\begin{array}{l}\text { SCF Done: } \mathrm{E}(\mathrm{R} 3 \mathrm{LYP})=-692.666606338 \\
-11\end{array}$}} \\
\hline $\mathrm{H}$ & 2.18243200 & 1.64227700 & 0.14401400 & & & & \\
\hline & & & & $\mathrm{C}$ & 0.77176800 & 1.15179200 & 0.42481400 \\
\hline & & & & $\mathrm{O}$ & 0.31792400 & 2.11880500 & 1.03056900 \\
\hline \multicolumn{4}{|c|}{ 5-OH, 8-oxoguanine Anion (34AN) } & $\mathrm{C}$ & 2.25315700 & -0.29330300 & -0.55000200 \\
\hline $\mathrm{SCl}$ & \multicolumn{3}{|c|}{$\mathrm{E}(\mathrm{R} 3 \mathrm{LYP})=-692.635293394$} & $\mathrm{O}$ & 3.34426000 & -0.80059300 & -0.82897500 \\
\hline-11 & & & & $\mathrm{~N}$ & 1.01725200 & -0.71492500 & -0.92108400 \\
\hline $\mathrm{C}$ & -0.33328600 & 0.57830600 & 0.40510400 & $\mathrm{H}$ & 0.88775800 & -1.68425800 & -1.19299200 \\
\hline $\mathrm{C}$ & -0.17351600 & -0.89713400 & 0.06578100 & $\mathrm{~N}$ & 2.08833900 & 0.86930800 & 0.21044000 \\
\hline $\mathrm{C}$ & 2.04273700 & -0.62060600 & -0.12837800 & $\mathrm{H}$ & 2.86858800 & 1.43829700 & 0.52662100 \\
\hline $\mathrm{C}$ & 0.80132200 & 1.33589700 & -0.28971400 & $\mathrm{C}$ & -0.03815200 & 0.01745000 & -0.22652700 \\
\hline $\mathrm{C}$ & -2.35797900 & -0.38982300 & -0.21090000 & $\mathrm{~N}$ & -1.13144600 & 0.47983400 & -1.04523200 \\
\hline $\mathrm{N}$ & 1.99960700 & 0.70110100 & -0.37529200 & $\mathrm{C}$ & -0.72985600 & -0.84590000 & 0.88337200 \\
\hline $\mathrm{N}$ & 0.96414900 & -1.50323700 & -0.06321900 & $\mathrm{O}$ & -0.09688100 & -1.46801100 & 1.76864400 \\
\hline $\mathrm{O}$ & 0.65829500 & 2.52911500 & -0.63366600 & $\mathrm{~N}$ & -2.04961800 & -0.78732900 & 0.69693000 \\
\hline $\mathrm{N}$ & -1.40686700 & -1.42307500 & -0.08495200 & $\mathrm{C}$ & -2.20656000 & 0.00329700 & -0.43696800 \\
\hline $\mathrm{N}$ & 3.23539100 & -1.20098200 & -0.02537500 & $\mathrm{~N}$ & -3.46492100 & 0.27908800 & -0.85243600 \\
\hline $\mathrm{H}$ & 4.08022100 & -0.64667400 & -0.08806300 & $\mathrm{H}$ & -4.21385600 & -0.30544900 & -0.50448600 \\
\hline $\mathrm{H}$ & 3.30445900 & -2.20246300 & 0.10492700 & $\mathrm{H}$ & -3.58430100 & 0.66793100 & -1.77949900 \\
\hline $\mathrm{O}$ & -3.56325300 & -0.58566600 & -0.38951700 & & & & \\
\hline $\mathrm{N}$ & -1.69662700 & 0.79807400 & -0.13600700 & \multicolumn{4}{|c|}{ Spiroiminodihydantion Anion (35 AN') } \\
\hline $\mathrm{H}$ & -2.21947500 & 1.61708600 & 0.16102700 & \multirow{2}{*}{\multicolumn{4}{|c|}{ Done: $E(R 3 L Y P)=-692.646828663$ a.u. }} \\
\hline $\mathrm{H}$ & -1.61889700 & -2.38776500 & -0.32445600 & & & & \\
\hline \multirow[t]{3}{*}{$\mathrm{O}$} & -0.23148400 & 0.80365100 & 1.75932300 & $\mathrm{C}$ & 0.85183100 & 1.11594500 & 0.24679100 \\
\hline & & & & $\mathrm{O}$ & 0.26533800 & 2.16377300 & 0.78684700 \\
\hline & \multicolumn{3}{|c|}{ 5-OH, 8-oxoguanine Anion (34AN') } & $\mathrm{C}$ & 2.26385700 & -0.32601700 & -0.51381600 \\
\hline \multirow{2}{*}{\multicolumn{4}{|c|}{$\begin{array}{l}\mathrm{SCF} \\
-11\end{array}$}} & $\mathrm{O}$ & 3.35448800 & -0.87467000 & -0.72469000 \\
\hline & & & & $\mathrm{N}$ & 1.03927700 & -0.82890800 & -0.85505500 \\
\hline $\mathrm{C}$ & -0.38985500 & 0.55642500 & 0.34947100 & $\mathrm{H}$ & 0.92418800 & -1.82877100 & -0.98721700 \\
\hline $\mathrm{C}$ & -0.19734000 & -0.92260100 & 0.06856200 & $\mathrm{~N}$ & 2.12857600 & 0.94094200 & 0.10178500 \\
\hline $\mathrm{C}$ & 2.01215900 & -0.61838700 & -0.15088600 & $\mathrm{C}$ & -0.01187800 & -0.02959800 & -0.24274300 \\
\hline $\mathrm{C}$ & 0.74241200 & 1.31909700 & -0.35517300 & $\mathrm{~N}$ & -1.11331600 & 0.34800000 & -1.09680600 \\
\hline $\mathrm{C}$ & -2.35303000 & -0.31641400 & -0.19745700 & $\mathrm{C}$ & -0.70073900 & -0.75886800 & 0.96613600 \\
\hline
\end{tabular}




$\begin{array}{lrrr}\mathrm{O} & -0.06297400 & -1.26966300 & 1.91559500 \\ \mathrm{~N} & -2.02033800 & -0.72907500 & 0.77375500 \\ \mathrm{C} & -2.18330900 & -0.06695500 & -0.43833100 \\ \mathrm{~N} & -3.44451100 & 0.15605100 & -0.87964900 \\ \mathrm{H} & -4.18466200 & -0.40525800 & -0.47816000 \\ \mathrm{H} & -3.56092800 & 0.43270300 & -1.84666900 \\ \mathrm{H} & 0.92020400 & 2.82969600 & 1.07359100\end{array}$

\begin{tabular}{lrrr}
\hline \multicolumn{4}{c}{ Spiroiminodihydantion Tautomer (35) } \\
\hline SCF Done: & E(R3LYP) $=$ & -693.071278512 \\
01 & & & \\
$\mathrm{C}$ & 0.77829700 & 1.12661700 & 0.29288200 \\
$\mathrm{O}$ & 0.32675100 & 2.20548400 & 0.83346500 \\
$\mathrm{C}$ & 2.25635600 & -0.36859900 & -0.53815000 \\
$\mathrm{O}$ & 3.35375200 & -0.86035900 & -0.74391600 \\
$\mathrm{~N}$ & 1.02210000 & -0.77896400 & -0.90442500 \\
$\mathrm{H}$ & 0.86977900 & -1.73905600 & -1.19602700 \\
$\mathrm{~N}$ & 2.05974100 & 0.86887100 & 0.17415600 \\
$\mathrm{H}$ & 2.82704500 & 1.46161300 & 0.48836300 \\
$\mathrm{C}$ & -0.03922600 & -0.00246800 & -0.26418400 \\
$\mathrm{~N}$ & -1.14497600 & 0.41285500 & -1.06583700 \\
$\mathrm{C}$ & -0.70880500 & -0.82701100 & 0.94053700 \\
$\mathrm{O}$ & -0.03740400 & -1.36907000 & 1.83360300 \\
$\mathrm{~N}$ & -2.02369900 & -0.80958900 & 0.73766300 \\
$\mathrm{C}$ & -2.20624800 & -0.07162300 & -0.42126200 \\
$\mathrm{~N}$ & -3.45908200 & 0.13600300 & -0.85457400 \\
$\mathrm{H}$ & -4.23260200 & -0.31887800 & -0.39233100 \\
$\mathrm{H}$ & -3.61768000 & 0.59500400 & -1.74023500 \\
$\mathrm{H}$ & -0.65215400 & 2.24715600 & 0.78718300 \\
& & &
\end{tabular}

$\frac{\text { Spiroiminodihydantion (36) }}{2 \mathrm{CF}}$

\begin{tabular}{|c|c|c|c|}
\hline \multicolumn{4}{|c|}{ SCF Done: $\mathrm{E}(\mathrm{R} 3 \mathrm{LYP})=-693.121945447$} \\
\hline C & 0.75023000 & 1.05890700 & 0.62171200 \\
\hline $\mathrm{O}$ & 0.22638700 & 1.89993600 & 1.33525700 \\
\hline C & 2.31571800 & -0.15764400 & -0.53726700 \\
\hline $\mathrm{O}$ & 3.42292000 & -0.56067600 & -0.87743400 \\
\hline $\mathrm{N}$ & 1.09741200 & -0.56679500 & -1.00075700 \\
\hline $\mathrm{H}$ & 1.01415500 & -1.47412200 & -1.44829900 \\
\hline $\mathrm{N}$ & 2.08065200 & 0.85240900 & 0.40653100 \\
\hline $\mathrm{H}$ & 2.82820100 & 1.39868100 & 0.82414000 \\
\hline $\mathrm{C}$ & 0.01392700 & -0.00592200 & -0.22398400 \\
\hline $\mathrm{N}$ & -1.10817300 & 0.50518500 & -0.96687600 \\
\hline $\mathrm{H}$ & -1.08933400 & 1.42949300 & -1.38807400 \\
\hline $\mathrm{C}$ & -0.71678200 & -1.04467600 & 0.70459100 \\
\hline $\mathrm{O}$ & -0.09842800 & -1.81571900 & 1.44167200 \\
\hline
\end{tabular}

$\begin{array}{lllr}\mathrm{N} & -2.05550400 & -0.93537600 & 0.54195600 \\ \mathrm{C} & -2.24799900 & 0.01701400 & -0.39026100 \\ \mathrm{~N} & -3.44664700 & 0.43824200 & -0.75836400 \\ \mathrm{H} & -4.26942500 & 0.05011000 & -0.31563300 \\ \mathrm{H} & -3.55537600 & 1.14578300 & -1.47426900\end{array}$

\begin{tabular}{lrrr}
\hline \multicolumn{4}{c}{ 5-NHCH3 N7H Guanine Radical Cation (37) } \\
\hline SCF Done: & E(UB3LYP) & -638.297456244 a.u. \\
12 & & & \\
$\mathrm{C}$ & -0.70112700 & -0.08044800 & 0.06894300 \\
$\mathrm{C}$ & 0.10300900 & 1.18193000 & 0.25276400 \\
$\mathrm{C}$ & 2.08227900 & 0.14259800 & 0.12248600 \\
$\mathrm{C}$ & 0.13156800 & -1.00861400 & -0.82008800 \\
$\mathrm{C}$ & -1.88722200 & 1.81588100 & -0.52056500 \\
$\mathrm{~N}$ & 1.48276100 & -0.90023700 & -0.57779300 \\
$\mathrm{~N}$ & 1.41807000 & 1.24543800 & 0.44029600 \\
$\mathrm{O}$ & -0.33331000 & -1.78878100 & -1.65139300 \\
$\mathrm{~N}$ & -0.72867000 & 2.25250700 & -0.01661200 \\
$\mathrm{~N}$ & 3.38260000 & -0.00103300 & 0.44024000 \\
$\mathrm{H}$ & 3.92908800 & -0.75205400 & 0.03828500 \\
$\mathrm{H}$ & 3.87794400 & 0.81555800 & 0.77468500 \\
$\mathrm{~N}$ & -1.89003900 & 0.48654700 & -0.60761400 \\
$\mathrm{H}$ & -0.46711200 & 3.23240800 & 0.04545500 \\
$\mathrm{H}$ & -2.70105200 & 2.46125300 & -0.81782700 \\
$\mathrm{~N}$ & -0.96452900 & -0.75267800 & 1.33750600 \\
$\mathrm{H}$ & -1.36309400 & -0.06465100 & 1.97620300 \\
$\mathrm{C}$ & -1.84414700 & -1.93431100 & 1.26157300 \\
$\mathrm{H}$ & -1.35268300 & -2.73494200 & 0.70496500 \\
$\mathrm{H}$ & -2.01398900 & -2.29079700 & 2.27940100 \\
$\mathrm{H}$ & -2.82097100 & -1.73270900 & 0.80222900 \\
$\mathrm{H}$ & 2.08030400 & -1.58613300 & -1.03441200 \\
$\mathrm{H}$ & -2.70946700 & -0.05370300 & -0.86067500
\end{tabular}

\begin{tabular}{lrrc}
\hline \multicolumn{4}{c}{ 5-NHCH 3 N7H Guanine Radical (38) } \\
\hline SCF Done: & E(UB3LYP) & - & -637.835037644 \\
02 & & & \\
$\mathrm{C}$ & 0.66329300 & -0.09168300 & -0.06353300 \\
$\mathrm{C}$ & -0.11481000 & 1.18064400 & -0.25690400 \\
$\mathrm{C}$ & -2.05520500 & 0.09306600 & -0.06173700 \\
$\mathrm{C}$ & -0.19685500 & -0.98213700 & 0.85661900 \\
$\mathrm{C}$ & 1.91089000 & 1.79827700 & 0.44819600 \\
$\mathrm{~N}$ & -1.52572700 & -0.92338100 & 0.66990500 \\
$\mathrm{~N}$ & -1.43182100 & 1.24395000 & -0.40445100 \\
$\mathrm{O}$ & 0.35597800 & -1.72462800 & 1.71074900 \\
$\mathrm{~N}$ & 0.74007000 & 2.24166100 & -0.03278100 \\
$\mathrm{~N}$ & -3.36039100 & -0.03572400 & -0.43432400 \\
$\mathrm{H}$ & -3.91328700 & -0.73988300 & 0.03529600 \\
$\mathrm{H}$ & -3.84400100 & 0.79952400 & -0.73694700 \\
$\mathrm{~N}$ & 1.88527900 & 0.46546300 & 0.56381100 \\
$\mathrm{H}$ & 0.49044300 & 3.22306400 & -0.10284200 \\
$\mathrm{H}$ & 2.74321600 & 2.43545300 & 0.70622000 \\
$\mathrm{~N}$ & 0.90385300 & -0.78131300 & -1.33729300 \\
$\mathrm{H}$ & 1.38060800 & -0.12213400 & -1.95205100
\end{tabular}




$\begin{array}{llll}\mathrm{C} & 1.69790000 & -2.01922200 & -1.24177700 \\ \mathrm{H} & 1.11929400 & -2.79997200 & -0.74291400 \\ \mathrm{H} & 1.91729500 & -2.36205900 & -2.25534200 \\ \mathrm{H} & 2.65215100 & -1.89890300 & -0.70990300 \\ \mathrm{H} & 2.69634400 & -0.08633000 & 0.81324500\end{array}$

\section{5-NHCH 3 N1H Guanine Radical (39)}

SCF Done: E(UB3LYP) $=-637.836524252$ a.u.

02

$\mathrm{C}$

$\mathrm{C}$

C

C

C

$\mathrm{N}$

$\mathrm{O}$

$\mathrm{N}$

$\mathrm{N}$

$\mathrm{H}$

$\mathrm{H}$

$\mathrm{N}$

$\mathrm{H}$

$\mathrm{H}$

$\mathrm{N}$

$\mathrm{H}$

C

$\mathrm{H}$

$\mathrm{H}$

$\mathrm{H}$

$\mathrm{H}$

$\begin{array}{lll}0.66329300 & -0.09168300 & -0.06353300\end{array}$

$\begin{array}{lll}-0.11481000 & 1.18064400 & -0.25690400\end{array}$

$\begin{array}{lll}-2.05520500 & 0.09306600 & -0.06173700\end{array}$

$\begin{array}{lll}-0.19685500 & -0.98213700 & 0.85661900\end{array}$

$\begin{array}{lll}1.91089000 & 1.79827700 & 0.44819600\end{array}$

$\begin{array}{lll}-1.52572700 & -0.92338100 & 0.66990500\end{array}$

$\begin{array}{lll}-1.43182100 & 1.24395000 & -0.40445100\end{array}$

$\begin{array}{lll}0.35597800 & -1.72462800 & 1.71074900\end{array}$

$\begin{array}{lll}0.74007000 & 2.24166100 & -0.03278100\end{array}$

$\begin{array}{lll}-3.36039100 & -0.03572400 & -0.43432400\end{array}$

$\begin{array}{lll}-3.91328700 & -0.73988300 & 0.03529600\end{array}$

$\begin{array}{lll}-3.84400100 & 0.79952400 & -0.73694700\end{array}$

$\begin{array}{lll}1.88527900 & 0.46546300 & 0.56381100\end{array}$

$\begin{array}{lll}0.49044300 & 3.22306400 & -0.10284200\end{array}$

$\begin{array}{lll}2.74321600 & 2.43545300 & 0.70622000\end{array}$

$\begin{array}{lll}0.90385300 & -0.78131300 & -1.33729300\end{array}$

$\begin{array}{lll}1.38060800 & -0.12213400 & -1.95205100\end{array}$

$1.69790000-2.01922200-1.24177700$

$\begin{array}{lll}1.11929400 & -2.79997200 & -0.74291400\end{array}$

$\begin{array}{lll}1.91729500 & -2.36205900 & -2.25534200\end{array}$

$\begin{array}{lll}2.65215100 & -1.89890300 & -0.70990300\end{array}$

$\begin{array}{lll}2.69634400 & -0.08633000 & 0.81324500\end{array}$
5- $\mathrm{NH}_{2} \mathrm{CH}_{3}$ oxidized Guanine Cation (40)

SCF Done: $\mathrm{E}(\mathrm{RB} 3 \mathrm{LYP})=-637.703587907$ a.u.

11

C

C

C

C

$\mathrm{C}$

$\mathrm{H}$

$\mathrm{H}$

$\mathrm{N}$

$\mathrm{N}$

$\mathrm{O}$

$\mathrm{N}$

$\mathrm{N}$

$\mathrm{N}$

$\mathrm{H}$

$\mathrm{H}$

C

$\mathrm{H}$

$\mathrm{H}$

$\mathrm{H}$

$\begin{array}{llll}\mathrm{N} & -0.86031100 & 1.16553200 & 1.18845700 \\ \mathrm{H} & -1.49389200 & 0.68248000 & 1.83937700 \\ \mathrm{H} & -1.34690800 & 2.02515600 & 0.90076000\end{array}$

5-NHCH3 oxidized Guanine Cation (41)

SCF Done: $\mathrm{E}(\mathrm{RB} 3 \mathrm{LYP})=-637.700130771$ a.u.

11

C

C

C

C

$\mathrm{C}$

$\mathrm{H}$

$\mathrm{H}$

$\mathrm{N}$

$\mathrm{N}$

$\mathrm{O}$

$\mathrm{N}$

$\mathrm{N}$

$\mathrm{N}$

$\mathrm{H}$

$\mathrm{H}$

C

$\mathrm{H}$

$\mathrm{H}$

$\mathrm{H}$

$\mathrm{N}$

$\mathrm{H}$

$\mathrm{H}$
$-0.72990200 \quad 0.38831200$
$\begin{array}{lll}-0.36522700 & -1.07888700 & 0.17490200\end{array}$
$1.79064900 \quad-0.62429900 \quad-0.22695100$
$\begin{array}{llll}0.30623100 & 1.01863000 & -0.93610600\end{array}$
$\begin{array}{lll}-2.52371500 & -0.98720600 & -0.30231900\end{array}$
$\begin{array}{lll}-3.53203100 & -1.32821200 & -0.50230300\end{array}$
$\begin{array}{lll}-1.64115000 & -2.80465000 & 0.25310100\end{array}$
$\begin{array}{lll}1.56071600 & 0.51473600 & -0.88847100\end{array}$
$\begin{array}{lll}0.81857000 & -1.56290600 & 0.17258600\end{array}$
$\begin{array}{llll}-0.03085500 & 1.98207100 & -1.63258400\end{array}$
$\begin{array}{llll}-2.10179100 & 0.23688500 & -0.48759200\end{array}$
$\begin{array}{llll}-1.54879600 & -1.79590000 & 0.15708400\end{array}$
$3.03731400-1.01207100-0.01359700$
$3.80972100-0.42346700-0.30180700$
$3.22519100 \quad-1.90566400 \quad 0.42378600$
$\begin{array}{lll}0.44602000 & 1.50059800 & 1.95521700\end{array}$
$\begin{array}{lll}1.06677100 & 0.63297400 & 2.21172700\end{array}$
$\begin{array}{lll}0.16782400 & 1.99872800 & 2.88590400\end{array}$
$\begin{array}{lll}1.03201400 & 2.20265500 & 1.35992600\end{array}$
$\begin{array}{lll}-0.81170700 & 1.16386000 & 1.26621400\end{array}$
$\begin{array}{lll}-1.40442400 & 0.63960800 & 1.91145700\end{array}$
$\begin{array}{llll}-2.70155300 & 1.01632700 & -0.74709300\end{array}$

SCF Done: $\mathrm{E}(\mathrm{RB} 3 \mathrm{LYP})=-637.258775155$ a.u.

01

C

$\mathrm{C}$

C

C

$\mathrm{C}$

$\mathrm{N}$

$\mathrm{N}$

$\mathrm{O}$

$\mathrm{N}$

$\mathrm{N}$

$\mathrm{H}$

$\mathrm{H}$

$\mathrm{N}$

$\mathrm{H}$

C

$\mathrm{H}$

$\mathrm{H}$

$\mathrm{N}$

$\mathrm{H}$

$\mathrm{H}$

$\mathrm{H}$
$-0.70418700-0.0$
0.01395900
0.01227100
1.23209600
0.17322400
$\begin{array}{lll}1.98102000 & 0.19722800 & 0.02242700\end{array}$
$\begin{array}{lll}0.19127900 & -0.94686300 & -0.91183700\end{array}$
$\begin{array}{llll}-2.10612300 & 1.55107700 & -0.39699500\end{array}$
$\begin{array}{lll}1.52598600 & -0.83478300 & -0.70641000\end{array}$
$\begin{array}{llll}1.29150400 & 1.37590700 & 0.30430100\end{array}$
$\begin{array}{lll}-0.30885100 & -1.73533000 & -1.72842600\end{array}$
$\begin{array}{lll}-0.93464700 & 2.18531500 & 0.04890800\end{array}$
$\begin{array}{lll}3.25074200 & 0.18047800 & 0.41711100\end{array}$
$\begin{array}{lll}3.83450100 & -0.61900600 & 0.20895100\end{array}$
$\begin{array}{lll}3.64558200 & 0.97510800 & 0.90267300\end{array}$
$\begin{array}{llll}-2.04298100 & 0.27213300 & -0.49671000\end{array}$
$\begin{array}{llll}-0.77337200 & 3.18741100 & 0.04201600\end{array}$
$\begin{array}{lll}-1.33896300 & -2.12165300 & 1.29816300\end{array}$
$\begin{array}{lll}-1.52944600 & -2.41828400 & 2.33197700\end{array}$
$\begin{array}{lll}-0.62173800 & -2.82899000 & 0.87504800\end{array}$
$\begin{array}{lll}-0.76200200 & -0.76456900 & 1.32435000\end{array}$
$\begin{array}{lll}-1.34420300 & -0.17912900 & 1.92284300\end{array}$
$\begin{array}{llll}-2.97353200 & 2.15743500 & -0.62949400\end{array}$
$\begin{array}{lll}-2.27896800 & -2.19455200 & 0.73890000\end{array}$ 


\begin{tabular}{lrrl}
\hline \multicolumn{4}{c}{ 5-NHCH3 oxidized Guanine (42') } \\
\hline SCF Done: & E(RB3LYP) & -637.231345318 \\
$0 ~ 1$ & & & \\
$\mathrm{C}$ & 0.65246000 & -0.04112600 & -0.04432600 \\
$\mathrm{C}$ & -0.11945900 & 1.24909800 & 0.17216600 \\
$\mathrm{C}$ & -2.09309300 & 0.12470900 & 0.03938500 \\
$\mathrm{C}$ & -0.22978000 & -0.93803100 & -0.95503100 \\
$\mathrm{C}$ & 1.98815200 & 1.65605000 & -0.38323800 \\
$\mathrm{H}$ & 2.84025400 & 2.28016100 & -0.62473500 \\
$\mathrm{H}$ & 0.62274900 & 3.23643400 & 0.05479900 \\
$\mathrm{~N}$ & -1.53933900 & -0.86954200 & -0.75069300 \\
$\mathrm{~N}$ & -1.39094900 & 1.35413000 & 0.26791600 \\
$\mathrm{O}$ & 0.33735700 & -1.70444300 & -1.77207200 \\
$\mathrm{~N}$ & 1.97256400 & 0.36765400 & -0.50736200 \\
$\mathrm{~N}$ & 0.82383600 & 2.24248600 & 0.08278300 \\
$\mathrm{~N}$ & -3.30174300 & -0.00359500 & 0.47970000 \\
$\mathrm{H}$ & -3.55807300 & 0.84825300 & 0.98146700 \\
$\mathrm{~N}$ & 0.83796000 & -0.76262000 & 1.29381600 \\
$\mathrm{H}$ & 1.46209700 & -0.18201700 & 1.86844300 \\
$\mathrm{C}$ & 1.40058200 & -2.15494400 & 1.22999700 \\
$\mathrm{H}$ & 1.59110000 & -2.46519800 & 2.25728300 \\
$\mathrm{H}$ & 0.66577700 & -2.81132600 & 0.76886800 \\
$\mathrm{H}$ & 2.32825100 & -2.13978700 & 0.66306300 \\
$\mathrm{H}$ & -0.06047500 & -0.80510500 & 1.79055000
\end{tabular}

5, 8-diNHCH Guanine Neutral (43)

SCF Done: $\mathrm{E}(\mathrm{R} 3 \mathrm{LYP})=-733.151728686$

01

C

C

C

C

C

$\mathrm{N}$

$\mathrm{N}$

$\mathrm{O}$

$\mathrm{N}$

$\mathrm{N}$

$\mathrm{H}$

$\mathrm{H}$

$\mathrm{N}$

H

C

$\mathrm{H}$

$\mathrm{H}$

N
C

$\mathrm{H}$

$\mathrm{H}$

$\mathrm{N}$

$\mathrm{H}$

$\mathrm{H}$

$\mathrm{H}$

$\mathrm{H}$

$\mathrm{H}$

$\mathrm{H}$

$$
\begin{array}{rrr}
-4.23522400 & -0.14263100 & 0.57378000 \\
-4.59100800 & 0.25614500 & -0.38830200 \\
-3.96037100 & 0.69797000 & 1.21571900 \\
-3.09563300 & -1.06713900 & 0.43007200 \\
-3.43599000 & -1.87419700 & -0.09471200 \\
-5.05821400 & -0.67511300 & 1.05453400 \\
-0.44075600 & 3.09211600 & -0.84089500 \\
-2.44307800 & -0.10039800 & -1.33336200 \\
1.21388200 & 0.84769700 & -1.79102200 \\
-1.68543100 & 1.48272200 & 0.16872800
\end{array}
$$

\section{5,8-diNHCH3 guanine radical Neutral (44)}

SCF Done: $\mathrm{E}(\mathrm{U} 3 \mathrm{LYP})=-732.493378426$

02

$\begin{array}{llll}\text { C } & 0.08638000 & 0.49588300 & -0.13966700\end{array}$

$\begin{array}{lllll}\text { C } & 0.17105300 & -0.98195300 & -0.46207000\end{array}$

$\begin{array}{llll}\text { C } & 2.31766800 & -1.01563500 & 0.16338300\end{array}$

$\begin{array}{llll}\text { C } & 1.08768400 & 0.77262200 & 1.00455400\end{array}$

$\begin{array}{llll}\text { C } & -2.06111800 & -0.37858900 & -0.42094500\end{array}$

$\mathrm{N}$

$\mathrm{N}$

$\begin{array}{lll}2.23799300 & 0.06680800 & 0.96323000\end{array}$

$\begin{array}{lll}1.27252500 & -1.68508900 & -0.43888200\end{array}$

$\begin{array}{llll}0.85574300 & 1.64991600 & 1.85313700\end{array}$

$\begin{array}{lll}-1.05518700 & -1.40181700 & -0.73922100\end{array}$

$\begin{array}{lll}3.51900300 & -1.57609600 & 0.00946600\end{array}$

$\begin{array}{lll}4.32700300 & -1.17714100 & 0.46798000\end{array}$

$3.62012100 \quad-2.42551300 \quad-0.52928100$

$\begin{array}{lll}-1.31464200 & 0.75612500 & 0.11349400\end{array}$

$\begin{array}{lll}-1.29004800 & -2.36369800 & -0.96540100\end{array}$

$\begin{array}{llll}0.77321200 & 2.70151800 & -1.15476400\end{array}$

$\begin{array}{llll}0.94361100 & 3.12121900 & -2.14886800\end{array}$

$\begin{array}{llll}1.67649700 & 2.86078700 & -0.56207800\end{array}$

$\begin{array}{llll}0.53181900 & 1.25946900 & -1.32964600\end{array}$

$\begin{array}{llll}-0.15564200 & 1.11422000 & -2.06801700\end{array}$

$\begin{array}{lll}-4.29215300 & -0.16290800 & 0.53050800\end{array}$

$\begin{array}{lll}-4.71361100 & -0.08318000 & -0.48230800\end{array}$

$\begin{array}{lll}-4.16732400 & 0.84477300 & 0.93215900\end{array}$

$\begin{array}{lll}-3.01227300 & -0.89304800 & 0.56114100\end{array}$

$\begin{array}{lll}-3.20614100 & -1.85889100 & 0.29734300\end{array}$

$\begin{array}{lll}-5.00724000 & -0.68963400 & 1.16551200\end{array}$

$\begin{array}{lll}-0.05550600 & 3.24646500 & -0.69024800\end{array}$

$\begin{array}{lll}-2.56869300 & -0.05883100 & -1.34496200\end{array}$

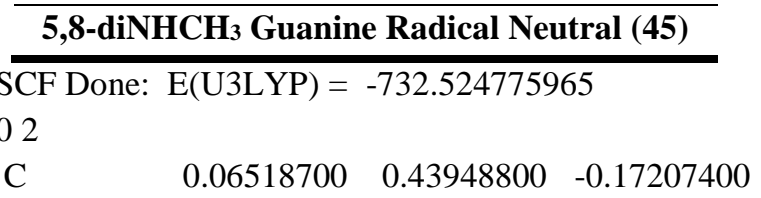




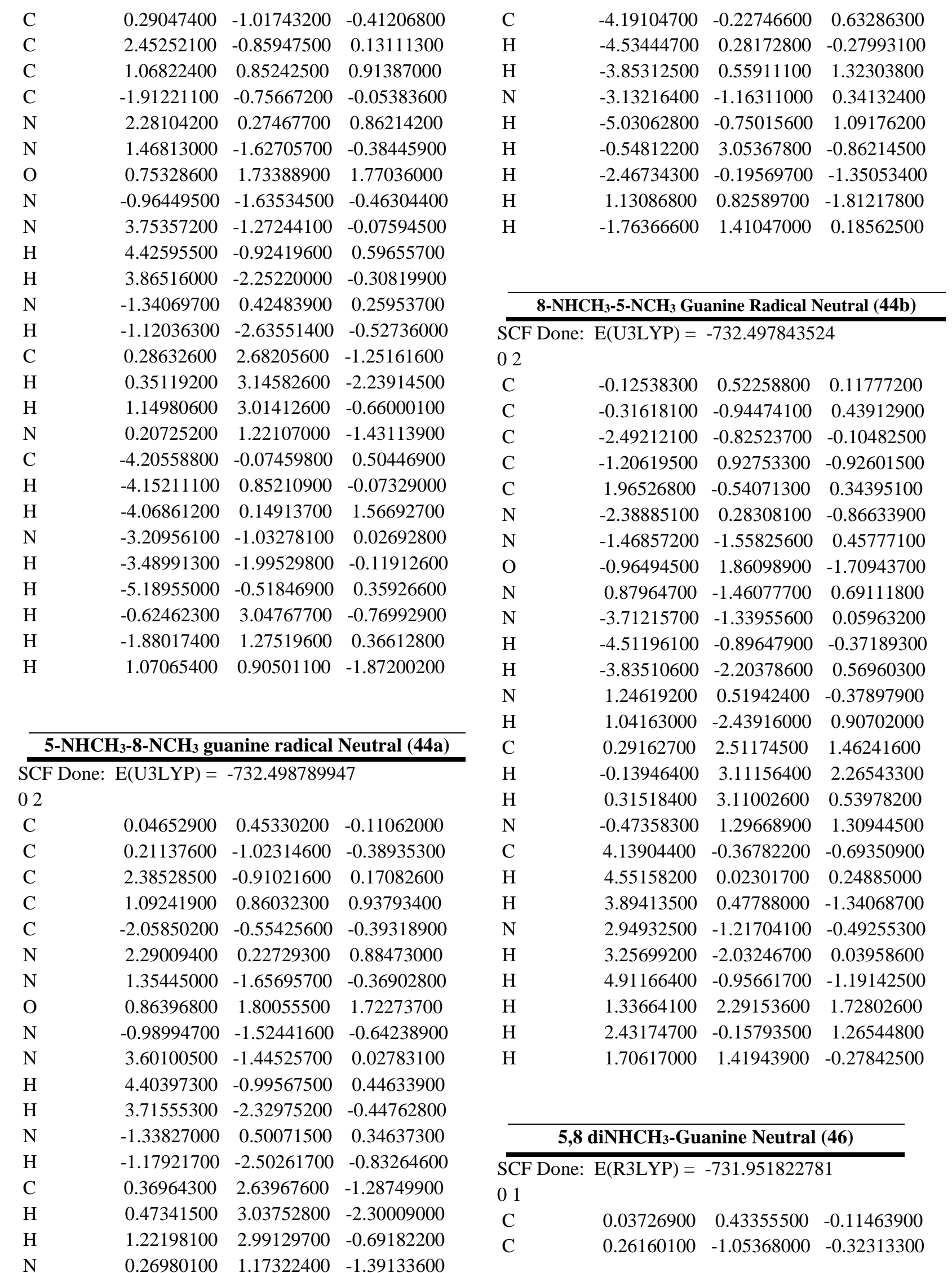




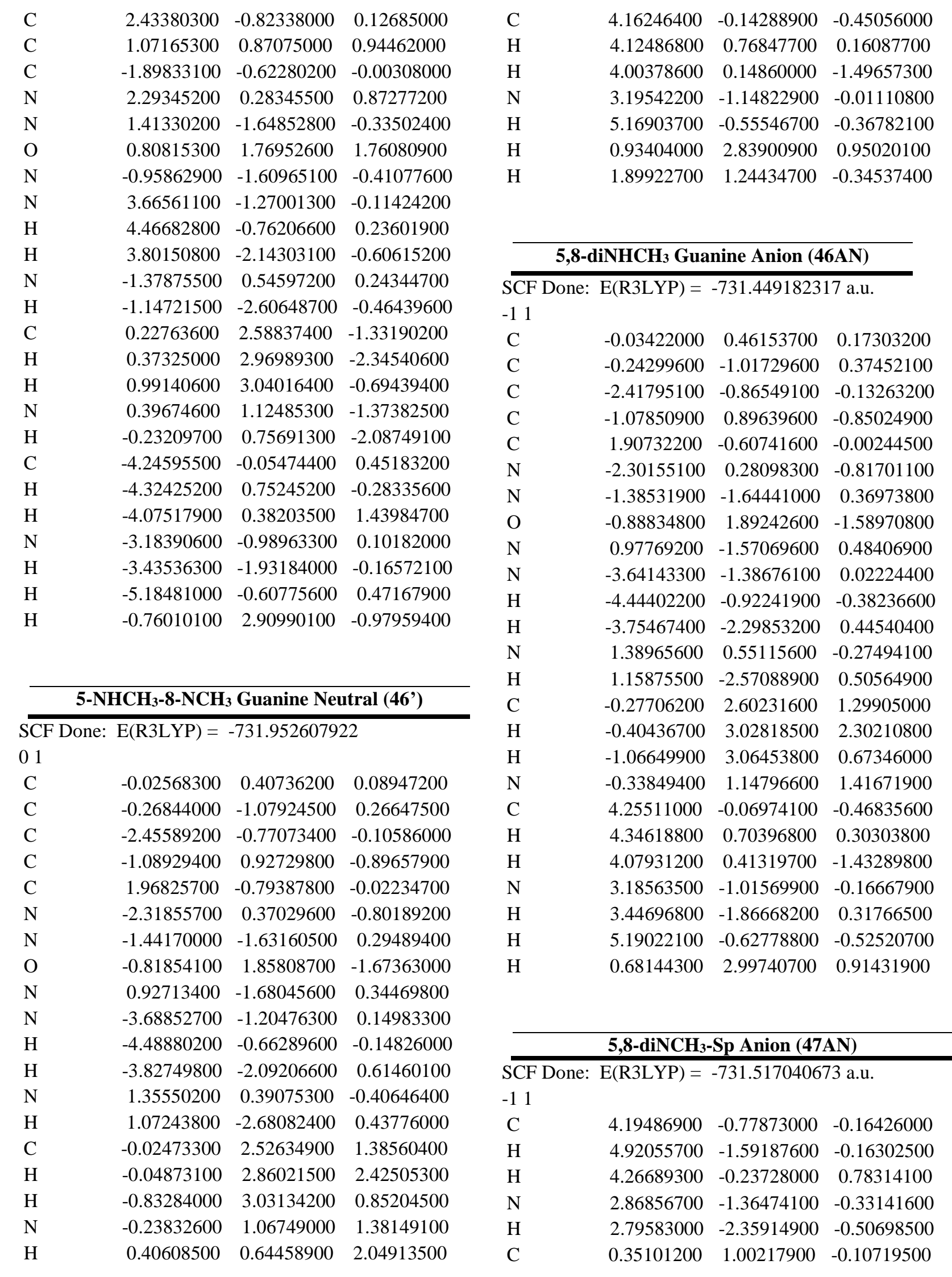




$\begin{array}{lrrr}\mathrm{C} & 1.74191500 & -0.65092100 & -0.32941900 \\ \mathrm{~N} & 0.52330100 & -1.25461000 & -0.54940500 \\ \mathrm{H} & 0.40514500 & -2.20689800 & -0.21407000 \\ \mathrm{~N} & 1.69458100 & 0.67112300 & -0.15445800 \\ \mathrm{C} & -0.51351300 & -0.27483400 & -0.22139300 \\ \mathrm{~N} & -1.61068500 & -0.21252100 & -1.17054500 \\ \mathrm{C} & -1.21457500 & -0.59699400 & 1.12919700 \\ \mathrm{O} & -0.59309000 & -0.79963700 & 2.20496300 \\ \mathrm{~N} & -2.53646400 & -0.62119600 & 0.93771900 \\ \mathrm{C} & -2.68569700 & -0.38654600 & -0.42718700 \\ \mathrm{~N} & -3.95163800 & -0.30810500 & -0.92133500 \\ \mathrm{H} & -4.68073100 & -0.74128400 & -0.36824700 \\ \mathrm{H} & -4.06054900 & -0.37662500 & -1.92594400 \\ \mathrm{C} & 0.77058000 & 3.29117600 & 0.15575600 \\ \mathrm{H} & 0.20831400 & 4.22587100 & 0.21934200 \\ \mathrm{H} & 1.44685900 & 3.36072600 & -0.70550500 \\ \mathrm{~N} & -0.16604200 & 2.17159900 & 0.04001600 \\ \mathrm{H} & 1.39182900 & 3.21292100 & 1.05743000 \\ \mathrm{H} & 4.43168500 & -0.09213900 & -0.98287100\end{array}$

\section{$\frac{\text { 5-NHCH} 3-8-N_{3} H_{3} \text { Sp Anion (47ANa) }}{\text { SCF Done: } \mathrm{E}(\mathrm{R} 3 \mathrm{LYP})=-731.517491016 \text { a.u. }}$} $-11$

C

$\mathrm{H}$

$\mathrm{H}$

$\mathrm{N}$

C

C

$\mathrm{N}$

$\mathrm{H}$

$\mathrm{N}$

C

$\mathrm{N}$

C

$\mathrm{O}$

$\mathrm{N}$

C

$\mathrm{N}$

$\mathrm{H}$

$\mathrm{H}$

C

$\mathrm{H}$

$\mathrm{H}$

$\mathrm{N}$

$\mathrm{H}$

$\mathrm{H}$

$\mathrm{H}$ $\begin{array}{lll}4.18161300 & -0.70194900 & -0.16281700\end{array}$

$\begin{array}{llll}5.00697200 & -1.41776100 & -0.14374900\end{array}$

$\begin{array}{lll}4.19796300 & -0.15257000 & 0.78665800\end{array}$

$\begin{array}{llll}2.92863800 & -1.42871900 & -0.37967100\end{array}$

$\begin{array}{llll}0.41723200 & 0.89035900 & -0.10874700\end{array}$

$\begin{array}{lll}1.84212100 & -0.73421400 & -0.36840900\end{array}$

$\begin{array}{llll}0.58597000 & -1.31726900 & -0.61352700\end{array}$

$\begin{array}{lll}0.46850700 & -2.26023800 & -0.25478000\end{array}$

$\begin{array}{llll}1.71113800 & 0.64394300 & -0.17021200\end{array}$

$-0.44970600 \quad-0.36198300-0.24180000$

$\begin{array}{lll}-1.55734700 & -0.23077100 & -1.17339200\end{array}$

$\begin{array}{lll}-1.14945900 & -0.70335400 & 1.11232000\end{array}$

$\begin{array}{lll}-0.52167300 & -0.94161300 & 2.17497600\end{array}$

$\begin{array}{lll}-2.47259700 & -0.70074300 & 0.92845600\end{array}$

$\begin{array}{lll}-2.62958800 & -0.40778800 & -0.42410400\end{array}$

$\begin{array}{lll}-3.89260700 & -0.37988900 & -0.92656800\end{array}$

$\begin{array}{llll}-4.64710800 & -0.24536500 & -0.26534200\end{array}$

$\begin{array}{llll}-4.01955100 & 0.04347600 & -1.83773600\end{array}$

$\begin{array}{llll}0.64695700 & 3.31568900 & 0.23353200\end{array}$

$\begin{array}{lll}-0.05175900 & 4.13062800 & 0.42215400\end{array}$

$\begin{array}{lll}1.22608100 & 3.54134800 & -0.66734600\end{array}$

$\begin{array}{lll}-0.12461100 & 2.08836400 & 0.06712300\end{array}$

$\begin{array}{lll}1.33385300 & 3.22825300 & 1.08040700\end{array}$

$\begin{array}{lll}4.38978800 & 0.01716300 & -0.96536900\end{array}$

$\begin{array}{lll}-1.13647700 & 2.14299100 & 0.07998900\end{array}$

\begin{tabular}{|c|c|c|c|}
\hline \multicolumn{4}{|c|}{ 5,8-NCH $\mathrm{NC}_{3} \mathrm{Sp}$ anion (47'ANb) } \\
\hline \multicolumn{4}{|c|}{$\begin{array}{l}\text { SCF Done: E( } \\
-11\end{array}$} \\
\hline $\mathrm{C}$ & 4.08956300 & -0.87161500 & -0.15194700 \\
\hline $\mathrm{H}$ & 4.89386500 & -1.60943100 & -0.13243300 \\
\hline $\mathrm{H}$ & 4.10647400 & -0.33418100 & 0.80564600 \\
\hline $\mathrm{N}$ & 2.81680100 & -1.55758700 & -0.38971600 \\
\hline $\mathrm{C}$ & 0.35838600 & 0.97997200 & -0.09771700 \\
\hline $\mathrm{C}$ & 1.76280200 & -0.82292700 & -0.37664800 \\
\hline $\mathrm{N}$ & 0.47463100 & -1.28020900 & -0.63270700 \\
\hline $\mathrm{H}$ & 0.28986000 & -2.23219700 & -0.33137700 \\
\hline $\mathrm{N}$ & 1.66826100 & 0.54617800 & -0.15596000 \\
\hline $\mathrm{C}$ & -0.51701200 & -0.27113200 & -0.24036600 \\
\hline $\mathrm{N}$ & -1.63021800 & -0.13049100 & -1.15963400 \\
\hline $\mathrm{C}$ & -1.20411200 & -0.61766600 & 1.11737500 \\
\hline $\mathrm{O}$ & -0.56564000 & -0.85859100 & 2.17366600 \\
\hline $\mathrm{N}$ & -2.52857800 & -0.62026900 & 0.94530200 \\
\hline $\mathrm{C}$ & -2.69579700 & -0.32343000 & -0.40584100 \\
\hline $\mathrm{N}$ & -3.96162000 & -0.30474600 & -0.90141000 \\
\hline $\mathrm{H}$ & -4.71284400 & -0.17522100 & -0.23537500 \\
\hline $\mathrm{H}$ & -4.09533900 & 0.12269400 & -1.80980000 \\
\hline $\mathrm{C}$ & 0.92997800 & 3.22572500 & 0.19299200 \\
\hline $\mathrm{H}$ & 0.43464100 & 4.18889500 & 0.32707800 \\
\hline $\mathrm{H}$ & 1.56248300 & 3.29118900 & -0.70180500 \\
\hline $\mathrm{N}$ & -0.07962000 & 2.17100300 & 0.06613000 \\
\hline $\mathrm{H}$ & 1.58689200 & 3.05785500 & 1.05661000 \\
\hline $\mathrm{H}$ & 4.32171100 & -0.14823900 & -0.94489100 \\
\hline $\mathrm{H}$ & 2.47693500 & 1.14666000 & -0.05410900 \\
\hline
\end{tabular}

\begin{tabular}{lrrr}
\hline \multicolumn{4}{c}{ 5-NHCH3-8-NCH3 $\mathbf{S p ~ ( 4 7 ' a )}$} \\
\cline { 2 - 2 } Done: & $\mathrm{E}(\mathrm{R} 3 \mathrm{~L}$ ' $)=$ & -731.974058272 & a.u. \\
$0 \mathrm{1}$ & & & \\
$\mathrm{C}$ & 4.23309300 & -0.64691100 & -0.20776300 \\
$\mathrm{H}$ & 5.06462000 & -1.35497200 & -0.18491100 \\
$\mathrm{H}$ & 4.25779000 & -0.07474600 & 0.72727100 \\
$\mathrm{~N}$ & 2.98521700 & -1.38736000 & -0.38999500 \\
$\mathrm{C}$ & 0.44131300 & 0.88221000 & -0.05992100 \\
$\mathrm{C}$ & 1.89318300 & -0.71572800 & -0.34979300 \\
$\mathrm{~N}$ & 0.63847800 & -1.33009300 & -0.55125300 \\
$\mathrm{H}$ & 0.54448200 & -2.27807700 & -0.20206100 \\
$\mathrm{~N}$ & 1.73030400 & 0.65999100 & -0.14743100 \\
$\mathrm{C}$ & -0.39759300 & -0.39626700 & -0.19220200 \\
$\mathrm{~N}$ & -1.51640100 & -0.30998100 & -1.11518300 \\
$\mathrm{C}$ & -1.17442700 & -0.75196900 & 1.12163700 \\
$\mathrm{O}$ & -0.59252600 & -1.02259500 & 2.17894500
\end{tabular}




\begin{tabular}{|c|c|c|c|c|c|c|c|}
\hline $\mathrm{N}$ & -2.51100800 & -0.72649400 & 0.90086700 & 01 & & & \\
\hline $\mathrm{C}$ & -2.66956900 & -0.40362800 & -0.39993000 & $\mathrm{C}$ & 4.17467000 & -0.84991500 & -0.18589900 \\
\hline $\mathrm{N}$ & -3.85937100 & -0.21440100 & -0.95368800 & $\mathrm{H}$ & 4.90570900 & -1.65640200 & -0.19282600 \\
\hline $\mathrm{H}$ & -4.69341500 & -0.30848600 & -0.38952400 & $\mathrm{H}$ & 4.26378600 & -0.29795700 & 0.75331600 \\
\hline $\mathrm{H}$ & -3.95040100 & 0.01008100 & -1.93624800 & $\mathrm{~N}$ & 2.84544600 & -1.45672600 & -0.30485800 \\
\hline $\mathrm{C}$ & 0.64144300 & 3.30225400 & 0.29359900 & $\mathrm{H}$ & 2.77485100 & -2.46053600 & -0.42769500 \\
\hline $\mathrm{H}$ & -0.05928300 & 4.11328200 & 0.48934000 & $\mathrm{C}$ & 0.31213700 & 1.00628400 & -0.10277700 \\
\hline $\mathrm{H}$ & 1.20923800 & 3.53027400 & -0.61307700 & $\mathrm{C}$ & 1.71764100 & -0.77156900 & -0.28335600 \\
\hline $\mathrm{N}$ & -0.12451000 & 2.06989400 & 0.13216400 & $\mathrm{~N}$ & 0.48459300 & -1.28010100 & -0.41342500 \\
\hline $\mathrm{H}$ & 1.33714100 & 3.21764600 & 1.13313900 & $\mathrm{H}$ & 0.29874600 & -2.26889100 & -0.28885500 \\
\hline $\mathrm{H}$ & 4.42248500 & 0.05320400 & -1.03093000 & $\mathrm{~N}$ & 1.64874700 & 0.57712500 & -0.13526000 \\
\hline $\mathrm{H}$ & -1.46296500 & 0.20122800 & -1.99041100 & $\mathrm{C}$ & -0.54997300 & -0.25905800 & -0.17891700 \\
\hline $\mathrm{H}$ & -1.13309300 & 2.12067200 & 0.20373200 & $\mathrm{~N}$ & -1.59704800 & -0.23694300 & -1.16666500 \\
\hline & & & & $\mathrm{C}$ & -1.30356100 & -0.50884200 & 1.17711200 \\
\hline & & & & $\mathrm{O}$ & -0.71709500 & -0.62006000 & 2.27770000 \\
\hline & 5,8-diNCH3 & Sp Neutral (4 & 7’b) & $\mathrm{N}$ & -2.61164600 & -0.57102300 & 0.92003500 \\
\hline & $\mathrm{E}(\mathrm{R} 3 \mathrm{LYP})=$ & -731.9682071 & 37 a.u. & $\mathrm{C}$ & -2.70078200 & -0.40153900 & -0.45660800 \\
\hline 01 & & & & $\mathrm{~N}$ & -3.93031200 & -0.45537700 & -1.02471400 \\
\hline $\mathrm{C}$ & 4.18628000 & -0.68477300 & -0.16900200 & $\mathrm{H}$ & -4.73180000 & -0.33361700 & -0.42137600 \\
\hline $\mathrm{H}$ & 5.01909800 & -1.38988500 & -0.15519900 & $\mathrm{H}$ & -4.02596400 & -0.13240700 & -1.97791100 \\
\hline $\mathrm{H}$ & 4.17746600 & -0.16460600 & 0.79803100 & $\mathrm{C}$ & 0.86050700 & 3.27830400 & 0.05878000 \\
\hline $\mathrm{N}$ & 2.94521800 & -1.41500300 & -0.43024400 & $\mathrm{H}$ & 0.34461000 & 4.23795100 & 0.01265700 \\
\hline $\mathrm{C}$ & 0.33893400 & 0.96355600 & -0.04612200 & $\mathrm{H}$ & 1.57664200 & 3.23877100 & -0.77147300 \\
\hline $\mathrm{C}$ & 1.85879900 & -0.74074300 & -0.38150700 & $\mathrm{~N}$ & -0.12441300 & 2.19756600 & -0.00731400 \\
\hline $\mathrm{N}$ & 0.59332700 & -1.26821800 & -0.64675400 & $\mathrm{H}$ & 1.42555100 & 3.24495300 & 0.99833500 \\
\hline $\mathrm{H}$ & 0.46647200 & -2.24429900 & -0.39963300 & $\mathrm{H}$ & 4.37502900 & -0.18144500 & -1.02755200 \\
\hline $\mathrm{N}$ & 1.67357100 & 0.61218300 & -0.10064700 & $\mathrm{H}$ & 2.45820200 & 1.18642800 & -0.12281400 \\
\hline $\mathrm{C}$ & -0.44225200 & -0.34459100 & -0.22735000 & & & & \\
\hline $\mathrm{N}$ & -1.58388700 & -0.25461800 & -1.11590100 & & & & \\
\hline $\mathrm{C}$ & -1.17030500 & -0.76404300 & 1.09387600 & \multicolumn{4}{|c|}{ 5,8-diNHCH3-Sp Neutral (47) } \\
\hline $\mathrm{O}$ & -0.55180700 & -1.08552300 & 2.11577100 & \multicolumn{4}{|c|}{ SCF Done: $\mathrm{E}(\mathrm{R} 3 \mathrm{LYP})=-731.979241407$} \\
\hline $\mathrm{N}$ & -2.51376000 & -0.72405200 & 0.92317700 & 01 & & & \\
\hline $\mathrm{C}$ & -2.71251000 & -0.35773500 & -0.35938000 & $\mathrm{C}$ & 4.21801600 & -0.69616700 & -0.16774100 \\
\hline $\mathrm{N}$ & -3.91823600 & -0.14130500 & -0.86576100 & $\mathrm{H}$ & 4.97175800 & -1.48262400 & -0.16011800 \\
\hline $\mathrm{H}$ & -4.73248500 & -0.23689100 & -0.27369700 & $\mathrm{H}$ & 4.26901800 & -0.14466300 & 0.77318700 \\
\hline $\mathrm{H}$ & -4.04018000 & 0.11585300 & -1.83691500 & $\mathrm{~N}$ & 2.91206500 & -1.33878800 & -0.32498900 \\
\hline $\mathrm{C}$ & 0.69351700 & 3.24448000 & 0.32308100 & $\mathrm{H}$ & 2.88591500 & -2.33740700 & -0.49379500 \\
\hline $\mathrm{H}$ & 0.10187100 & 4.15250800 & 0.44658300 & $\mathrm{C}$ & 0.37276600 & 0.92493800 & -0.11463500 \\
\hline $\mathrm{H}$ & 1.35953100 & 3.38726400 & -0.53794700 & $\mathrm{C}$ & 1.76201700 & -0.68444600 & -0.32654200 \\
\hline $\mathrm{N}$ & -0.20294900 & 2.10391200 & 0.13725600 & $\mathrm{~N}$ & 0.56313500 & -1.28704300 & -0.53807900 \\
\hline $\mathrm{H}$ & 1.31961400 & 3.12365800 & 1.21642600 & $\mathrm{H}$ & 0.43363100 & -2.27312800 & -0.33463800 \\
\hline $\mathrm{H}$ & 4.39893500 & 0.05811100 & -0.94910600 & $\mathrm{~N}$ & 1.67883900 & 0.66331600 & -0.14765000 \\
\hline $\mathrm{H}$ & 2.43765300 & 1.26725700 & 0.00251000 & $\mathrm{C}$ & -0.49582500 & -0.32375200 & -0.22943800 \\
\hline \multirow[t]{3}{*}{$\mathrm{H}$} & -1.56128100 & 0.32802400 & -1.94668200 & $\mathrm{~N}$ & -1.58126800 & -0.24311300 & -1.17032300 \\
\hline & & & & $\mathrm{C}$ & -1.19825900 & -0.64981300 & 1.14395100 \\
\hline & & & & $\mathrm{O}$ & -0.56719600 & -0.83192200 & 2.20798800 \\
\hline \multicolumn{4}{|c|}{ 5-NCH3, 8-NHCH3-Sp Neutral (47') } & $\mathrm{N}$ & -2.51524100 & -0.69534500 & 0.93223100 \\
\hline \multicolumn{4}{|c|}{ SCF Done: $\mathrm{E}(\mathrm{R} 3 \mathrm{LYP})=-731.962989797$} & $\mathrm{C}$ & -2.65865000 & -0.44487900 & -0.42663100 \\
\hline
\end{tabular}




\begin{tabular}{|c|c|c|c|c|c|c|c|}
\hline $\mathrm{N}$ & -3.90690900 & -0.46116000 & -0.94931600 & $\mathrm{C}$ & 2.16061300 & -0.85029600 & -0.17716900 \\
\hline $\mathrm{H}$ & -4.69022100 & -0.39858900 & -0.31451600 & $\mathrm{~N}$ & -1.99625700 & 0.32150600 & -0.77965600 \\
\hline $\mathrm{H}$ & -4.03940100 & -0.10110600 & -1.88444500 & $\mathrm{~N}$ & -1.18187400 & -1.56364400 & 0.49149200 \\
\hline $\mathrm{C}$ & 0.64603700 & 3.34964500 & 0.17357400 & $\mathrm{O}$ & -0.46446700 & 1.68892100 & -1.80916100 \\
\hline $\mathrm{H}$ & -0.04234600 & 4.17436700 & 0.35315800 & $\mathrm{~N}$ & 1.23752200 & -1.68288300 & 0.32192600 \\
\hline $\mathrm{H}$ & 1.22329400 & 3.55266700 & -0.73246900 & $\mathrm{~N}$ & -3.45894500 & -1.13841800 & 0.28836000 \\
\hline $\mathrm{N}$ & -0.14332600 & 2.12821000 & 0.02300700 & $\mathrm{H}$ & -4.16645100 & -0.77166700 & -0.33552900 \\
\hline $\mathrm{H}$ & 1.33064100 & 3.26014100 & 1.02016800 & $\mathrm{H}$ & -3.59927100 & -2.09646100 & 0.58461600 \\
\hline $\mathrm{H}$ & 4.42229100 & -0.01480200 & -0.99734500 & $\mathrm{~N}$ & 1.65857300 & 0.36372100 & -0.38676900 \\
\hline $\mathrm{H}$ & -1.15470400 & 2.20482400 & 0.01751100 & $\mathrm{H}$ & 1.37546200 & -2.68013500 & 0.45271500 \\
\hline & & & & $\mathrm{C}$ & 0.43979100 & 2.72722100 & 1.13759200 \\
\hline & & & & $\mathrm{H}$ & 0.42742100 & 3.22094400 & 2.11168700 \\
\hline & $\mathrm{HCH}_{3}-8-\mathrm{OH} \mathrm{C}$ & Guanine Neutr & ral (48) & $\mathrm{H}$ & -0.33790500 & 3.18295700 & 0.52105200 \\
\hline & $\mathrm{E}(\mathrm{R} 3 \mathrm{LYP})=$ & -713.71682934 & 46 a.u. & $\mathrm{N}$ & 0.15277700 & 1.29675600 & 1.34161400 \\
\hline 01 & & & & $\mathrm{H}$ & 0.78750300 & 0.92034200 & 2.04575800 \\
\hline $\mathrm{C}$ & -0.25414600 & 0.47349900 & -0.06649500 & $\mathrm{H}$ & 1.41492600 & 2.92432800 & 0.67023200 \\
\hline $\mathrm{C}$ & -0.09333300 & -0.99856200 & -0.38168000 & $\mathrm{O}$ & 3.42192500 & -1.12889200 & -0.43883400 \\
\hline $\mathrm{C}$ & 2.10899700 & -0.86743800 & 0.02751900 & $\mathrm{H}$ & 3.63073800 & -2.07174700 & -0.30893800 \\
\hline $\mathrm{C}$ & 0.85231800 & 0.86006200 & 0.93104400 & $\mathrm{H}$ & 2.22368400 & 1.15231400 & -0.67782200 \\
\hline $\mathrm{C}$ & -2.36870700 & -0.59554100 & -0.11752900 & & & & \\
\hline $\mathrm{N}$ & 2.04443200 & 0.23404900 & 0.79730000 & & & & \\
\hline $\mathrm{N}$ & 1.05342100 & -1.61699600 & -0.45610800 & \multirow{2}{*}{\multicolumn{4}{|c|}{$\begin{array}{c}\text { 5-NHCH } 3-8-\mathrm{OH} \text { Oxidized Guanine Radical } \\
\text { Neutral (50) } \\
\end{array}$}} \\
\hline $\mathrm{O}$ & 0.65193300 & 1.75964600 & 1.76805600 & & & & \\
\hline $\mathrm{N}$ & -1.30645000 & -1.51208600 & -0.55828600 & \multicolumn{4}{|c|}{ SCF Done: $\mathrm{E}(\mathrm{U} 3 \mathrm{LYP})=-713.055428672$ a.u. } \\
\hline $\mathrm{N}$ & 3.31952400 & -1.36582600 & -0.23489800 & 02 & & & \\
\hline $\mathrm{H}$ & 4.14407700 & -0.91314300 & 0.13533400 & $\mathrm{C}$ & 0.31199100 & 0.38957100 & -0.08843200 \\
\hline $\mathrm{H}$ & 3.41270100 & -2.22855400 & -0.75350600 & $\mathrm{C}$ & 0.08277800 & -1.09931200 & 0.07691900 \\
\hline $\mathrm{N}$ & -1.61638100 & 0.49445200 & 0.47702500 & $\mathrm{C}$ & -2.13040500 & -0.78045700 & 0.08369600 \\
\hline $\mathrm{H}$ & -1.49239900 & -2.49328800 & -0.73914900 & $\mathrm{C}$ & -0.90381200 & 0.95975400 & -0.85439600 \\
\hline $\mathrm{C}$ & -0.20128100 & 2.68476800 & -1.20608200 & $\mathrm{C}$ & 2.26980500 & -0.78221900 & -0.57108000 \\
\hline $\mathrm{H}$ & -0.11805800 & 3.10568700 & -2.21061300 & $\mathrm{~N}$ & -2.09803000 & 0.39695800 & -0.57027300 \\
\hline $\mathrm{H}$ & 0.58629300 & 3.12616200 & -0.59200000 & $\mathrm{~N}$ & -1.07855700 & -1.65517600 & 0.27853800 \\
\hline $\mathrm{N}$ & -0.01589100 & 1.22675000 & -1.31208900 & $\mathrm{O}$ & -0.76667900 & 1.92736000 & -1.61985900 \\
\hline $\mathrm{H}$ & -0.67085000 & 0.87190900 & -2.00814000 & $\mathrm{~N}$ & 1.26003000 & -1.70298100 & -0.06568500 \\
\hline $\mathrm{H}$ & -1.17548000 & 2.98472500 & -0.79496600 & $\mathrm{~N}$ & -3.31562700 & -1.21786600 & 0.50798000 \\
\hline $\mathrm{O}$ & -3.18405300 & -1.20099100 & 0.86294100 & $\mathrm{H}$ & -4.14735200 & -0.66469200 & 0.35086100 \\
\hline $\mathrm{H}$ & -3.96956900 & -1.57174000 & 0.43107400 & $\mathrm{H}$ & -3.39315900 & -2.12401700 & 0.95002300 \\
\hline $\mathrm{H}$ & -2.99508400 & -0.30280900 & -0.96550300 & $\mathrm{~N}$ & 1.61283200 & 0.51573200 & -0.70333900 \\
\hline \multirow[t]{3}{*}{$\mathrm{H}$} & -2.07835000 & 1.38868800 & 0.33822000 & $\mathrm{H}$ & 1.38179100 & -2.71050500 & -0.09155500 \\
\hline & & & & $\mathrm{C}$ & 0.30809300 & 2.47594600 & 1.33480100 \\
\hline & & & & $\mathrm{H}$ & 0.46357200 & 2.74562400 & 2.38179100 \\
\hline \multicolumn{4}{|c|}{ 5-NHCH3-8-OH Guanine Radical Neutral (49) } & $\mathrm{H}$ & -0.67429000 & 2.84587200 & 1.03359200 \\
\hline \multirow{2}{*}{\multicolumn{4}{|c|}{$\begin{array}{l}\text { SCF Done: } \mathrm{E}(\mathrm{U} 3 \mathrm{LYP})=-713.069393688 \text { a.u. } \\
02\end{array}$}} & $\mathrm{~N}$ & 0.34363200 & 1.00695300 & 1.25627400 \\
\hline & & & & $\mathrm{H}$ & 1.16908200 & 0.65605100 & 1.74130700 \\
\hline $\mathrm{C}$ & 0.27233500 & 0.45593900 & 0.15033900 & $\mathrm{H}$ & 1.07568100 & 2.97562100 & 0.73434400 \\
\hline $\mathrm{C}$ & 0.01880100 & -0.99548800 & 0.44938400 & $\mathrm{H}$ & 2.62454700 & -1.10459600 & -1.55534800 \\
\hline $\mathrm{C}$ & -2.16416900 & -0.77763900 & 0.00529100 & $\mathrm{O}$ & 3.34927300 & -0.74879100 & 0.34095900 \\
\hline $\mathrm{C}$ & -0.77709600 & 0.86686900 & -0.90186900 & $\mathrm{H}$ & 4.13871800 & -0.43294700 & -0.12731000 \\
\hline
\end{tabular}




\begin{tabular}{lrrl}
\hline & 5-NHCH3-8-OH Guanine Neutral (51) \\
\hline SCF Done: & E(R3LYP) $=$ & -712.502743197 & a.u. \\
01 & & & \\
$\mathrm{C}$ & -0.31842800 & 0.43088400 & -0.06955200 \\
$\mathrm{C}$ & -0.11690100 & -1.06056900 & -0.28532900 \\
$\mathrm{C}$ & 2.07657900 & -0.82858800 & 0.04008900 \\
$\mathrm{C}$ & 0.76410200 & 0.85755800 & 0.94396500 \\
$\mathrm{C}$ & -2.23045100 & -0.61095300 & 0.16008200 \\
$\mathrm{~N}$ & 1.98018200 & 0.27377100 & 0.79743100 \\
$\mathrm{~N}$ & 1.02529500 & -1.65872900 & -0.35291100 \\
$\mathrm{O}$ & 0.53382200 & 1.74142500 & 1.78318700 \\
$\mathrm{~N}$ & -1.35173300 & -1.60799400 & -0.29496200 \\
$\mathrm{~N}$ & 3.28712400 & -1.27378700 & -0.28398900 \\
$\mathrm{H}$ & 4.10977200 & -0.75903600 & 0.00151900 \\
$\mathrm{H}$ & 3.39072000 & -2.14475900 & -0.78787600 \\
$\mathrm{~N}$ & -1.72167600 & 0.54990600 & 0.36770500 \\
$\mathrm{H}$ & -1.57118800 & -2.59942500 & -0.32510700 \\
$\mathrm{C}$ & -0.09078200 & 2.59358700 & -1.26702200 \\
$\mathrm{H}$ & -0.02416400 & 2.97549100 & -2.28833900 \\
$\mathrm{H}$ & 0.77006800 & 2.97514500 & -0.71300200 \\
$\mathrm{~N}$ & -0.03967900 & 1.12203600 & -1.33785900 \\
$\mathrm{H}$ & -0.75128400 & 0.81107000 & -1.99877800 \\
$\mathrm{H}$ & -1.00724000 & 2.98516000 & -0.80922300 \\
$\mathrm{O}$ & -3.49002500 & -1.00496600 & 0.32493300 \\
$\mathrm{H}$ & -4.02836200 & -0.26323400 & 0.65453700 \\
& & &
\end{tabular}

\section{5-OH Guanine Radical (56)}

SCF Done: $\mathrm{E}(\mathrm{UB} 3 \mathrm{LYP})=-618.398040924$ a.u.

02

$\begin{array}{lrrr}\mathrm{C} & 0.71415800 & 0.38755700 & 0.32678800 \\ \mathrm{C} & 0.29551100 & -1.01676300 & 0.04956800 \\ \mathrm{C} & -1.84891900 & -0.40426900 & -0.08462600 \\ \mathrm{C} & -0.30049900 & 1.32845400 & -0.32653700 \\ \mathrm{C} & 2.46180200 & -0.88409400 & -0.45201400 \\ \mathrm{~N} & -1.57210400 & 0.89717500 & -0.36440400 \\ \mathrm{~N} & -0.96061500 & -1.42798100 & -0.02000600 \\ \mathrm{O} & 0.07499800 & 2.45635700 & -0.74324900 \\ \mathrm{~N} & 1.42884900 & -1.72590800 & -0.29462800 \\ \mathrm{~N} & -3.15642100 & -0.72060600 & 0.09236200 \\ \mathrm{H} & -3.85508500 & -0.03702900 & -0.16366600 \\ \mathrm{H} & -3.42387300 & -1.69532200 & 0.09267900 \\ \mathrm{~N} & 2.06459200 & 0.37327100 & -0.20899700 \\ \mathrm{H} & 1.46177600 & -2.71504300 & -0.52147200 \\ \mathrm{H} & 3.45630100 & -1.18149700 & -0.74717100 \\ \mathrm{H} & 2.68159200 & 1.17637300 & -0.22000000 \\ \mathrm{O} & 0.71735800 & 0.62285700 & 1.76013400 \\ \mathrm{H} & 0.77802100 & 1.58183700 & 1.91519500\end{array}$

\section{5-OH Guanine Radical (57)}

SCF Done: $\mathrm{E}(\mathrm{UB} 3 \mathrm{LYP})=-618.402504741$ a.u.

02

$\begin{array}{llll}\mathrm{C} & 0.80743400 & 0.38137800 & 0.31432400\end{array}$

$\begin{array}{llll}\text { C } & 0.35200700 & -1.01041400 & 0.02447200\end{array}$

$\begin{array}{lllll}\mathrm{C} & -1.82078700 & -0.48827000 & -0.05732900\end{array}$

$\begin{array}{lllll}\text { C } & -0.19338500 & 1.33729900 & -0.30098300\end{array}$

$\begin{array}{llll}\text { C } & 2.49132300 & -0.78020100 & -0.48601200\end{array}$

$\begin{array}{llll}\mathrm{N} & -1.48082600 & 0.83365400 & -0.30086700\end{array}$

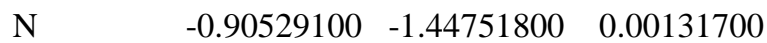

$\begin{array}{lllll}\mathrm{O} & 0.05737300 & 2.47206100 & -0.73891300\end{array}$

$\begin{array}{llll}\mathrm{N} & 1.48194700 & -1.70433100 & -0.33851500\end{array}$

$\mathrm{N} \quad-3.14006200 \quad-0.76769300 \quad 0.12611200$

$\mathrm{H} \quad \begin{array}{llll}-3.82573200 & -0.10250300 & -0.21149000\end{array}$

$\begin{array}{llll}\mathrm{H} & -3.39964200 & -1.74278700 & 0.04082100\end{array}$

$\begin{array}{llll}\mathrm{N} & 2.17630100 & 0.44846600 & -0.18344100\end{array}$

$\mathrm{H} \quad \begin{array}{llll}\mathrm{H} & 1.50661200 & -2.65690000 & -0.68364900\end{array}$

$\begin{array}{lllll}\mathrm{H} & 3.46376900 & -1.09465000 & -0.84333700\end{array}$

$\begin{array}{lllll}\mathrm{O} & 0.77130600 & 0.58473400 & 1.76650700\end{array}$

$\begin{array}{llll}\mathrm{H} & 1.09849800 & 1.48344700 & 1.94756600\end{array}$

$\begin{array}{lllll}\mathrm{H} & -2.21697400 & 1.48223400 & -0.56973700\end{array}$

\begin{tabular}{lrrr}
\hline \multicolumn{4}{c}{ 5-H2O oxidized Guanine Cation (58) } \\
\hline SCF Done: & E(RB3LYP) $=$ & -618.213821116 a.u. \\
11 & & & \\
$\mathrm{C}$ & -0.75384200 & 0.39046300 & 0.17434900 \\
$\mathrm{C}$ & -0.31100400 & -1.05019500 & -0.00799100 \\
$\mathrm{C}$ & 1.83641000 & -0.39911400 & -0.08241200 \\
$\mathrm{C}$ & 0.32348200 & 1.35171800 & -0.37218900 \\
$\mathrm{C}$ & -2.44985200 & -0.78110400 & -0.48116300 \\
$\mathrm{H}$ & -3.43333100 & -1.09758600 & -0.80609900
\end{tabular}




$\begin{array}{rrrr}\mathrm{H} & -1.52125200 & -2.70878900 & -0.53053000 \\ \mathrm{~N} & 1.59275000 & 0.88898700 & -0.36628300 \\ \mathrm{~N} & 0.90541300 & -1.45213900 & -0.05822100 \\ \mathrm{O} & -0.00519700 & 2.49220800 & -0.71424100 \\ \mathrm{~N} & -2.10736700 & 0.45566100 & -0.28821800 \\ \mathrm{~N} & -1.45901900 & -1.72913800 & -0.26821700 \\ \mathrm{~N} & 3.08862400 & -0.78404700 & 0.09727600 \\ \mathrm{H} & 3.83739400 & -0.10262900 & 0.06370200 \\ \mathrm{H} & 3.30564500 & -1.76106900 & 0.25423300 \\ \mathrm{O} & -0.85792200 & 0.61964300 & 1.68293800 \\ \mathrm{H} & -1.28243100 & 1.48531800 & 1.88361600 \\ \mathrm{H} & -0.01504100 & 0.56407800 & 2.18756900\end{array}$

5-HO oxidized Guanine Cation (59)
$\mathrm{H}$

$\begin{array}{lll}0.83510200 & 1.47345700 & 1.93273100\end{array}$

\section{5-OH-8-NHCH ${ }_{3}$ Guanine Neutral (61)}

SCF Done: $\mathrm{E}(\mathrm{R} 3 \mathrm{LYP})=-713.718581875$ a.u.

01

$\mathrm{C}$

C

$\mathrm{C}$

$\mathrm{C}$

$\mathrm{C}$

$\mathrm{N}$

$\mathrm{N}$

5- $\mathrm{H}_{2} \mathrm{O}$ oxidized Guanine neutral (60')

SCF Done: $\mathrm{E}(\mathrm{RB} 3 \mathrm{LYP})=-617.744059287$ a.u.

01

$\begin{array}{llll}\text { C } & -0.68859500 & 0.39564800 & 0.18633100\end{array}$

$\begin{array}{llll}\text { C } & -0.29705000 & -1.04163900 & -0.07178000\end{array}$

$\begin{array}{llll}\mathrm{C} & 1.90470700 & -0.46204300 & -0.09322600\end{array}$

$\begin{array}{lllll}\text { C } & & 0.39271600 & 1.33159500 & -0.39391400\end{array}$

$\begin{array}{llll}\text { C } & -2.45422300 & -0.70317400 & -0.41601800\end{array}$

$\begin{array}{llll}\mathrm{H} & -3.46537200 & -0.96922200 & -0.69864000\end{array}$

$\mathrm{H} \quad-1.59594300 \quad-2.64293600 \quad-0.62241400$

$\mathrm{N} \quad \begin{array}{llll}1.62989400 & 0.85702900 & -0.40758000\end{array}$

$\mathrm{N} \quad \begin{array}{llll}0.89732300 & -1.48088500 & -0.17349300\end{array}$

$\begin{array}{llll}\mathrm{O} & 0.04629700 & 2.48674000 & -0.74706600\end{array}$

$\begin{array}{llll}\mathrm{N} & -2.06763100 & 0.51573200 & -0.16445500\end{array}$

$\mathrm{N} \quad \quad \begin{array}{llll}-1.48961900 & -1.68092900 & -0.31891900\end{array}$

$\mathrm{N} \quad 3.11748500 \quad-0.81668200 \quad 0.17625300$

$\begin{array}{llll}\mathrm{H} & 3.13776700 & -1.82851500 & 0.31613800\end{array}$

$\begin{array}{llll}\mathrm{O} & -0.54196000 & 0.62674600 & 1.71662900\end{array}$

$\begin{array}{llll}\mathrm{H} & -0.70021200 & 1.56901900 & 1.95401000\end{array}$

$\begin{array}{llll}\mathrm{H} & -1.16841800 & 0.08159100 & 2.24340000\end{array}$

$\begin{array}{llll}\text { C } & -0.11511800 & 0.57749000 & 0.40460000\end{array}$

$\begin{array}{llll}-0.26592200 & -0.91132900 & 0.18631500\end{array}$

$\begin{array}{llll}-2.45042800 & -0.63749600 & -0.25577500\end{array}$

$\begin{array}{llll}-1.21244200 & 1.32637600 & -0.36151700\end{array}$

$\begin{array}{llll}2.01074000 & -0.43758700 & 0.30042900\end{array}$

$\begin{array}{llll}-2.38340600 & 0.67940000 & -0.54064800\end{array}$

$\begin{array}{llll}-1.40792700 & -1.51152100 & -0.02011400\end{array}$

$\begin{array}{llll}\mathrm{O} & -1.02710300 & 2.51181200 & -0.70202700\end{array}$

$\begin{array}{llll}\mathrm{N} & 0.94415300 & -1.44853300 & 0.24977100\end{array}$

$\mathrm{N} \quad-3.65990800 \quad-1.20058200 \quad-0.27881800$

$\mathrm{H} \quad-4.47489900 \quad-0.63770000 \quad-0.48145900$

$\mathrm{H} \quad-3.76467900 \quad-2.19080500 \quad-0.10412600$

$\begin{array}{llll}\mathrm{N} & 1.24442800 & 0.79452000 & -0.01132500\end{array}$

$\begin{array}{llll}\mathrm{O} & -0.36093500 & 0.78918200 & 1.81277400\end{array}$

$\begin{array}{llll}\mathrm{H} & -0.41577600 & 1.74705600 & 1.96846000\end{array}$

$\mathrm{N} \quad 3.06535500 \quad-0.76586200 \quad-0.61829300$

$\mathrm{H} \quad 2.71296200 \quad-0.62599100 \quad-1.56519100$

$\begin{array}{llll}\mathrm{C} & 4.26751500 & 0.06319900 & -0.42274600\end{array}$

$\begin{array}{llll}\mathrm{C} & 4.26751500 & 0.06319900 & -0.42274600 \\ \mathrm{H} & 5.02140800 & -0.22633500 & -1.15736100\end{array}$

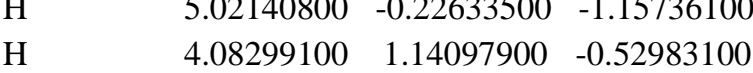

$\begin{array}{llll}\mathrm{H} & 4.67714900 & -0.11949200 & 0.57458100\end{array}$

$\mathrm{H} \quad 1.14234100 \quad-2.43394500 \quad 0.11395200$

$\begin{array}{llll}\mathrm{H} & 2.44417900 & -0.39873500 & 1.30505500\end{array}$

$\begin{array}{llll}\mathrm{H} & 1.65369600 & 1.62114500 & 0.41808000\end{array}$ 


\begin{tabular}{lrrr}
\hline 5-OH-8-NHCH3 Guanine Radical Neutral (62) \\
SCF Done: & E(U3LYP) $=$ & -713.055396092 \\
02 & & & \\
$\mathrm{C}$ & -0.10552400 & 0.56730800 & 0.48537400 \\
$\mathrm{C}$ & -0.25276900 & -0.91637500 & 0.23334100 \\
$\mathrm{C}$ & -2.40621200 & -0.61845300 & -0.30595900 \\
$\mathrm{C}$ & -1.12728500 & 1.32631800 & -0.38284500 \\
$\mathrm{C}$ & 2.01002400 & -0.41824100 & 0.36020600 \\
$\mathrm{~N}$ & -2.29736400 & 0.69295600 & -0.60511600 \\
$\mathrm{~N}$ & -1.38685900 & -1.50793700 & -0.03349000 \\
$\mathrm{O}$ & -0.89889900 & 2.49498300 & -0.74419100 \\
$\mathrm{~N}$ & 0.95612700 & -1.45021400 & 0.31832700 \\
$\mathrm{~N}$ & -3.62578400 & -1.15363900 & -0.36526000 \\
$\mathrm{H}$ & -4.42047300 & -0.57381900 & -0.59947600 \\
$\mathrm{H}$ & -3.75978300 & -2.13942200 & -0.18435200 \\
$\mathrm{~N}$ & 1.30538900 & 0.85877400 & 0.32612100 \\
$\mathrm{H}$ & 1.16659200 & -2.42432200 & 0.12561500 \\
$\mathrm{C}$ & 4.25540000 & -0.00872800 & -0.53080500 \\
$\mathrm{H}$ & 4.72084400 & -0.48032100 & 0.33916100 \\
$\mathrm{H}$ & 4.24702700 & 1.07749200 & -0.37472800 \\
$\mathrm{~N}$ & 2.92510200 & -0.59256100 & -0.74186100 \\
$\mathrm{H}$ & 2.50571300 & -0.23343800 & -1.59900800 \\
$\mathrm{H}$ & 4.87084100 & -0.22267600 & -1.40723700 \\
$\mathrm{O}$ & -0.44920700 & 0.78283700 & 1.86172600 \\
$\mathrm{H}$ & -0.50353500 & 1.74135000 & 2.02040600 \\
$\mathrm{H}$ & 2.57953900 & -0.49002400 & 1.29242200 \\
& & &
\end{tabular}

\begin{tabular}{|c|c|c|c|}
\hline $\mathrm{H}$ & -0.67281500 & 2.03782000 & 1.81959100 \\
\hline $\mathrm{H}$ & 1.44022900 & 2.10262300 & 0.03213500 \\
\hline $\mathrm{N}$ & 3.27213000 & 0.16215200 & -0.22489700 \\
\hline $\mathrm{H}$ & 3.67241500 & 1.08057300 & -0.37359000 \\
\hline $\mathrm{C}$ & 4.13227600 & -1.01548800 & -0.34295100 \\
\hline $\mathrm{H}$ & 5.16357600 & -0.67013400 & -0.39241000 \\
\hline $\mathrm{H}$ & 4.01957500 & -1.66136900 & 0.53201600 \\
\hline $\mathrm{H}$ & 3.90215600 & -1.58445600 & -1.24919800 \\
\hline
\end{tabular}

SCF Done: $\mathrm{E}(\mathrm{R} 3 \mathrm{LYP})=-712.513021707$

01

$\begin{array}{llll}\text { C } & -0.02895500 & 0.51948100 & 0.40259500\end{array}$

$\begin{array}{llll}\text { C } & -0.27658900 & -0.94720800 & 0.10534400\end{array}$

$\begin{array}{llll}\text { C } & -2.45221600 & -0.54329700 & -0.21368500\end{array}$

$\begin{array}{llll}\text { C } & -1.09414000 & 1.34083500 & -0.34588900\end{array}$

$\begin{array}{llll}\text { C } & 1.88221700 & -0.44678000 & -0.05585700\end{array}$

$\mathrm{N} \quad \begin{array}{llll}\mathrm{C} & -2.31587200 & 0.76535300 & -0.47752300\end{array}$

N $\quad-1.43607200 \quad-1.48977600 \quad-0.07840700$

$\begin{array}{llll}\mathrm{O} & -0.85743800 & 2.50812200 & -0.70052500\end{array}$

$\mathrm{N} \quad 0.94125800 \quad-1.50885300 \quad 0.00571200$

$\mathrm{N} \quad \begin{array}{llll}\mathrm{N} & -3.68278800 & -1.04872500 & -0.17441000\end{array}$

$\mathrm{H} \quad-4.48217800 \quad-0.44570600 \quad-0.31883500$

$\mathrm{H} \quad-3.82199300 \quad-2.04136100 \quad-0.03889000$

$\begin{array}{llll}\mathrm{N} & 1.36415600 & 0.74418800 & 0.10065200\end{array}$

$\mathrm{H} \quad 1.12807400 \quad-2.46700300 \quad-0.27611500$

N $\quad 3.16072800 \quad-0.75136100 \quad-0.29232200$

$\mathrm{H} \quad 3.41538200 \quad-1.72936400 \quad-0.33985700$

$\begin{array}{lllll}\mathrm{O} & -0.31183700 & 0.68792800 & 1.80665300\end{array}$

$\mathrm{H} \quad-0.12502700 \quad 1.61570900 \quad 2.02851400$

$\begin{array}{lllll}\text { C } & 4.22247100 & 0.24919400 & -0.31240800\end{array}$

$\begin{array}{lllll}\mathrm{H} & 4.36601400 & 0.69747400 & 0.67576200\end{array}$

$\mathrm{H} \quad 5.14400400 \quad-0.24718400 \quad-0.61532300$

$\mathrm{H} \quad 3.99332800 \quad 1.03989900 \quad-1.03078700$

\begin{tabular}{lrrr}
\hline \multicolumn{4}{c}{ 5-OH-8-NCH3 Guanine Neutral (64') } \\
\cline { 2 - 2 } SCF Done: & $\mathrm{E}(\mathrm{R} 3 \mathrm{LYP})=$ & -712.509910521 \\
01 & & & \\
$\mathrm{C}$ & -0.15450600 & 0.72385100 & 0.30865400 \\
$\mathrm{C}$ & -0.01827900 & -0.76702600 & 0.08127800 \\
$\mathrm{C}$ & -2.24552100 & -0.94252100 & -0.13271500 \\
$\mathrm{C}$ & -1.44040300 & 1.22283600 & -0.36587200 \\
$\mathrm{C}$ & 2.04238400 & 0.16272600 & -0.14233900 \\
$\mathrm{~N}$ & -2.47217900 & 0.35024600 & -0.41580500 \\
$\mathrm{~N}$ & -1.01253700 & -1.58490300 & -0.03333000 \\
$\mathrm{O}$ & -1.52192100 & 2.40540500 & -0.73953600
\end{tabular}




\begin{tabular}{lrrr}
$\mathrm{N}$ & 1.29629200 & -1.03524300 & -0.00991800 \\
$\mathrm{~N}$ & -3.29515600 & -1.75151100 & -0.02215800 \\
$\mathrm{H}$ & -4.23214400 & -1.38083000 & -0.11238000 \\
$\mathrm{H}$ & -3.16077400 & -2.73929200 & 0.15022500 \\
$\mathrm{~N}$ & 1.10459500 & 1.19731500 & -0.20668500 \\
$\mathrm{O}$ & -0.30892100 & 0.90244000 & 1.72233900 \\
$\mathrm{H}$ & -0.55571500 & 1.82828500 & 1.88960400 \\
$\mathrm{~N}$ & 3.30523100 & 0.30215600 & -0.24167600 \\
$\mathrm{C}$ & 4.13420100 & -0.90362900 & -0.18945700 \\
$\mathrm{H}$ & 5.18447700 & -0.60980900 & -0.18465600 \\
$\mathrm{H}$ & 3.94829300 & -1.50143300 & 0.71103000 \\
$\mathrm{H}$ & 3.97258800 & -1.54510400 & -1.06461700 \\
$\mathrm{H}$ & 1.68260700 & -1.95715200 & -0.18473200 \\
$\mathrm{H}$ & 1.41641500 & 2.11873900 & 0.08280400 \\
\multicolumn{4}{c}{} \\
\multicolumn{4}{c}{} \\
\hline 5CF Done: E(R3LYP) $=$ & -712.053511377
\end{tabular}

$\begin{array}{lrrr}\mathrm{C} & -1.44199200 & 1.23773700 & -0.30024000 \\ \mathrm{C} & 2.03241700 & 0.18465400 & -0.15241100 \\ \mathrm{~N} & -2.48057800 & 0.36524000 & -0.36407000 \\ \mathrm{~N} & -1.01289300 & -1.57299400 & -0.01745900 \\ \mathrm{O} & -1.56191100 & 2.43128200 & -0.65883900 \\ \mathrm{~N} & 1.28977700 & -1.01159800 & -0.02035800 \\ \mathrm{~N} & -3.29653800 & -1.75226500 & -0.04014100 \\ \mathrm{H} & -4.23334700 & -1.38374500 & -0.14709400 \\ \mathrm{H} & -3.16158000 & -2.74664900 & 0.09093200 \\ \mathrm{~N} & 1.10855700 & 1.22725200 & -0.21998200 \\ \mathrm{O} & -0.27831400 & 0.98019700 & 1.73092500 \\ \mathrm{~N} & 3.30269800 & 0.30589900 & -0.24475800 \\ \mathrm{C} & 4.10576200 & -0.91865100 & -0.17546400 \\ \mathrm{H} & 5.16269700 & -0.64711500 & -0.16784700 \\ \mathrm{H} & 3.90073800 & -1.50251300 & 0.73034900 \\ \mathrm{H} & 3.93428900 & -1.56793100 & -1.04326800 \\ \mathrm{H} & 1.68426300 & -1.93626500 & -0.16409800 \\ \mathrm{H} & 1.44022500 & 2.10731900 & 0.16642200\end{array}$

$-11$

$\begin{array}{lrrr}\mathrm{C} & -0.04516200 & 0.54175700 & 0.48460900 \\ \mathrm{C} & -0.27233800 & -0.92104800 & 0.14132500 \\ \mathrm{C} & -2.44750600 & -0.54645400 & -0.21026400 \\ \mathrm{C} & -1.09454700 & 1.34656600 & -0.28747300 \\ \mathrm{C} & 1.87841400 & -0.41965900 & -0.05034300 \\ \mathrm{~N} & -2.31634300 & 0.77111800 & -0.45605300 \\ \mathrm{~N} & -1.43340400 & -1.48172300 & -0.04626700 \\ \mathrm{O} & -0.88066900 & 2.53250100 & -0.62815100 \\ \mathrm{~N} & 0.94026600 & -1.48535500 & 0.03960300 \\ \mathrm{~N} & -3.68041400 & -1.05571100 & -0.21570400 \\ \mathrm{H} & -4.47488500 & -0.45357200 & -0.39126600 \\ \mathrm{H} & -3.81926700 & -2.05221500 & -0.10714800 \\ \mathrm{~N} & 1.37061900 & 0.76651200 & 0.12328800 \\ \mathrm{H} & 1.13106300 & -2.44173700 & -0.24704900 \\ \mathrm{~N} & 3.15026400 & -0.74329300 & -0.34301500 \\ \mathrm{H} & 3.39964600 & -1.72263700 & -0.27626800 \\ \mathrm{O} & -0.30266400 & 0.76318300 & 1.83795900 \\ \mathrm{C} & 4.22769100 & 0.24002900 & -0.31486100 \\ \mathrm{H} & 4.37945900 & 0.64702100 & 0.69133400 \\ \mathrm{H} & 5.14318700 & -0.25470800 & -0.64018500 \\ \mathrm{H} & 4.01124400 & 1.06439800 & -0.99880100\end{array}$

\section{5-O-8- $\mathrm{NCH}_{3}$ Oxidized Guanine Anion (64'aAN)}

SCF Done: $\mathrm{E}(\mathrm{R} 3 \mathrm{LYP})=-712.054851182$

$-11$

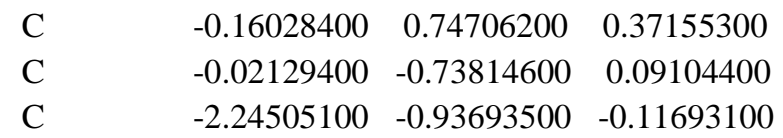

\section{5-OH-8- $\mathrm{NCH}_{3}$ Oxidized Guanine Anion (64'bAN)}

SCF Done: $\mathrm{E}(\mathrm{R} 3 \mathrm{LYP})=-712.043629635$

$-11$

$\begin{array}{llll}\text { C } & -0.11285500 & 0.74807600 & 0.32160700\end{array}$

$\begin{array}{lllll}\text { C } & 0.01592100 & -0.74954400 & 0.15348000\end{array}$

$\begin{array}{lllll}\text { C } & -2.19540800 & -0.94763200 & -0.15378300\end{array}$

$\begin{array}{lllll}\text { C } & -1.37362400 & 1.21639300 & -0.41017000\end{array}$

$\begin{array}{llll}\text { C } & 2.02297200 & 0.25783300 & -0.07795500\end{array}$

$\begin{array}{llll}\mathrm{N} & -2.40305900 & 0.34077000 & -0.49852900\end{array}$

$\begin{array}{lllll}\mathrm{N} & & -0.98068600 & -1.58427400 & 0.04019400\end{array}$

$\begin{array}{llll}\mathrm{O} & -1.47012300 & 2.39357200 & -0.82262300\end{array}$

$\mathrm{N} \quad 1.32182800 \quad-1.00127300 \quad 0.09227200$

$\mathrm{N} \quad \begin{array}{llll}\mathrm{N} & -3.26427600 & -1.74037200 & -0.08004300\end{array}$

$\mathrm{H} \quad-4.18852300 \quad-1.36068100 \quad-0.24211800$

$\mathrm{H} \quad \begin{array}{llll}\mathrm{H} & -3.15719500 & -2.72284400 & 0.13762300\end{array}$

$\mathrm{N} \quad \begin{array}{llll}1.14471800 & 1.28921500 & -0.04707200\end{array}$

$\begin{array}{lllll}\mathrm{O} & -0.43637500 & 0.93536500 & 1.74957100\end{array}$

$\begin{array}{llll}\mathrm{H} & -0.47261000 & 1.89670400 & 1.89398200\end{array}$

$\begin{array}{llll}\mathrm{N} & 3.30586800 & 0.33834800 & -0.26796100\end{array}$

C $\quad 4.07202900 \quad-0.90807100 \quad-0.30650800$

$\mathrm{H} \quad 5.12413400 \quad-0.67147200 \quad-0.48273200$

$\mathrm{H} \quad 4.02133700 \quad-1.47292800 \quad 0.63480900$

$\mathrm{H} \quad 3.75420300 \quad-1.58225100 \quad-1.11487000$

$\mathrm{H} \quad \begin{array}{llll}1.72567500 & -1.91724800 & -0.07432800\end{array}$

5-OH-8-NHCH ${ }_{3}$-Sp Neutral (65)

SCF Done: $\mathrm{E}(\mathrm{R} 3 \mathrm{LYP})=-712.519420460$ 
01

\section{C}

$\mathrm{H}$

$\mathrm{H}$

$\mathrm{N}$

$\mathrm{H}$

$\mathrm{C}$

$\mathrm{O}$

C

$\mathrm{N}$

$\mathrm{H}$

$\mathrm{N}$

C

$\mathrm{N}$

C

$\mathrm{O}$

$\mathrm{N}$

C

$\mathrm{N}$

$\mathrm{H}$

$\mathrm{H}$

$\mathrm{H}$

$\mathrm{H}$ $\begin{array}{llll}4.28089700 & -0.11679300 & -0.15947400\end{array}$

$\begin{array}{llll}4.50407300 & 0.78652400 & -0.73203400\end{array}$

$\begin{array}{llll}5.06957300 & -0.84922700 & -0.32573300\end{array}$

$3.02068500-0.72008900-0.60365900$

$3.06313900-1.59273300 \quad-1.11932000$

$\begin{array}{llll}0.37403200 & 1.16756600 & 0.32583700\end{array}$

$\begin{array}{lll}-0.16545900 & 2.23181400 & 0.87309700\end{array}$

$\begin{array}{llll}1.83427300 & -0.18096700 & -0.41693000\end{array}$

$\begin{array}{llll}0.67371200 & -0.71074500 & -0.86100100\end{array}$

$\begin{array}{llll}0.59098900 & -1.69652500 & -1.09006400\end{array}$

$\begin{array}{llll}1.66694100 & 1.02145900 & 0.24366900\end{array}$

$\begin{array}{llll}-0.43298300 & 0.01955100 & -0.24010600\end{array}$

$\begin{array}{llll}-1.53237300 & 0.40278300 & -1.07504400\end{array}$

$\begin{array}{llll}-1.10221000 & -0.83354400 & 0.92273700\end{array}$

$\begin{array}{llll}-0.44401100 & -1.36734800 & 1.83668900\end{array}$

$\begin{array}{llll}-2.41567900 & -0.85733600 & 0.69671400\end{array}$

$\begin{array}{llll}-2.59180300 & -0.11623200 & -0.46254400\end{array}$

$\begin{array}{llll}-3.84704000 & 0.07115000 & -0.91440700\end{array}$

$\begin{array}{llll}-4.59924800 & -0.46809500 & -0.51095000\end{array}$

$\begin{array}{llll}-3.98764300 & 0.48252800 & -1.82634500\end{array}$

$\begin{array}{llll}4.23722700 & 0.12493400 & 0.90333800\end{array}$

$\begin{array}{lll}-1.13929500 & 2.20882600 & 0.80179000\end{array}$

\section{5-8-diOH guanine Radical Neutral (68)}

\begin{tabular}{lrrr}
\hline SCF Done: & $\mathrm{E}(\mathrm{U} 3 \mathrm{LYP})=$ & -693.632216958 \\
$0 \mathrm{2}$ & & & \\
$\mathrm{C}$ & 0.28269400 & 0.57542400 & 0.41028200 \\
$\mathrm{C}$ & 0.11065500 & -0.88988000 & 0.21403400 \\
$\mathrm{C}$ & -2.08379100 & -0.65523800 & -0.14209900 \\
$\mathrm{C}$ & -0.81487800 & 1.29298800 & -0.37507900 \\
$\mathrm{C}$ & 2.25222000 & -0.43078400 & -0.20342500 \\
$\mathrm{~N}$ & -1.99514800 & 0.66159300 & -0.47263000 \\
$\mathrm{~N}$ & -1.05123300 & -1.50578900 & 0.05403600 \\
$\mathrm{O}$ & -0.58677000 & 2.44256100 & -0.84844700 \\
$\mathrm{~N}$ & 1.37763700 & -1.43282700 & -0.03416100 \\
$\mathrm{~N}$ & -3.34462400 & -1.17279200 & -0.03280000 \\
$\mathrm{H}$ & -4.09486600 & -0.64145900 & -0.45432400 \\
$\mathrm{H}$ & -3.43557700 & -2.17999700 & -0.06234100 \\
$\mathrm{~N}$ & 1.65345700 & 0.75581500 & -0.06094400 \\
$\mathrm{H}$ & 1.58401600 & -2.40972100 & -0.22086300 \\
$\mathrm{O}$ & 0.17091200 & 0.91530700 & 1.81428600 \\
$\mathrm{H}$ & 0.05307800 & 1.87867600 & 1.88872500 \\
$\mathrm{O}$ & 3.53525000 & -0.52514100 & -0.47457500 \\
$\mathrm{H}$ & 3.82323400 & -1.44461900 & -0.62406400 \\
$\mathrm{H}$ & 2.15295300 & 1.63824200 & -0.05402700
\end{tabular}

\section{5,8-diOH Guanine Neutral (67)}

\section{SCF Done: E(R3LYP $)=-694.278042245$}

01

C

\section{C}

C

C

C

$\mathrm{N}$

$\mathrm{N}$

$\mathrm{O}$

$\mathrm{N}$

$\mathrm{N}$

$\mathrm{H}$

$\mathrm{H}$

$\mathrm{N}$

$\mathrm{H}$

$\mathrm{O}$

$\mathrm{H}$

$\mathrm{O}$

$\mathrm{H}$

$\mathrm{H}$

$\mathrm{H}$

$\begin{array}{rrr}0.26351500 & 0.60414700 & 0.36474000 \\ 0.14274300 & -0.89096200 & 0.17422300 \\ -2.06523700 & -0.68105500 & -0.17923300 \\ -0.88270100 & 1.30968400 & -0.37309000 \\ 2.39999900 & -0.34513400 & 0.12551100 \\ -2.04350100 & 0.62972200 & -0.49351600 \\ -0.98802700 & -1.52310000 & 0.02797300 \\ -0.74242700 & 2.49211000 & -0.74033500 \\ 1.37127100 & -1.39318000 & 0.18891900 \\ -3.25618100 & -1.27995700 & -0.14040700 \\ -4.09825700 & -0.74740900 & -0.31189400 \\ -3.32189900 & -2.26966600 & 0.05541200 \\ 1.60393600 & 0.85202800 & -0.10415100 \\ 1.59579800 & -2.36965500 & 0.02679800 \\ 0.07120300 & 0.84229800 & 1.77211000 \\ -0.01215700 & 1.80137200 & 1.90902200 \\ 3.26964900 & -0.55794100 & -0.96128400 \\ 4.08621200 & -0.96388700 & -0.63189300 \\ 2.02081600 & 1.68151800 & 0.31145400 \\ 2.97968100 & -0.32268600 & 1.05254400\end{array}$

\section{5-8-diOH Guanine Radical Neutral (69)}

SCF Done: $\mathrm{E}(\mathrm{U} 3 \mathrm{LYP})=-693.611350170$

02

$\begin{array}{llll}\text { C } & 0.28651300 & 0.59190000 & 0.38310900\end{array}$

C $\quad 0.15050000 \quad-0.89745400 \quad 0.15990900$

C $\quad-2.04966800 \quad-0.65354100 \quad-0.18281100$

$\begin{array}{lllll}\text { C } & -0.83323800 & 1.32171600 & -0.38002900\end{array}$

$\begin{array}{llll}\mathrm{C} & 2.39681100 & -0.35330700 & 0.13502200\end{array}$

$\mathrm{N} \quad-2.00243300 \quad 0.65799400 \quad-0.49402500$

$\mathrm{N} \quad \quad-0.98467200 \quad-1.51610600 \quad 0.00332900$

O $\quad-0.66736600 \quad 2.49554000 \quad-0.75431600$

$\mathrm{N} \quad \begin{array}{llll}1.37832900 & -1.40276400 & 0.13742900\end{array}$

$\mathrm{N} \quad-3.25104000 \quad-1.22654000 \quad-0.13289400$

$\mathrm{H} \quad-4.08361400 \quad-0.67582400 \quad-0.29483300$

$\mathrm{H} \quad-3.33586400 \quad-2.21622500 \quad 0.05677900$

$\begin{array}{llll}\mathrm{N} & 1.66352200 & 0.90721800 & 0.04356700\end{array}$

$\mathrm{H} \quad 1.60405100-2.37884000 \quad-0.02837600$

$\begin{array}{llll}\mathrm{O} & 0.10507400 & 0.82011900 & 1.78343600\end{array}$

$\mathrm{H} \quad \begin{array}{llll}0.00131300 & 1.77641800 & 1.93330900\end{array}$

O $\quad 3.23236400 \quad-0.54540300 \quad-0.97755700$

$\mathrm{H} \quad 4.13965500 \quad-0.30452100 \quad-0.73337000$

$\mathrm{H} \quad 2.98243200 \quad-0.35755900 \quad 1.06095200$ 


\begin{tabular}{lrrr}
\hline \multicolumn{4}{c}{ 5-8-diOH Guanine Neutral (70) } \\
Done: & $\mathrm{E}(\mathrm{R} 3 \mathrm{LYP})=$ & -693.058891811 \\
$0 \mathrm{1}$ & & & \\
$\mathrm{C}$ & 0.32326500 & 0.56882400 & 0.37254000 \\
$\mathrm{C}$ & 0.15797400 & -0.91038300 & 0.07341400 \\
$\mathrm{C}$ & -2.04601800 & -0.62881300 & -0.15957700 \\
$\mathrm{C}$ & -0.79730700 & 1.31889700 & -0.37482400 \\
$\mathrm{C}$ & 2.25942000 & -0.27095100 & -0.15510500 \\
$\mathrm{~N}$ & -1.99080300 & 0.67751600 & -0.45474000 \\
$\mathrm{~N}$ & -0.96667400 & -1.51768900 & -0.07982200 \\
$\mathrm{O}$ & -0.62481900 & 2.48346000 & -0.76531400 \\
$\mathrm{~N}$ & 1.41185500 & -1.39353900 & -0.08022300 \\
$\mathrm{~N}$ & -3.23682400 & -1.20334300 & -0.03131200 \\
$\mathrm{H}$ & -4.08106500 & -0.65117800 & -0.11182000 \\
$\mathrm{H}$ & -3.30704100 & -2.19936500 & 0.13177100 \\
$\mathrm{~N}$ & 1.70406200 & 0.87537200 & 0.03932600 \\
$\mathrm{H}$ & 1.65216000 & -2.33155700 & -0.39034700 \\
$\mathrm{O}$ & 0.07246300 & 0.72581800 & 1.77155800 \\
$\mathrm{H}$ & 0.11923300 & 1.67396400 & 1.98106400 \\
$\mathrm{O}$ & 3.54950400 & -0.41889000 & -0.42455800 \\
$\mathrm{H}$ & 3.80420800 & -1.34864800 & -0.55544900
\end{tabular}

4-NHCH3-gua-mN3H-rad-smd (A24)

4-NHCH3 Guanine Radical (72)

\begin{tabular}{lrrr}
\hline SCF Done: & E(UB3LYP) $=$ & -637.844803826 a.u. \\
$0 ~ 2$ & & & \\
$\mathrm{C}$ & 0.20016300 & 1.12277000 & 0.11839000 \\
$\mathrm{C}$ & 0.73623400 & -0.27619900 & -0.09333700 \\
$\mathrm{C}$ & -1.45726500 & -0.94372100 & -0.44675400 \\
$\mathrm{C}$ & -1.17328500 & 1.29833600 & 0.48529300 \\
$\mathrm{C}$ & 2.04501600 & 1.41218700 & -0.90603800 \\
$\mathrm{~N}$ & -1.91246700 & 0.12715300 & 0.33298900 \\
$\mathrm{~N}$ & -0.21520500 & -1.13852200 & -0.77249900 \\
$\mathrm{O}$ & -1.71495000 & 2.35227800 & 0.88229100 \\
$\mathrm{~N}$ & 1.88613800 & 0.07847900 & -0.94076700 \\
$\mathrm{~N}$ & -2.43802100 & -1.82411800 & -0.81139200 \\
$\mathrm{H}$ & -3.37392200 & -1.45841800 & -0.94208300 \\
$\mathrm{H}$ & -2.15595200 & -2.52873300 & -1.48130700 \\
$\mathrm{~N}$ & 1.05227300 & 2.08537800 & -0.32059700 \\
$\mathrm{H}$ & 2.63628000 & -0.56460300 & -1.16575900 \\
$\mathrm{H}$ & 2.90242600 & 1.89086900 & -1.36148000 \\
$\mathrm{~N}$ & 1.23468800 & -0.82631500 & 1.18403000 \\
$\mathrm{H}$ & 0.43268900 & -0.86188800 & 1.81297800 \\
$\mathrm{C}$ & 1.81247100 & -2.18080400 & 1.09829300 \\
$\mathrm{H}$ & 2.08551100 & -2.49577700 & 2.10829300 \\
$\mathrm{H}$ & 1.13289000 & -2.92981400 & 0.67577800 \\
$\mathrm{H}$ & 2.72975200 & -2.16595600 & 0.50245300 \\
$\mathrm{H}$ & -2.90192400 & 0.18629900 & 0.55536000
\end{tabular}

4-NHCH 3 N3H Guanine Radical Cation (71)

SCF Done: $\mathrm{E}(\mathrm{UB} 3 \mathrm{LYP})=-638.302227108$ a.u.

12

$\mathrm{C}$

C

C

C

C

$\mathrm{N}$

N

$\mathrm{O}$

$\mathrm{N}$

$\mathrm{N}$

$\mathrm{H}$

$\mathrm{H}$

$\mathrm{N}$

$\mathrm{H}$

$\mathrm{H}$

N

$\mathrm{H}$

C

$\mathrm{H}$

H

H

$\mathrm{H}$

$\mathrm{H}$

$\begin{array}{rrr}0.31997300 & 1.13106800 & 0.14151000 \\ 0.76307000 & -0.30524800 & -0.05387500 \\ -1.59225800 & -0.78491400 & -0.43488000 \\ -1.04198300 & 1.43324000 & 0.45861600 \\ 2.19944800 & 1.26059900 & -0.85341800 \\ -1.90870500 & 0.32984500 & 0.28142600 \\ -0.31631400 & -0.99735800 & -0.79220300 \\ -1.50126700 & 2.52313500 & 0.82485400 \\ 1.93185600 & -0.06020700 & -0.89488000 \\ -2.55527100 & -1.62833800 & -0.77789100 \\ -3.52805700 & -1.39807900 & -0.61178900 \\ -2.33650900 & -2.48558300 & -1.27182500 \\ 1.25929900 & 2.01118100 & -0.28212500 \\ 2.64967300 & -0.75898800 & -1.06026300 \\ 3.10568200 & 1.66152500 & -1.28819700 \\ 1.14894100 & -0.93790500 & 1.19320600 \\ 0.37414100 & -0.83407100 & 1.84760500 \\ 1.51034200 & -2.36580300 & 1.09876500 \\ 1.80157700 & -2.70451300 & 2.09463700 \\ 0.69156600 & -3.00385100 & 0.74361100 \\ 2.37157200 & -2.49641600 & 0.43898000 \\ -0.10293500 & -1.88611100 & -1.23883700 \\ -2.88677900 & 0.48682900 & 0.51422100\end{array}$

\section{4-NHCH3 Guanine Radical (73)}

SCF Done: $\mathrm{E}(\mathrm{UB} 3 \mathrm{LYP})=-637.845666570$ a.u.

02

$\begin{array}{llll}\text { C } & 0.30299300 & 1.11298400 & 0.13738600\end{array}$

$\begin{array}{llll}\text { C } & 0.72046700 & -0.32333900 & -0.08359200\end{array}$

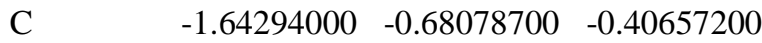

$\begin{array}{llll}\text { C } & -1.07778600 & 1.42504600 & 0.43543900\end{array}$

$\begin{array}{llll}\text { C } & 2.21876700 & 1.22432800 & -0.80073400\end{array}$

$\begin{array}{llll}\mathrm{N} & -2.00754500 & 0.43535800 & 0.22543300\end{array}$

$\begin{array}{llll}\mathrm{N} & -0.36769700 & -0.96653400 & -0.82498600\end{array}$

$\begin{array}{llll}\mathrm{O} & -1.43074100 & 2.56375300 & 0.84626100\end{array}$

$\begin{array}{llll}\mathrm{N} & 1.91394600 & -0.09470800 & -0.89740400\end{array}$

$\mathrm{N} \quad \quad \quad-2.59248000 \quad-1.59329900 \quad-0.68157500$

$\mathrm{H} \quad-3.56108600 \quad-1.33573900-0.55069500$

$\mathrm{H} \quad \begin{array}{llll}-2.37870300 & -2.42640100 & -1.21353100\end{array}$

$\mathrm{N} \quad \begin{array}{llll}1.28268900 & 1.97976200 & -0.23867100\end{array}$

$\mathrm{H} \quad 2.63635400 \quad-0.79795800 \quad-1.00969100$

$\mathrm{H} \quad 3.14972200 \quad 1.61161400 \quad-1.19383700$

$\begin{array}{llll}\mathrm{N} & 1.08380000 & -0.97797300 & 1.17553900\end{array}$

$\begin{array}{llll}\mathrm{H} & 0.28180800 & -0.88704500 & 1.79881000\end{array}$

$\begin{array}{llll}\text { C } & 1.43081300 & -2.40741600 & 1.06456400\end{array}$

$\begin{array}{llll}\mathrm{H} & 1.65653100 & -2.78048500 & 2.06553800\end{array}$

$\begin{array}{llll}\mathrm{H} & 0.63053700 & -3.02947500 & 0.64259200\end{array}$

$\begin{array}{llll}\mathrm{H} & 2.32923400 & -2.53301300 & 0.45465400\end{array}$

$\mathrm{H} \quad-\quad-0.20134200 \quad-1.91465400 \quad-1.15122000$ 


\section{4-NH2CH3 Oxidized Guanine Cation (74)}

SCF Done: $\mathrm{E}(\mathrm{RB} 3 \mathrm{LYP})=-637.663022525$ a.u.

11

C

C

C

C

C

$\mathrm{C}$

$\mathrm{H}$

$\mathrm{H}$

$\mathrm{N}$

$\mathrm{N}$

$\mathrm{O}$

$\mathrm{N}$

$\mathrm{N}$

$\mathrm{N}$

$\mathrm{H}$

$\mathrm{H}$

$\mathrm{N}$

$\mathrm{H}$

$\mathrm{C}$

$\mathrm{H}$

$\mathrm{H}$

$\mathrm{H}$

$\mathrm{H}$ $\begin{array}{lll}0.06884900 & 1.12424000 & 0.05415800\end{array}$

$-0.72638000 \quad-0.14925000 \quad-0.11235100$

$\begin{array}{lll}1.37540000 & -1.05811800 & -0.46737800\end{array}$

$\begin{array}{lll}1.45746200 & 1.00405900 & 0.59839900\end{array}$

$\begin{array}{lll}-1.63334400 & 1.66325200 & -1.11594300\end{array}$

$\begin{array}{llll}-2.31297000 & 2.28852300 & -1.68207600\end{array}$

$\begin{array}{lll}-2.63028000 & -0.13926500 & -1.27638900\end{array}$

$\begin{array}{lll}2.07820400 & -0.13323900 & 0.27144400\end{array}$

$\begin{array}{lll}0.05611100 & -1.11768700 & -0.74484700\end{array}$

$\begin{array}{lll}1.94173800 & 1.93595200 & 1.27498600\end{array}$

$\begin{array}{llll}-0.45643500 & 2.14481100 & -0.55989200\end{array}$

$\begin{array}{lll}-1.82633900 & 0.38926800 & -0.93719600\end{array}$

$\begin{array}{lll}2.12598800 & -2.05236800 & -0.96078300\end{array}$

$3.11660500-2.07646800-0.76368800$

$\begin{array}{lll}1.70093500 & -2.81070200 & -1.47656700\end{array}$

$\begin{array}{lll}-1.35566200 & -0.64207600 & 1.21019200\end{array}$

$\begin{array}{lll}-1.89296400 & 0.12336000 & 1.63977800\end{array}$

$\begin{array}{lll}-2.21759800 & -1.86770500 & 1.09593600\end{array}$

$\begin{array}{lll}-1.64304300 & -2.65432200 & 0.61267700\end{array}$

$\begin{array}{lll}-2.48779100 & -2.16275500 & 2.10919600\end{array}$

$\begin{array}{lll}-3.11298600 & -1.62368500 & 0.52645100\end{array}$

$\begin{array}{lll}-0.57082200 & -0.85212500 & 1.84138200\end{array}$

\section{4-NHCH3 N3H Oxidized Guanine Cation (74')}

SCF Done: $\mathrm{E}(\mathrm{RB} 3 \mathrm{LYP})=-637.676885444$ a.u.

11

C

C

C

C

C

$\mathrm{H}$

$\mathrm{H}$

$\mathrm{N}$

$\mathrm{N}$

$\mathrm{O}$

$\mathrm{N}$

$\mathrm{N}$

$\mathrm{N}$

$\mathrm{H}$

$\mathrm{H}$

$\mathrm{N}$

$\mathrm{H}$

$\mathrm{H}$

C

$\mathrm{H}$

$\mathrm{H}$

$\mathrm{H}$ $\begin{array}{lll}0.36869200 & 0.85240200 & -0.24060400\end{array}$

$\begin{array}{lll}0.56723900 & -0.65441300 & -0.06006200\end{array}$

$\begin{array}{lll}-1.85817700 & -0.59194900 & -0.16170200\end{array}$

$\begin{array}{lll}-0.87810600 & 1.47547500 & 0.29977000\end{array}$

$\begin{array}{llll}2.00382200 & 0.34258700 & -1.51262100\end{array}$

$\begin{array}{lll}2.82391700 & 0.48569700 & -2.20613000\end{array}$

$\begin{array}{lll}2.16273000 & -1.68898600 & -1.21264400\end{array}$

$\begin{array}{llll}-1.96859600 & 0.68179800 & 0.24969300\end{array}$

$\begin{array}{lll}-0.68307600 & -1.22211900 & -0.50254100\end{array}$

$\begin{array}{lll}-0.87259400 & 2.64411800 & 0.71179700\end{array}$

$\begin{array}{lll}1.21260500 & 1.39980700 & -1.05745900\end{array}$

$\begin{array}{lll}1.65574600 & -0.81796400 & -1.05286400\end{array}$

$\begin{array}{lll}-2.97089300 & -1.30984100 & -0.26327100\end{array}$

$\begin{array}{lll}-3.85701400 & -0.86857000 & -0.05372600\end{array}$

$\begin{array}{lll}-2.95605900 & -2.28455000 & -0.53858900\end{array}$

$\begin{array}{lll}0.98813700 & -1.19479800 & 1.20763700\end{array}$

$\begin{array}{llll}0.19394700 & -1.13250500 & 1.84430400\end{array}$

$\begin{array}{lll}-0.70665000 & -2.23481800 & -0.60735500\end{array}$

$\begin{array}{lll}2.17059500 & -0.57166900 & 1.83772800\end{array}$

$3.05542000 \quad-0.73213600 \quad 1.21935800$

$\begin{array}{lll}2.05073100 & 0.50228900 & 2.02370200\end{array}$

$\begin{array}{lll}2.33187700 & -1.07214600 & 2.79329600\end{array}$
4-NH2 $\mathrm{NH}_{2}$ Oxidized Guanine Neutral (75)

SCF Done: $E($ RB3LYP $)=-637.181042987$ a.u.

01

$\begin{array}{llll}\text { C } & 0.23056700 & 0.82781500 & -0.30621100\end{array}$

$\begin{array}{llll}\text { C } & 0.48320800 & -0.63858600 & -0.15422800\end{array}$

$\begin{array}{llll}\text { C } & -1.87931500 & -0.73502100 & -0.16546000\end{array}$

$\begin{array}{llll}\text { C } & -1.00066700 & 1.37935500 & 0.31447900\end{array}$

$\begin{array}{llll}\mathrm{C} & 1.89166800 & 0.40848700 & -1.57718900\end{array}$

$\begin{array}{llll}\mathrm{N} & -2.01883700 & 0.53451400 & 0.40842700\end{array}$

$\mathrm{N} \quad \quad \quad-0.66766900 \quad-1.29575000 \quad-0.57264400$

$\begin{array}{llll}\mathrm{O} & -0.99489600 & 2.58122500 & 0.71743800\end{array}$

$\mathrm{N} \quad \begin{array}{llll}0 & 1.61659000 & -0.77188500 & -1.07255000\end{array}$

$\mathrm{N} \quad \quad-2.97687100 \quad-1.42675500 \quad-0.31784500$

$\mathrm{H} \quad-2.73463900 \quad-2.30872800 \quad-0.77462000$

$\mathrm{N} \quad \begin{array}{llll}\mathrm{N} & 1.05067400 & 1.40595300 & -1.16420600\end{array}$

$\mathrm{H} \quad 2.12456900 \quad-1.63308000 \quad-1.26354700$

$\mathrm{H} \quad \begin{array}{llll}2.68551200 & 0.56208600 & -2.29718800\end{array}$

$\begin{array}{llll}\mathrm{N} & 0.97613100 & -1.09424700 & 1.26207600\end{array}$

$\begin{array}{llll}\mathrm{H} & 0.17322600 & -0.97987100 & 1.89192500\end{array}$

$\begin{array}{llll}\text { C } & 2.16850400 & -0.39710300 & 1.84486700\end{array}$

$\begin{array}{llll}\mathrm{H} & 2.35049700 & -0.81495200 & 2.83469400\end{array}$

$\begin{array}{llll}\mathrm{H} & 3.03193800 & -0.57037800 & 1.20524900\end{array}$

$\begin{array}{llll}\mathrm{H} & 1.95068600 & 0.66724200 & 1.92714000\end{array}$

$\begin{array}{llll}\mathrm{H} & 1.15346300 & -2.10460200 & 1.19649000\end{array}$

\section{4-NHCH${ }_{3}$ Oxidized Guanine Neutral (76)}

SCF Done: $\mathrm{E}(\mathrm{R} 3 \mathrm{LYP})=-637.221348808$ a.u.

01

$\mathrm{C}$

$\begin{array}{lll}0.33893300 & 0.78096600 & -0.32877300\end{array}$

$\begin{array}{lll}0.52483900 & -0.67474000 & 0.02481700\end{array}$

$\begin{array}{llll}\text { C } & -1.79810500 & -0.66417100 & -0.08833700\end{array}$

$\begin{array}{llll}\text { C } & -0.88180600 & 1.44963500 & 0.21519200\end{array}$

$\begin{array}{lllll}\mathrm{C} & & 1.92926200 & 0.13568700 & -1.60096000\end{array}$

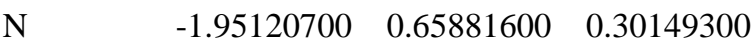

$\mathrm{N}$

$\mathrm{O}$

$\begin{array}{lll}-0.68647000 & -1.36238100 & -0.30975900\end{array}$

$\begin{array}{lll}-0.83822100 & 2.66358500 & 0.53369600\end{array}$

$\begin{array}{lll}1.60050900 & -0.96317100 & -1.00027700\end{array}$

$\begin{array}{llll}-2.97374700 & -1.31067700 & -0.24684000\end{array}$

$\begin{array}{lll}-3.83347200 & -0.83785300 & -0.01070000\end{array}$

$\begin{array}{llll}-2.98581600 & -2.30420400 & -0.42835700\end{array}$

$\begin{array}{lll}1.11479900 & 1.21890600 & -1.27769300\end{array}$

$2.12150800-1.83666200-1.06137800$

$\begin{array}{llll}2.74182100 & 0.21163900 & -2.31355500\end{array}$

$0.95812000 \quad-0.94700200 \quad 1.36778800$

$\begin{array}{lll}2.17631300 & -0.26217500 & 1.82997700\end{array}$

$\begin{array}{lll}2.39079900 & -0.60276000 & 2.84431200\end{array}$

$\begin{array}{lll}3.06135100 & -0.45029000 & 1.20879700\end{array}$

$\begin{array}{lll}1.99958100 & 0.81654400 & 1.86649800\end{array}$

$\begin{array}{lll}1.03936300 & -1.95774600 & 1.47033200\end{array}$ 


\begin{tabular}{lrrr}
\hline \multicolumn{4}{c}{ 4-NCH 3 Oxidized Guanine Neutral (77) } \\
\hline SCF Done: & E(R3LYP) $=$ & -637.185138615 & a.u. \\
O 1 & & & \\
$\mathrm{C}$ & 0.18614800 & 1.11580100 & 0.08287100 \\
$\mathrm{C}$ & 0.76540800 & -0.28050500 & 0.07836600 \\
$\mathrm{C}$ & -1.53818700 & -0.84689000 & -0.44726100 \\
$\mathrm{C}$ & -1.22829800 & 1.25505300 & 0.53670900 \\
$\mathrm{C}$ & 2.00296400 & 1.34637700 & -1.02306300 \\
$\mathrm{~N}$ & -2.03895500 & 0.22755100 & 0.20364000 \\
$\mathrm{~N}$ & -0.22505300 & -1.03932400 & -0.75940200 \\
$\mathrm{O}$ & -1.60796500 & 2.26845900 & 1.15353100 \\
$\mathrm{~N}$ & 1.96200500 & 0.06112500 & -0.84220000 \\
$\mathrm{~N}$ & -2.41050100 & -1.78211100 & -0.83075000 \\
$\mathrm{H}$ & -3.39768800 & -1.63292800 & -0.67116600 \\
$\mathrm{H}$ & -2.10847900 & -2.61866400 & -1.31342200 \\
$\mathrm{~N}$ & 0.87505100 & 2.01344800 & -0.56018900 \\
$\mathrm{H}$ & 2.75999500 & -0.54842600 & -1.00750400 \\
$\mathrm{H}$ & 2.84742600 & 1.87106500 & -1.45233000 \\
$\mathrm{~N}$ & 1.13138000 & -0.76323100 & 1.30220400 \\
$\mathrm{C}$ & 1.77689700 & -2.07586500 & 1.25439300 \\
$\mathrm{H}$ & 2.02880800 & -2.38170000 & 2.27359100 \\
$\mathrm{H}$ & 1.13024900 & -2.86536300 & 0.83745800 \\
$\mathrm{H}$ & 2.71970700 & -2.10371700 & 0.68352300 \\
$\mathrm{H}$ & 0.03662300 & -1.97397300 & -1.06360100 \\
& & & \\
\hline & & & \\
\hline & $4-N C H 3$ & Sp Neutral (78) \\
\hline
\end{tabular}

SCF Done: $\mathrm{E}(\mathrm{R} 3 \mathrm{LYP})=-637.280999745$ a.u.

01

$\begin{array}{lrrr}\mathrm{C} & 0.06718700 & -0.48711700 & -0.32704600 \\ \mathrm{C} & -1.19122100 & 0.36099000 & -0.09408000 \\ \mathrm{C} & 2.16049900 & 0.39484900 & -0.24669200 \\ \mathrm{C} & 0.82584100 & -0.73880200 & 1.01865500 \\ \mathrm{~N} & 2.05741300 & -0.17384000 & 0.96999200 \\ \mathrm{~N} & 1.11094500 & 0.16263800 & -1.08378100 \\ \mathrm{O} & 0.32785300 & -1.36412300 & 1.96149400 \\ \mathrm{~N} & -2.23291100 & -0.51970900 & -0.36323300 \\ \mathrm{~N} & 3.21487400 & 1.10786700 & -0.61894300 \\ \mathrm{H} & 3.97259300 & 1.25339400 & 0.03462000 \\ \mathrm{H} & 3.26881900 & 1.52387700 & -1.53979800 \\ \mathrm{~N} & -1.21661100 & 1.58044000 & 0.27800100 \\ \mathrm{H} & 0.89718600 & 0.79427600 & -1.84871300 \\ \mathrm{C} & -2.53319500 & 2.19372600 & 0.45888700 \\ \mathrm{H} & -3.10902600 & 2.20062500 & -0.47540600 \\ \mathrm{H} & -3.12583400 & 1.66637700 & 1.21713600 \\ \mathrm{H} & -2.40925500 & 3.22681000 & 0.78644300 \\ \mathrm{C} & -1.69751000 & -1.69161100 & -0.85450900 \\ \mathrm{~N} & -0.40979200 & -1.75357900 & -0.90180500 \\ \mathrm{H} & -3.21727500 & -0.28273400 & -0.38092000 \\ \mathrm{H} & -2.35706200 & -2.48857700 & -1.17822400\end{array}$

4-NCH3-8-NHCH3 Sp Neutral (79)
SCF Done: $\mathrm{E}(\mathrm{R} 3 \mathrm{LYP})=-733.169460842$ a.u.

01

$\begin{array}{llll}\text { C } & 0.45742100 & -0.36350300 & -0.16722600\end{array}$

$\begin{array}{lllll}\mathrm{C} & -0.24110100 & 0.99106000 & -0.01029000\end{array}$

$\begin{array}{llll}\text { C } & 2.71615300 & -0.45719600 & -0.43768800\end{array}$

$\begin{array}{llll}\mathrm{C} & 1.25561000 & -0.72965500 & 1.12387400\end{array}$

$\begin{array}{llll}\mathrm{N} & 2.58836300 & -0.75006000 & 0.87247700\end{array}$

$\mathrm{N} \quad \begin{array}{llll}1.54770000 & -0.36481400 & -1.12838400\end{array}$

$\begin{array}{llll}\mathrm{O} & 0.70027300 & -0.97339500 & 2.20530600\end{array}$

$\begin{array}{llll}\mathrm{N} & -1.58095300 & 0.73395900 & 0.01861200\end{array}$

$\mathrm{N} \quad 3.89589800 \quad-0.30246800 \quad-1.02596100$

$\mathrm{H} \quad 4.74196200 \quad-0.38879300 \quad-0.47912300$

$\mathrm{H} \quad 3.96601800 \quad-0.09517500 \quad-2.01370100$

$\begin{array}{llll}\mathrm{N} & 0.39698400 & 2.10166600 & 0.06584300\end{array}$

$\begin{array}{llll}\mathrm{H} & 1.49230800 & 0.18348300 & -1.98076300\end{array}$

$\begin{array}{lllll}\text { C } & -0.41673700 & 3.30474400 & 0.23019900\end{array}$

$\mathrm{H} \quad-1.11427600 \quad 3.44803100 \quad-0.60682900$

$\begin{array}{llll}\mathrm{H} & -1.01015600 & 3.28182200 & 1.15547000\end{array}$

$\begin{array}{llll}\mathrm{H} & 0.23346600 & 4.18062800 & 0.27550500\end{array}$

$\begin{array}{llll}\text { C } & -1.88677100 & -0.70276300 & 0.03549800\end{array}$

$\begin{array}{lllll}\text { C } & -4.30728000 & -0.68018000 & -0.18399900\end{array}$

$\mathrm{H} \quad-4.42703700 \quad-1.11347000 \quad 0.81325100$

$\mathrm{H} \quad-4.41659300 \quad 0.41127500 \quad-0.10096400$

$\mathrm{H} \quad-5.11923100 \quad-1.05276100 \quad-0.81252900$

$\mathrm{N} \quad-3.02125000 \quad-1.10861700 \quad-0.75487900$

$\mathrm{H} \quad-2.91948700 \quad-0.71645500 \quad-1.69061400$

$\mathrm{N} \quad-0.63767900 \quad-1.26801000 \quad-0.49165500$

$\begin{array}{llll}\mathrm{H} & -2.24377900 & 1.39929100 & 0.39600600\end{array}$

$\mathrm{H} \quad-2.08566700 \quad-1.02356500 \quad 1.06508900$

$\mathrm{H} \quad-0.48692100 \quad-2.19377800 \quad-0.09783600$

\section{4-NCH $3-8-\mathrm{NHCH}_{3}$ Sp Radical Neutral (80)}

$\begin{array}{lrrr}\text { SCF Done: } & \mathrm{E}(\mathrm{U} 3 \mathrm{LYP})= & -732.512680935 \\ 0 \mathrm{a} . \mathrm{u} . \\ \mathrm{C} & 0.33333700 & -0.19142800 & -0.39310300 \\ \mathrm{C} & -0.10113200 & 1.25593400 & -0.13939200 \\ \mathrm{C} & 2.51242200 & -0.83672800 & -0.21790000 \\ \mathrm{C} & 0.70909900 & -0.89786300 & 0.97727200 \\ \mathrm{~N} & 2.02115100 & -1.22358600 & 0.97602400 \\ \mathrm{~N} & 1.58831500 & -0.35366300 & -1.08943600 \\ \mathrm{O} & -0.11462400 & -1.08881900 & 1.87622100 \\ \mathrm{~N} & -1.42732100 & 1.29490000 & -0.46573700 \\ \mathrm{~N} & 3.79556700 & -0.94402400 & -0.53364200 \\ \mathrm{H} & 4.44685700 & -1.30655400 & 0.14966200 \\ \mathrm{H} & 4.14068400 & -0.64680100 & -1.43734800 \\ \mathrm{~N} & 0.67939000 & 2.17439300 & 0.29643000 \\ \mathrm{H} & 1.84887400 & 0.25302800 & -1.85965000 \\ \mathrm{C} & 0.07283400 & 3.48902700 & 0.50374700 \\ \mathrm{H} & -0.32675500 & 3.90944500 & -0.42933500 \\ \mathrm{H} & -0.75004600 & 3.45295300 & 1.23136700 \\ \mathrm{H} & 0.82510700 & 4.18107700 & 0.88721800 \\ \mathrm{C} & -1.95355400 & -0.02268100 & -0.79670600 \\ \mathrm{C} & -3.66720800 & -1.66581600 & -0.10817400\end{array}$




$\begin{array}{llll}\mathrm{H} & -4.35269900 & -1.31023300 & -0.88278600 \\ \mathrm{H} & -3.15774700 & -2.56284000 & -0.48203600 \\ \mathrm{H} & -4.25928700 & -1.95251300 & 0.76445800 \\ \mathrm{~N} & -2.76321400 & -0.57759200 & 0.28095300 \\ \mathrm{H} & -2.14966200 & -0.86852100 & 1.04461000 \\ \mathrm{~N} & -0.77456400 & -0.83834500 & -1.05064000 \\ \mathrm{H} & -2.04086100 & 2.03858900 & -0.15616600 \\ \mathrm{H} & -2.57752600 & 0.01566500 & -1.69187600\end{array}$

\begin{tabular}{|c|c|c|c|}
\hline \multicolumn{4}{|c|}{ 4-NCH3-8-NHCH3 Sp Radical (81) } \\
\hline \multicolumn{4}{|c|}{$\begin{array}{l}\text { SCF Done: } \mathrm{E}(\mathrm{U} 3 \mathrm{LYP})=-732.512025366 \text { a.u. } \\
02\end{array}$} \\
\hline $\mathrm{C}$ & 0.44997100 & -0.34317600 & -0.16908100 \\
\hline $\mathrm{C}$ & -0.26793800 & 1.00626900 & -0.03849500 \\
\hline $\mathrm{C}$ & 2.71267400 & -0.46875000 & -0.40104500 \\
\hline $\mathrm{C}$ & 1.22373000 & -0.71150900 & 1.14091200 \\
\hline $\mathrm{N}$ & 2.55892300 & -0.75382500 & 0.90800700 \\
\hline $\mathrm{N}$ & 1.55663200 & -0.35401400 & -1.10984100 \\
\hline $\mathrm{O}$ & 0.64704600 & -0.93801700 & 2.21336400 \\
\hline $\mathrm{N}$ & -1.60543500 & 0.71743900 & -0.03352200 \\
\hline $\mathrm{N}$ & 3.90330800 & -0.34216800 & -0.97288300 \\
\hline $\mathrm{H}$ & 4.73970300 & -0.44249000 & -0.41361100 \\
\hline $\mathrm{H}$ & 3.99122200 & -0.14094800 & -1.96057900 \\
\hline $\mathrm{N}$ & 0.34882600 & 2.12862800 & 0.06593500 \\
\hline $\mathrm{H}$ & 1.52131400 & 0.18748400 & -1.96765000 \\
\hline $\mathrm{C}$ & -0.48654000 & 3.31898700 & 0.20196600 \\
\hline $\mathrm{H}$ & -1.17375800 & 3.44149100 & -0.64742800 \\
\hline $\mathrm{H}$ & -1.09361600 & 3.29470400 & 1.11799600 \\
\hline $\mathrm{H}$ & 0.14865200 & 4.20569000 & 0.24745300 \\
\hline $\mathrm{C}$ & -1.86760200 & -0.67136900 & -0.11180500 \\
\hline $\mathrm{C}$ & -4.29004700 & -0.76702700 & -0.04113000 \\
\hline $\mathrm{H}$ & -4.28070500 & -1.06480300 & 1.01064200 \\
\hline $\mathrm{H}$ & -4.46702800 & 0.31714100 & -0.09404500 \\
\hline $\mathrm{H}$ & -5.12531400 & -1.26905800 & -0.53513900 \\
\hline $\mathrm{N}$ & -3.03820000 & -1.19612200 & -0.67811300 \\
\hline $\mathrm{H}$ & -3.07178400 & -1.02321700 & -1.68872400 \\
\hline $\mathrm{N}$ & -0.63459000 & -1.25455800 & -0.49840100 \\
\hline $\mathrm{H}$ & -2.33093100 & 1.40420300 & 0.12708900 \\
\hline $\mathrm{H}$ & -0.50585600 & -2.20427100 & -0.15912600 \\
\hline
\end{tabular}

$\begin{array}{lrrr}\mathrm{N} & 3.63498100 & -1.04736200 & -1.04886100 \\ \mathrm{H} & 4.47051200 & -1.24034000 & -0.51323100 \\ \mathrm{H} & 3.69855900 & -0.97727100 & -2.05593300 \\ \mathrm{~N} & 0.58676200 & 2.23386300 & -0.13772500 \\ \mathrm{H} & 1.32383000 & -0.18374000 & -2.00705700 \\ \mathrm{C} & -0.08970000 & 3.53210200 & -0.10731100 \\ \mathrm{H} & -0.68298000 & 3.65810900 & 0.80755200 \\ \mathrm{H} & 0.65324800 & 4.33035500 & -0.13987400 \\ \mathrm{H} & -0.76307100 & 3.66190800 & -0.96500100 \\ \mathrm{C} & -1.90668400 & -0.23341100 & -0.13891700 \\ \mathrm{C} & -3.68340300 & -1.90753500 & -0.40166900 \\ \mathrm{H} & -4.76791600 & -1.91786300 & -0.29113000 \\ \mathrm{H} & -3.42728600 & -2.23504300 & -1.41553400 \\ \mathrm{H} & -3.25583700 & -2.61237700 & 0.31476500 \\ \mathrm{~N} & -3.21566300 & -0.55335200 & -0.12610200 \\ \mathrm{~N} & -0.90486400 & -1.06834900 & -0.21451900 \\ \mathrm{H} & -2.18408700 & 1.88828500 & -0.10051600 \\ \mathrm{H} & -3.86931400 & 0.20353800 & -0.28148100\end{array}$

$\overline{\text { SCF Done: } \mathrm{E}(\mathrm{R} 3 \mathrm{LYP})=-7 \mathrm{diNCH}_{3} \text { Sp (82') }}$

01

$\begin{array}{llll}\text { C } & 0.30015600 & -0.26531700 & -0.09763300\end{array}$

$\begin{array}{lllll}\text { C } & -0.15880000 & 1.20318500 & -0.09214900\end{array}$

$\begin{array}{lllll}\mathrm{C} & 2.48378300 & -0.84783900 & -0.41316900\end{array}$

$\begin{array}{llll}\mathrm{C} & 1.08418400 & -0.60538000 & 1.21290800\end{array}$

$\begin{array}{llll}\mathrm{N} & 2.36637800 & -0.94217400 & 0.92718900\end{array}$

$\mathrm{N} \quad \begin{array}{llll}1.33299200 & -0.56389400 & -1.07348000\end{array}$

$\begin{array}{llll}\mathrm{O} & 0.56316100 & -0.56121200 & 2.33582000\end{array}$

$\mathrm{N} \quad-1.53901700 \quad 1.12191300 \quad-0.04917700$

$\mathrm{N} \quad 3.63482900 \quad-1.04734700 \quad-1.04468900$

$\mathrm{H} \quad 4.46635300 \quad-1.25046600 \quad-0.50659100$

$\mathrm{H} \quad 3.70489100 \quad-0.96352600 \quad-2.05031100$

$\mathrm{N} \quad \begin{array}{llll}0.60108300 & 2.22911700 & -0.09852900\end{array}$

$\mathrm{H} \quad \begin{array}{llll}1.33119400 & -0.16603300 & -2.00624400\end{array}$

$\begin{array}{lllll}\text { C } & -0.05945100 & 3.53479000 & -0.05788000\end{array}$

$\mathrm{H} \quad-0.66222800 \quad 3.65385500 \quad 0.85172300$

$\mathrm{H} \quad 0.69419000 \quad 4.32355500 \quad-0.06864100$

$\mathrm{H} \quad-0.72063500 \quad 3.68693900 \quad-0.92118100$

C $\quad-1.90940100 \quad-0.21734600 \quad-0.19353800$

C $\quad-3.68757100 \quad-1.90485400 \quad-0.30297300$

$\mathrm{H} \quad-4.77070800-1.89107600 \quad-0.42525500$

$\mathrm{H} \quad-3.25244900-2.45933800-1.13750900$

$\mathrm{H} \quad-3.44005400 \quad-2.42130000 \quad 0.63102400$

$\mathrm{N} \quad-3.21617500 \quad-0.52428000 \quad-0.30308000$

$\mathrm{H} \quad-3.88039800 \quad 0.19663200 \quad-0.05254400$

$\mathrm{N} \quad \begin{array}{llll}-0.91197000 & -1.06004800 & -0.23741400\end{array}$

$\mathrm{H} \quad-2.16967300 \quad 1.90402100 \quad-0.18016800$

4-NCH3, 8-OH Sp Neutral (83) 


$\begin{array}{lrrr}01 & & & \\ \mathrm{C} & 0.14102600 & -0.38517400 & 0.26483000 \\ \mathrm{C} & -0.87956300 & 0.73403400 & 0.01390900 \\ \mathrm{C} & 2.24182500 & -0.20601000 & -0.60715600 \\ \mathrm{C} & 1.23922500 & 0.01784400 & 1.29245800 \\ \mathrm{~N} & 2.44907200 & 0.11451000 & 0.68519000 \\ \mathrm{~N} & 0.98617200 & -0.64271000 & -0.90281400 \\ \mathrm{O} & 0.99558600 & 0.22038900 & 2.48944900 \\ \mathrm{~N} & -2.12171500 & 0.16923200 & 0.18736800 \\ \mathrm{~N} & 3.19654300 & -0.13111000 & -1.52646900 \\ \mathrm{H} & 4.11735200 & 0.19310300 & -1.26216500 \\ \mathrm{H} & 3.01826400 & -0.37817000 & -2.49136100 \\ \mathrm{~N} & -0.54946600 & 1.93257900 & -0.28967700 \\ \mathrm{H} & 0.61327600 & -0.55281300 & -1.84294700 \\ \mathrm{C} & -1.64315000 & 2.86758300 & -0.55080200 \\ \mathrm{H} & -2.29436900 & 2.52609600 & -1.36728200 \\ \mathrm{H} & -2.27255900 & 3.01734400 & 0.33643100 \\ \mathrm{H} & -1.23084300 & 3.83697200 & -0.83608200 \\ \mathrm{C} & -2.04579400 & -1.27463500 & 0.23295300 \\ \mathrm{~N} & -0.69532300 & -1.48657700 & 0.71016000 \\ \mathrm{H} & -2.95934100 & 0.61183700 & -0.17142300 \\ \mathrm{H} & -0.33370700 & -2.39575800 & 0.43726300 \\ \mathrm{O} & -2.22977200 & -1.88442000 & -1.04880300 \\ \mathrm{H} & -3.17361300 & -2.07388900 & -1.16901300 \\ \mathrm{H} & -2.78935800 & -1.68580600 & 0.91794900\end{array}$

\begin{tabular}{lrrr}
\hline \multicolumn{4}{c}{ 4-NCH3-8-OH-Sp Radical Neutral (84) } \\
\hline SCF Done: & E(U3LYP) $=$ & -713.069291921 & a.u. \\
O 2 & & & \\
$\mathrm{C}$ & 0.17144700 & -0.26109600 & -0.38012200 \\
$\mathrm{C}$ & -0.78498000 & 0.89495900 & -0.07968000 \\
$\mathrm{C}$ & 2.44025400 & -0.08791600 & -0.21548800 \\
$\mathrm{C}$ & 0.79302400 & -0.83369800 & 0.98313400 \\
$\mathrm{~N}$ & 2.13526800 & -0.67405900 & 0.95766000 \\
$\mathrm{~N}$ & 1.39190100 & 0.08220400 & -1.06325500 \\
$\mathrm{O}$ & 0.10426400 & -1.31556100 & 1.87970400 \\
$\mathrm{~N}$ & -2.03679100 & 0.35861800 & -0.22709300 \\
$\mathrm{~N}$ & 3.67275100 & 0.27549300 & -0.53950400 \\
$\mathrm{H}$ & 4.42334800 & 0.14596200 & 0.12559300 \\
$\mathrm{H}$ & 3.86911700 & 0.72399100 & -1.42494200 \\
$\mathrm{~N}$ & -0.41698100 & 2.07440200 & 0.25233100 \\
$\mathrm{H}$ & 1.41057700 & 0.77593700 & -1.80313000 \\
$\mathrm{C}$ & -1.48451900 & 3.04530300 & 0.49494700 \\
$\mathrm{H}$ & -2.14731100 & 3.15426400 & -0.37435400 \\
$\mathrm{H}$ & -2.10518100 & 2.76417400 & 1.35587000 \\
$\mathrm{H}$ & -1.04531200 & 4.02233000 & 0.70382000 \\
$\mathrm{C}$ & -2.01488600 & -0.95351700 & -0.81644400 \\
$\mathrm{~N}$ & -0.60536700 & -1.25398000 & -1.06734600 \\
$\mathrm{H}$ & -2.88280700 & 0.91411500 & -0.20681600 \\
$\mathrm{O}$ & -2.57344600 & -1.89961400 & 0.08385200 \\
$\mathrm{H}$ & -2.91132600 & -2.64983500 & -0.43131500 \\
$\mathrm{H}$ & -2.56514700 & -0.99248700 & -1.76081800
\end{tabular}

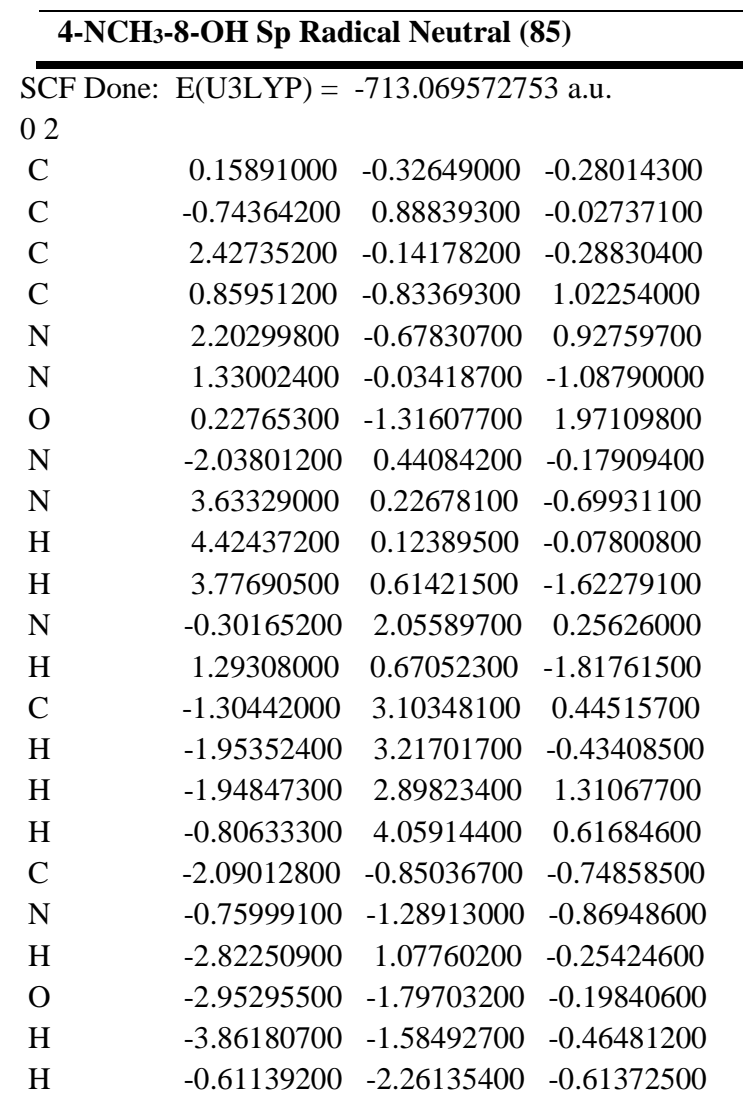


O $\quad-3.04307200 \quad-1.78075500 \quad-0.53339200$

$\mathrm{H} \quad-2.83022600 \quad-2.71611800 \quad-0.69984400$

\begin{tabular}{lrrr}
\hline \multicolumn{4}{c}{ 4-NCH3-8-oxo-Sp Neutral $(\mathbf{8 7})$} \\
\hline SCF Done: & E(R3LYP) $=$ & -712.550508666 & a.u. \\
$0 ~ 1$ & & & \\
C & 0.15428800 & -0.35956700 & -0.21346700 \\
$\mathrm{C}$ & -0.82719500 & 0.81123300 & -0.05022900 \\
$\mathrm{C}$ & 2.40396000 & -0.06967400 & -0.34444700 \\
$\mathrm{C}$ & 0.92928500 & -0.66647000 & 1.11589600 \\
$\mathrm{~N}$ & 2.25443300 & -0.45079200 & 0.93952400 \\
$\mathrm{~N}$ & 1.26714700 & -0.10643900 & -1.09820700 \\
$\mathrm{O}$ & 0.35715400 & -1.05706400 & 2.13845900 \\
$\mathrm{~N}$ & -2.09315500 & 0.25125700 & -0.14124000 \\
$\mathrm{~N}$ & 3.57135100 & 0.29033600 & -0.85543300 \\
$\mathrm{H}$ & 4.39052000 & 0.30422400 & -0.26227300 \\
$\mathrm{H}$ & 3.65934600 & 0.55704000 & -1.82783300 \\
$\mathrm{~N}$ & -0.47646800 & 2.02047700 & 0.14006300 \\
$\mathrm{H}$ & 1.16494100 & 0.47424600 & -1.92462900 \\
$\mathrm{C}$ & -1.54343300 & 3.01156600 & 0.28730600 \\
$\mathrm{H}$ & -2.18039900 & 3.05614200 & -0.60504800 \\
$\mathrm{H}$ & -2.18335400 & 2.79184300 & 1.15078900 \\
$\mathrm{H}$ & -1.09993300 & 3.99683700 & 0.43616900 \\
$\mathrm{C}$ & -2.04695800 & -1.10141500 & -0.43466700 \\
$\mathrm{~N}$ & -0.72964500 & -1.43643600 & -0.62112700 \\
$\mathrm{H}$ & -2.96775200 & 0.75815300 & -0.07298200 \\
$\mathrm{O}$ & -3.01998900 & -1.85133400 & -0.52765200 \\
$\mathrm{H}$ & -0.45601600 & -2.40417000 & -0.48804700 \\
& & &
\end{tabular}

4-OH N3H Guanine Radical Cation (88)

SCF Done: E(UB3LYP) $=-618.862671574$ a.u.

12

C

C

C

$\mathrm{C}$

$\mathrm{N}$

$\mathrm{N}$

$\mathrm{O}$

$\mathrm{N}$

$\mathrm{N}$

$\mathrm{H}$

$\mathrm{H}$

$\mathrm{N}$

$\mathrm{H}$

$\mathrm{H}$

$\mathrm{H}$

$\mathrm{H}$

$\mathrm{O}$

$\mathrm{H}$

$\begin{array}{rrc}0.75509700 & 0.71615400 & 0.06489400 \\ 0.62156700 & -0.77973900 & 0.24163400 \\ -1.71865700 & -0.49345200 & -0.24012700 \\ -0.40667700 & 1.54913000 & 0.13893000 \\ 2.59967400 & -0.09777300 & -0.62200200 \\ -1.61339700 & 0.81171100 & 0.12598300 \\ -0.59919700 & -1.22478000 & -0.40801700 \\ -0.44169400 & 2.78360000 & 0.18807400 \\ 1.83871900 & -1.19762000 & -0.43617800 \\ -2.91435300 & -1.01950700 & -0.44743000 \\ -3.74382200 & -0.43614900 & -0.44698200 \\ -3.01092800 & -2.00299900 & -0.67393900 \\ 1.98434100 & 1.06082200 & -0.37808900 \\ 2.22556500 & -2.13161500 & -0.33524200 \\ 3.62056500 & -0.17384800 & -0.97223200 \\ -0.71169400 & -2.21985000 & -0.59300300 \\ -2.47092400 & 1.35287400 & 0.21663600 \\ 0.63041200 & -1.11874300 & 1.61199800 \\ 0.60267100 & -2.08757700 & 1.71033300\end{array}$

\section{4-OH Guanine Radical (89)}

SCF Done: $\mathrm{E}(\mathrm{UB} 3 \mathrm{LYP})=-618.411872269$ a.u. 02

$\begin{array}{llll}\text { C } & 0.72264600 & 0.70861500 & 0.04714600\end{array}$

$\begin{array}{llll}\text { C } & 0.59466100 & -0.78334000 & 0.23065100\end{array}$

$\begin{array}{llll}\text { C } & -1.64742400 & -0.58899800 & -0.23915500\end{array}$

$\begin{array}{llll}\text { C } & -0.45741800 & 1.51592500 & 0.14990100\end{array}$

$\begin{array}{llll}\mathrm{C} & 2.57201800 & -0.07808400 & -0.65906000\end{array}$

$\begin{array}{llll}\mathrm{N} & -1.62059300 & 0.74824700 & 0.16168700\end{array}$

$\mathrm{N} \quad \begin{array}{llll}\mathrm{N} & -0.59005500 & -1.35018500 & -0.33895200\end{array}$

$\begin{array}{llll}\mathrm{O} & -0.51052000 & 2.76037700 & 0.20897700\end{array}$

$\mathrm{N} \quad \begin{array}{llll}\mathrm{N} & 1.83764800 & -1.18198800 & -0.44241000\end{array}$

$\mathrm{N} \quad \begin{array}{llll}\mathrm{N} & -2.88902000 & -1.07765300 & -0.49310100\end{array}$

$\mathrm{H} \quad-3.62417700 \quad-0.43512400 \quad-0.76208700$

$\begin{array}{lllll}\mathrm{H} & -2.93643000 & -2.00561400 & -0.89319800\end{array}$

$\begin{array}{llll}\mathrm{N} & 1.93732300 & 1.07688800 & -0.42506400\end{array}$

$\mathrm{H} \quad \begin{array}{llll}2.22652400 & -2.11633900 & -0.37747200\end{array}$

$\mathrm{H} \quad 3.58773800 \quad-0.13540700 \quad-1.02808500$

$\begin{array}{llll}\mathrm{O} & 0.68285000 & -1.09651000 & 1.62832700\end{array}$

$\mathrm{H} \quad \begin{array}{llll}\mathrm{H} & 0.43074900 & -2.03009500 & 1.73542200\end{array}$

$\begin{array}{llll}\mathrm{H} & -2.49705000 & 1.25975900 & 0.21496700\end{array}$

\begin{tabular}{lrrr}
\hline \multicolumn{4}{c}{ 4-OH Guanine Radical (90) } \\
\hline SCF Done: & E(UB3LYP) $=$ & -618.408269367 a.u. \\
0 2 & & & \\
$\mathrm{C}$ & 0.72207600 & 0.70174900 & 0.06550800 \\
$\mathrm{C}$ & 0.57718100 & -0.79107100 & 0.21470500 \\
$\mathrm{C}$ & -1.73763600 & -0.38238800 & -0.23485000 \\
$\mathrm{C}$ & -0.45675400 & 1.53942300 & 0.13795300 \\
$\mathrm{C}$ & 2.57705400 & -0.11753700 & -0.60670500 \\
$\mathrm{~N}$ & -1.67747100 & 0.91378000 & 0.06529200 \\
$\mathrm{~N}$ & -0.64586200 & -1.19867800 & -0.42689500 \\
$\mathrm{O}$ & -0.37782600 & 2.79150800 & 0.24133700 \\
$\mathrm{~N}$ & 1.79647400 & -1.21915500 & -0.45930900 \\
$\mathrm{~N}$ & -2.94346600 & -0.94584400 & -0.40464700 \\
$\mathrm{H}$ & -3.76537200 & -0.35807900 & -0.39224400 \\
$\mathrm{H}$ & -3.04446000 & -1.92648400 & -0.62977700 \\
$\mathrm{~N}$ & 1.96887400 & 1.03842900 & -0.35273400 \\
$\mathrm{H}$ & 2.18718700 & -2.14633600 & -0.32442700 \\
$\mathrm{H}$ & 3.60310500 & -0.19881700 & -0.94036400 \\
$\mathrm{H}$ & -0.81994300 & -2.19967200 & -0.48076400 \\
$\mathrm{O}$ & 0.59526000 & -1.15069300 & 1.59416000 \\
$\mathrm{H}$ & 0.51864500 & -2.11790200 & 1.67197900
\end{tabular}

4-OH N3H oxidized Guanine Cation (91)

SCF Done: $\mathrm{E}(\mathrm{RB} 3 \mathrm{LYP})=-618.231438860$ a.u.

11

$\begin{array}{lllll}\text { C } & -0.66661100 & 0.74283800 & -0.03746200\end{array}$

$\begin{array}{llll}\text { C } & -0.64924300 & -0.72715000 & 0.36098200\end{array}$

$\begin{array}{llll}\text { C } & 1.67058600 & -0.51282100 & -0.26585400\end{array}$

$\begin{array}{lllll}\mathrm{C} & & 0.59613300 & 1.52630600 & 0.13008900\end{array}$ 


$\begin{array}{crrr}\mathrm{C} & -2.48428200 & -0.08913100 & -0.78775900 \\ \mathrm{H} & -3.45138700 & -0.12394100 & -1.27413600 \\ \mathrm{H} & -2.32341300 & -2.06532300 & -0.20359200 \\ \mathrm{~N} & 1.72726800 & 0.81024200 & -0.04810900 \\ \mathrm{~N} & 0.51684500 & -1.27356100 & -0.26334600 \\ \mathrm{O} & 0.55683800 & 2.73810100 & 0.37992500 \\ \mathrm{~N} & -1.73518400 & 1.08555200 & -0.68310700 \\ \mathrm{~N} & -1.91317300 & -1.13307700 & -0.27276800 \\ \mathrm{~N} & 2.80344600 & -1.15534300 & -0.51620200 \\ \mathrm{H} & 3.66649700 & -0.62719400 & -0.55830300 \\ \mathrm{H} & 2.82861600 & -2.15523600 & -0.67819500 \\ \mathrm{O} & -0.79376100 & -0.99958700 & 1.72336900 \\ \mathrm{H} & 0.61744600 & -2.28741300 & -0.23632200 \\ \mathrm{H} & -0.03628700 & -0.62594300 & 2.20893500 \\ & & & \end{array}$

\begin{tabular}{lrrr}
\hline \multicolumn{4}{c}{ 4-OH oxidized Guanine (92) } \\
\hline SCF Done: & E(RB3LYP) $=$ & -617.780546516 a.u. \\
$0 ~ 1$ & & & \\
C & 0.62326100 & 0.73534300 & -0.04911100 \\
C & 0.63618800 & -0.70821000 & 0.38980200 \\
C & -1.58429000 & -0.60474100 & -0.26053900 \\
C & -0.65771200 & 1.47948800 & 0.14956000 \\
C & 2.44843300 & -0.05378200 & -0.82826800 \\
N & -1.75922600 & 0.73389200 & 0.03004300 \\
N & -0.47004500 & -1.35310300 & -0.20776700 \\
O & -0.62813300 & 2.70594400 & 0.40440600 \\
N & 1.93142900 & -1.09320100 & -0.25327200 \\
N & -2.71105100 & -1.23738500 & -0.62949300 \\
H & -3.57681600 & -0.72296900 & -0.70439800 \\
H & -2.69496200 & -2.22561700 & -0.83980300 \\
N & 1.64265100 & 1.08290800 & -0.77877800 \\
H & 2.38424700 & -2.00051000 & -0.15762800 \\
H & 3.40673800 & -0.06518300 & -1.33190600 \\
O & 0.74120700 & -0.86599300 & 1.78206200 \\
H & 0.34461400 & -1.72569400 & 2.00819600
\end{tabular}

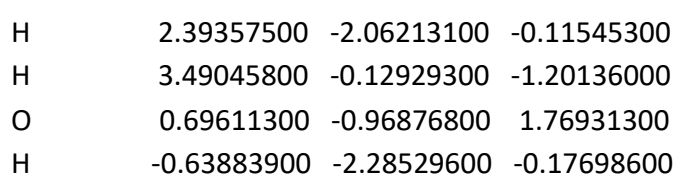

\begin{tabular}{|c|c|c|c|}
\hline \multicolumn{4}{|c|}{ 4-OH (mN1N2H) Guanine neutral (93') } \\
\hline \multicolumn{4}{|c|}{ SCF Done: $E(R B 3 L Y P)=-617.761986247$ a.u. } \\
\hline \multicolumn{4}{|c|}{01} \\
\hline C & 0.61255000 & 0.74534400 & -0.03550200 \\
\hline C & 0.59824300 & -0.72097800 & 0.34865300 \\
\hline $\mathrm{C}$ & -1.74436600 & -0.56976700 & -0.30611400 \\
\hline C & -0.66093800 & 1.49769500 & 0.12303100 \\
\hline C & 2.47777300 & -0.04388900 & -0.72056800 \\
\hline $\mathrm{N}$ & -1.77266300 & 0.78214900 & -0.03548400 \\
\hline $\mathrm{N}$ & -0.50084800 & -1.26234200 & -0.36784500 \\
\hline $\mathrm{O}$ & -0.61874100 & 2.73114700 & 0.37014200 \\
\hline $\mathrm{N}$ & 1.90459800 & -1.10751500 & -0.24156900 \\
\hline N & -2.85215200 & -1.19293700 & -0.54151100 \\
\hline $\mathrm{H}$ & -2.67855300 & -2.18208200 & -0.72946600 \\
\hline $\mathrm{N}$ & 1.70449600 & 1.10836100 & -0.64285300 \\
\hline $\mathrm{H}$ & 2.33269900 & -2.02960700 & -0.16666900 \\
\hline $\mathrm{H}$ & 3.46636000 & -0.05401000 & -1.16243300 \\
\hline $\mathrm{O}$ & 0.60733300 & -0.90105200 & 1.73569400 \\
\hline $\mathrm{H}$ & 0.45183300 & -1.84111800 & 1.93710400 \\
\hline $\mathrm{H}$ & -0.56466500 & -2.27838500 & -0.37739600 \\
\hline & 4-O-S & ip Neutral (94) & \\
\hline
\end{tabular}

$\begin{array}{lrrr}\text { SCF Done: } & E(R 3 L Y P)=-617.852398718 & \text { a.u. } \\ \text { O } & & & \\ \text { C } & 0.24804900 & -0.14212300 & -0.26144400 \\ \text { C } & 1.33167900 & -0.52886100 & 0.76542600 \\ \text { C } & -2.01444700 & -0.25557300 & -0.08712200 \\ \text { C } & -0.44017500 & 1.20774000 & 0.16674400 \\ \text { C } & 2.20288700 & -0.10212400 & -1.25831100 \\ N & -1.78085900 & 1.03346300 & 0.22899900 \\ N & -0.89704800 & -1.00454400 & -0.29218200 \\ \text { O } & 0.20006300 & 2.23804400 & 0.39188600 \\ N & 2.50057400 & -0.40462900 & 0.06537900 \\ \text { N } & -3.23241200 & -0.77009200 & -0.16955600 \\ H & -4.03429300 & -0.18428900 & 0.02166600 \\ H & -3.37381800 & -1.74677100 & -0.39433800 \\ N & 0.95247600 & 0.02273800 & -1.53814600 \\ H & 3.42798600 & -0.58113100 & 0.43727800 \\ H & 3.00664700 & -0.00273500 & -1.97824900 \\ \mathrm{O} & 1.18891700 & -0.83785600 & 1.94178600 \\ \mathrm{H} & -0.90544400 & -1.89949300 & -0.76896100\end{array}$




\begin{tabular}{|c|c|c|c|}
\hline \multicolumn{4}{|c|}{ 4-oxo 8-NHCH3-Sp Neutral (95) } \\
\hline \multicolumn{4}{|c|}{ SCF Done: $E(R 3 L Y P)=-713.748711100$ a.u. } \\
\hline 1 & & & \\
\hline C & -0.40383700 & 0.01302800 & -0.20431200 \\
\hline C & 0.24328900 & -0.81898100 & 0.92160300 \\
\hline C & -2.65363800 & -0.16999700 & -0.47299700 \\
\hline C & -1.22853900 & 1.19172800 & 0.40264500 \\
\hline C & 1.95804800 & 0.33642400 & -0.22014200 \\
\hline $\mathrm{N}$ & -2.55513100 & 0.98391800 & 0.21725400 \\
\hline N & -1.46814300 & -0.70183600 & -0.88814500 \\
\hline 0 & -0.69262000 & 2.16184000 & 0.95535500 \\
\hline N & 1.55766400 & -0.58960900 & 0.85683500 \\
\hline N & -3.81819400 & -0.73400300 & -0.76226500 \\
\hline $\mathrm{H}$ & -4.67186900 & -0.31278300 & -0.42039200 \\
\hline $\mathrm{H}$ & -3.86779900 & -1.60130000 & -1.28137000 \\
\hline N & 0.73067000 & 0.39194800 & -1.02558400 \\
\hline $\mathrm{H}$ & 2.21717300 & -0.97068100 & 1.52609800 \\
\hline 0 & -0.37332400 & -1.54294900 & 1.71349800 \\
\hline $\mathrm{H}$ & -1.38917600 & -1.69882400 & -1.06613000 \\
\hline N & 3.08725900 & -0.07385500 & -1.00654500 \\
\hline $\mathrm{H}$ & 2.92098100 & -1.01437500 & -1.36269800 \\
\hline C & 4.35877400 & -0.03291400 & -0.26636900 \\
\hline $\mathrm{H}$ & 4.39346700 & -0.70954400 & 0.59968800 \\
\hline $\mathrm{H}$ & 5.17485200 & -0.30578400 & -0.93871400 \\
\hline $\mathrm{H}$ & 4.53922600 & 0.98572500 & 0.08863800 \\
\hline $\mathrm{H}$ & 2.20795100 & 1.30412100 & 0.22629900 \\
\hline $\mathrm{H}$ & 0.61928700 & 1.32065800 & -1.42565400 \\
\hline
\end{tabular}

$\begin{array}{lrrr}\mathrm{C} & 4.30464800 & 0.13062500 & -0.33460500 \\ \mathrm{H} & 4.38862700 & -0.51233600 & 0.55302300 \\ \mathrm{H} & 5.12960000 & -0.11482300 & -1.00653900 \\ \mathrm{H} & 4.42106500 & 1.17098500 & -0.01871300 \\ \mathrm{H} & 2.04787900 & 1.32846300 & 0.13612300\end{array}$

\begin{tabular}{|c|c|c|c|}
\hline \multicolumn{4}{|c|}{ 4-oxo-8-NHCH $3-S p$ Radical Neutral (97) } \\
\hline \multicolumn{4}{|c|}{ SCF Done: $E(U 3 L Y P)=-713.092074836$ a.u. } \\
\hline \multicolumn{4}{|c|}{02} \\
\hline C & 0.37711700 & 0.16801400 & 0.02110100 \\
\hline C & -0.30289800 & -0.37782800 & -1.24221400 \\
\hline C & 2.51032100 & -0.44131500 & 0.57300100 \\
\hline C & 1.45933700 & 1.25068200 & -0.26307100 \\
\hline C & -1.92341800 & 0.10971100 & 0.30944600 \\
\hline $\mathrm{N}$ & 2.69290500 & 0.79556800 & 0.06838000 \\
\hline $\mathrm{N}$ & 1.21227900 & -0.81762500 & 0.74317900 \\
\hline $\mathrm{O}$ & 1.18566300 & 2.36046900 & -0.74120600 \\
\hline $\mathrm{N}$ & -1.68416000 & -0.53875000 & -0.83081900 \\
\hline $\mathrm{N}$ & 3.52479900 & -1.22708900 & 0.91475800 \\
\hline $\mathrm{H}$ & 4.47247200 & -0.91706900 & 0.74566400 \\
\hline $\mathrm{H}$ & 3.36831200 & -2.15723800 & 1.28026500 \\
\hline $\mathrm{N}$ & -0.76146300 & 0.64845700 & 0.78361300 \\
\hline $\mathrm{H}$ & -2.41067600 & -0.87328000 & -1.45277900 \\
\hline $\mathrm{O}$ & 0.25484700 & -1.18885400 & -2.07515800 \\
\hline $\mathrm{H}$ & 0.97379100 & -1.80443000 & 0.69948500 \\
\hline N & -3.11749200 & 0.31796700 & 0.87156000 \\
\hline $\mathrm{H}$ & -3.11386300 & 0.72175700 & 1.80078700 \\
\hline C & -4.33123500 & -0.36033500 & 0.41936900 \\
\hline $\mathrm{H}$ & -4.25549700 & -1.44609000 & 0.54222600 \\
\hline $\mathrm{H}$ & -5.16323200 & 0.00900500 & 1.01741000 \\
\hline $\mathrm{H}$ & -4.52988800 & -0.12684100 & -0.63000900 \\
\hline $\mathrm{H}$ & -0.66890800 & 0.87799800 & 1.76737200 \\
\hline
\end{tabular}




$\begin{array}{lrrr}\mathrm{N} & -0.62222600 & -1.03339000 & 0.31270900 \\ \mathrm{H} & -2.49428600 & 1.37012400 & -0.79290700 \\ \mathrm{O} & 0.04340900 & 2.14035900 & -1.21182100 \\ \mathrm{H} & 1.25762300 & -1.22848200 & -1.67371400 \\ \mathrm{~N} & -2.97684700 & -1.00252800 & 0.41317600 \\ \mathrm{H} & -2.93075700 & -1.95926600 & 0.74318300 \\ \mathrm{C} & -4.25588400 & -0.55462100 & -0.14155900 \\ \mathrm{H} & -4.32618200 & -0.73397900 & -1.21980200 \\ \mathrm{H} & -5.04797600 & -1.11124800 & 0.35892700 \\ \mathrm{H} & -4.41528000 & 0.50753100 & 0.06042100\end{array}$

4-oxo-8-OH-Sp Neutral (99)

SCF Done: $E(R 3 L Y P)=-694.308952624$ a.u.

01

$\begin{array}{llll}\text { C } & -0.01140300 & 0.02498400 & -0.20806400\end{array}$

$\begin{array}{llll}\text { C } & 0.67056100 & -0.56113300 & 1.04355700\end{array}$

$\begin{array}{llll}\text { C } & -2.25123700 & -0.31379000 & -0.39960300\end{array}$

$\begin{array}{lllll}\text { C } & -0.89094100 & 1.25539200 & 0.18569300\end{array}$

$\begin{array}{lllll}\text { C } & 2.35701400 & 0.24651700 & -0.37778400\end{array}$

$\begin{array}{lllll}\mathrm{N} & -2.20450700 & 0.94962200 & 0.06880100\end{array}$

N $\quad-1.04418100 \quad-0.85452000 \quad-0.73291600$

$\begin{array}{lllll}\mathrm{O} & -0.39872900 & 2.33370300 & 0.54060300\end{array}$

$\begin{array}{lllll}\mathrm{N} & 1.98388200 & -0.33639000 & 0.90163400\end{array}$

$\begin{array}{lllll}\mathrm{N} & -3.38993600 & -0.97134300 & -0.56645200\end{array}$

H $\quad-4.25938200-0.53124500-0.29560900$

H $\quad-3.40298200 \quad-1.92023100 \quad-0.91766200$

$\begin{array}{lllll}\mathrm{N} & 1.09431900 & 0.32324800 & -1.09493600\end{array}$

H $\quad 2.67870600 \quad-0.64922300 \quad 1.57131000$

$\begin{array}{lllll}\mathrm{O} & 0.08401300 & -1.11101700 & 1.97987600\end{array}$

H $\quad-0.91776100-1.86250100-0.73384200$

$\begin{array}{lllll}\text { O } & 3.26510200 & -0.58379000 & -1.07745800\end{array}$

H $\quad 4.16820600 \quad-0.28837200 \quad-0.88171400$

$\begin{array}{lllll}H & 0.99142400 & 1.22736200 & -1.54678100\end{array}$

$\begin{array}{lllll}H & 2.81769000 & 1.22691400 & -0.23558300\end{array}$

$\begin{array}{llll}\mathrm{H} & -4.29082700 & 0.33099900 & -0.05690000 \\ \mathrm{H} & -3.82039000 & -0.93637100 & -1.16111900 \\ \mathrm{~N} & 0.93199500 & 0.30545000 & -1.29333900 \\ \mathrm{H} & 2.75294900 & -1.55607200 & 0.71237300 \\ \mathrm{O} & 0.18762300 & -1.93483400 & 1.37135100 \\ \mathrm{H} & -1.38795400 & -1.47264600 & -1.33428800 \\ \mathrm{O} & 2.94293800 & 1.04948500 & -0.28443800 \\ \mathrm{H} & 3.47770500 & 1.43458600 & -0.99672300 \\ \mathrm{H} & 2.83477100 & -0.52240000 & -1.60911800\end{array}$

\section{4-oxo-8-OH-Sp Radical Neutral (101)}

SCF Done: $E(U 3 L Y P)=-693.645442974$ a.u.

02

C $\quad-0.00642200 \quad-0.06804000 \quad-0.16272700$

$\begin{array}{lllll}\text { C } & -0.70293200 & 0.75331000 & 0.94831500\end{array}$

$\begin{array}{lllll}\text { C } & 2.23504500 & 0.21148900 & -0.46034100\end{array}$

C $\quad 0.87667600 \quad-1.21864900 \quad 0.42134200$

$\begin{array}{lllll}C & -2.26746500 & 0.24702900 & -0.66243400\end{array}$

N $\quad 2.18997200 \quad-0.95284200 \quad 0.21457200$

N $\quad \begin{array}{llll}\text { N } & 1.02305600 & 0.70872200 & -0.84486300\end{array}$

$\begin{array}{lllll}\mathrm{O} & 0.39503600 & -2.20397600 & 0.99115400\end{array}$

$\begin{array}{lllll}\mathrm{N} & -2.00513500 & 0.83536700 & 0.59529200\end{array}$

N $\quad 3.37024100 \quad 0.82499500 \quad-0.76270300$

H $\quad 4.24767600 \quad 0.42674200 \quad-0.45474100$

H $\quad 3.37561600 \quad 1.69986500 \quad-1.27154200$

N $\quad-1.12270300 \quad-0.50391200-0.96960800$

$\mathrm{H} \quad-2.69070300 \quad 1.37806800 \quad 1.11095400$

$\begin{array}{lllll}0 & -0.14164600 & 1.27445400 & 1.91665000\end{array}$

H $\quad 0.90380000 \quad 1.70332600 \quad-1.01495100$

$\begin{array}{lllll}0 & -3.46798400 & -0.40961300 & -0.85901000\end{array}$

H $\quad-4.17920200 \quad 0.24669900 \quad-0.94148800$

H $\quad-0.93785900 \quad-0.67876400 \quad-1.95234500$

\section{4-oxo-8-OH-Sp Neutral (102)}

SCF Done: $E(R 3 L Y P)=-693.094586523$ a.u.

01

$\begin{array}{llll}\text { C } & 0.00841400 & -0.01151100 & 0.21927500\end{array}$

$\begin{array}{lllll}\text { C } & 0.72586000 & 1.01351700 & -0.69312300\end{array}$

$\begin{array}{lllll}\text { C } & -2.25435500 & 0.03784400 & 0.43269900\end{array}$

C $\quad-0.75377400 \quad-1.05185300-0.67880900$

$\begin{array}{lllll}\mathrm{C} & 2.16293700 & -0.16216200 & 0.55928300\end{array}$

N $\quad-2.08889600 \quad-0.93424200-0.48498700$

N $\quad \begin{array}{llll}\text { N } & -1.10024500 & 0.53519300 & 0.95891400\end{array}$

$\begin{array}{lllll}\mathrm{N} & -0.16813800 & -1.83205400 & -1.43532900\end{array}$

N $\quad \begin{array}{lllll}2.05473100 & 0.79164700 & -0.45477800\end{array}$

N $\quad \begin{array}{llll}-3.44507000 & 0.46710200 & 0.82409100\end{array}$

H $\quad-4.27840800 \quad 0.06872800 \quad 0.41215200$

H $\quad-3.53545100 \quad 1.19116700 \quad 1.52534100$

$\begin{array}{llll}\mathrm{N} & 1.05609600 & -0.62944800 & 1.01846400\end{array}$ 


$\begin{array}{lrrr}H & 2.82474500 & 1.29689300 & -0.88450200 \\ \mathrm{O} & 0.22816000 & 1.81715500 & -1.46802600 \\ \mathrm{H} & -1.05980600 & 1.44656500 & 1.40350100 \\ \mathrm{O} & 3.35159200 & -0.54783700 & 1.01369000 \\ \mathrm{H} & 4.08520700 & -0.06824300 & 0.59294300\end{array}$

\begin{tabular}{|c|c|c|c|}
\hline \multicolumn{4}{|c|}{ 4,8-oxoSp Neutral (103) } \\
\hline \multicolumn{4}{|c|}{ SCF Done: $E(R 3 L Y P)=-693.121945757$ a.u. } \\
\hline C & -0.01387600 & -0.00585700 & -0.22409500 \\
\hline C & -0.75019400 & 1.05937600 & 0.62106200 \\
\hline C & 2.24811100 & 0.01667700 & -0.39005100 \\
\hline C & 0.71653100 & -1.04419200 & 0.70497200 \\
\hline C & -2.31561600 & -0.15794000 & -0.53719100 \\
\hline $\mathrm{N}$ & 2.05516900 & -0.93523700 & 0.54256300 \\
\hline $\mathrm{N}$ & 1.10842400 & 0.50478600 & -0.96709300 \\
\hline 0 & 0.09784700 & -1.81484500 & 1.44226700 \\
\hline $\mathrm{N}$ & -2.08067100 & 0.85270500 & 0.40599700 \\
\hline $\mathrm{N}$ & 3.44687000 & 0.43738200 & -0.75824000 \\
\hline $\mathrm{H}$ & 4.26948200 & 0.04913400 & -0.31532100 \\
\hline $\mathrm{H}$ & 3.55579600 & 1.14468700 & -1.47433400 \\
\hline $\mathrm{N}$ & -1.09726600 & -0.56698600 & -1.00076000 \\
\hline $\mathrm{H}$ & -2.82835800 & 1.39858300 & 0.82387200 \\
\hline $\mathrm{O}$ & -0.22632000 & 1.90075000 & 1.33422600 \\
\hline $\mathrm{H}$ & 1.08979600 & 1.42914400 & -1.38822800 \\
\hline $\mathrm{O}$ & -3.42278900 & -0.56137100 & -0.87699600 \\
\hline $\mathrm{H}$ & -1.01403400 & -1.47476900 & -1.44741300 \\
\hline
\end{tabular}




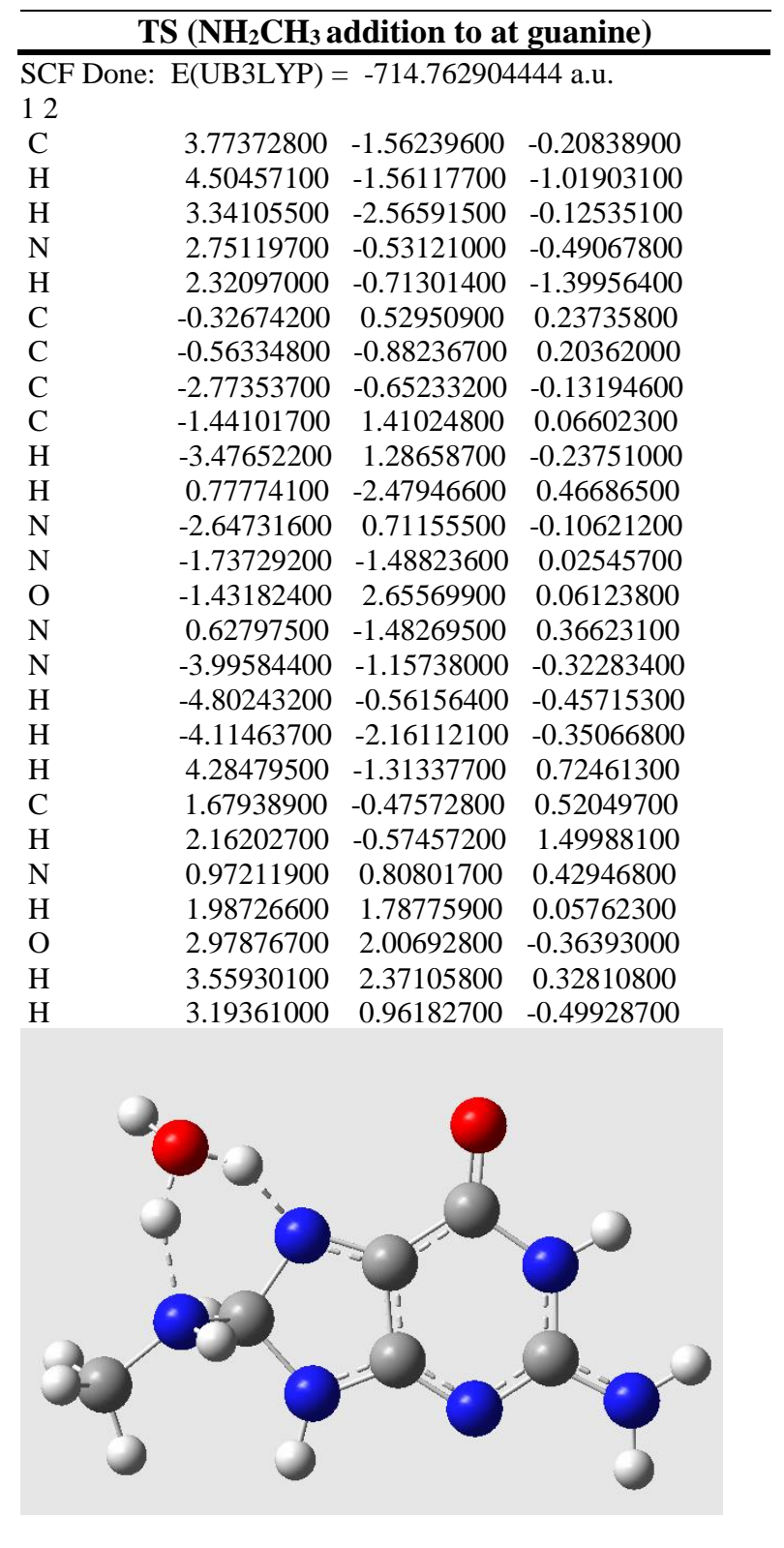

\begin{tabular}{llll}
\hline \multicolumn{5}{c}{ TS (Guanine radical $+\mathrm{NH}_{2} \mathrm{CH}_{3}$ at C8) } \\
\hline SCF Done: & E(U3LYP) $=$ & -714.301195379 & a.u. \\
$\mathrm{O} 2$ & & & \\
$\mathrm{C}$ & -2.42984200 & -1.23059700 & 1.49665800 \\
$\mathrm{H}$ & -3.33325300 & -1.15070200 & 2.10199400 \\
$\mathrm{H}$ & -1.67764800 & -0.53730700 & 1.87270100 \\
$\mathrm{~N}$ & -2.79450100 & -0.85369200 & 0.10105300 \\
$\mathrm{H}$ & -3.46696300 & -1.52525100 & -0.27801700 \\
$\mathrm{C}$ & 0.21307200 & 0.49092100 & -0.33331900 \\
$\mathrm{C}$ & 0.58251100 & -0.88386300 & -0.37651700 \\
$\mathrm{C}$ & 2.69345200 & -0.35824100 & 0.15330700 \\
$\mathrm{C}$ & 1.22182000 & 1.46261100 & 0.01944100 \\
$\mathrm{H}$ & -0.53639500 & -2.54466400 & -1.04692900 \\
$\mathrm{~N}$ & 2.47095400 & 0.96241700 & 0.25856300 \\
$\mathrm{~N}$ & 1.79154000 & -1.35199500 & -0.15184100 \\
$\mathrm{O}$ & 0.96644000 & 2.69938100 & 0.10010500 \\
$\mathrm{~N}$ & -0.54152600 & -1.59920400 & -0.68348400 \\
$\mathrm{~N}$ & 3.95307700 & -0.78726900 & 0.37279500 \\
$\mathrm{H}$ & 4.67111100 & -0.12854100 & 0.63754400 \\
$\mathrm{H}$ & 4.16545200 & -1.77380300 & 0.33866800 \\
$\mathrm{H}$ & -2.05375700 & -2.25159800 & 1.52626000 \\
$\mathrm{C}$ & -1.64308600 & -0.69827400 & -0.88948200 \\
$\mathrm{H}$ & -2.10261200 & -0.81630400 & -1.87431700 \\
$\mathrm{~N}$ & -1.08084800 & 0.63194700 & -0.69565800 \\
$\mathrm{H}$ & -3.22087500 & 0.15693300 & 0.12780300 \\
$\mathrm{O}$ & -3.19176900 & 1.72829800 & 0.04556500 \\
$\mathrm{H}$ & -1.88190300 & 1.36758900 & -0.35257100 \\
$\mathrm{H}$ & -3.17896800 & 2.07144800 & 0.95099200 \\
& & & \\
& & & \\
& & & \\
& & &
\end{tabular}

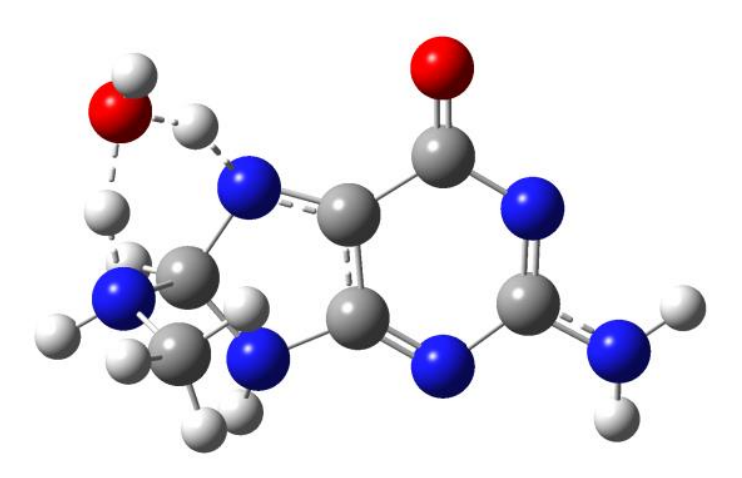




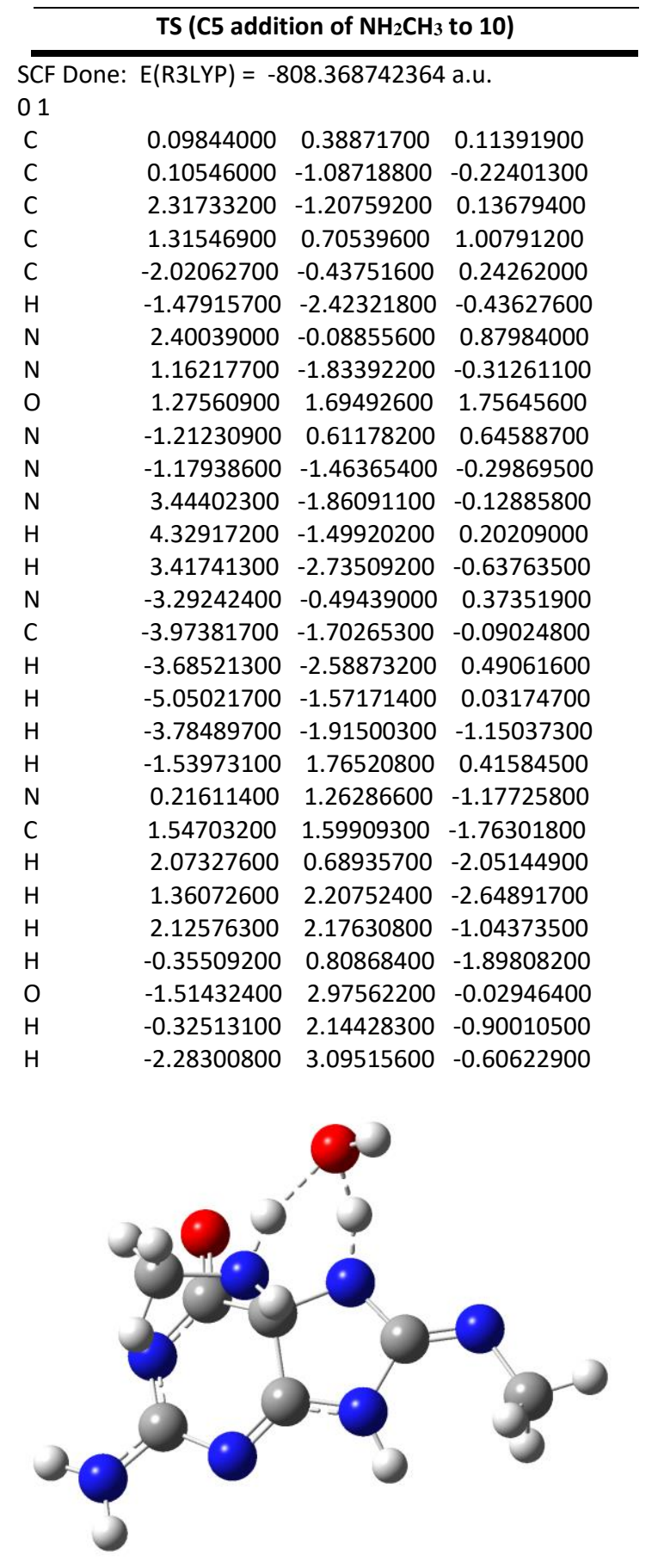




\begin{tabular}{|c|c|c|c|}
\hline \multicolumn{4}{|c|}{ TS (Acyl Group Migration (11AN $\rightarrow$ 12AN) } \\
\hline \multicolumn{4}{|c|}{ SCF Done: $E(R 3 L Y P)=-731.424705424$ a.u. } \\
\hline C & 0.11499900 & 0.78764500 & -0.52010400 \\
\hline C & 0.05984700 & -0.65012200 & -0.65477300 \\
\hline C & 2.11348700 & -1.26037500 & -0.01190800 \\
\hline C & 0.93520500 & 0.32086800 & 1.11996100 \\
\hline C & -2.05109900 & 0.02557200 & -0.15920700 \\
\hline $\mathrm{N}$ & 2.04642200 & -0.42138400 & 1.06268900 \\
\hline $\mathrm{N}$ & 1.09243600 & -1.48881900 & -0.84800000 \\
\hline 0 & 0.58193700 & 1.08782600 & 2.02863400 \\
\hline $\mathrm{N}$ & -1.25039000 & -1.05778400 & -0.55777900 \\
\hline $\mathrm{N}$ & 3.27649300 & -1.92461300 & -0.20120700 \\
\hline $\mathrm{H}$ & 3.95518900 & -1.91654400 & 0.54955100 \\
\hline H & 3.28192100 & -2.71622800 & -0.83198800 \\
\hline $\mathrm{N}$ & -1.25872800 & 1.15084700 & -0.28700200 \\
\hline H & -1.48912500 & -2.00619100 & -0.28351200 \\
\hline $\mathrm{N}$ & -3.27327900 & 0.01076900 & 0.24070500 \\
\hline C & -3.93467900 & -1.29638500 & 0.26816300 \\
\hline $\mathrm{H}$ & -4.07041200 & -1.71190600 & -0.73896200 \\
\hline $\mathrm{H}$ & -3.38286400 & -2.03611100 & 0.86426300 \\
\hline $\mathrm{H}$ & -4.92451200 & -1.18419000 & 0.71500500 \\
\hline $\mathrm{N}$ & 1.02300600 & 1.61308800 & -1.08483700 \\
\hline C & 0.94176200 & 2.99484500 & -0.62526200 \\
\hline $\mathrm{H}$ & 1.61917800 & 3.61087800 & -1.22515700 \\
\hline $\mathrm{H}$ & -0.06104000 & 3.44566800 & -0.71674600 \\
\hline $\mathrm{H}$ & 1.24827000 & 3.12120200 & 0.42939900 \\
\hline $\mathrm{H}$ & -1.50095200 & 1.98380100 & 0.23586400 \\
\hline
\end{tabular}

\begin{tabular}{lrrr}
\hline \multicolumn{4}{c}{ TS (Acyl Group Migration (11AN' $\rightarrow \mathbf{1 2 A N ^ { \prime } )}$} \\
SCF Done: E(R3LYP) $=-731.446469687$ & a.u. \\
-1 1 & & & \\
$\mathrm{C}$ & -0.00916000 & 0.79355400 & -0.48756200 \\
$\mathrm{C}$ & -0.00687800 & -0.63666900 & -0.61067400 \\
$\mathrm{C}$ & 2.06039000 & -1.29677400 & -0.04364300 \\
$\mathrm{C}$ & 0.93063500 & 0.24951900 & 1.18444800 \\
$\mathrm{C}$ & -2.07612800 & 0.13687000 & -0.13577600 \\
$\mathrm{~N}$ & 2.02006200 & -0.49892100 & 1.07803900 \\
$\mathrm{~N}$ & 1.03018800 & -1.47048800 & -0.87011400 \\
$\mathrm{O}$ & 0.55785700 & 0.95822000 & 2.12165900 \\
$\mathrm{~N}$ & -1.30161500 & -1.01937200 & -0.43980300 \\
$\mathrm{~N}$ & 3.21170600 & -1.96251200 & -0.26438800 \\
$\mathrm{H}$ & 3.91087100 & -1.97733700 & 0.46650600 \\
$\mathrm{H}$ & 3.22777500 & -2.69642000 & -0.96120200 \\
$\mathrm{~N}$ & -1.28698000 & 1.24647400 & -0.23654100 \\
$\mathrm{H}$ & -1.57203000 & -1.96177600 & -0.17924000 \\
$\mathrm{~N}$ & -3.34176600 & 0.12164600 & 0.18704500 \\
$\mathrm{C}$ & -3.99053300 & -1.18888200 & 0.17494700 \\
$\mathrm{H}$ & -3.96092300 & -1.67442600 & -0.81206900 \\
$\mathrm{H}$ & -3.54953800 & -1.89434300 & 0.89651000 \\
$\mathrm{H}$ & -5.04309200 & -1.07226500 & 0.44481100 \\
$\mathrm{~N}$ & 0.92319100 & 1.63560800 & -1.12337300 \\
$\mathrm{C}$ & 1.14409800 & 2.96565200 & -0.53484800 \\
$\mathrm{H}$ & 1.85433500 & 3.50173800 & -1.16781400 \\
$\mathrm{H}$ & 0.20956200 & 3.52871500 & -0.51619500 \\
$\mathrm{H}$ & 1.54791000 & 2.91824000 & 0.48532400 \\
$\mathrm{H}$ & 1.81424800 & 1.15545500 & -1.22730700 \\
& & & \\
\hline & & & \\
\hline & & & \\
& & &
\end{tabular}
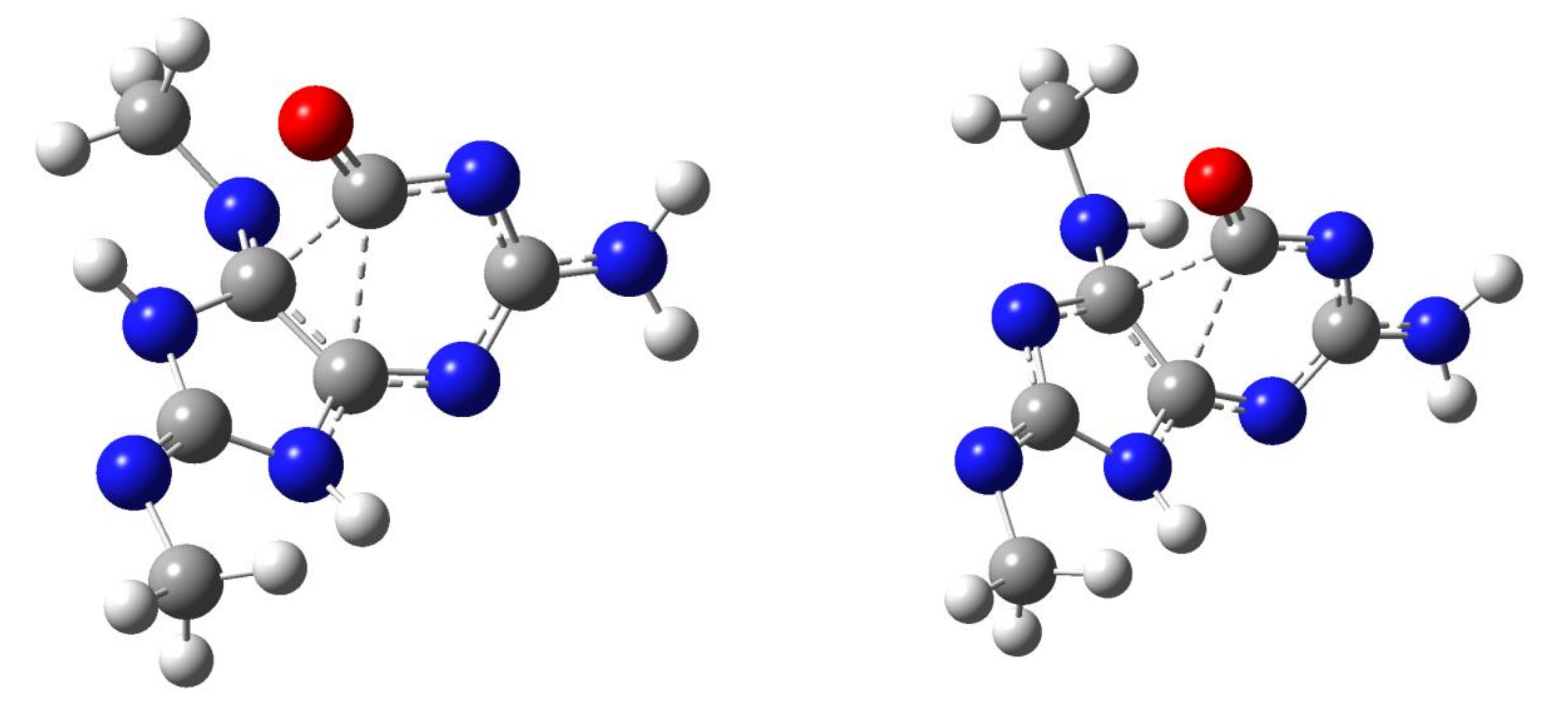


\begin{tabular}{|c|c|c|c|}
\hline \multicolumn{4}{|c|}{ TS (C5 addition of $\mathrm{H}_{2} \mathrm{O}$ to 10 ) } \\
\hline \multicolumn{4}{|c|}{$\begin{array}{l}\text { SCF Done: } E(R 3 L Y P)=-788.911187173 \text { a.u. } \\
01\end{array}$} \\
\hline C & -0.14438400 & 0.46626000 & -0.11942900 \\
\hline C & -0.21401100 & -1.00378700 & 0.17249600 \\
\hline C & -2.45256700 & -0.95257900 & 0.06533900 \\
\hline C & -1.42921900 & 0.98030900 & -0.75280100 \\
\hline C & 1.89197000 & -0.37318700 & -0.33636100 \\
\hline $\mathrm{H}$ & 1.34545500 & -2.39209600 & 0.28344300 \\
\hline $\mathrm{N}$ & -2.54906300 & 0.24948800 & -0.53424800 \\
\hline $\mathrm{N}$ & -1.30594300 & -1.68791800 & 0.33826800 \\
\hline $\mathrm{O}$ & -1.43155100 & 2.04636900 & -1.39406200 \\
\hline $\mathrm{N}$ & 1.10234000 & 0.72772700 & -0.63489200 \\
\hline $\mathrm{N}$ & 1.05566800 & -1.42767300 & 0.16714600 \\
\hline $\mathrm{N}$ & -3.58910700 & -1.57413000 & 0.37623400 \\
\hline $\mathrm{H}$ & -4.47762600 & -1.12763800 & 0.19236000 \\
\hline $\mathrm{H}$ & -3.56781400 & -2.49659100 & 0.79040500 \\
\hline $\mathrm{N}$ & 3.15886300 & -0.44769600 & -0.51350000 \\
\hline C & 3.83100900 & -1.69696100 & -0.15818000 \\
\hline $\mathrm{H}$ & 3.55962800 & -2.51628800 & -0.83663800 \\
\hline $\mathrm{H}$ & 4.91009800 & -1.55476000 & -0.23693200 \\
\hline $\mathrm{H}$ & 3.61523900 & -2.02135700 & 0.86768400 \\
\hline $\mathrm{H}$ & 1.55035700 & 2.17831600 & 0.07238900 \\
\hline $\mathrm{O}$ & 1.34651000 & 2.83916000 & 0.83894700 \\
\hline $\mathrm{H}$ & 0.54140500 & 2.13530300 & 1.29348300 \\
\hline $\mathrm{H}$ & 2.12815600 & 2.88437000 & 1.41647100 \\
\hline $\mathrm{O}$ & -0.22460500 & 1.12988600 & 1.41595400 \\
\hline $\mathrm{H}$ & -1.13382300 & 1.38850200 & 1.65118400 \\
\hline
\end{tabular}

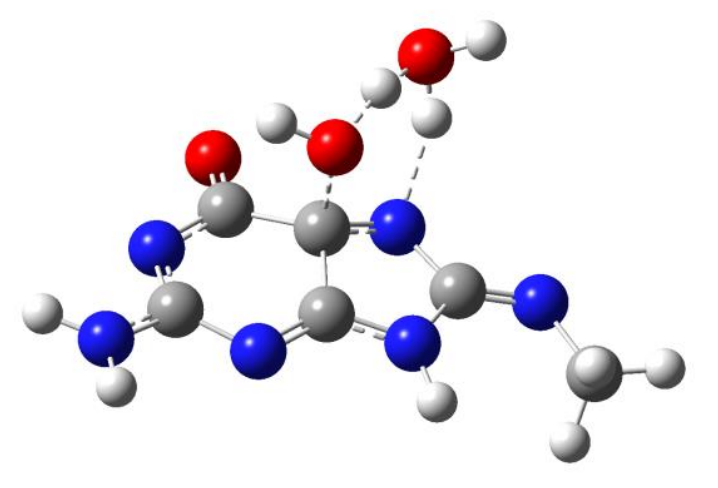

TS (Acyl Group Migration, 15 $\rightarrow$ 16)

SCF Done: $E(R 3 L Y P)=-712.460426100$ a.u.

01

$\begin{array}{llll}\text { C } & -0.00596500 & 0.91245000 & -0.78136400\end{array}$

C $\quad 0.16161700 \quad-0.47267300 \quad-0.63674500$

$\begin{array}{llll}\text { C } & 2.27248500 & -0.83913700 & 0.05660300\end{array}$

C $\quad 1.07256300 \quad 0.82737400 \quad 1.09966500$

$\begin{array}{lllll}\text { C } & -2.02575200 & 0.04105500 & -0.20936100\end{array}$

N $\quad \begin{array}{llll}\mathrm{N} & 2.15384600 & 0.11963500 & 1.04675200\end{array}$

N $\quad 1.28925800 \quad-1.20791600 \quad-0.75356200$

$\begin{array}{lllll}\mathrm{O} & 0.56440300 & 1.66869900 & 1.80392500\end{array}$

N $\quad-1.08890700-0.99309900-0.34543600$

N $\quad 3.46529800 \quad-1.44558500 \quad-0.01465300$

H $\quad 4.19547300 \quad-1.20616300 \quad 0.64047800$

$\mathrm{H} \quad 3.60492100 \quad-2.20567700 \quad-0.66585700$

N $\quad-1.35270600 \quad 1.19427200 \quad-0.61799400$

H $\quad-1.20514700 \quad-1.88827600 \quad 0.11712700$

N $\quad \begin{array}{llll}-3.24244900 & 0.00248900 & 0.18998200\end{array}$

O $\quad 0.73725800 \quad 1.84080300 \quad-1.43671100$

$\begin{array}{llll}\text { C } & -3.76259000 & -1.31234100 & 0.56043500\end{array}$

H $\quad-3.16149900-1.80077800 \quad 1.34018300$

H $\quad-4.77520300-1.198552000 .95117500$

H $\quad-3.81486400-1.99361800 \quad-0.29927200$

H $\quad-1.69035800 \quad 2.11632200 \quad-0.36452100$

H $\quad 1.58886100 \quad 1.47179000 \quad-1.72805100$

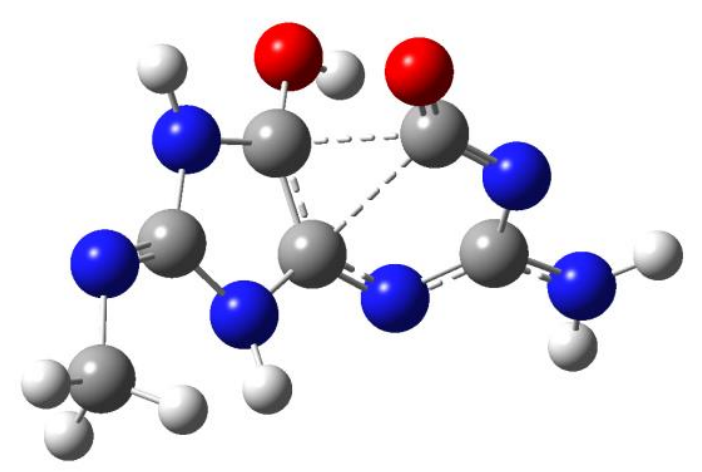




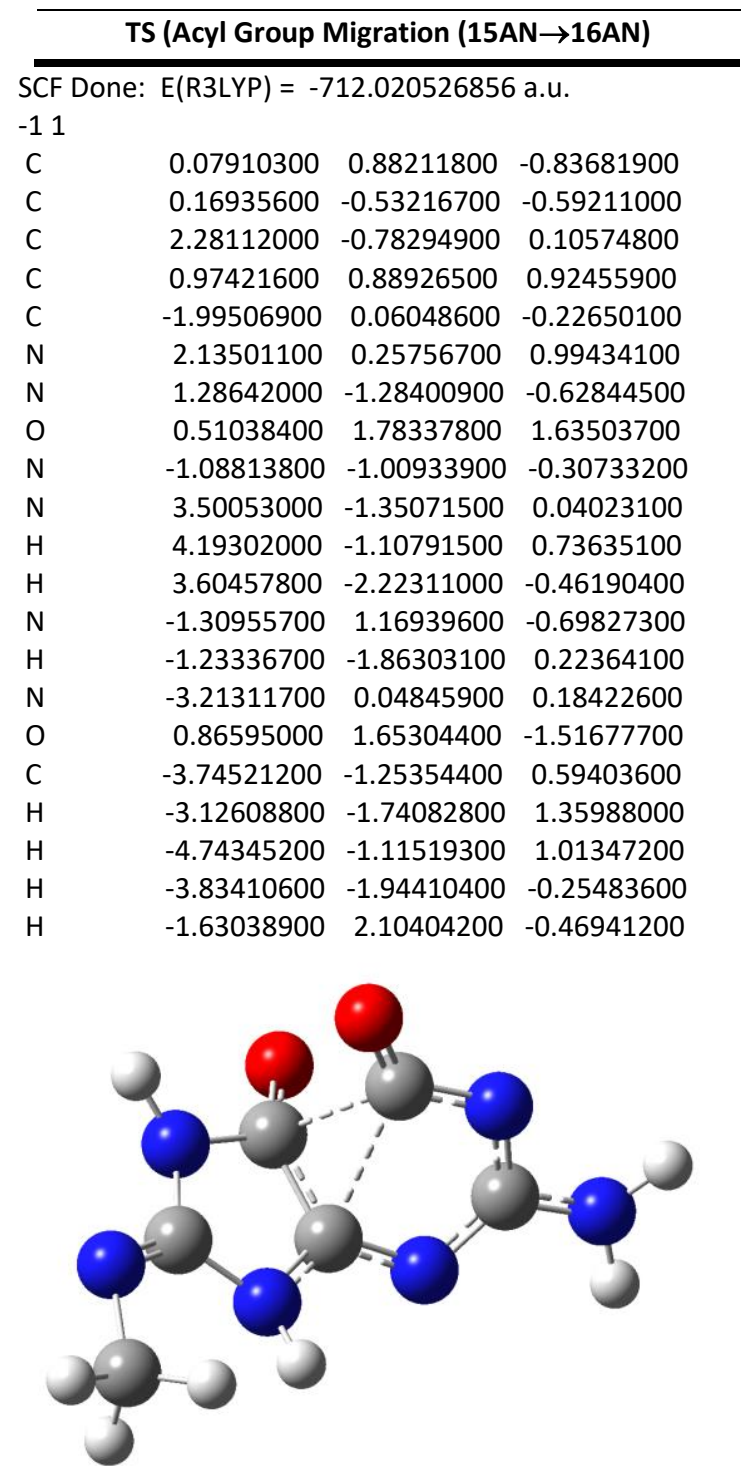




\section{TS $\left(\mathrm{H}_{2} \mathrm{O}\right.$ addition to Guanine at $\left.\mathrm{C8}\right)$}

\begin{tabular}{lrcc}
\hline SCF Done: & E(UB3LYP) $=$ & -695.313778276 a.u. \\
12 & & & \\
$\mathrm{C}$ & -0.03272500 & 0.29379800 & -0.40628000 \\
$\mathrm{C}$ & 0.44975300 & -1.03593200 & -0.18384300 \\
$\mathrm{C}$ & 2.56495800 & -0.38069900 & 0.18228100 \\
$\mathrm{C}$ & 0.90599400 & 1.37133300 & -0.31909800 \\
$\mathrm{H}$ & -0.59033800 & -2.86340600 & -0.32351900 \\
$\mathrm{~N}$ & 2.20320200 & 0.92575700 & -0.01174200 \\
$\mathrm{~N}$ & 1.69759600 & -1.40062800 & 0.10583100 \\
$\mathrm{O}$ & 0.68897100 & 2.58812200 & -0.48240900 \\
$\mathrm{~N}$ & -0.61327300 & -1.85123600 & -0.32152100 \\
$\mathrm{~N}$ & 3.84559900 & -0.63555600 & 0.46964800 \\
$\mathrm{H}$ & 4.53828400 & 0.09987600 & 0.51803900 \\
$\mathrm{H}$ & 4.13568400 & -1.59499500 & 0.60121300 \\
$\mathrm{C}$ & -1.78820400 & -1.05387600 & -0.62717700 \\
$\mathrm{H}$ & -2.26367100 & -1.37649800 & -1.55717600 \\
$\mathrm{~N}$ & -1.34650900 & 0.32541600 & -0.68182700 \\
$\mathrm{H}$ & -2.93037400 & 1.53937200 & 0.24944300 \\
$\mathrm{O}$ & -3.66659500 & 1.11689000 & 0.75090500 \\
$\mathrm{H}$ & -3.63244800 & 1.43843800 & 1.67206300 \\
$\mathrm{H}$ & -3.40454700 & 0.08801600 & 0.70142900 \\
$\mathrm{O}$ & -2.78935200 & -1.18044900 & 0.42545700 \\
$\mathrm{H}$ & -3.44178500 & -1.85734500 & 0.17416200 \\
$\mathrm{H}$ & 2.91409300 & 1.64981500 & 0.05882400
\end{tabular}

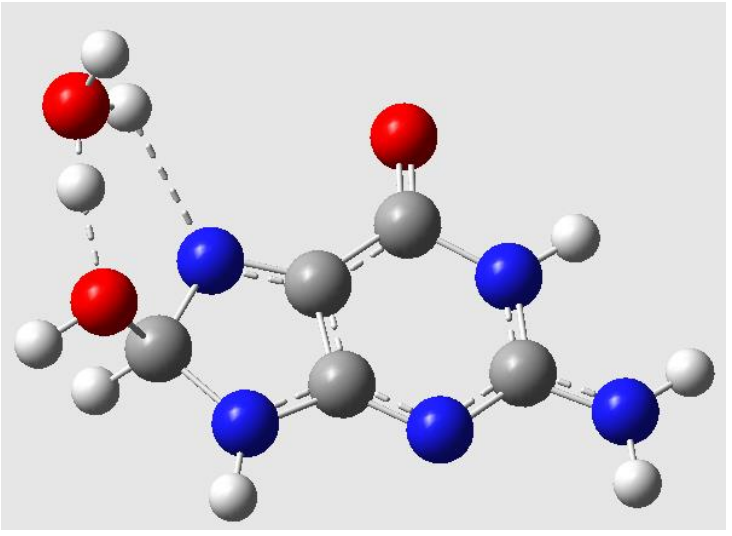

TS (Guanine radical $+\mathrm{H}_{2} \mathrm{O}$ at $\mathrm{C} 8$ )

SCF Done: $\mathrm{E}(\mathrm{U}$ LYYP) $=-694.839942476$

02

$\begin{array}{llll}\text { C } & 0.51371500 & 0.33935400 & -0.50649700\end{array}$

$\begin{array}{llll}\text { C } & 1.03529700 & -0.94569400 & -0.79011100\end{array}$

$\begin{array}{llll}\text { C } & 3.03982300 & -0.31817900 & -0.01651200\end{array}$

$\begin{array}{llll}\text { C } & 1.38958600 & 1.34217800 & 0.08984000\end{array}$

H $\quad 0.00253000 \quad-2.62938700 \quad-1.65533600$

$\begin{array}{llll}\mathrm{N} & 2.66875700 & 0.92771500 & 0.30620600\end{array}$

N $\quad 2.26321200 \quad-1.33561900 \quad-0.57314600$

$\begin{array}{lllll}0 & 0.97845100 & 2.48929400 & 0.37051600\end{array}$

N $\quad-0.02504600 \quad-1.66982900 \quad-1.32015500$

$\begin{array}{lllll}N & 4.30677700 & -0.67808600 & 0.20848500\end{array}$

$\begin{array}{llll}H & 4.95840300 & -0.01615300 & 0.61041000\end{array}$

H $\quad 4.61994100-1.61166900-0.02469800$

C $\quad-1.11017500-0.88590900-1.33596800$

H $\quad-2.05340500 \quad-1.1549650000-1.77879000$

$\begin{array}{llll}N & -0.78835600 & 0.34630000 & -0.87704700\end{array}$

$\begin{array}{lllll}\mathrm{H} & -3.05689800 & 0.32071600 & 0.35088200\end{array}$

$\begin{array}{lllll}0 & -3.04015600 & 1.34541900 & 0.07909400\end{array}$

$\begin{array}{llll}\mathrm{H} & -1.55380200 & 1.02286200 & -0.58859900\end{array}$

$\begin{array}{llll}H & -2.93834400 & 1.85092800 & 0.89855900\end{array}$

$\begin{array}{lllll}0 & -2.83949200 & -1.10417400 & 0.58433700\end{array}$

$\begin{array}{lllll}\text { H } & -3.51007700 & -1.61535600 & 0.10724900\end{array}$

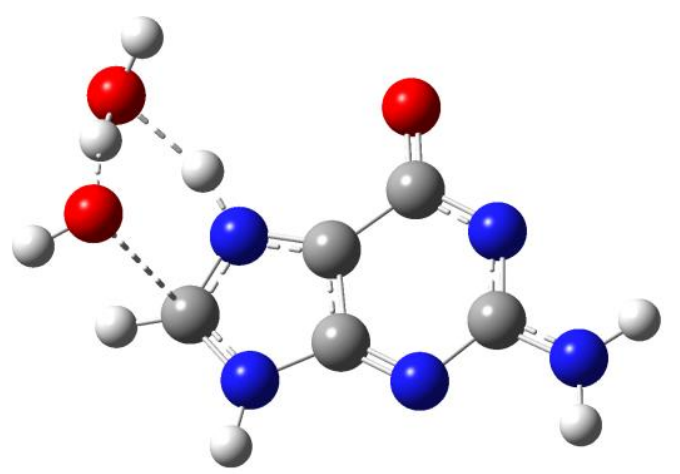




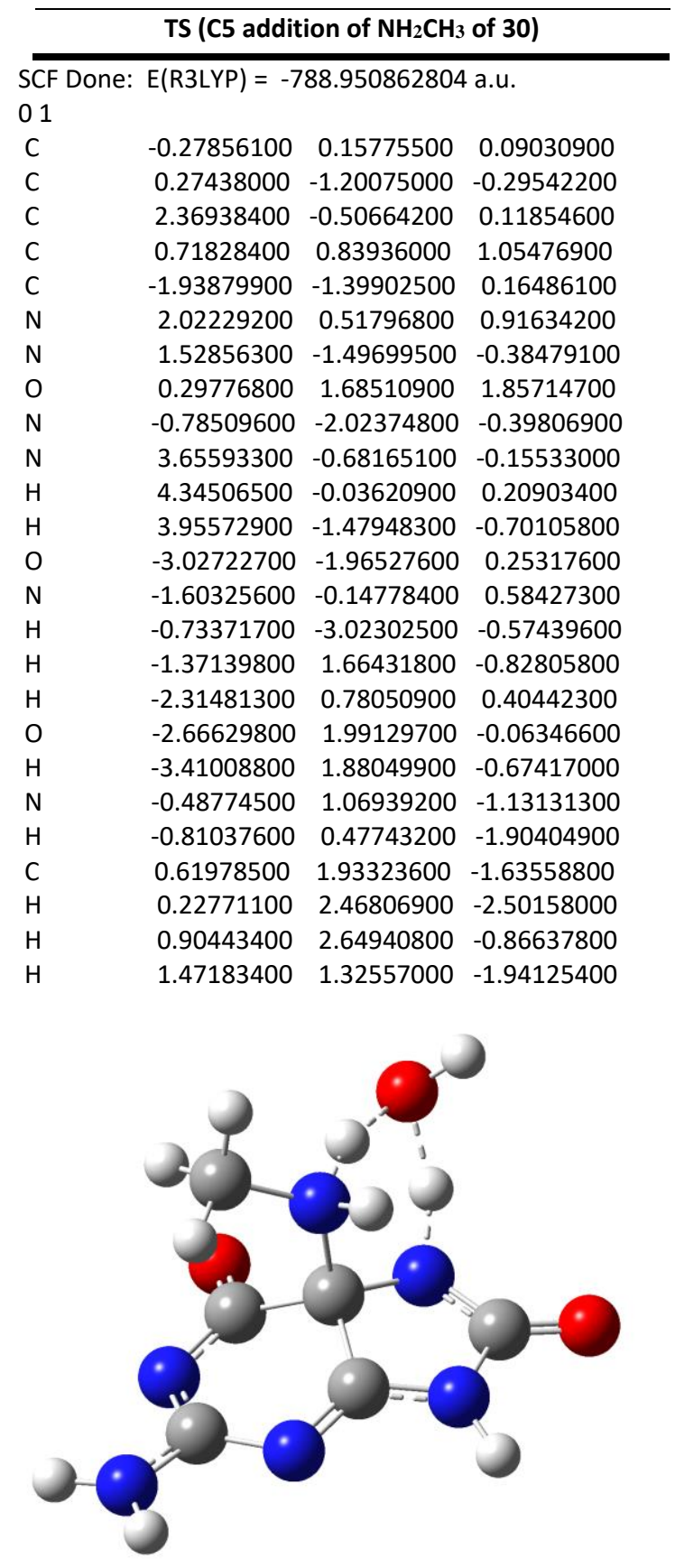




\section{TS (Acyl Group Migration (31AN $\rightarrow$ 32AN)}

$\begin{array}{lrrr}\text { SCF Done: } & E(R 3 L Y P)=-712.008207358 & \\ -11 & & & \\ \mathrm{C} & -0.46819000 & 0.48463900 & -0.48561000 \\ \mathrm{C} & 0.01767200 & -0.87774400 & -0.51756800 \\ \mathrm{C} & 2.18891500 & -0.60973800 & -0.04139300 \\ \mathrm{C} & 0.58696100 & 0.53954500 & 1.10289300 \\ \mathrm{C} & -2.18384200 & -0.98806000 & 0.02930100 \\ \mathrm{~N} & 1.88460900 & 0.26369600 & 0.96231500 \\ \mathrm{~N} & 1.27353300 & -1.29342300 & -0.74116000 \\ \mathrm{O} & 0.03887600 & 1.21855200 & 1.97996600 \\ \mathrm{~N} & -1.04514600 & -1.72356100 & -0.28992500 \\ \mathrm{~N} & 3.49883100 & -0.81574300 & -0.29778100 \\ \mathrm{H} & 4.17794900 & -0.46325000 & 0.36414500 \\ \mathrm{H} & 3.76266500 & -1.60217500 & -0.87759600 \\ \mathrm{~N} & -1.84965500 & 0.31747000 & -0.11096000 \\ \mathrm{H} & -0.94403700 & -2.69198000 & -0.00182000 \\ \mathrm{O} & -3.27414700 & -1.46927700 & 0.38463400 \\ \mathrm{~N} & -0.00405100 & 1.52922900 & -1.19919500 \\ \mathrm{C} & -0.59036800 & 2.81452900 & -0.83693100 \\ \mathrm{H} & -0.31481600 & 3.14509900 & 0.18093000 \\ \mathrm{H} & -0.22306900 & 3.57945000 & -1.52781700 \\ \mathrm{H} & -1.69233400 & 2.84173700 & -0.88831300 \\ \mathrm{H} & -2.49793300 & 1.07421200 & 0.06645400\end{array}$

TS (Acyl Group Migration (31AN' $\rightarrow$ 32AN')

$\begin{array}{lrrr}\text { SCF Done: } & E(R 3 L Y P)=-712.036772644 & \\ -11 & & & \\ \mathrm{C} & -0.57069800 & 0.50428800 & -0.49731500 \\ \mathrm{C} & -0.06454100 & -0.83231900 & -0.55745300 \\ \mathrm{C} & 2.11213600 & -0.71065900 & -0.05172100 \\ \mathrm{C} & 0.53506300 & 0.35943900 & 1.19481100 \\ \mathrm{C} & -2.25693600 & -0.81121900 & -0.05101100 \\ \mathrm{~N} & 1.81129800 & 0.05716200 & 1.05318700 \\ \mathrm{~N} & 1.19327400 & -1.25519200 & -0.84434200 \\ \mathrm{O} & -0.07074600 & 0.89675200 & 2.11594600 \\ \mathrm{~N} & -1.14346900 & -1.63641700 & -0.32285700 \\ \mathrm{~N} & 3.42107400 & -0.92958000 & -0.28835600 \\ \mathrm{H} & 4.08666300 & -0.69779900 & 0.43760400 \\ \mathrm{H} & 3.67648600 & -1.64187400 & -0.96073000 \\ \mathrm{~N} & -1.92506100 & 0.48404100 & -0.19626500 \\ \mathrm{H} & -1.08990400 & -2.61372000 & -0.05541100 \\ \mathrm{O} & -3.37717000 & -1.29521600 & 0.27757200 \\ \mathrm{~N} & -0.01084600 & 1.59293000 & -1.17295300 \\ \mathrm{C} & -0.20694800 & 2.93437000 & -0.60312300 \\ \mathrm{H} & 0.26716000 & 3.05025600 & 0.38060000 \\ \mathrm{H} & 0.22755600 & 3.66216300 & -1.29131200 \\ \mathrm{H} & -1.27271300 & 3.14765400 & -0.50610300 \\ \mathrm{H} & 0.97572900 & 1.42702800 & -1.35680700\end{array}$

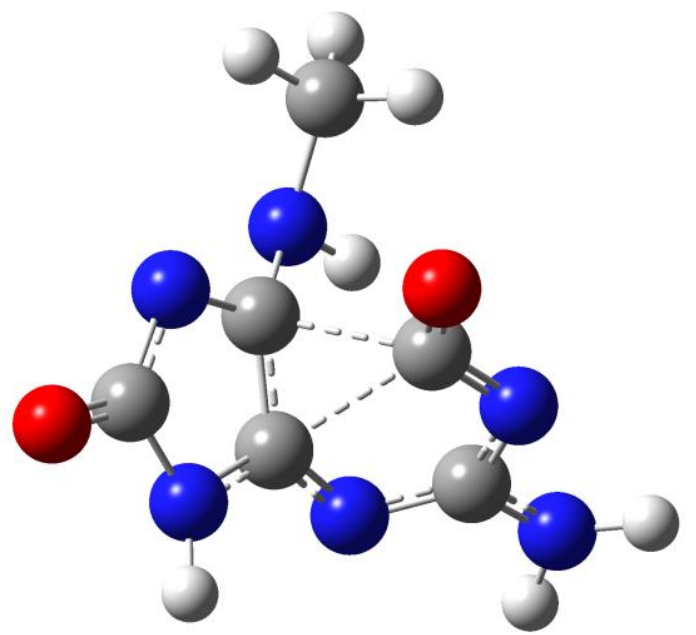



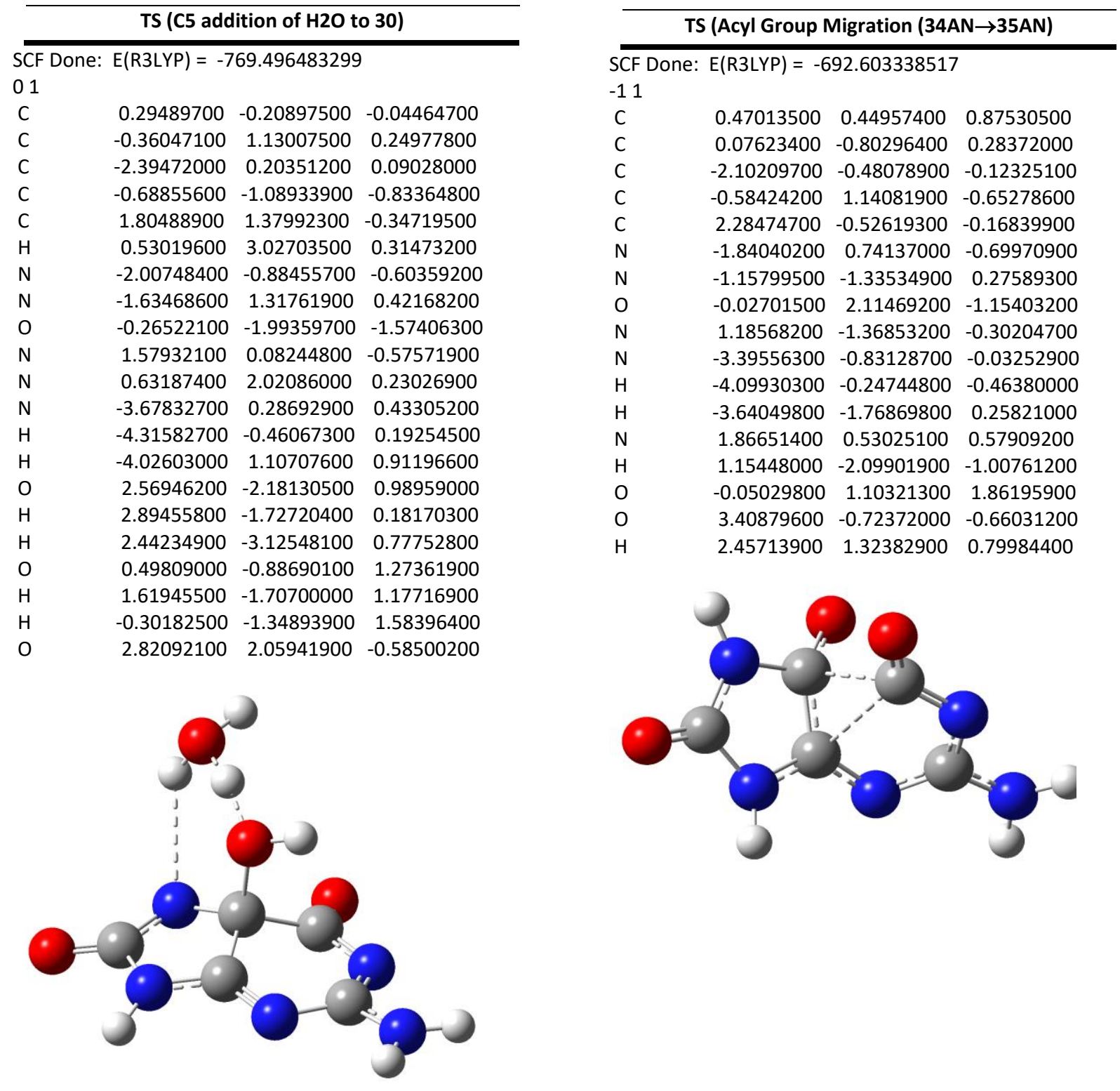

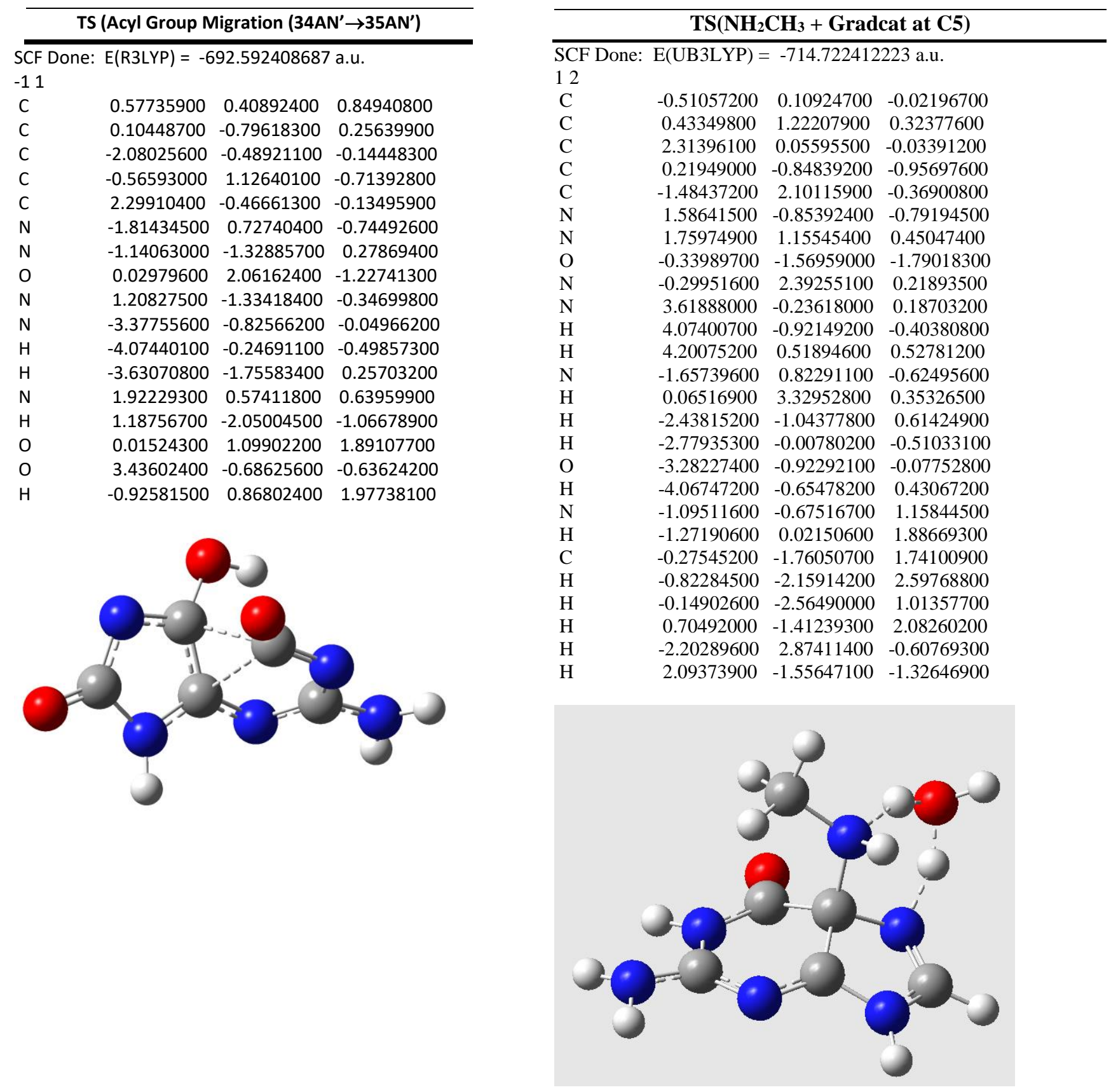


\begin{tabular}{lrrr}
\hline \multicolumn{4}{c}{ TS (Guanine radical $+\mathbf{N H}_{2} \mathbf{C H}_{3}$ at C5) } \\
\hline SCF Done: & E(UB3LYP) $=$ & -714.264111042 a.u. \\
$0 \mathrm{2}$ & & & \\
$\mathrm{C}$ & -0.27972700 & -1.86082500 & -1.67873500 \\
$\mathrm{H}$ & -0.84194200 & -2.27068600 & -2.51955900 \\
$\mathrm{H}$ & -0.18500500 & -2.62322700 & -0.90608600 \\
$\mathrm{~N}$ & -1.04855000 & -0.70615500 & -1.14680100 \\
$\mathrm{H}$ & -1.19280700 & -0.02617700 & -1.89980700 \\
$\mathrm{C}$ & -0.44792900 & 0.12223400 & 0.07893800 \\
$\mathrm{C}$ & 0.46871000 & 1.22635800 & -0.29990500 \\
$\mathrm{C}$ & 2.29121800 & -0.01046700 & 0.07742500 \\
$\mathrm{C}$ & 0.30519100 & -0.83784100 & 1.00846900 \\
$\mathrm{H}$ & 0.06490500 & 3.32508500 & -0.45534300 \\
$\mathrm{~N}$ & 1.63797400 & -0.90553500 & 0.86522300 \\
$\mathrm{~N}$ & 1.78681800 & 1.14297600 & -0.41775000 \\
$\mathrm{O}$ & -0.34508500 & -1.52343100 & 1.83937300 \\
$\mathrm{~N}$ & -0.28238800 & 2.39219000 & -0.25545000 \\
$\mathrm{~N}$ & 3.59370700 & -0.28369300 & -0.19830000 \\
$\mathrm{H}$ & 4.05447700 & -0.99770400 & 0.34900900 \\
$\mathrm{H}$ & 4.16827100 & 0.45495000 & -0.58109500 \\
$\mathrm{H}$ & 0.70320300 & -1.53520300 & -2.02146700 \\
$\mathrm{C}$ & -1.48533400 & 2.12786600 & 0.27307400 \\
$\mathrm{H}$ & -2.25033500 & 2.87503400 & 0.42605100 \\
$\mathrm{~N}$ & -1.60241400 & 0.83684500 & 0.60208300 \\
$\mathrm{H}$ & -2.04314100 & -0.98902600 & -0.77473600 \\
$\mathrm{O}$ & -3.35311700 & -0.94733400 & 0.08560600 \\
$\mathrm{H}$ & -2.52989100 & 0.25483400 & 0.57260500 \\
$\mathrm{H}$ & -4.07091900 & -0.64210600 & -0.48804600
\end{tabular}

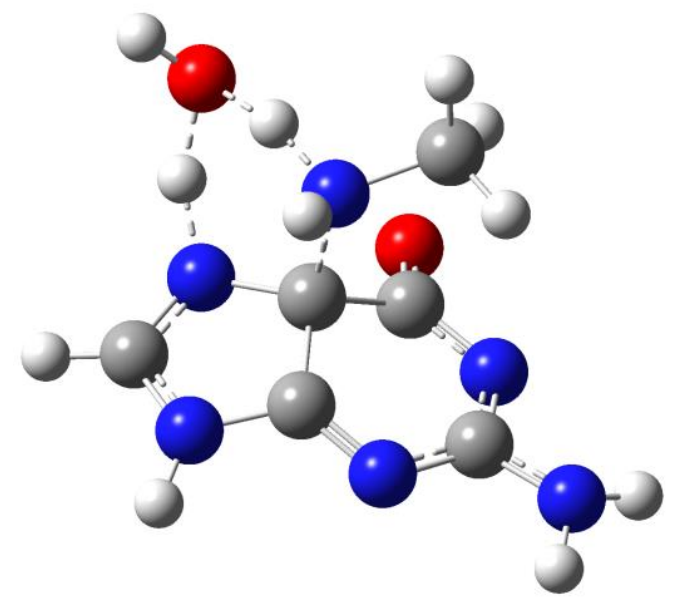




\begin{tabular}{|c|c|c|c|}
\hline \multicolumn{4}{|c|}{ TS (Acyl Group Migration (46 $\rightarrow 47$ ) } \\
\hline \multicolumn{4}{|c|}{$\begin{array}{l}\text { SCF Done: } E(\text { R3LYP) }=-731.919831368 \text { a.u. } \\
01\end{array}$} \\
\hline $\mathrm{C}$ & -0.11836600 & 0.54210000 & -0.53648300 \\
\hline C & 0.28924300 & -0.82341500 & -0.59700100 \\
\hline C & 2.44140600 & -0.83226500 & 0.01265000 \\
\hline C & 0.92215000 & 0.39371800 & 1.21385300 \\
\hline C & -1.88235600 & -0.64726600 & -0.16331100 \\
\hline $\mathrm{N}$ & 2.16162600 & -0.01728200 & 1.08644200 \\
\hline $\mathrm{N}$ & 1.51853000 & -1.33574500 & -0.80346100 \\
\hline $\mathrm{O}$ & 0.32299900 & 0.99106400 & 2.09046000 \\
\hline $\mathrm{N}$ & -0.85346500 & -1.55432700 & -0.38259300 \\
\hline $\mathrm{N}$ & 3.73181700 & -1.16479800 & -0.15467200 \\
\hline $\mathrm{H}$ & 4.41690800 & -0.87022600 & 0.52605200 \\
\hline $\mathrm{H}$ & 3.98809700 & -1.84553300 & -0.85620600 \\
\hline $\mathrm{N}$ & -1.49346900 & 0.60803400 & -0.28998800 \\
\hline $\mathrm{H}$ & -0.87282000 & -2.53928700 & -0.13909300 \\
\hline $\mathrm{N}$ & -3.11285300 & -1.07445600 & 0.18558400 \\
\hline $\mathrm{H}$ & -3.31683700 & -2.04927800 & 0.00227700 \\
\hline C & -4.24653700 & -0.15489700 & 0.22430000 \\
\hline $\mathrm{H}$ & -4.46813800 & 0.26696500 & -0.76272300 \\
\hline $\mathrm{H}$ & -5.11776300 & -0.70653800 & 0.57777900 \\
\hline $\mathrm{H}$ & -4.04528600 & 0.66306300 & 0.91910600 \\
\hline $\mathrm{N}$ & 0.51543900 & 1.58978100 & -1.21232500 \\
\hline $\mathrm{H}$ & 1.48089000 & 1.35788600 & -1.42729200 \\
\hline C & 0.41472500 & 2.94380200 & -0.65287200 \\
\hline $\mathrm{H}$ & 0.83469700 & 3.64512400 & -1.37657600 \\
\hline $\mathrm{H}$ & -0.63267100 & 3.20297300 & -0.49412900 \\
\hline $\mathrm{H}$ & 0.95396200 & 3.05723700 & 0.29740000 \\
\hline
\end{tabular}

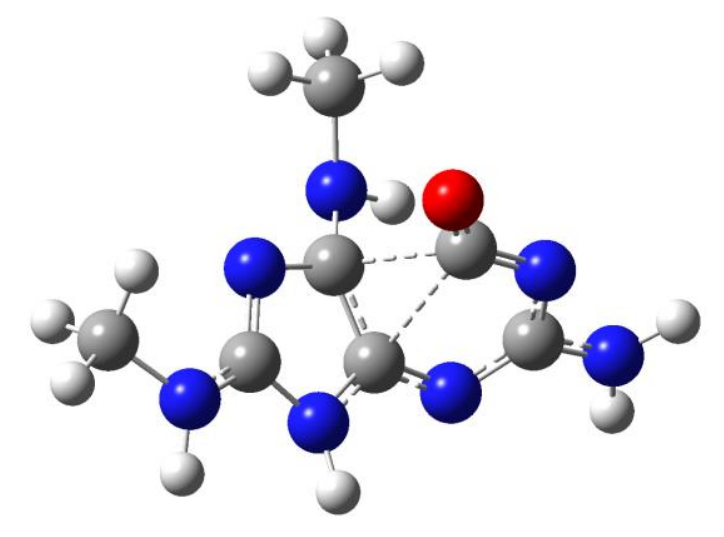

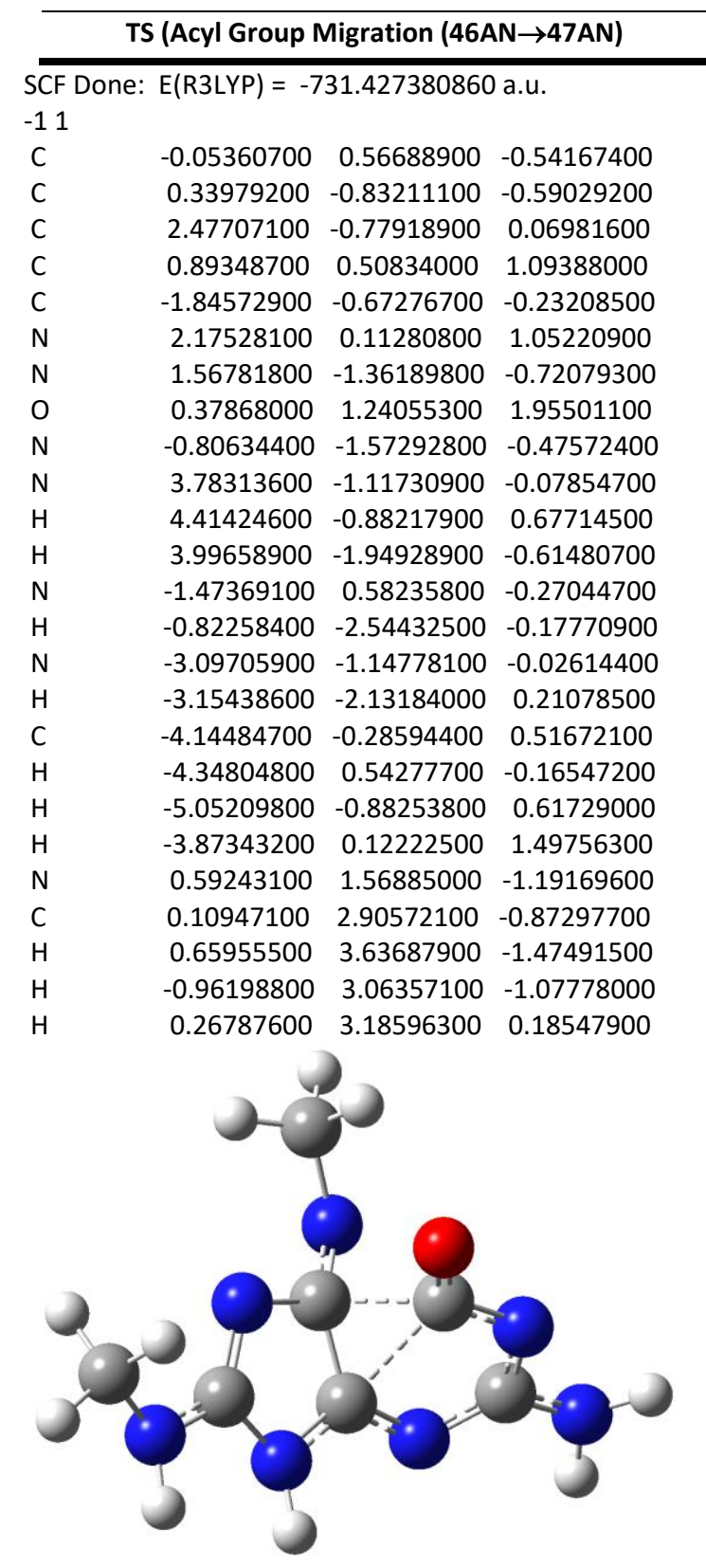




\begin{tabular}{|c|c|c|c|}
\hline \multicolumn{4}{|c|}{ TS (Acyl Group Migration (46'ANa $\rightarrow 47^{\prime} \mathrm{ANa}$ ) } \\
\hline \multicolumn{4}{|c|}{ SCF Done: $E(R 3 L Y P)=-731.447910738$ a.u. } \\
\hline \multicolumn{4}{|c|}{-11} \\
\hline C & -0.18204300 & 0.53864800 & -0.54608700 \\
\hline C & 0.28536700 & -0.81511900 & -0.59898700 \\
\hline C & 2.44079500 & -0.77399400 & 0.02291200 \\
\hline C & 0.84669900 & 0.35215200 & 1.19030900 \\
\hline C & -1.93435300 & -0.73781700 & -0.20813800 \\
\hline $\mathrm{N}$ & 2.12185700 & -0.00001800 & 1.11677600 \\
\hline $\mathrm{N}$ & 1.54323700 & -1.27771700 & -0.81999200 \\
\hline 0 & 0.23964300 & 0.92506200 & 2.09642600 \\
\hline $\mathrm{N}$ & -0.82259200 & -1.58936600 & -0.44925300 \\
\hline $\mathrm{N}$ & 3.75065200 & -1.04992700 & -0.15495200 \\
\hline $\mathrm{H}$ & 4.38998000 & -0.85134300 & 0.60358500 \\
\hline $\mathrm{H}$ & 4.00310900 & -1.77795700 & -0.81143000 \\
\hline $\mathrm{N}$ & -1.54509200 & 0.56425900 & -0.33103600 \\
\hline $\mathrm{H}$ & -0.79189600 & -2.55875000 & -0.15158100 \\
\hline $\mathrm{N}$ & -3.09751200 & -1.25612900 & 0.07932000 \\
\hline C & -4.15849000 & -0.28497500 & 0.33147400 \\
\hline $\mathrm{H}$ & -4.41865400 & 0.30392000 & -0.56079300 \\
\hline $\mathrm{H}$ & -5.06286300 & -0.81371200 & 0.64506200 \\
\hline $\mathrm{H}$ & -3.90233700 & 0.43164700 & 1.12553700 \\
\hline$N$ & 0.45683600 & 1.61470700 & -1.17643800 \\
\hline C & 0.21738500 & 2.96094000 & -0.63726500 \\
\hline $\mathrm{H}$ & 0.72843400 & 3.68118400 & -1.27933500 \\
\hline $\mathrm{H}$ & -0.85011300 & 3.18634900 & -0.65357700 \\
\hline $\mathrm{H}$ & 0.58753700 & 3.07971100 & 0.38992700 \\
\hline $\mathrm{H}$ & 1.45579300 & 1.43876800 & -1.25508500 \\
\hline
\end{tabular}

\begin{tabular}{lrrr}
\hline \multicolumn{3}{c}{ TS (Acyl Group Migration (46'ANb $\rightarrow$ 47'ANb) } \\
SCF Done: & E(R3LYP) $=-731.426307173$ & a.u. \\
-11 & & & \\
$\mathrm{C}$ & -0.05687000 & 0.58067700 & -0.57806000 \\
$\mathrm{C}$ & 0.36966100 & -0.79885900 & -0.66095300 \\
$\mathrm{C}$ & 2.48152600 & -0.70655800 & 0.07576200 \\
$\mathrm{C}$ & 0.81073300 & 0.43387500 & 1.11493100 \\
$\mathrm{C}$ & -1.85931900 & -0.84790100 & -0.23989400 \\
$\mathrm{~N}$ & 2.10327600 & 0.09139600 & 1.11788700 \\
$\mathrm{~N}$ & 1.62818500 & -1.25743000 & -0.79506200 \\
$\mathrm{O}$ & 0.19476200 & 1.06776700 & 1.98606100 \\
$\mathrm{~N}$ & -0.73361600 & -1.61123600 & -0.59215000 \\
$\mathrm{~N}$ & 3.80374900 & -0.97453500 & -0.04302400 \\
$\mathrm{H}$ & 4.40094700 & -0.75356200 & 0.74350900 \\
$\mathrm{H}$ & 4.08201400 & -1.74647700 & -0.63609300 \\
$\mathrm{~N}$ & -1.48080400 & 0.46998800 & -0.37432400 \\
$\mathrm{H}$ & -0.66130200 & -2.57770800 & -0.28844600 \\
$\mathrm{~N}$ & -2.97672400 & -1.37252300 & 0.12383600 \\
$\mathrm{C}$ & -4.03445400 & -0.42402200 & 0.47788300 \\
$\mathrm{H}$ & -4.36970200 & 0.16056300 & -0.38953100 \\
$\mathrm{H}$ & -4.89568700 & -0.97562700 & 0.85978100 \\
$\mathrm{H}$ & -3.71762700 & 0.28558400 & 1.25480300 \\
$\mathrm{~N}$ & 0.53660800 & 1.64595200 & -1.15223000 \\
$\mathrm{C}$ & -0.04089500 & 2.93034500 & -0.77092400 \\
$\mathrm{H}$ & 0.42871400 & 3.72287900 & -1.36170100 \\
$\mathrm{H}$ & -1.12762900 & 3.00646600 & -0.94439700 \\
$\mathrm{H}$ & 0.12835700 & 3.18417900 & 0.29129800 \\
$\mathrm{H}$ & -2.01320000 & 1.20493600 & 0.07529000 \\
& & & \\
\hline & & & \\
& & &
\end{tabular}
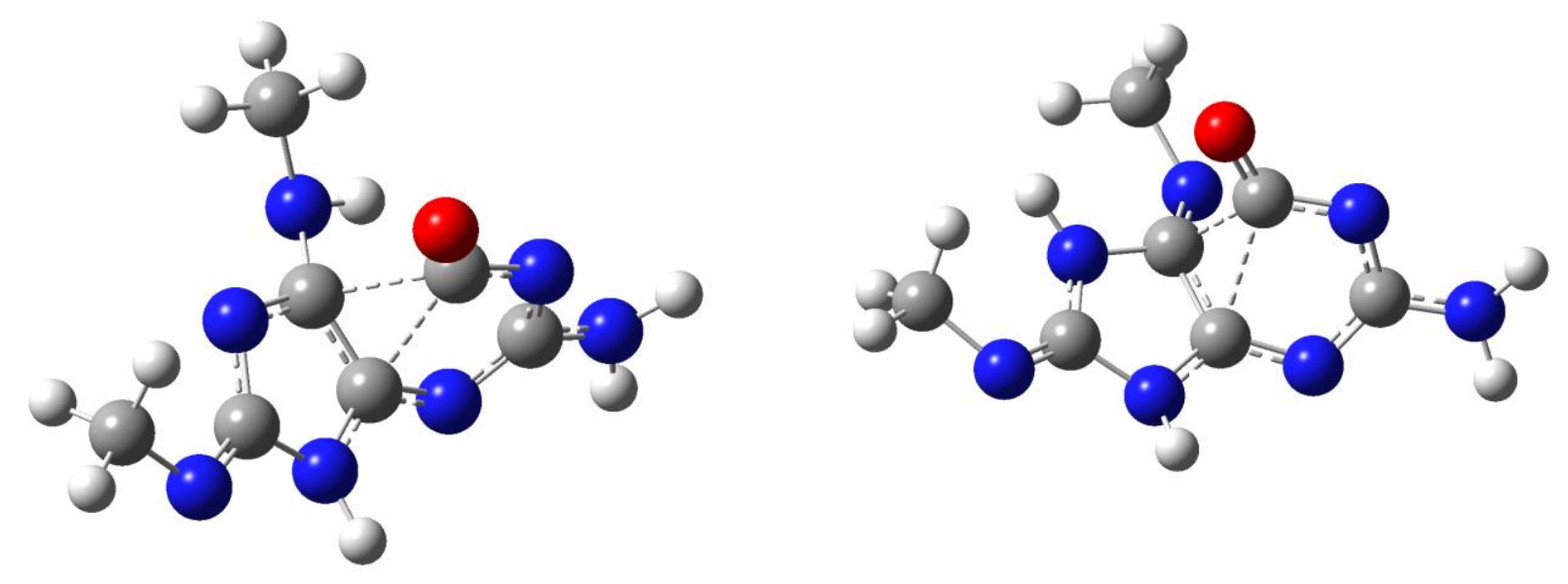


\section{TS (C8 addition of $\mathrm{H}_{2} \mathrm{O}$ to 42)}

\begin{tabular}{lrrr}
\hline SCF Done: & E(R3LYP) $=-790.121783382$ & a.u. \\
O 1 & & & \\
C & -0.15257600 & 0.46169900 & 0.40055000 \\
C & -0.54419900 & -0.95949400 & 0.75102900 \\
C & -2.40475800 & -0.72330900 & -0.46493900 \\
$\mathrm{C}$ & -0.67374600 & 0.70400800 & -1.03755700 \\
$\mathrm{C}$ & 1.64408700 & -0.78030400 & 1.09886900 \\
$\mathrm{~N}$ & -1.90065500 & 0.19360500 & -1.29840700 \\
$\mathrm{~N}$ & -1.66416100 & -1.50036700 & 0.44411200 \\
$\mathrm{O}$ & -0.01828700 & 1.38961400 & -1.82963200 \\
$\mathrm{~N}$ & 0.54652400 & -1.55603800 & 1.32716400 \\
$\mathrm{~N}$ & -3.68334000 & -1.05683900 & -0.55673500 \\
$\mathrm{H}$ & -4.28724000 & -0.58219200 & -1.21664600 \\
$\mathrm{H}$ & -4.05549900 & -1.80513500 & 0.01440200 \\
$\mathrm{~N}$ & 1.31221900 & 0.37299100 & 0.57124000 \\
$\mathrm{H}$ & 0.59476300 & -2.52497200 & 1.63026900 \\
$\mathrm{C}$ & -0.51839400 & 2.82363100 & 1.03217800 \\
$\mathrm{H}$ & -0.94920100 & 3.40441500 & 1.84984700 \\
$\mathrm{H}$ & -1.00099100 & 3.13873100 & 0.10461400 \\
$\mathrm{~N}$ & -0.80769000 & 1.40200100 & 1.29842200 \\
$\mathrm{H}$ & -0.52734200 & 1.17211800 & 2.25125300 \\
$\mathrm{H}$ & 3.48150500 & -0.37415400 & -0.85665900 \\
$\mathrm{H}$ & 0.55323500 & 3.05258100 & 0.97293600 \\
$\mathrm{H}$ & 2.62366700 & -1.04255600 & 1.45940100 \\
$\mathrm{O}$ & 3.72330500 & 0.63717300 & -0.89406700 \\
$\mathrm{H}$ & 4.64785900 & 0.70640500 & -0.61928200 \\
$\mathrm{H}$ & 2.02191300 & 1.01031700 & 0.20109000 \\
$\mathrm{O}$ & 3.01574100 & -1.81537200 & -0.71659500 \\
$\mathrm{H}$ & 2.40849500 & -1.99173300 & -1.45022900
\end{tabular}

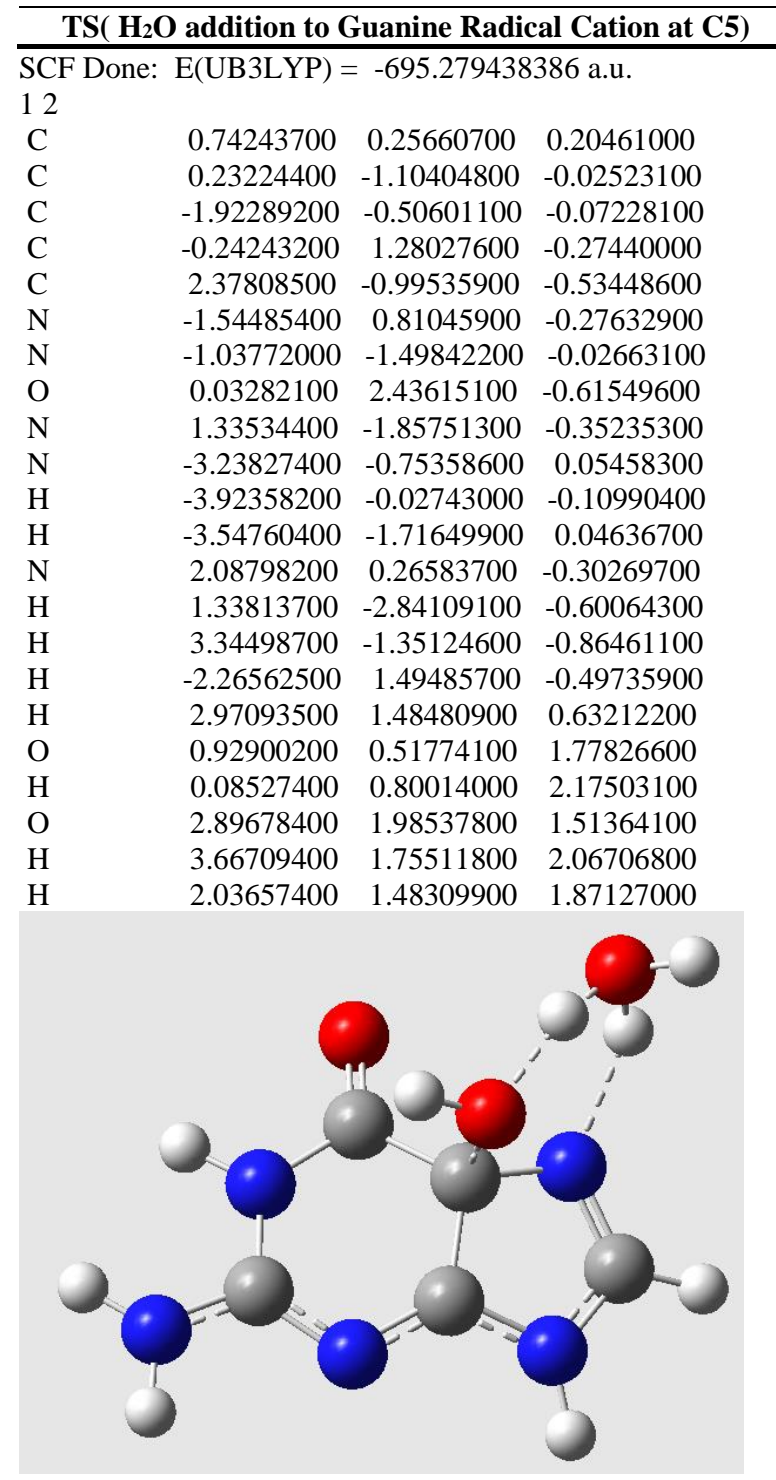




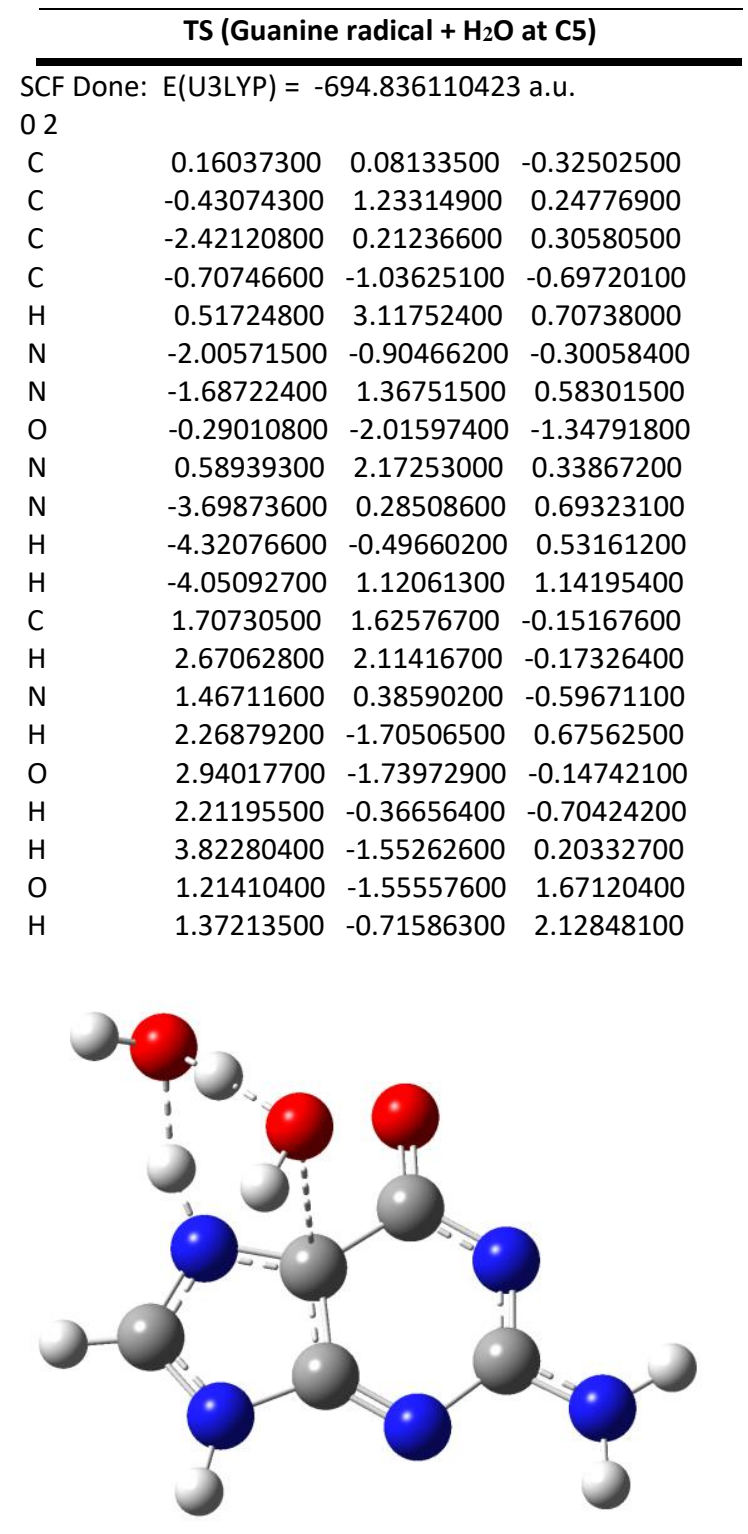




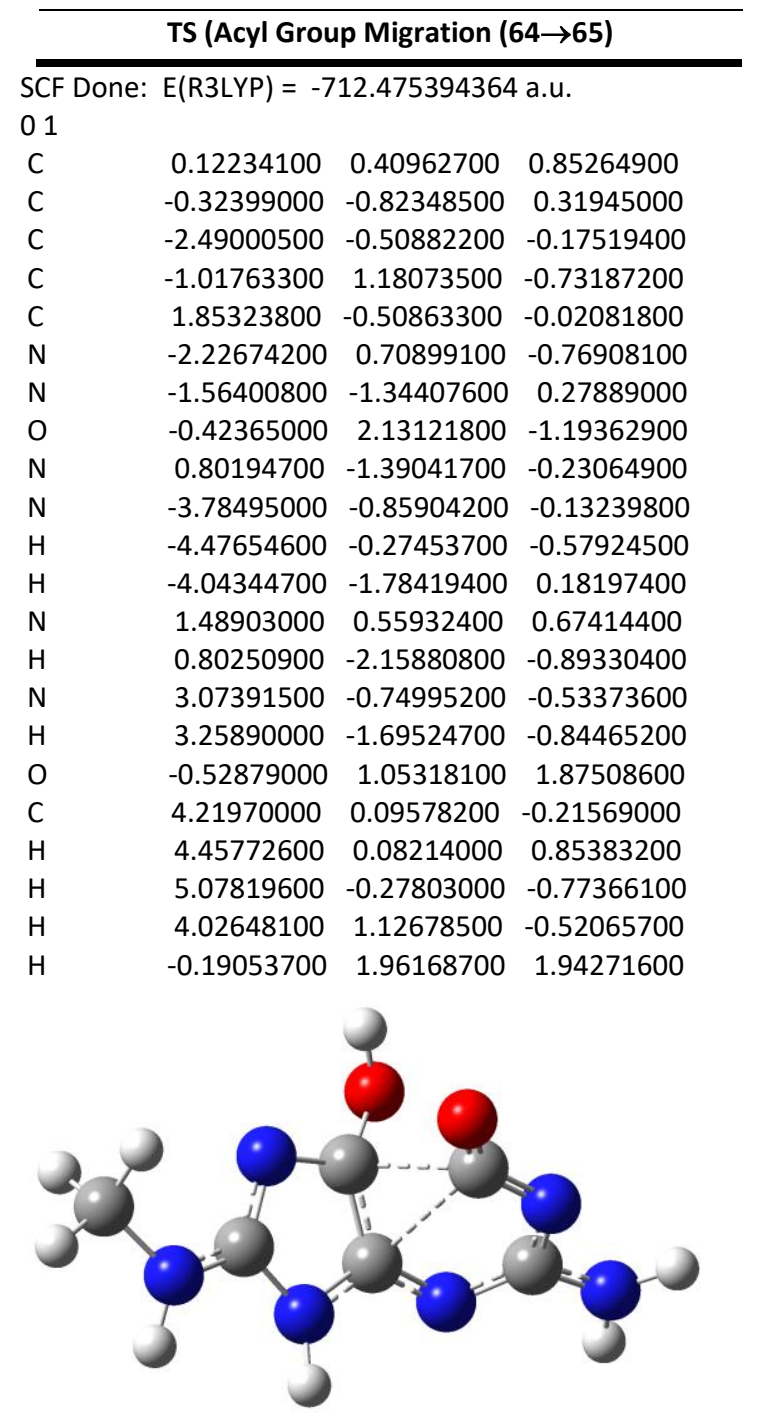




\section{TS (Acyl Group Migration (64AN $\rightarrow$ 65AN)}

$\begin{array}{lrrr}\text { SCF Done: } & E(R 3 L Y P)=-712.026078694 & \text { a.u. } \\ -11 & & & \\ \text { C } & 0.06682400 & 0.44886000 & 0.89457900 \\ \text { C } & -0.33226600 & -0.80660500 & 0.29203000 \\ \text { C } & -2.49430600 & -0.47457800 & -0.16197900 \\ \text { C } & -0.94586500 & 1.14009000 & -0.62422000 \\ \text { C } & 1.83349300 & -0.45310900 & -0.03300500 \\ \text { N } & -2.21241800 & 0.75535200 & -0.70542900 \\ \text { N } & -1.56646000 & -1.34227600 & 0.24354300 \\ \text { O } & -0.40027300 & 2.12786300 & -1.12224700 \\ \text { N } & 0.79284600 & -1.35192100 & -0.25898900 \\ \text { N } & -3.79686400 & -0.82021400 & -0.10139700 \\ \text { H } & -4.47082700 & -0.25176100 & -0.59713600 \\ \text { H } & -4.03676600 & -1.78316900 & 0.09648600 \\ \text { N } & 1.47041900 & 0.59804400 & 0.66959000 \\ H & 0.80354000 & -2.06922600 & -0.97899200 \\ \text { N } & 3.05095600 & -0.70047900 & -0.56604400 \\ \text { H } & 3.21234200 & -1.66675700 & -0.82876400 \\ \text { O } & -0.52487400 & 1.07341900 & 1.87838700 \\ \mathrm{C} & 4.22573100 & 0.05437200 & -0.13430500 \\ \mathrm{H} & 4.42479000 & -0.07463800 & 0.93617300 \\ \mathrm{H} & 5.08438400 & -0.30638400 & -0.70142700 \\ \mathrm{H} & 4.09268800 & 1.11795300 & -0.34298000\end{array}$

\section{TS (Acyl Group Migration (64aAN $\rightarrow 65 a A N)$}

\begin{tabular}{|c|c|c|c|}
\hline \multicolumn{4}{|c|}{$\begin{array}{l}\text { SCF Done: } E(R 3 L Y P)=-712.021778388 \text { a.u. } \\
-11\end{array}$} \\
\hline C & 0.07502100 & 0.38294900 & 0.96656700 \\
\hline C & -0.35087300 & -0.82579100 & 0.31422500 \\
\hline C & -2.48484700 & -0.39986700 & -0.20641900 \\
\hline C & -0.84469300 & 1.11542600 & -0.63835600 \\
\hline C & 1.88232100 & -0.63302900 & -0.05667700 \\
\hline $\mathrm{N}$ & -2.12374500 & 0.80581100 & -0.76654000 \\
\hline $\mathrm{N}$ & -1.61383200 & -1.30001700 & 0.25010600 \\
\hline $\mathrm{O}$ & -0.19776400 & 2.05305300 & -1.10960100 \\
\hline $\mathrm{N}$ & 0.75012400 & -1.44579300 & -0.22076200 \\
\hline$N$ & -3.80188600 & -0.67639800 & -0.18914100 \\
\hline $\mathrm{H}$ & -4.43922200 & -0.06204000 & -0.67783900 \\
\hline $\mathrm{H}$ & -4.11391300 & -1.60545200 & 0.06094000 \\
\hline $\mathrm{N}$ & 1.48767500 & 0.39849300 & 0.77491800 \\
\hline $\mathrm{H}$ & 0.68968700 & -2.09245300 & -1.00177500 \\
\hline$N$ & 3.01773000 & -0.87996300 & -0.60730900 \\
\hline $\mathrm{O}$ & -0.48599100 & 1.05500300 & 1.91625100 \\
\hline C & 4.08304200 & 0.08097400 & -0.31781000 \\
\hline $\mathrm{H}$ & 4.32688700 & 0.11423900 & 0.75265100 \\
\hline $\mathrm{H}$ & 4.98571600 & -0.21335300 & -0.85660000 \\
\hline $\mathrm{H}$ & 3.82152100 & 1.10118600 & -0.63140700 \\
\hline $\mathrm{H}$ & 2.02708200 & 1.25451900 & 0.84275900 \\
\hline
\end{tabular}

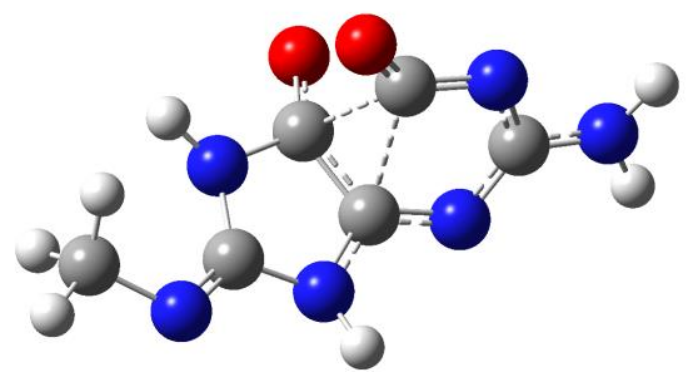




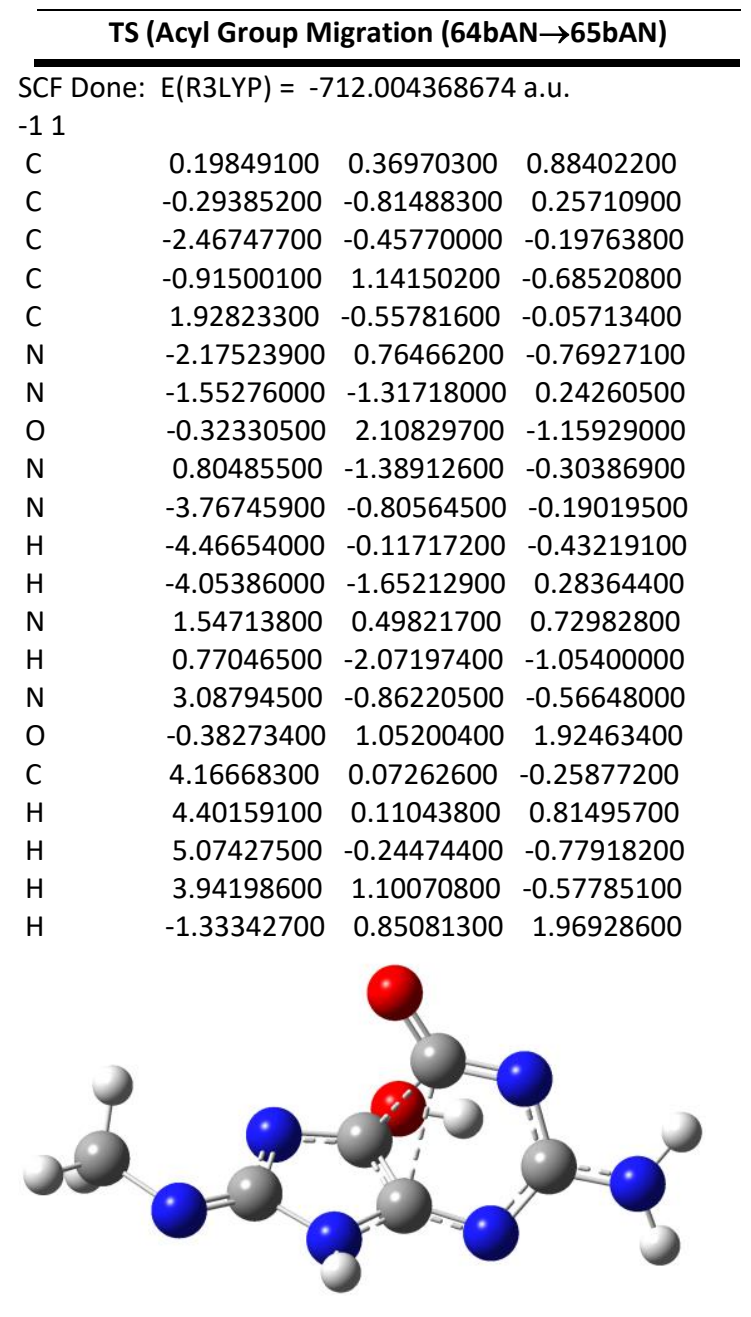




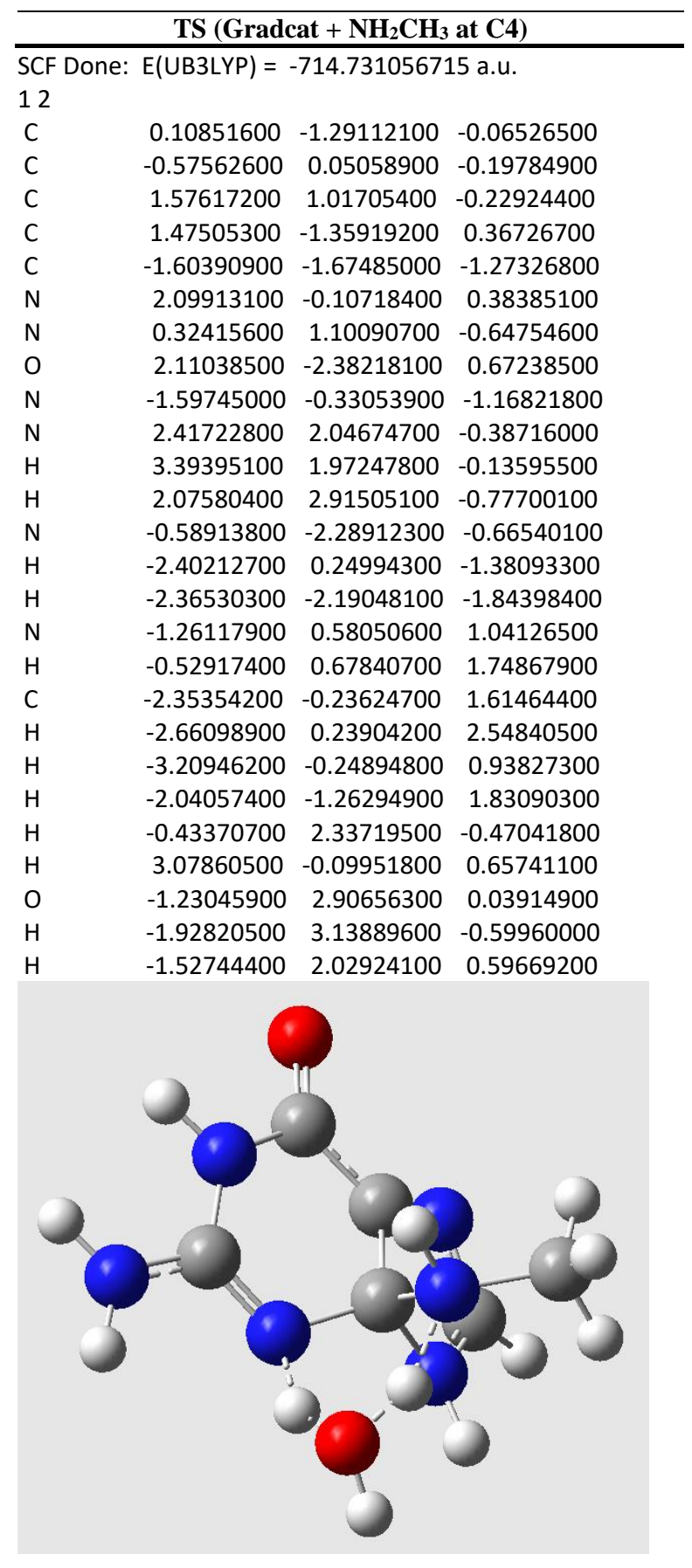




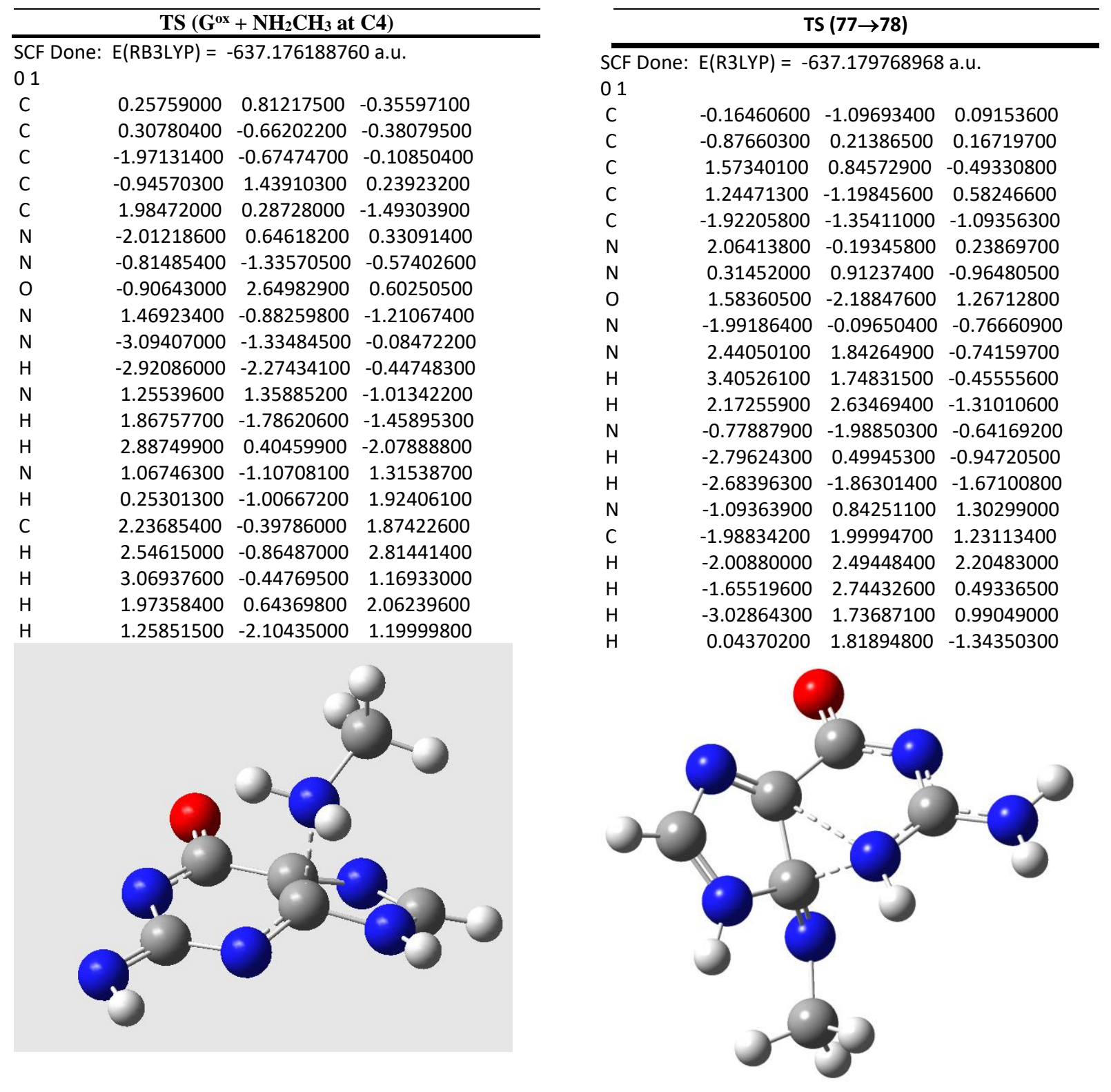



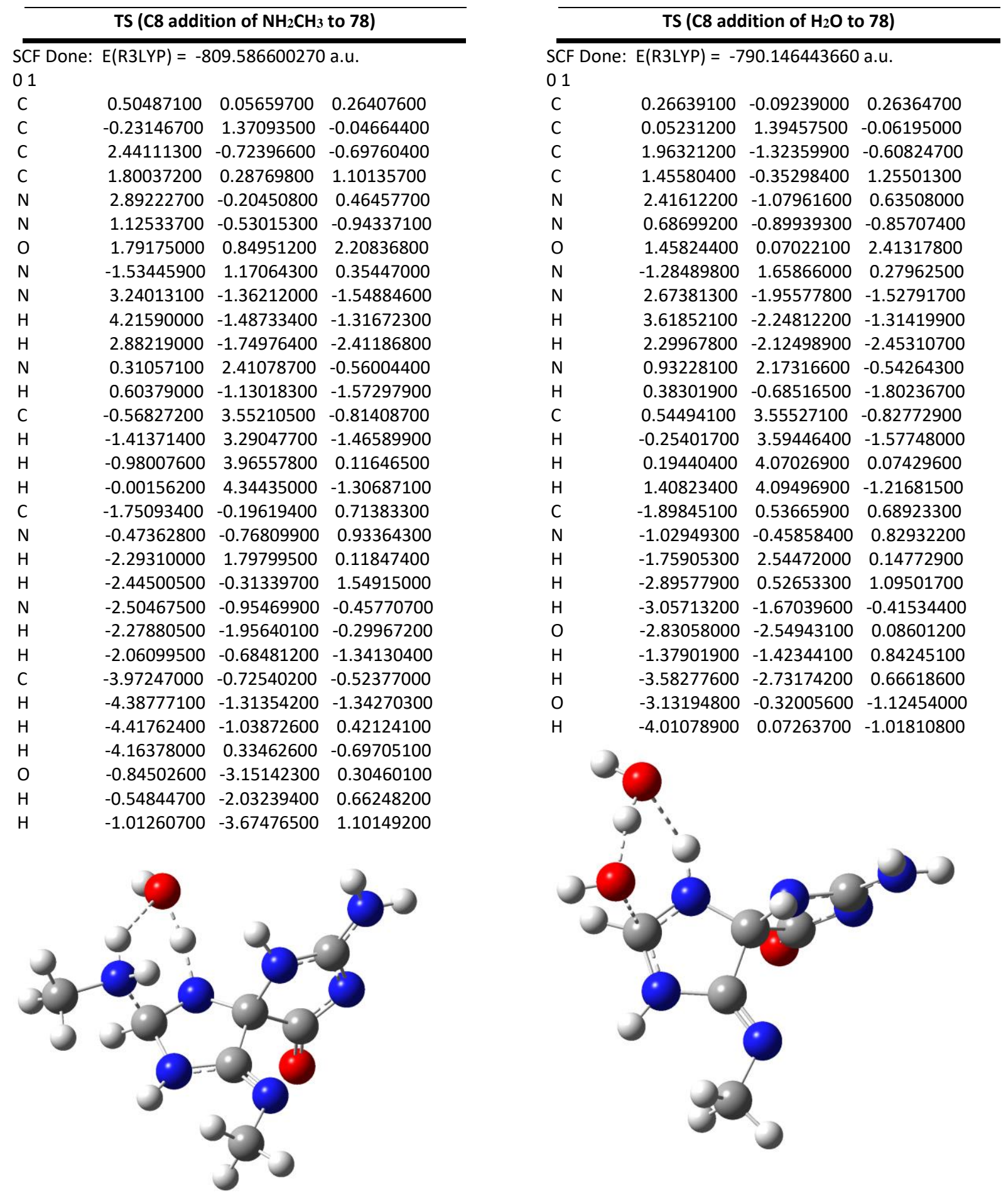
TS(Guanine Radical Cation at $\mathrm{C4}+\mathrm{H}_{2} \mathrm{O}$ at $\mathrm{C4}$ )

SCF Done: $\mathrm{E}(\mathrm{UB} 3 \mathrm{LYP})=-695.275237041$ a.u.

12

C

C

C

$\mathrm{C}$

C

$\mathrm{N}$

$\mathrm{N}$

$\mathrm{O}$

$\mathrm{N}$

$\mathrm{N}$

$\mathrm{H}$

$\mathrm{H}$

$\mathrm{N}$

$\mathrm{H}$

$\mathrm{H}$

$\mathrm{H}$

$\mathrm{H}$

$\mathrm{O}$

$\begin{array}{llll}\mathrm{H} & -0.44442900 & -2.26592900 & 0.20847800\end{array}$

O $\quad-0.81660400 \quad-2.71788100 \quad-0.64221200$

$\mathrm{H} \quad-0.14427300 \quad-3.33780500 \quad-0.97082900$

$\mathrm{H} \quad 0.85934500 \quad-1.22662200 \quad 1.53191700$

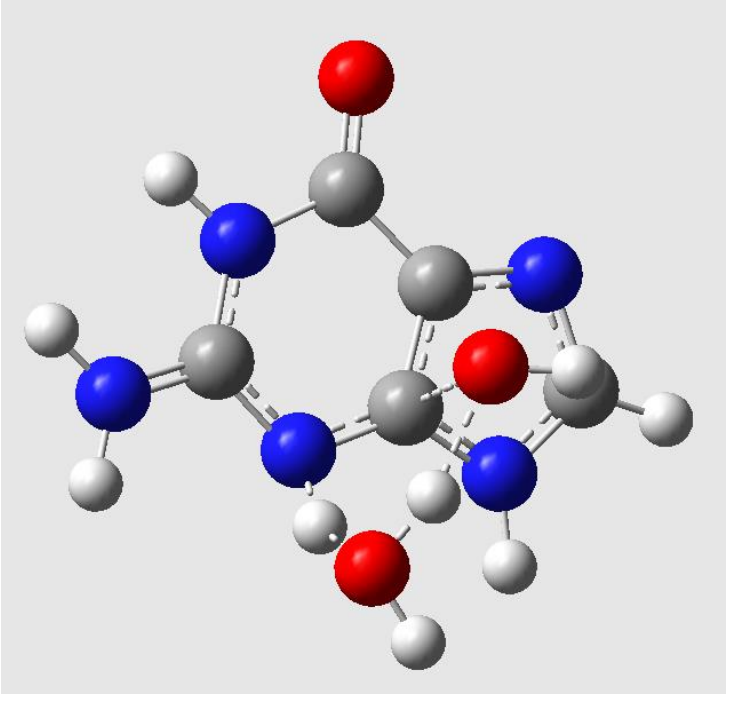

TS (Guanine radical $+\mathrm{H}_{2} \mathrm{O}$ at $\mathrm{C} 4$ )

SCF Done: $E(U 3 L Y P)=-694.830658427$ a.u.

02

$\begin{array}{llll}\text { C } & -0.01663800 & 1.25303800 & -0.30880800\end{array}$

$\begin{array}{llll}\text { C } & -0.26669600 & -0.08479800 & -0.73637900\end{array}$

C $\quad 1.93805900 \quad-0.63114900-0.31620100$

$\begin{array}{llll}\text { C } & 1.34394200 & 1.60415700 & 0.11454800\end{array}$

H $\quad-2.09016700 \quad-0.90327300 \quad-1.48546100$

N $\quad 2.26650700 \quad 0.59169000 \quad 0.07203700$

N $\quad 0.68790600 \quad-1.01021200 \quad-0.82498200$

$\begin{array}{lllll}\mathrm{O} & 1.64231200 & 2.75577700 & 0.48135300\end{array}$

N $\quad-1.56031300 \quad-0.10136700 \quad-1.15457000$

N $\quad 2.83459500 \quad-1.61467700 \quad-0.25171800$

H $\quad 3.75164000-1.41856800 \quad 0.12903300$

H $\quad 2.60913400-2.56148800-0.53281400$

$\begin{array}{lllll}\text { C } & -2.04679300 & 1.15413900 & -0.93433200\end{array}$

H $\quad-3.07454800 \quad 1.40760000 \quad-1.15199800$

N $\quad-1.13228500 \quad 1.99718400 \quad-0.43502400$

H $\quad-0.75308100 \quad-2.42414500 \quad 0.81235000$

$\begin{array}{lllll}0 & -0.47997000 & -3.17000300 & 0.10951900\end{array}$

H $\quad 0.38113700 \quad-2.02548700 \quad-0.72770000$

H $\quad 0.13229600 \quad-3.77042300 \quad 0.55933300$

$\begin{array}{lllll}0 & -1.01756400 & -1.24609500 & 1.63032800\end{array}$

H $\quad-1.94972500 \quad-0.99664200 \quad 1.54674900$

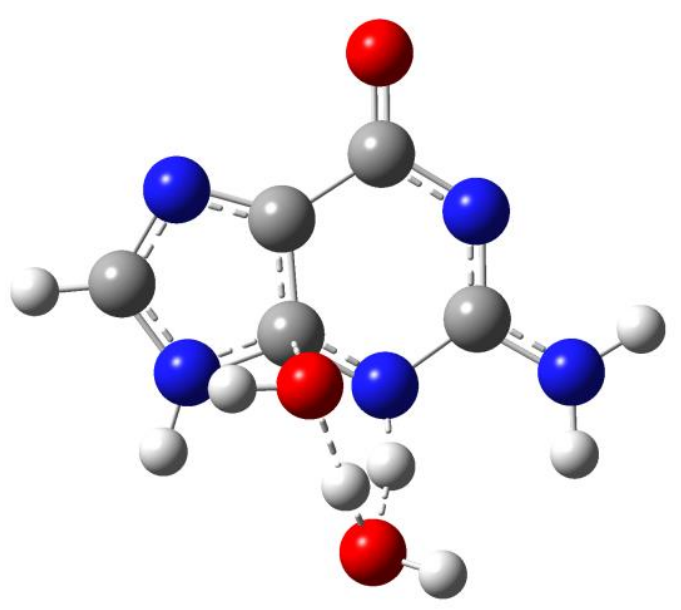



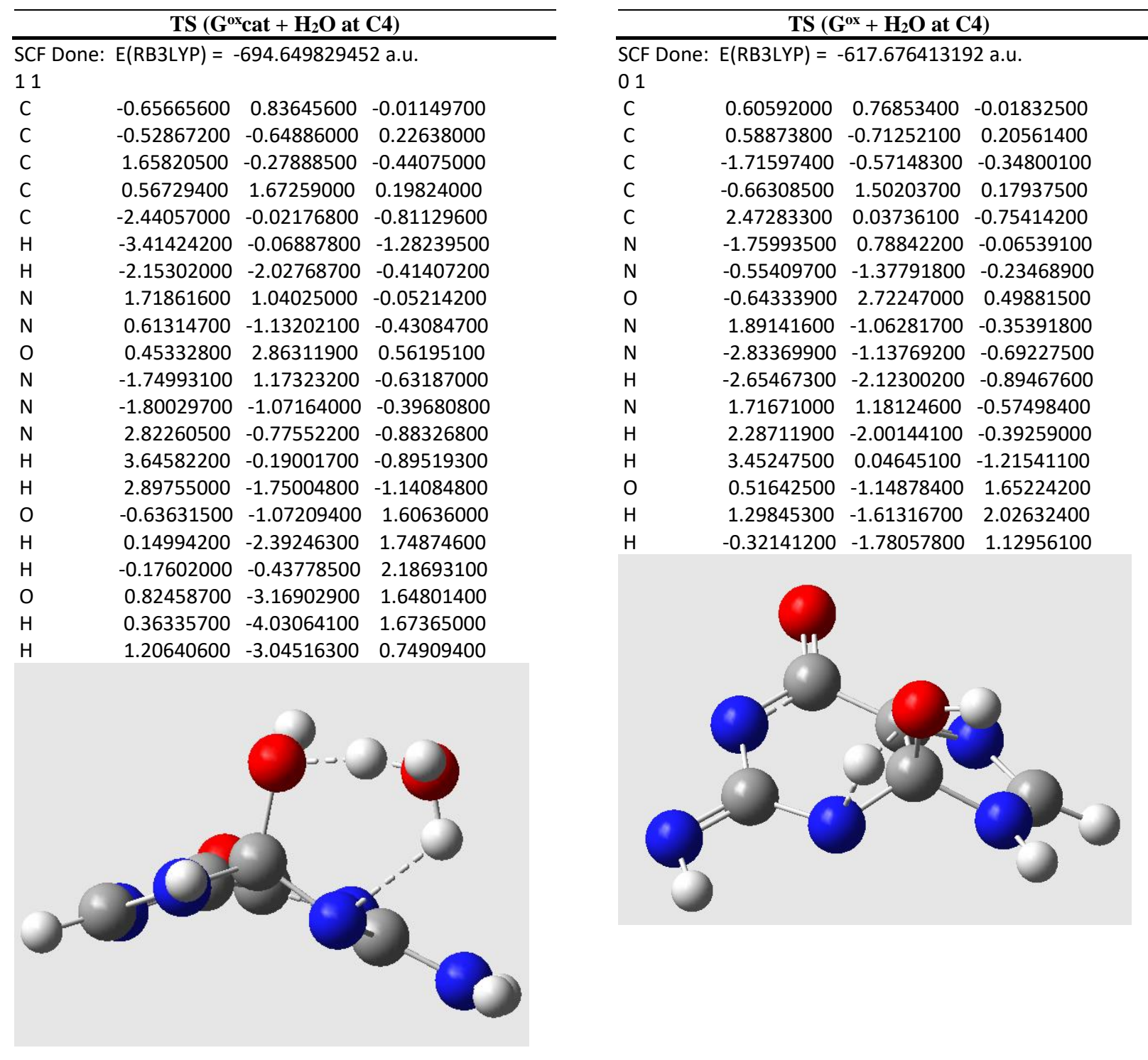

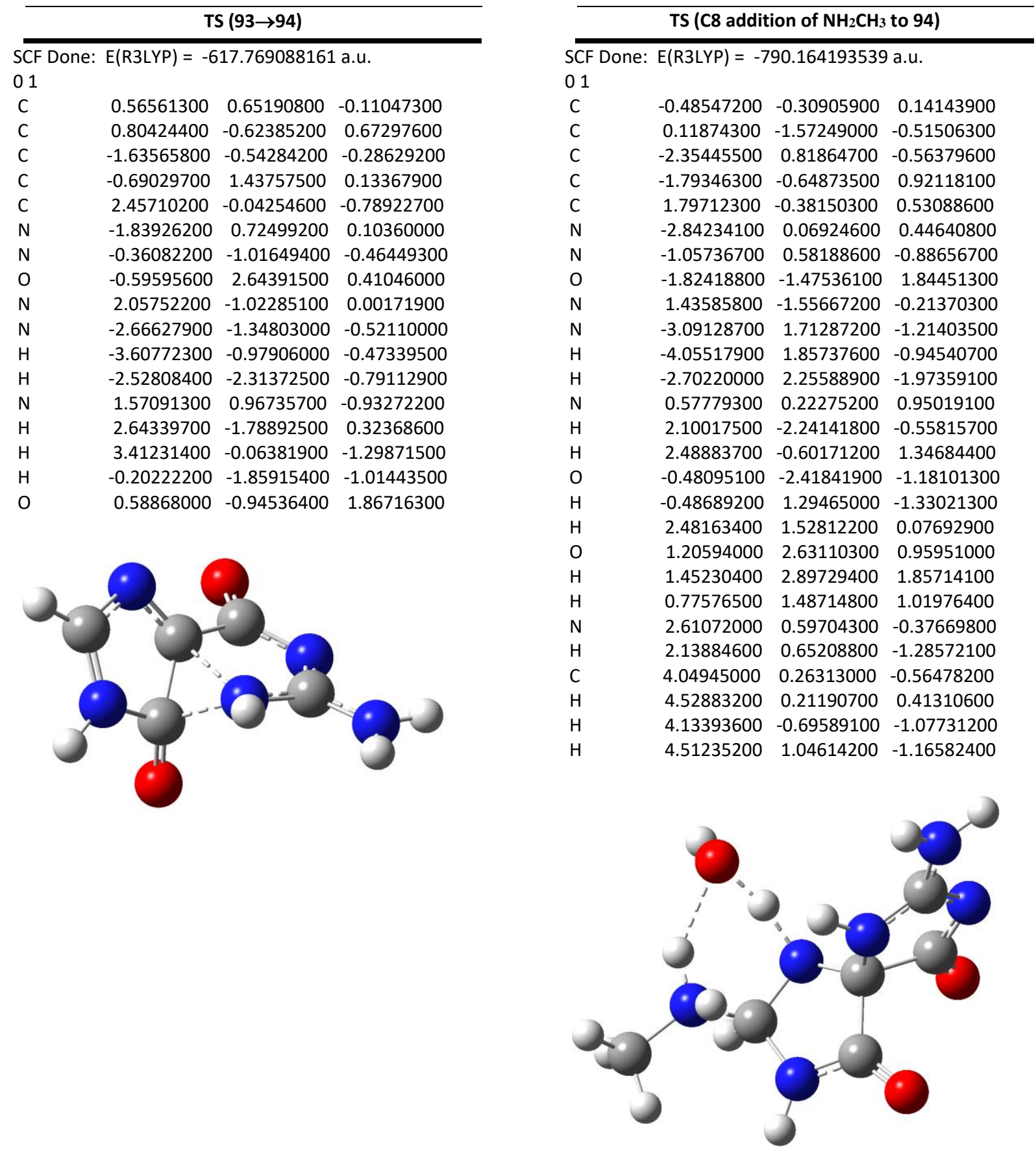


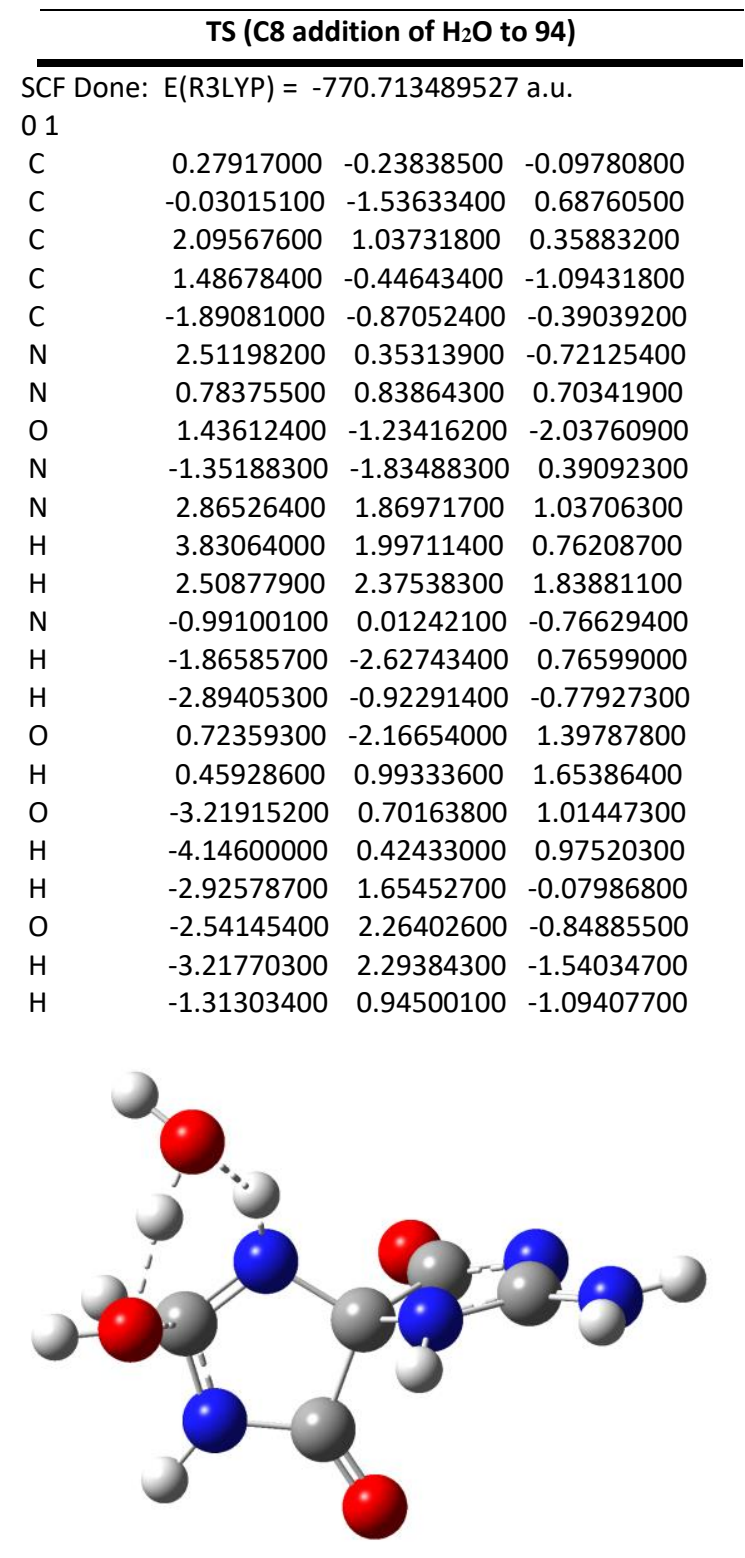

